Supporting information for:

\title{
Synthesis, Structure and Hydrothiolation Activity of Rhodium Pyrazolylborate Complexes
}

\author{
Lauren R. Fraser, Jeffrey Bird, Qiming Wu, Changsheng Cao, Brian O. Patrick and \\ Jennifer A. Love* \\ Department of Chemistry, 2036 Main Mall, University of British Columbia, \\ Vancouver, British Columbia V6T 1Z1, Canada
}

\section{Table of Contents}

NMR spectra of $\mathrm{Tp}^{\mathrm{Ph}} \mathrm{Rh}\left(\mathrm{PPh}_{3}\right)_{2} 4$

NMR spectra of $\mathrm{Tp}^{\mathrm{Ph}, \mathrm{Me}} \mathrm{Rh}\left(\mathrm{PPh}_{3}\right)_{2} \mathbf{5}$

NMR spectra of compound $15 \mathbf{a}$

NMR spectra of compound 15c

NMR spectra of compound 17a

NMR spectra of compound $18 a$

X-ray structure of $\mathrm{Bp}{ }^{*} \mathrm{Rh}\left(\mathrm{PPh}_{3}\right)_{2} 2$

X-ray report for 2

X-ray structure of $\mathrm{TpRh}\left(\mathrm{PPh}_{3}\right)_{2} 3$

X-ray report for 3

X-ray structure of $\mathrm{Tp}^{\mathrm{Ph}} \mathrm{Rh}\left(\mathrm{PPh}_{3}\right)_{2} 4$

X-ray report for 4

X-ray structure of $\mathrm{Tp}^{\mathrm{Ph}, \mathrm{Me}} \mathrm{Rh}\left(\mathrm{PPh}_{3}\right)_{2} 5$

X-ray report for 5

\section{Page}

S2

S3, S4

S5

S6

S7

S8

S9, S10

S11 - S31

S32, S33

S34 - S55

S56, S57

$\mathrm{S} 58-\mathrm{S} 80$

S81, S82

S83 - S104 


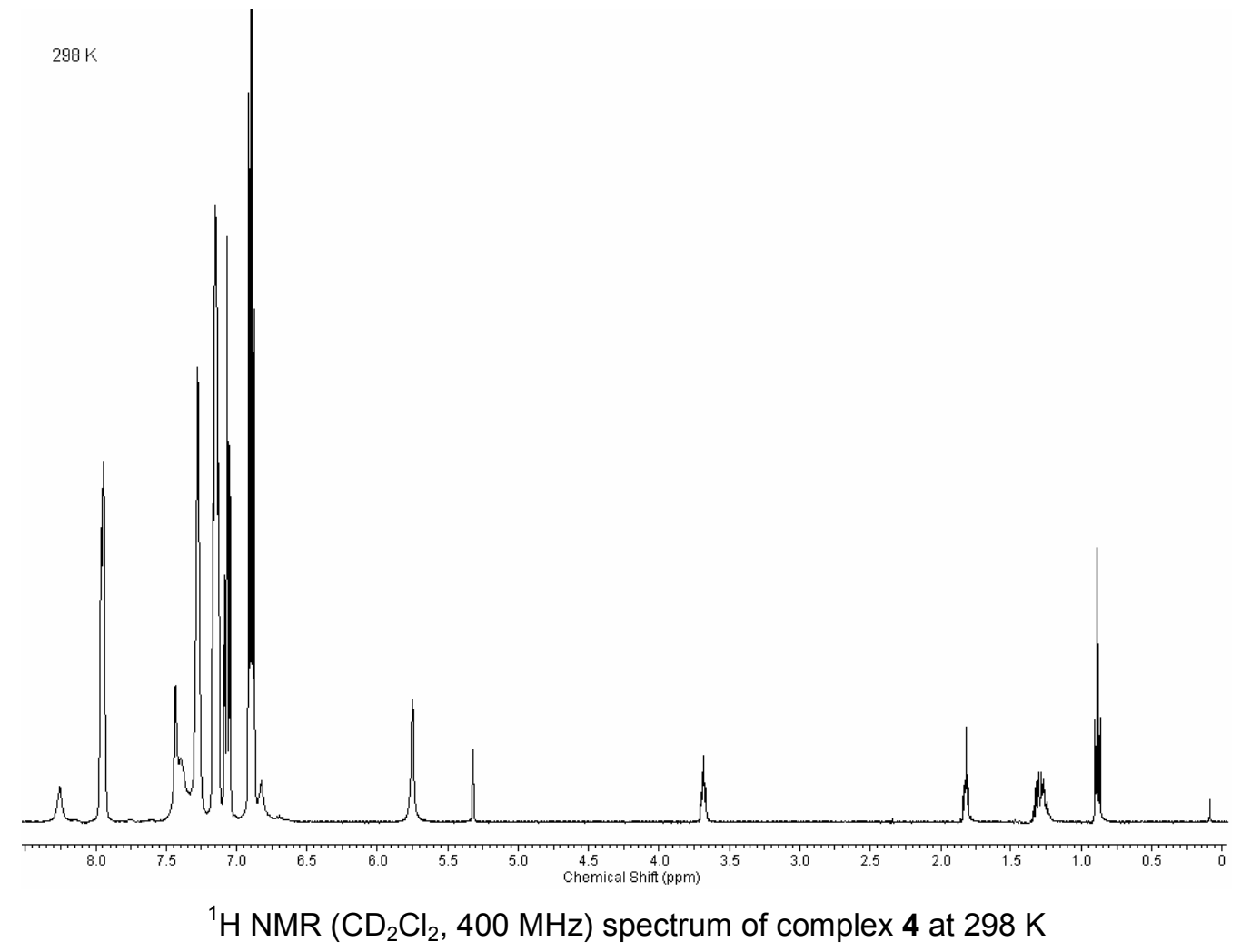

$298 K$

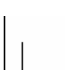

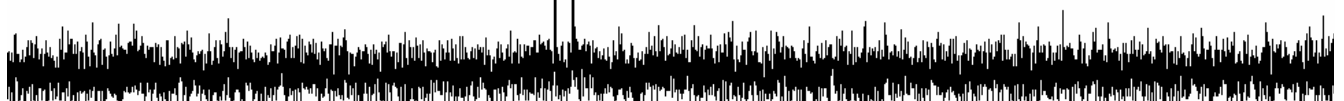
Nin

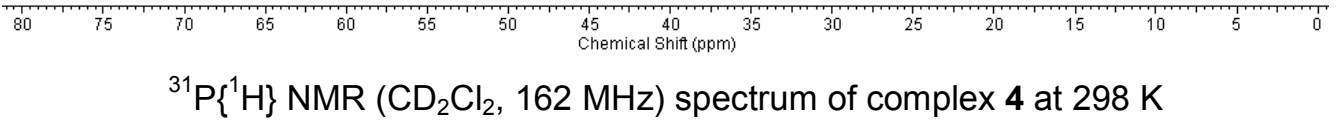



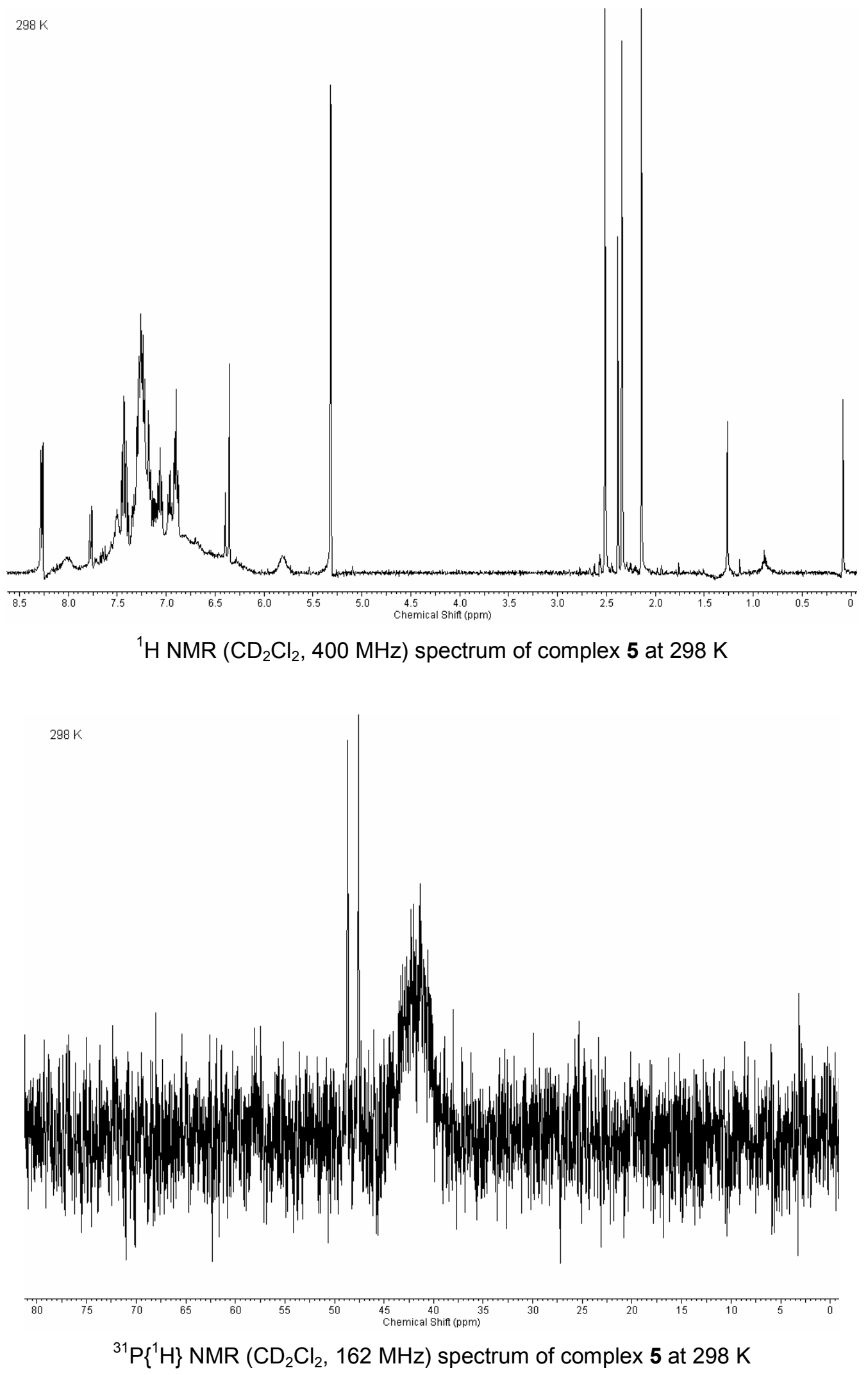


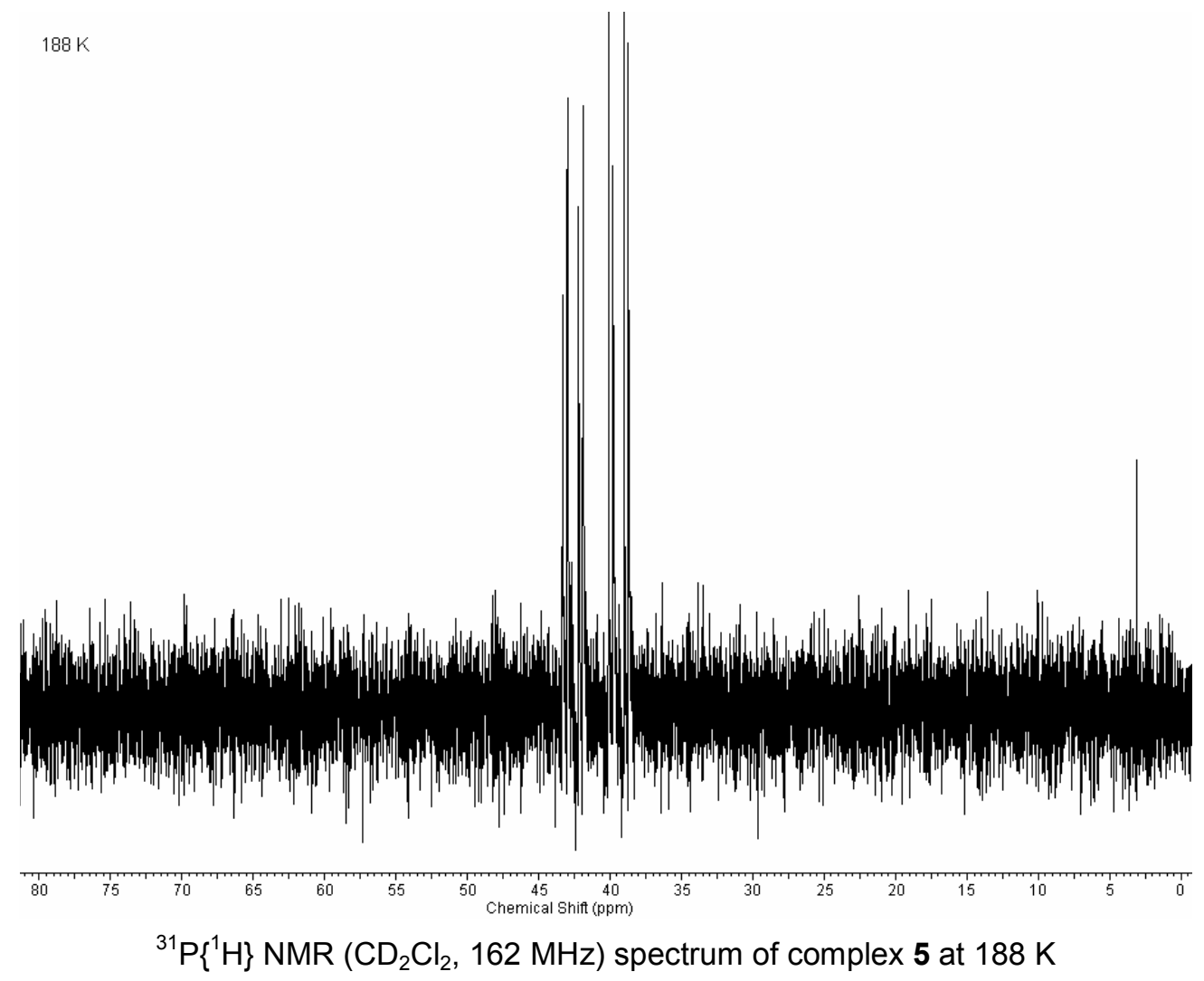




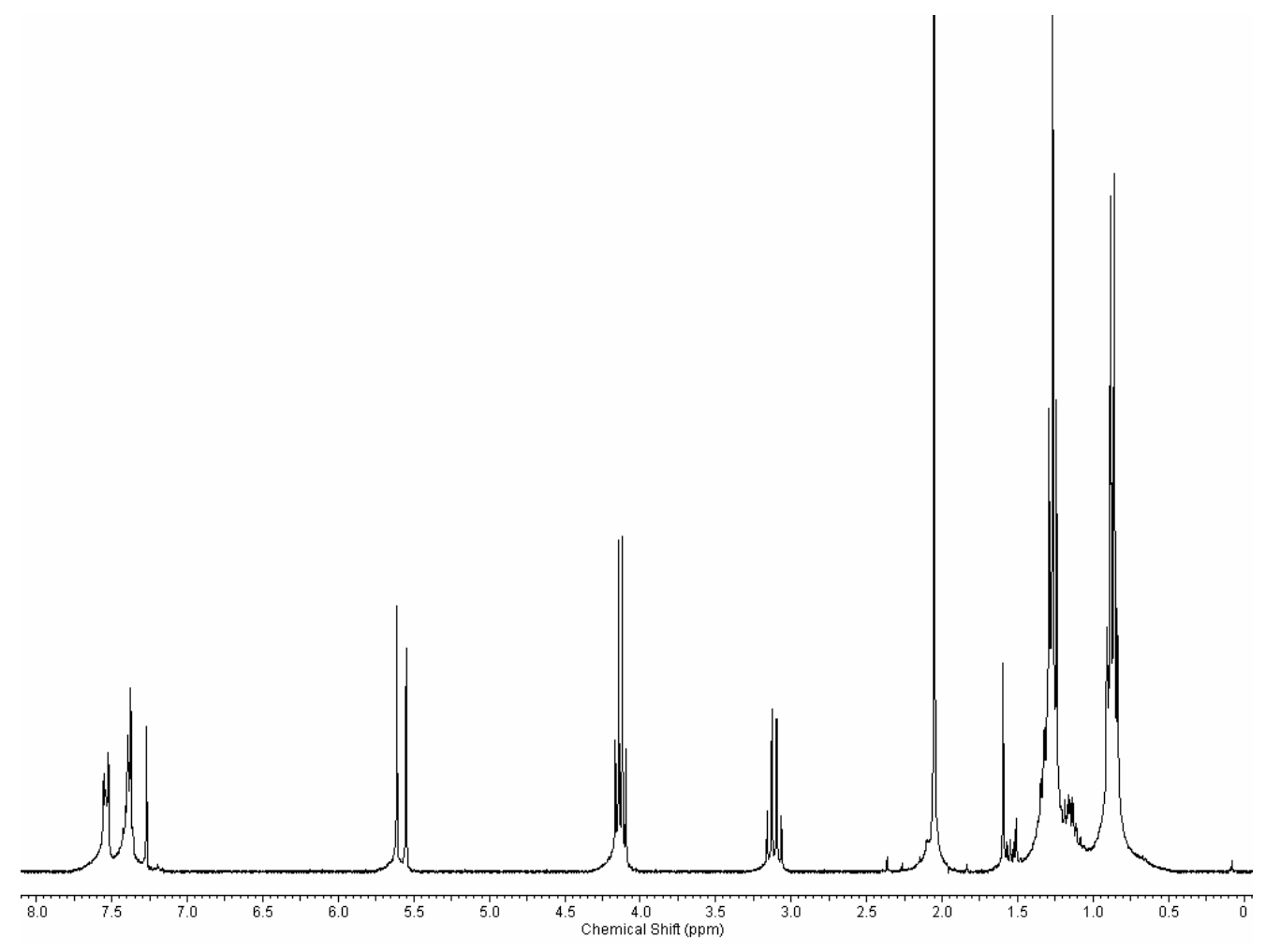

${ }^{1} \mathrm{H}$ NMR $\left(\mathrm{CDCl}_{3}, 300 \mathrm{MHz}\right)$ spectrum of compound 15a at $298 \mathrm{~K}$

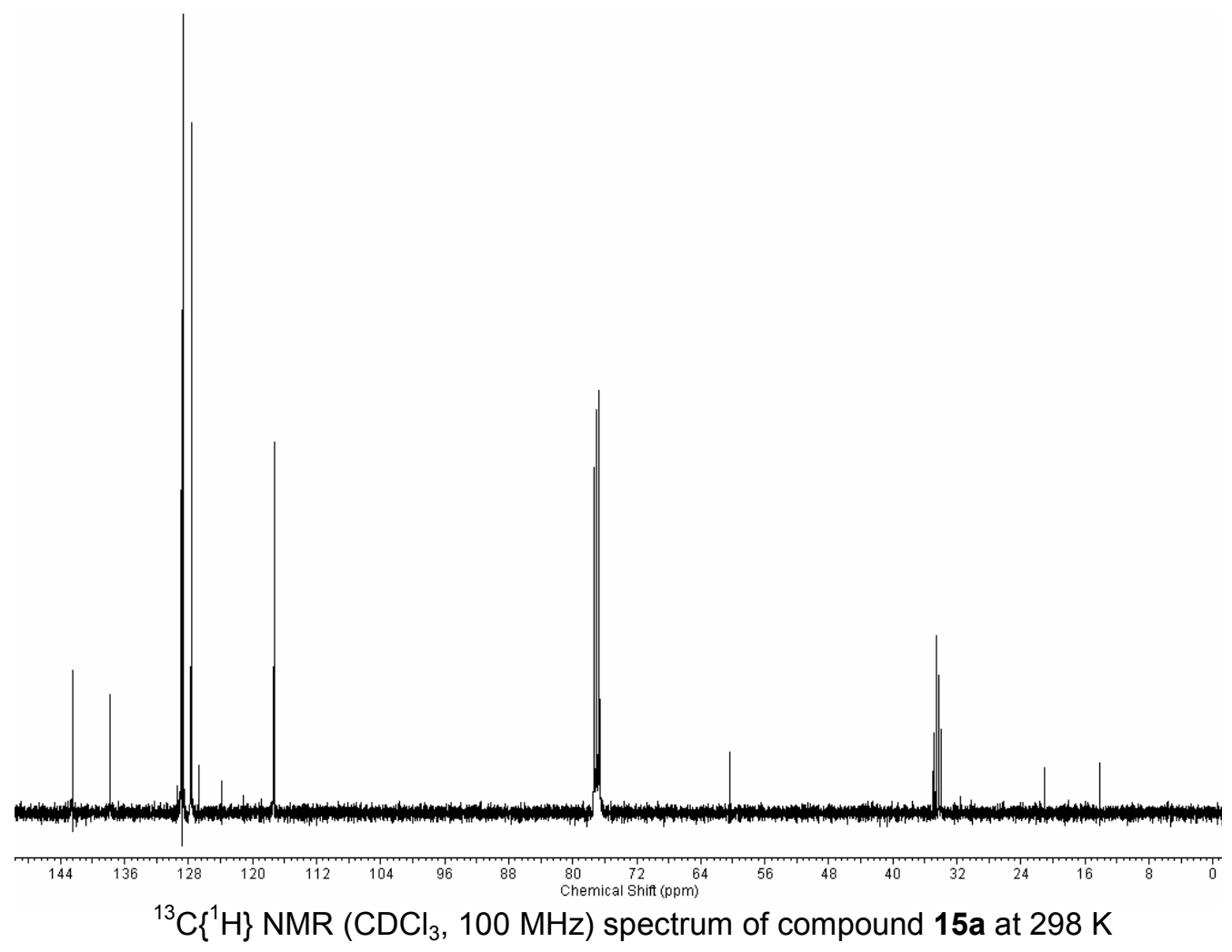


Compound 15c observed in situ as mixture with the $E$-linear isomer.
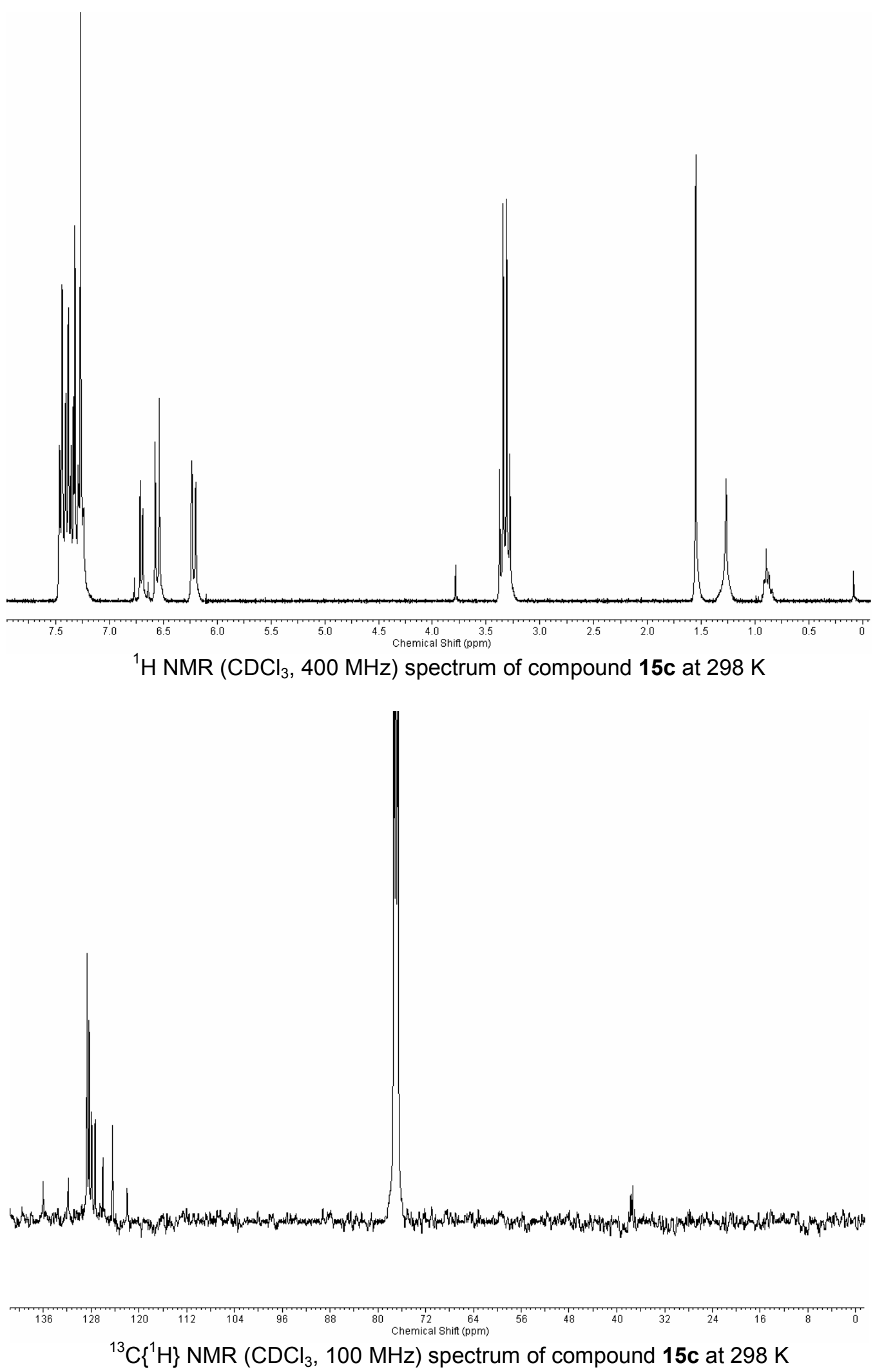

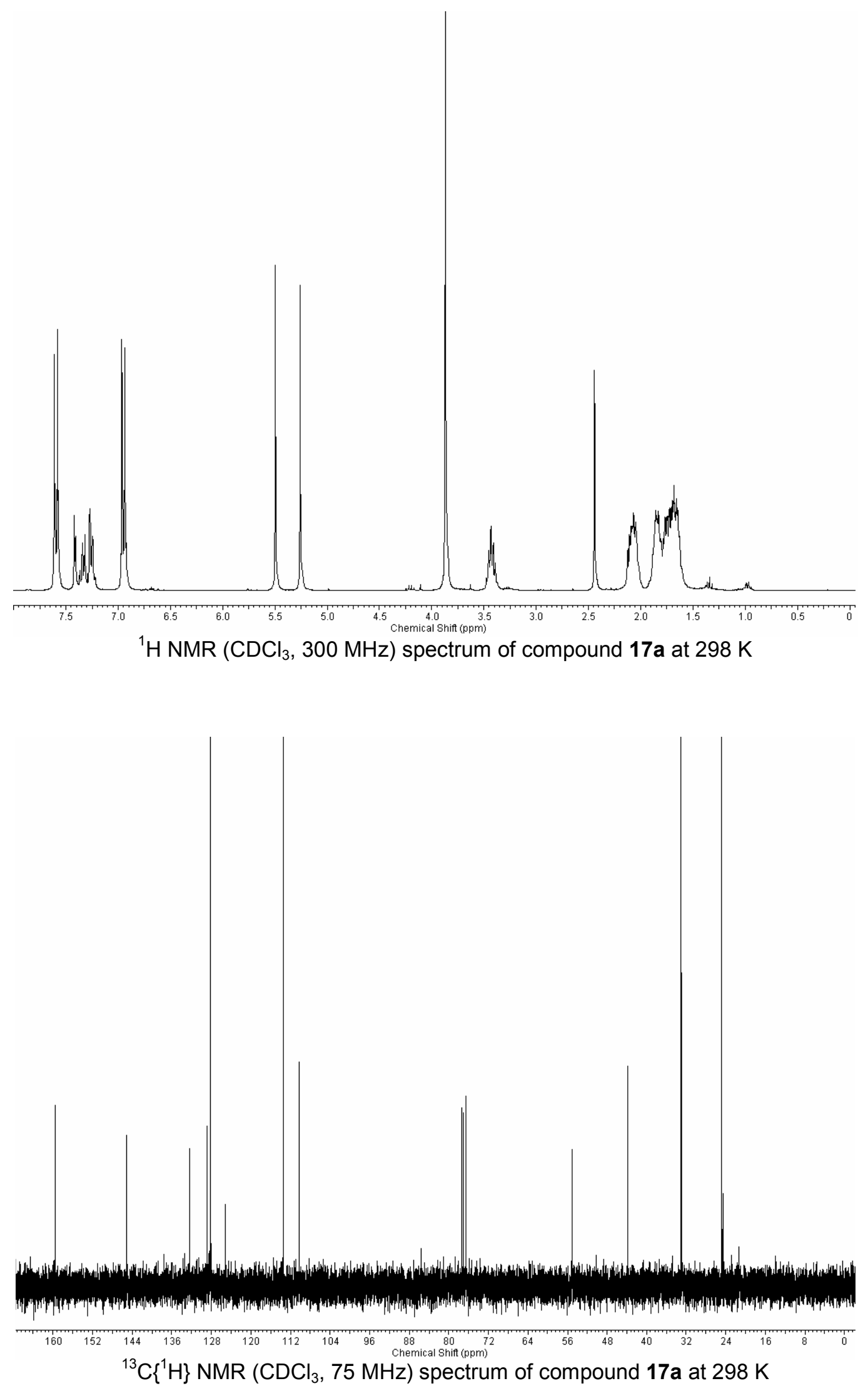

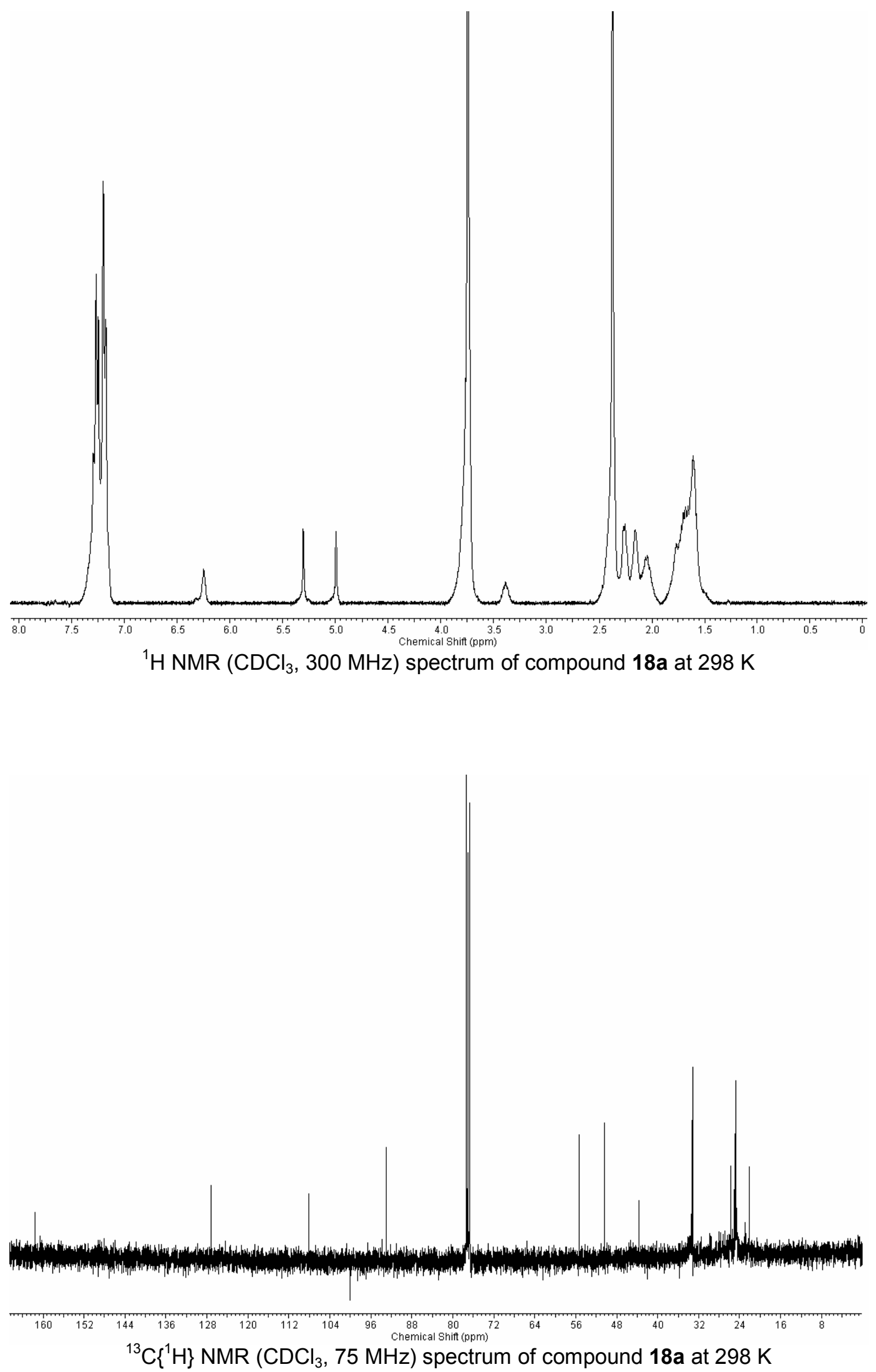
Figure S1. Crystal Structure of Complex 2 - Configuration represented in paper.

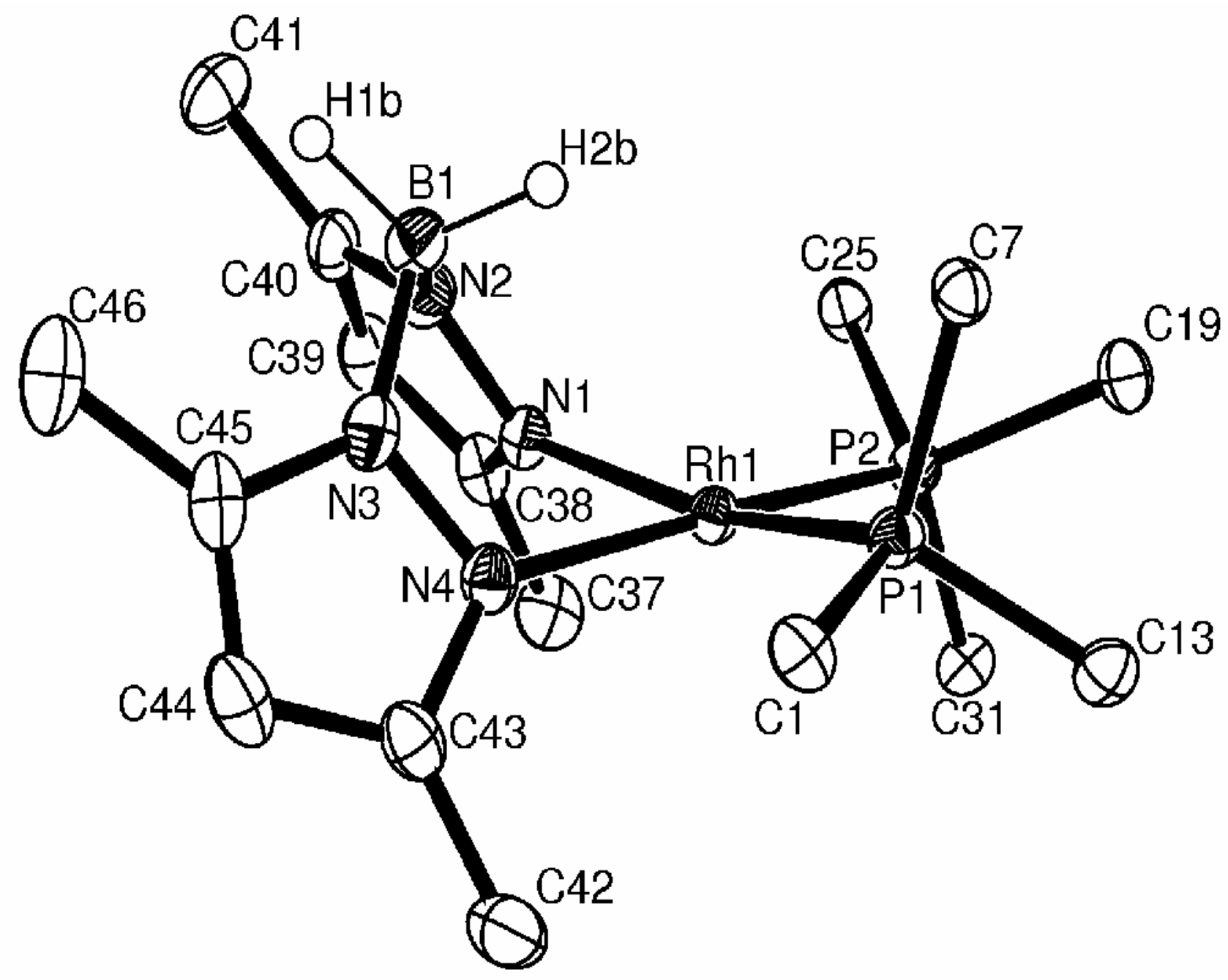


Figure S2. Crystal Structure of Complex 2 - Including all atoms.

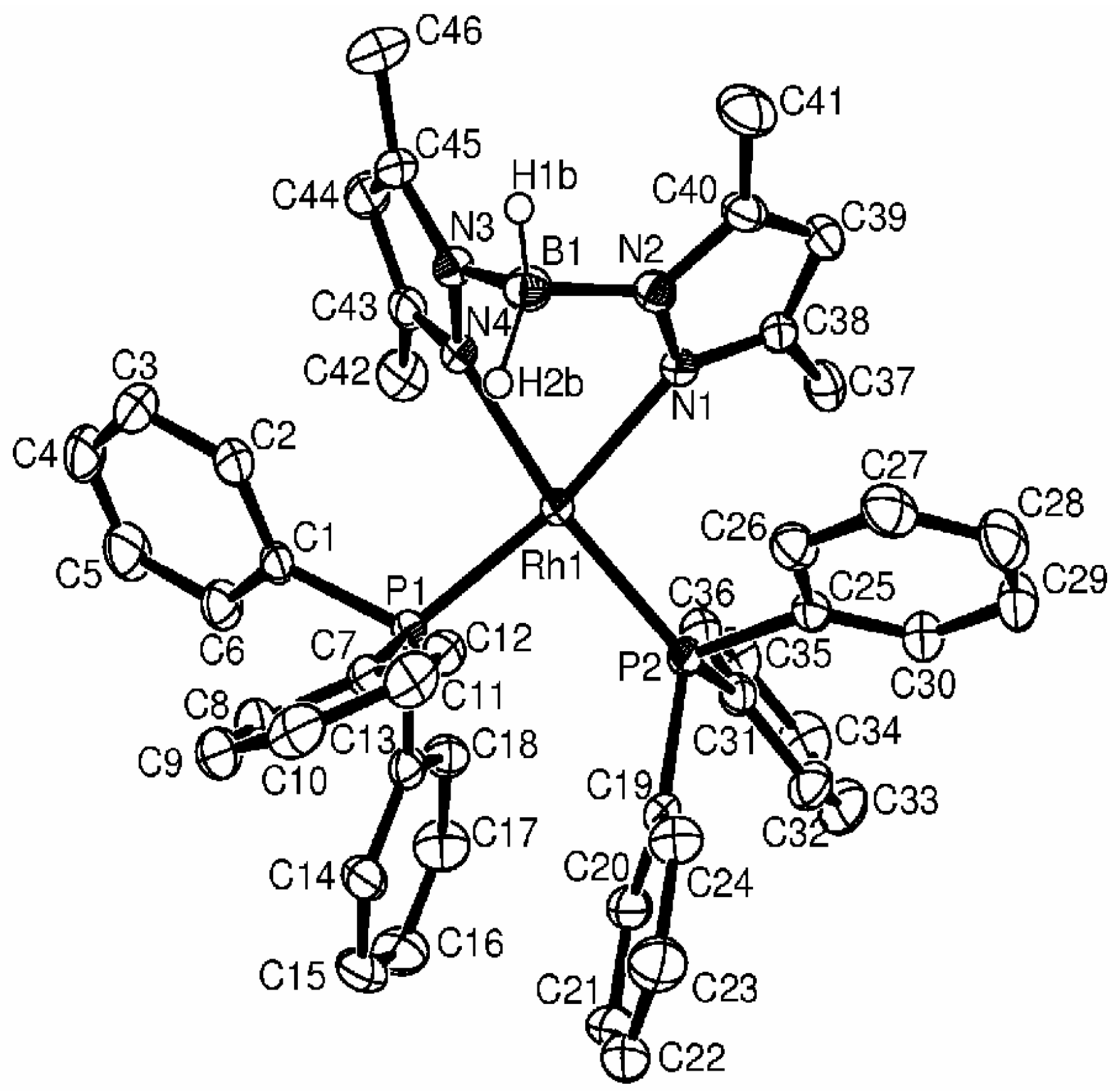




\section{Experimental}

\section{Data Collection}

An orange prism crystal of $\mathrm{C}_{46} \mathrm{H}_{46} \mathrm{BN}_{4} \mathrm{P}_{2} \mathrm{Rh} .1 / 2 \mathrm{C}_{6} \mathrm{H}_{14} \cdot{ }^{1} / 2 \mathrm{C}_{7} \mathrm{H}_{8}$ having approximate dimensions of $0.25 \mathrm{x}$ $0.25 \times 0.40 \mathrm{~mm}$ was mounted on a glass fiber. All measurements were made on a Bruker X8 APEX II diffractometer with graphite monochromated Mo-Ka radiation.

The data were collected at a temperature of $-100.0 \pm 0.1^{\circ} \mathrm{C}$ to a maximum $2 \theta$ value of $56.4^{\circ}$. Data were collected in a series of $\phi$ and $\omega$ scans in $0.50^{\circ}$ oscillations with 10.0 second exposures. The crystal-todetector distance was $36.00 \mathrm{~mm}$.

\section{Data Reduction}

Of the 50326 reflections that were collected, 11268 were unique $\left(R_{\mathrm{int}}=0.052\right)$; equivalent reflections were merged. Data were collected and integrated using the Bruker SAINT ${ }^{1}$ software package. The linear absorption coefficient, $\mu$, for Mo-K $\alpha$ radiation is $4.74 \mathrm{~cm}^{-1}$. Data were corrected for absorption effects using the multi-scan technique (SADABS ${ }^{2}$ ), with minimum and maximum transmission coefficients of 0.802 and 0.888 , respectively. The data were corrected for Lorentz and polarization effects.

\section{$\underline{\text { Structure Solution and Refinement }}$}

The structure was solved by direct methods ${ }^{3}$. The material crystallizes with both toluene and hexane in the lattice. In this case there is a 50:50 mixture of toluene and hexane occupying the same space in the asymmetric unit. Mild restraints were employed to maintain reasonable geometries for both solvent molecules. All non-hydrogen atoms were refined anisotropically. All B-H hydrogens were located in difference maps and refined isotropically. All other hydrogen atoms were placed in calculated positions and were not refined. The final cycle of full-matrix least-squares refinement ${ }^{4}$ on $\mathrm{F}^{2}$ was based on 11268 reflections and 573 variable parameters and converged (largest parameter shift was 0.00 times its esd) with unweighted and weighted agreement factors of:

$$
\mathrm{R} 1=\Sigma \| \mathrm{Fo}|-| \mathrm{Fc}|| / \Sigma|\mathrm{Fo}|=0.061
$$




$$
w R 2=\left[\Sigma\left(w\left(F o^{2}-F c^{2}\right)^{2}\right) / \Sigma w\left(F o^{2}\right)^{2}\right]^{1 / 2}=0.098
$$

The standard deviation of an observation of unit weight ${ }^{5}$ was 1.02 . The weighting scheme was based on counting statistics. The maximum and minimum peaks on the final difference Fourier map corresponded to 0.47 and $-0.44 \mathrm{e}^{-} / \AA^{3}$, respectively.

Neutral atom scattering factors were taken from Cromer and Waber 6 . Anomalous dispersion effects were included in $\mathrm{Fcalc}^{7}$; the values for $\Delta \mathrm{f}^{\prime}$ and $\Delta \mathrm{f}^{\prime \prime}$ were those of Creagh and McAuley ${ }^{8}$. The values for the mass attenuation coefficients are those of Creagh and Hubbell ${ }^{9}$. All refinements were performed using the SHELXTL 10 crystallographic software package of Bruker-AXS.

\section{References}

(1) SAINT. Version 7.03A. Bruker AXS Inc., Madison, Wisconsin, USA. (1997-2003).

(2) SADABS. Bruker Nonius area detector scaling and absorption correction - V2.10, Bruker AXS Inc., Madison, Wisconsin, USA (2003).

(3) SIR97 - Altomare A., Burla M.C., Camalli M., Cascarano G.L., Giacovazzo C. , Guagliardi A., Moliterni A.G.G., Polidori G.,Spagna R. (1999) J. Appl. Cryst. 32, 115-119.

(4) Least Squares function minimized:

$$
\Sigma w\left(\mathrm{Fo}_{\mathrm{o}}^{2}-\mathrm{F}_{\mathrm{C}}\right)^{2}
$$

(5) Standard deviation of an observation of unit weight:

$$
\begin{aligned}
& {\left[\Sigma w\left(\mathrm{~F}_{\mathrm{O}}{ }^{2}-\mathrm{F}_{\mathrm{C}}{ }^{2}\right)^{2} /\left(\mathrm{N}_{\mathrm{O}}-\mathrm{N}_{\mathrm{V}}\right)\right]^{1 / 2}} \\
& \text { where: } \mathrm{N}_{\mathrm{O}}=\text { number of observations } \\
& \qquad \mathrm{N}_{\mathrm{V}}=\text { number of variables }
\end{aligned}
$$

(6) Cromer, D. T. \& Waber, J. T.; "International Tables for X-ray Crystallography", Vol. IV, The Kynoch Press, Birmingham, England, Table 2.2 A (1974).

(7) Ibers, J. A. \& Hamilton, W. C.; Acta Crystallogr., 17, 781 (1964).

(8) Creagh, D. C. \& McAuley, W.J .; "International Tables for Crystallography", Vol C, (A.J.C. Wilson, ed.), Kluwer Academic Publishers, Boston, Table 4.2.6.8, pages 219-222 (1992).

(9) Creagh, D. C. \& Hubbell, J.H..; "International Tables for Crystallography", Vol C, (A.J.C. Wilson, ed.), Kluwer Academic Publishers, Boston, Table 4.2.4.3, pages 200-206 (1992).

(10) SHELXTL Version 5.1. Bruker AXS Inc., Madision, Wisconsin, USA. (1997). 
Table S1. Crystal Data and Structure Analysis Details for Complex 2.

A. Crystal Data

\author{
Empirical Formula \\ Formula Weight \\ Crystal Color, Habit \\ Crystal Dimensions \\ Crystal System \\ Lattice Type \\ Lattice Parameters
}

Space Group

$Z$ value

$D_{\text {calc }}$

F000

$\mu(\operatorname{MoK} \alpha)$
$\mathrm{C}_{52.5} \mathrm{H}_{57} \mathrm{BN}_{4} \mathrm{P}_{2} \mathrm{Rh}$

919.68

orange, prism

$0.25 \times 0.25 \times 0.40 \mathrm{~mm}$

monoclinic

primitive

$a=11.168(1) \AA$

$\mathrm{b}=17.004(2) \AA$

$\mathrm{c}=24.853(2) \AA$

$\alpha=90.0^{\circ}$

$\beta=98.470(6)^{\circ}$

$\gamma=90.0^{\circ}$

$\mathrm{V}=4667.9(8) \AA^{3}$

$P 2{ }_{1} / c(\# 14)$

4

$1.309 \mathrm{~g} / \mathrm{cm}^{3}$

1920.00

$4.74 \mathrm{~cm}^{-1}$ 
B. Intensity Measurements

Diffractometer

Radiation

Data Images

Detector Position

$2 \theta \max$

No. of Reflections Measured

Corrections
Bruker X8 APEX II

$\operatorname{MoK} \alpha(\lambda=0.71073 \AA)$

graphite monochromated

1322 exposures@10.0 seconds

$36.00 \mathrm{~mm}$

$56.4^{\circ}$

Total: 50326

Unique: $11268\left(\mathrm{R}_{\text {int }}=0.052\right)$

Absorption $\left(T_{\text {min }}=0.802, T_{\text {max }}=0.888\right.$ ) Lorentz-polarization 
C. Structure Solution and Refinement

\begin{tabular}{|c|c|}
\hline Structure Solution & Direct Methods (SIR97) \\
\hline Refinement & Full-matrix least-squares on $\mathrm{F}^{2}$ \\
\hline Function Minimized & $\Sigma w\left(F o^{2}-F c^{2}\right)^{2}$ \\
\hline Least Squares Weights & $w=1 /\left(\sigma^{2}\left(F o^{2}\right)+(0.0403 P)^{2}+3.468 P\right)$ \\
\hline Anomalous Dispersion & All non-hydrogen atoms \\
\hline No. Observations $(I>0.00 \sigma(I))$ & 11268 \\
\hline No. Variables & 573 \\
\hline Reflection/Parameter Ratio & 19.66 \\
\hline Residuals (refined on $\mathrm{F}^{2}$, all data): R1; wR2 & $0.061 ; 0.098$ \\
\hline Goodness of Fit Indicator & 1.02 \\
\hline No. Observations $(I>2.00 \sigma(I))$ & 8528 \\
\hline Residuals (refined on F): R1; wR2 & $0.039 ; 0.087$ \\
\hline Max Shift/Error in Final Cycle & 0.00 \\
\hline Maximum peak in Final Diff. Map & $0.47 \mathrm{e}^{-} / \AA^{3}$ \\
\hline Minimum peak in Final Diff. Map & $-0.44 e^{-} / \AA^{3}$ \\
\hline
\end{tabular}




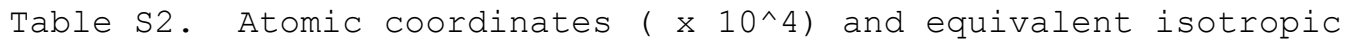
displacement parameters $\left(A^{\wedge} 2 \times 10^{\wedge} 3\right)$ for complex 2 .

U(eq) is defined as one third of the trace of the orthogonalized Uij tensor.

\begin{tabular}{|c|c|c|c|c|}
\hline & $x$ & $\mathrm{y}$ & $\mathrm{z}$ & $\mathrm{U}(\mathrm{eq})$ \\
\hline$C(1)$ & $-1026(2)$ & $1740(2)$ & $2262(1)$ & $22(1)$ \\
\hline$C(2)$ & $-1789(2)$ & $1090(2)$ & $2208(1)$ & $27(1)$ \\
\hline$C(3)$ & $-2540(3)$ & $947(2)$ & $1724(1)$ & $38(1)$ \\
\hline$C(4)$ & $-2549(3)$ & $1455(2)$ & $1294(1)$ & $44(1)$ \\
\hline$C(5)$ & $-1791(3)$ & $2100(2)$ & $1341(1)$ & $43(1)$ \\
\hline$C(6)$ & $-1036(3)$ & $2243(2)$ & $1824(1)$ & $30(1)$ \\
\hline$C(7)$ & $801(2)$ & $965(1)$ & $2956(1)$ & $20(1)$ \\
\hline$C(8)$ & $1185(2)$ & $627(2)$ & $2502(1)$ & $27(1)$ \\
\hline$C(9)$ & $1772(3)$ & $-93(2)$ & $2539(1)$ & $31(1)$ \\
\hline$C(10)$ & $1964(3)$ & $-495(2)$ & $3027(1)$ & $32(1)$ \\
\hline$C(11)$ & $1578(3)$ & $-174(2)$ & $3477(1)$ & $30(1)$ \\
\hline $\mathrm{C}(12)$ & 1001 (2) & $552(2)$ & $3443(1)$ & $25(1)$ \\
\hline C (13) & $960(2)$ & $2642(2)$ & $2732(1)$ & $22(1)$ \\
\hline$C(14)$ & $2059(2)$ & $2503(2)$ & $2554(1)$ & $29(1)$ \\
\hline$C(15)$ & $2723(3)$ & $3123(2)$ & $2384(1)$ & $38(1)$ \\
\hline$C(16)$ & $2286(3)$ & $3886(2)$ & $2389(1)$ & 41 (1) \\
\hline$C(17)$ & $1197(3)$ & $4029(2)$ & $2566(1)$ & $39(1)$ \\
\hline C (18) & $549(2)$ & $3414(2)$ & $2738(1)$ & $28(1)$ \\
\hline C (19) & $2169(2)$ & $2394(2)$ & $4100(1)$ & $23(1)$ \\
\hline$C(20)$ & $2801(2)$ & $2930(2)$ & $3824(1)$ & $30(1)$ \\
\hline$C(21)$ & $3998(3)$ & $2795(2)$ & $3767(1)$ & $37(1)$ \\
\hline$C(22)$ & $4590(3)$ & $2132(2)$ & $3982(1)$ & $38(1)$ \\
\hline$C(23)$ & $3977(3)$ & $1597(2)$ & $4248(1)$ & $40(1)$ \\
\hline$C(24)$ & $2781(3)$ & $1723(2)$ & $4310(1)$ & $31(1)$ \\
\hline$C(25)$ & $621(2)$ & $2186(2)$ & $4883(1)$ & $22(1)$ \\
\hline$C(26)$ & $374(2)$ & $1389(2)$ & $4946(1)$ & $27(1)$ \\
\hline$C(27)$ & $450(3)$ & $1051(2)$ & $5454(1)$ & $35(1)$ \\
\hline $\mathrm{C}(28)$ & $761(3)$ & $1504(2)$ & $5911(1)$ & $40(1)$ \\
\hline$C(29)$ & $983(3)$ & $2294(2)$ & $5859(1)$ & $38(1)$ \\
\hline$C(30)$ & $921(2)$ & $2637(2)$ & $5348(1)$ & $29(1)$ \\
\hline$C(31)$ & $542(2)$ & $3628(1)$ & $4235(1)$ & $22(1)$ \\
\hline$C(32)$ & $1478(3)$ & $4068(2)$ & $4529(1)$ & $32(1)$ \\
\hline$C(33)$ & $1413(3)$ & $4878(2)$ & $4552(1)$ & $41(1)$ \\
\hline$C(34)$ & $426(3)$ & $5265(2)$ & $4283(1)$ & $42(1)$ \\
\hline$C(35)$ & $-495(3)$ & $4851(2)$ & $3986(1)$ & $37(1)$ \\
\hline$C(36)$ & $-441(3)$ & $4034(2)$ & $3960(1)$ & $27(1)$ \\
\hline$C(37)$ & $-2211(3)$ & $3109(2)$ & $4792(1)$ & $31(1)$ \\
\hline$C(38)$ & $-2354(2)$ & $2244(2)$ & $4718(1)$ & $24(1)$ \\
\hline C (39) & $-2866(2)$ & $1713(2)$ & $5044(1)$ & $29(1)$ \\
\hline$C(40)$ & $-2860(2)$ & $996(2)$ & $4789(1)$ & $27(1)$ \\
\hline$C(41)$ & $-3306(3)$ & $213(2)$ & $4952(1)$ & $38(1)$ \\
\hline$C(42)$ & $-3374(3)$ & $2809(2)$ & $2614(1)$ & $36(1)$ \\
\hline$C(43)$ & $-3543(2)$ & $2013(2)$ & $2846(1)$ & $26(1)$ \\
\hline$C(44)$ & $-4529(3)$ & $1510(2)$ & $2753(1)$ & $34(1)$ \\
\hline$C(45)$ & $-4236(2)$ & $861(2)$ & $3073(1)$ & $30(1)$ \\
\hline$C(46)$ & $-4974(3)$ & $151(2)$ & $3159(1)$ & $49(1)$ \\
\hline$C(47)$ & $4466(8)$ & $1709(3)$ & $5879(3)$ & $56(2)$ \\
\hline
\end{tabular}




\begin{tabular}{|c|c|c|c|c|}
\hline$C(48)$ & $4332(6)$ & $2566(3)$ & $5700(3)$ & $43(2)$ \\
\hline$C(49)$ & $4193(6)$ & $3102(3)$ & $6176(2)$ & $33(1)$ \\
\hline$C(50)$ & $4054(5)$ & $3953(3)$ & $5998(2)$ & 32 (1) \\
\hline C ( 51) & $3959(6)$ & $4508(3)$ & $6472(2)$ & $44(2)$ \\
\hline C ( 52$)$ & $3938(7)$ & $5357(3)$ & $6300(4)$ & $65(2)$ \\
\hline B (1) & $-2368(3)$ & $532(2)$ & $3832(1)$ & $26(1)$ \\
\hline $\mathrm{N}(1)$ & $-2028(2)$ & $1869(1)$ & $4291(1)$ & $20(1)$ \\
\hline $\mathrm{N}(2)$ & $-2365(2)$ & $1091(1)$ & $4332(1)$ & $23(1)$ \\
\hline $\mathrm{N}(3)$ & $-3110(2)$ & $971(1)$ & $3349(1)$ & $24(1)$ \\
\hline $\mathrm{N}(4)$ & $-2684(2)$ & $1684(1)$ & $3204(1)$ & $21(1)$ \\
\hline $\mathrm{P}(1)$ & $-63(1)$ & $1891(1)$ & $2923(1)$ & $18(1)$ \\
\hline$P(2)$ & $573(1)$ & $2551(1)$ & $4183(1)$ & $18(1)$ \\
\hline $\operatorname{Rh}(1)$ & $-1027(1)$ & $2053(1)$ & $3635(1)$ & $17(1)$ \\
\hline C ( 53$)$ & 4191 (11) & $3011(6)$ & $5773(5)$ & $155(7)$ \\
\hline C ( 54$)$ & $4522(9)$ & $3305(7)$ & $5294(4)$ & $143(5)$ \\
\hline$C(55)$ & $4638(9)$ & $4111(8)$ & $5226(4)$ & $176(7)$ \\
\hline$C(56)$ & $4424(11)$ & $4624(6)$ & $5637(6)$ & 209 (8) \\
\hline$C(57)$ & $4093(11)$ & $4330(8)$ & $6115(5)$ & $178(8)$ \\
\hline$C(58)$ & 3976 (11) & $3524(8)$ & $6184(4)$ & $201(10)$ \\
\hline C ( 59) & $4330(30)$ & $2154(8)$ & $5893(8)$ & $264(15)$ \\
\hline
\end{tabular}


Table S3. Bond lengths [A] and angles [deg] for complex 2.

\begin{tabular}{|c|c|}
\hline$C(1)-C(6)$ & $1.384(4)$ \\
\hline$C(1)-C(2)$ & $1.390(4)$ \\
\hline$C(1)-P(1)$ & $1.842(2)$ \\
\hline$C(2)-C(3)$ & $1.383(4)$ \\
\hline $\mathrm{C}(2)-\mathrm{H}(2)$ & 0.9500 \\
\hline$C(3)-C(4)$ & $1.374(5)$ \\
\hline $\mathrm{C}(3)-\mathrm{H}(3)$ & 0.9500 \\
\hline$C(4)-C(5)$ & $1.379(5)$ \\
\hline $\mathrm{C}(4)-\mathrm{H}(4)$ & 0.9500 \\
\hline$C(5)-C(6)$ & $1.383(4)$ \\
\hline C (5) -H (5) & 0.9500 \\
\hline$C(6)-H(6)$ & 0.9500 \\
\hline$C(7)-C(12)$ & $1.387(3)$ \\
\hline$C(7)-C(8)$ & $1.389(3)$ \\
\hline$C(7)-P(1)$ & $1.842(2)$ \\
\hline$C(8)-C(9)$ & $1.385(4)$ \\
\hline $\mathrm{C}(8)-\mathrm{H}(8)$ & 0.9500 \\
\hline$C(9)-C(10)$ & $1.382(4)$ \\
\hline $\mathrm{C}(9)-\mathrm{H}(9)$ & 0.9500 \\
\hline$C(10)-C(11)$ & $1.370(4)$ \\
\hline $\mathrm{C}(10)-\mathrm{H}(10)$ & 0.9500 \\
\hline $\mathrm{C}(11)-\mathrm{C}(12)$ & $1.389(4)$ \\
\hline $\mathrm{C}(11)-\mathrm{H}(11)$ & 0.9500 \\
\hline $\mathrm{C}(12)-\mathrm{H}(12)$ & 0.9500 \\
\hline C (13) - C (14) & $1.386(4)$ \\
\hline$C(13)-C(18)$ & $1.392(4)$ \\
\hline$C(13)-P(1)$ & $1.822(3)$ \\
\hline$C(14)-C(15)$ & $1.389(4)$ \\
\hline $\mathrm{C}(14)-\mathrm{H}(14)$ & 0.9500 \\
\hline$C(15)-C(16)$ & $1.388(4)$ \\
\hline C (15) - H (15) & 0.9500 \\
\hline$C(16)-C(17)$ & $1.374(4)$ \\
\hline $\mathrm{C}(16)-\mathrm{H}(16)$ & 0.9500 \\
\hline C (17) -C (18) & $1.376(4)$ \\
\hline $\mathrm{C}(17)-\mathrm{H}(17)$ & 0.9500 \\
\hline $\mathrm{C}(18)-\mathrm{H}(18)$ & 0.9500 \\
\hline$C(19)-C(24)$ & $1.391(4)$ \\
\hline$C(19)-C(20)$ & $1.394(4)$ \\
\hline $\mathrm{C}(19)-\mathrm{P}(2)$ & $1.844(3)$ \\
\hline$C(20)-C(21)$ & $1.384(4)$ \\
\hline $\mathrm{C}(20)-\mathrm{H}(20)$ & 0.9500 \\
\hline$C(21)-C(22)$ & $1.374(4)$ \\
\hline $\mathrm{C}(21)-\mathrm{H}(21)$ & 0.9500 \\
\hline$C(22)-C(23)$ & $1.368(4)$ \\
\hline $\mathrm{C}(22)-\mathrm{H}(22)$ & 0.9500 \\
\hline$C(23)-C(24)$ & $1.384(4)$ \\
\hline $\mathrm{C}(23)-\mathrm{H}(23)$ & 0.9500 \\
\hline $\mathrm{C}(24)-\mathrm{H}(24)$ & 0.9500 \\
\hline$C(25)-C(30)$ & $1.386(4)$ \\
\hline$C(25)-C(26)$ & $1.396(4)$ \\
\hline$C(25)-P(2)$ & $1.840(3)$ \\
\hline$C(26)-C(27)$ & $1.380(4)$ \\
\hline $\mathrm{C}(26)-\mathrm{H}(26)$ & 0.9500 \\
\hline C (27) - C (28) & $1.374(4)$ \\
\hline
\end{tabular}




\begin{tabular}{|c|c|}
\hline $\mathrm{C}(27)-\mathrm{H}(27)$ & 0.9500 \\
\hline$C(28)-C(29)$ & $1.376(5)$ \\
\hline $\mathrm{C}(28)-\mathrm{H}(28)$ & 0.9500 \\
\hline$C(29)-C(30)$ & $1.391(4)$ \\
\hline $\mathrm{C}(29)-\mathrm{H}(29)$ & 0.9500 \\
\hline $\mathrm{C}(30)-\mathrm{H}(30)$ & 0.9500 \\
\hline$C(31)-C(36)$ & $1.388(4)$ \\
\hline$C(31)-C(32)$ & $1.401(4)$ \\
\hline $\mathrm{C}(31)-\mathrm{P}(2)$ & $1.837(3)$ \\
\hline$C(32)-C(33)$ & $1.382(4)$ \\
\hline $\mathrm{C}(32)-\mathrm{H}(32)$ & 0.9500 \\
\hline$C(33)-C(34)$ & $1.370(5)$ \\
\hline $\mathrm{C}(33)-\mathrm{H}(33)$ & 0.9500 \\
\hline$C(34)-C(35)$ & $1.370(4)$ \\
\hline $\mathrm{C}(34)-\mathrm{H}(34)$ & 0.9500 \\
\hline$C(35)-C(36)$ & $1.393(4)$ \\
\hline $\mathrm{C}(35)-\mathrm{H}(35)$ & 0.9500 \\
\hline $\mathrm{C}(36)-\mathrm{H}(36)$ & 0.9500 \\
\hline$C(37)-C(38)$ & $1.487(4)$ \\
\hline $\mathrm{C}(37)-\mathrm{H}(37 \mathrm{~A})$ & 0.9800 \\
\hline $\mathrm{C}(37)-\mathrm{H}(37 \mathrm{~B})$ & 0.9800 \\
\hline $\mathrm{C}(37)-\mathrm{H}(37 \mathrm{C})$ & 0.9800 \\
\hline $\mathrm{C}(38)-\mathrm{N}(1)$ & $1.333(3)$ \\
\hline$C(38)-C(39)$ & $1.392(4)$ \\
\hline$C(39)-C(40)$ & $1.374(4)$ \\
\hline $\mathrm{C}(39)-\mathrm{H}(39)$ & 0.9500 \\
\hline $\mathrm{C}(40)-\mathrm{N}(2)$ & $1.342(3)$ \\
\hline$C(40)-C(41)$ & $1.498(4)$ \\
\hline $\mathrm{C}(41)-\mathrm{H}(41 \mathrm{~A})$ & 0.9800 \\
\hline $\mathrm{C}(41)-\mathrm{H}(41 \mathrm{~B})$ & 0.9800 \\
\hline $\mathrm{C}(41)-\mathrm{H}(41 \mathrm{C})$ & 0.9800 \\
\hline$C(42)-C(43)$ & $1.494(4)$ \\
\hline $\mathrm{C}(42)-\mathrm{H}(42 \mathrm{~A})$ & 0.9800 \\
\hline $\mathrm{C}(42)-\mathrm{H}(42 \mathrm{~B})$ & 0.9800 \\
\hline $\mathrm{C}(42)-\mathrm{H}(42 \mathrm{C})$ & 0.9800 \\
\hline$C(43)-N(4)$ & $1.332(3)$ \\
\hline$C(43)-C(44)$ & $1.387(4)$ \\
\hline$C(44)-C(45)$ & $1.373(4)$ \\
\hline $\mathrm{C}(44)-\mathrm{H}(44)$ & 0.9500 \\
\hline $\mathrm{C}(45)-\mathrm{N}(3)$ & $1.353(3)$ \\
\hline$C(45)-C(46)$ & $1.495(4)$ \\
\hline $\mathrm{C}(46)-\mathrm{H}(46 \mathrm{~A})$ & 0.9800 \\
\hline $\mathrm{C}(46)-\mathrm{H}(46 \mathrm{~B})$ & 0.9800 \\
\hline $\mathrm{C}(46)-\mathrm{H}(46 \mathrm{C})$ & 0.9800 \\
\hline$C(47)-C(48)$ & $1.526(7)$ \\
\hline $\mathrm{C}(47)-\mathrm{H}(47 \mathrm{~A})$ & 0.9800 \\
\hline $\mathrm{C}(47)-\mathrm{H}(47 \mathrm{~B})$ & 0.9800 \\
\hline $\mathrm{C}(47)-\mathrm{H}(47 \mathrm{C})$ & 0.9800 \\
\hline$C(48)-C(49)$ & $1.520(6)$ \\
\hline $\mathrm{C}(48)-\mathrm{H}(48 \mathrm{~A})$ & 0.9900 \\
\hline $\mathrm{C}(48)-\mathrm{H}(48 \mathrm{~B})$ & 0.9900 \\
\hline$C(49)-C(50)$ & $1.514(6)$ \\
\hline $\mathrm{C}(49)-\mathrm{H}(49 \mathrm{~A})$ & 0.9900 \\
\hline $\mathrm{C}(49)-\mathrm{H}(49 \mathrm{~B})$ & 0.9900 \\
\hline$C(50)-C(51)$ & $1.524(6)$ \\
\hline $\mathrm{C}(50)-\mathrm{H}(50 \mathrm{~A})$ & 0.9900 \\
\hline $\mathrm{C}(50)-\mathrm{H}(50 \mathrm{~B})$ & 0.9900 \\
\hline
\end{tabular}




\begin{tabular}{|c|c|}
\hline$C(51)-C(52)$ & $1.506(6)$ \\
\hline $\mathrm{C}(51)-\mathrm{H}(51 \mathrm{~A})$ & 0.9900 \\
\hline $\mathrm{C}(51)-\mathrm{H}(51 \mathrm{~B})$ & 0.9900 \\
\hline $\mathrm{C}(52)-\mathrm{H}(52 \mathrm{~A})$ & 0.9800 \\
\hline $\mathrm{C}(52)-\mathrm{H}(52 \mathrm{~B})$ & 0.9800 \\
\hline $\mathrm{C}(52)-\mathrm{H}(52 \mathrm{C})$ & 0.9800 \\
\hline $\mathrm{B}(1)-\mathrm{N}(3)$ & $1.547(4)$ \\
\hline $\mathrm{B}(1)-\mathrm{N}(2)$ & $1.564(4)$ \\
\hline $\mathrm{B}(1)-\mathrm{H}(1 \mathrm{~B})$ & $1.09(3)$ \\
\hline $\mathrm{B}(1)-\mathrm{H}(2 \mathrm{~B})$ & $1.16(3)$ \\
\hline$N(1)-N(2)$ & $1.384(3)$ \\
\hline $\mathrm{N}(1)-\mathrm{Rh}(1)$ & $2.132(2)$ \\
\hline$N(3)-N(4)$ & $1.369(3)$ \\
\hline $\mathrm{N}(4)-\mathrm{Rh}(1)$ & $2.092(2)$ \\
\hline $\mathrm{P}(1)-\mathrm{Rh}(1)$ & $2.2202(7)$ \\
\hline $\mathrm{P}(2)-\mathrm{Rh}(1)$ & $2.2468(7)$ \\
\hline$C(53)-C(54)$ & 1.3900 \\
\hline$C(53)-C(58)$ & 1.3900 \\
\hline$C(53)-C(59)$ & $1.490(9)$ \\
\hline$C(54)-C(55)$ & 1.3900 \\
\hline $\mathrm{C}(54)-\mathrm{H}(54)$ & 0.9500 \\
\hline$C(55)-C(56)$ & 1.3900 \\
\hline $\mathrm{C}(55)-\mathrm{H}(55)$ & 0.9500 \\
\hline$C(56)-C(57)$ & 1.3900 \\
\hline $\mathrm{C}(56)-\mathrm{H}(56)$ & 0.9500 \\
\hline$C(57)-C(58)$ & 1.3900 \\
\hline $\mathrm{C}(57)-\mathrm{H}(57)$ & 0.9500 \\
\hline $\mathrm{C}(58)-\mathrm{H}(58)$ & 0.9500 \\
\hline $\mathrm{C}(59)-\mathrm{H}(59 \mathrm{~A})$ & 0.9800 \\
\hline $\mathrm{C}(59)-\mathrm{H}(59 \mathrm{~B})$ & 0.9800 \\
\hline $\mathrm{C}(59)-\mathrm{H}(59 \mathrm{C})$ & 0.9800 \\
\hline$C(6)-C(1)-C(2)$ & $118.8(2)$ \\
\hline$C(6)-C(1)-P(1)$ & $123.5(2)$ \\
\hline$C(2)-C(1)-P(1)$ & $117.77(19)$ \\
\hline$C(3)-C(2)-C(1)$ & $120.6(3)$ \\
\hline $\mathrm{C}(3)-\mathrm{C}(2)-\mathrm{H}(2)$ & 119.7 \\
\hline $\mathrm{C}(1)-\mathrm{C}(2)-\mathrm{H}(2)$ & 119.7 \\
\hline$C(4)-C(3)-C(2)$ & $120.1(3)$ \\
\hline $\mathrm{C}(4)-\mathrm{C}(3)-\mathrm{H}(3)$ & 120.0 \\
\hline $\mathrm{C}(2)-\mathrm{C}(3)-\mathrm{H}(3)$ & 120.0 \\
\hline$C(3)-C(4)-C(5)$ & $120.0(3)$ \\
\hline $\mathrm{C}(3)-\mathrm{C}(4)-\mathrm{H}(4)$ & 120.0 \\
\hline $\mathrm{C}(5)-\mathrm{C}(4)-\mathrm{H}(4)$ & 120.0 \\
\hline$C(4)-C(5)-C(6)$ & $120.1(3)$ \\
\hline $\mathrm{C}(4)-\mathrm{C}(5)-\mathrm{H}(5)$ & 119.9 \\
\hline $\mathrm{C}(6)-\mathrm{C}(5)-\mathrm{H}(5)$ & 119.9 \\
\hline$C(5)-C(6)-C(1)$ & $120.6(3)$ \\
\hline$C(5)-C(6)-H(6)$ & 119.7 \\
\hline $\mathrm{C}(1)-\mathrm{C}(6)-\mathrm{H}(6)$ & 119.7 \\
\hline$C(12)-C(7)-C(8)$ & $118.0(2)$ \\
\hline$C(12)-C(7)-P(1)$ & $119.21(19)$ \\
\hline $\mathrm{C}(8)-\mathrm{C}(7)-\mathrm{P}(1)$ & $122.57(19)$ \\
\hline$C(9)-C(8)-C(7)$ & $120.7(3)$ \\
\hline $\mathrm{C}(9)-\mathrm{C}(8)-\mathrm{H}(8)$ & 119.6 \\
\hline $\mathrm{C}(7)-\mathrm{C}(8)-\mathrm{H}(8)$ & 119.6 \\
\hline$C(10)-C(9)-C(8)$ & $120.4(3)$ \\
\hline
\end{tabular}




\begin{tabular}{|c|c|}
\hline $\mathrm{C}(10)-\mathrm{C}(9)-\mathrm{H}(9)$ & 119.8 \\
\hline $\mathrm{C}(8)-\mathrm{C}(9)-\mathrm{H}(9)$ & 119.8 \\
\hline$C(11)-C(10)-C(9)$ & $119.5(3)$ \\
\hline $\mathrm{C}(11)-\mathrm{C}(10)-\mathrm{H}(10)$ & 120.3 \\
\hline$C(9)-C(10)-H(10)$ & 120.3 \\
\hline$C(10)-C(11)-C(12)$ & $120.2(3)$ \\
\hline $\mathrm{C}(10)-\mathrm{C}(11)-\mathrm{H}(11)$ & 119.9 \\
\hline $\mathrm{C}(12)-\mathrm{C}(11)-\mathrm{H}(11)$ & 119.9 \\
\hline$C(7)-C(12)-C(11)$ & $121.1(3)$ \\
\hline $\mathrm{C}(7)-\mathrm{C}(12)-\mathrm{H}(12)$ & 119.4 \\
\hline $\mathrm{C}(11)-\mathrm{C}(12)-\mathrm{H}(12)$ & 119.4 \\
\hline$C(14)-C(13)-C(18)$ & $118.3(2)$ \\
\hline$C(14)-C(13)-P(1)$ & $125.6(2)$ \\
\hline$C(18)-C(13)-P(1)$ & $116.0(2)$ \\
\hline$C(13)-C(14)-C(15)$ & $120.3(3)$ \\
\hline $\mathrm{C}(13)-\mathrm{C}(14)-\mathrm{H}(14)$ & 119.8 \\
\hline $\mathrm{C}(15)-\mathrm{C}(14)-\mathrm{H}(14)$ & 119.8 \\
\hline$C(16)-C(15)-C(14)$ & $120.2(3)$ \\
\hline $\mathrm{C}(16)-\mathrm{C}(15)-\mathrm{H}(15)$ & 119.9 \\
\hline $\mathrm{C}(14)-\mathrm{C}(15)-\mathrm{H}(15)$ & 119.9 \\
\hline$C(17)-C(16)-C(15)$ & $119.8(3)$ \\
\hline $\mathrm{C}(17)-\mathrm{C}(16)-\mathrm{H}(16)$ & 120.1 \\
\hline $\mathrm{C}(15)-\mathrm{C}(16)-\mathrm{H}(16)$ & 120.1 \\
\hline$C(16)-C(17)-C(18)$ & $119.8(3)$ \\
\hline $\mathrm{C}(16)-\mathrm{C}(17)-\mathrm{H}(17)$ & 120.1 \\
\hline $\mathrm{C}(18)-\mathrm{C}(17)-\mathrm{H}(17)$ & 120.1 \\
\hline$C(17)-C(18)-C(13)$ & $121.6(3)$ \\
\hline $\mathrm{C}(17)-\mathrm{C}(18)-\mathrm{H}(18)$ & 119.2 \\
\hline $\mathrm{C}(13)-\mathrm{C}(18)-\mathrm{H}(18)$ & 119.2 \\
\hline$C(24)-C(19)-C(20)$ & $117.7(2)$ \\
\hline$C(24)-C(19)-P(2)$ & $120.4(2)$ \\
\hline$C(20)-C(19)-P(2)$ & $121.9(2)$ \\
\hline$C(21)-C(20)-C(19)$ & $120.5(3)$ \\
\hline $\mathrm{C}(21)-\mathrm{C}(20)-\mathrm{H}(20)$ & 119.7 \\
\hline $\mathrm{C}(19)-\mathrm{C}(20)-\mathrm{H}(20)$ & 119.7 \\
\hline$C(22)-C(21)-C(20)$ & $121.0(3)$ \\
\hline $\mathrm{C}(22)-\mathrm{C}(21)-\mathrm{H}(21)$ & 119.5 \\
\hline $\mathrm{C}(20)-\mathrm{C}(21)-\mathrm{H}(21)$ & 119.5 \\
\hline$C(23)-C(22)-C(21)$ & $119.1(3)$ \\
\hline $\mathrm{C}(23)-\mathrm{C}(22)-\mathrm{H}(22)$ & 120.4 \\
\hline $\mathrm{C}(21)-\mathrm{C}(22)-\mathrm{H}(22)$ & 120.4 \\
\hline$C(22)-C(23)-C(24)$ & $120.8(3)$ \\
\hline $\mathrm{C}(22)-\mathrm{C}(23)-\mathrm{H}(23)$ & 119.6 \\
\hline $\mathrm{C}(24)-\mathrm{C}(23)-\mathrm{H}(23)$ & 119.6 \\
\hline$C(23)-C(24)-C(19)$ & $120.9(3)$ \\
\hline $\mathrm{C}(23)-\mathrm{C}(24)-\mathrm{H}(24)$ & 119.5 \\
\hline $\mathrm{C}(19)-\mathrm{C}(24)-\mathrm{H}(24)$ & 119.5 \\
\hline$C(30)-C(25)-C(26)$ & $118.1(2)$ \\
\hline$C(30)-C(25)-P(2)$ & $124.9(2)$ \\
\hline$C(26)-C(25)-P(2)$ & $116.96(19)$ \\
\hline$C(27)-C(26)-C(25)$ & $121.3(3)$ \\
\hline $\mathrm{C}(27)-\mathrm{C}(26)-\mathrm{H}(26)$ & 119.3 \\
\hline $\mathrm{C}(25)-\mathrm{C}(26)-\mathrm{H}(26)$ & 119.3 \\
\hline$C(28)-C(27)-C(26)$ & $119.9(3)$ \\
\hline $\mathrm{C}(28)-\mathrm{C}(27)-\mathrm{H}(27)$ & 120.1 \\
\hline $\mathrm{C}(26)-\mathrm{C}(27)-\mathrm{H}(27)$ & 120.1 \\
\hline$C(27)-C(28)-C(29)$ & $119.8(3)$ \\
\hline
\end{tabular}




\begin{tabular}{|c|c|}
\hline $\mathrm{C}(27)-\mathrm{C}(28)-\mathrm{H}(28)$ & 120.1 \\
\hline $\mathrm{C}(29)-\mathrm{C}(28)-\mathrm{H}(28)$ & 120.1 \\
\hline$C(28)-C(29)-C(30)$ & $120.7(3)$ \\
\hline $\mathrm{C}(28)-\mathrm{C}(29)-\mathrm{H}(29)$ & 119.7 \\
\hline $\mathrm{C}(30)-\mathrm{C}(29)-\mathrm{H}(29)$ & 119.7 \\
\hline$C(25)-C(30)-C(29)$ & $120.2(3)$ \\
\hline $\mathrm{C}(25)-\mathrm{C}(30)-\mathrm{H}(30)$ & 119.9 \\
\hline $\mathrm{C}(29)-\mathrm{C}(30)-\mathrm{H}(30)$ & 119.9 \\
\hline$C(36)-C(31)-C(32)$ & $117.8(2)$ \\
\hline$C(36)-C(31)-P(2)$ & $118.88(19)$ \\
\hline$C(32)-C(31)-P(2)$ & $123.3(2)$ \\
\hline$C(33)-C(32)-C(31)$ & $121.0(3)$ \\
\hline $\mathrm{C}(33)-\mathrm{C}(32)-\mathrm{H}(32)$ & 119.5 \\
\hline $\mathrm{C}(31)-\mathrm{C}(32)-\mathrm{H}(32)$ & 119.5 \\
\hline$C(34)-C(33)-C(32)$ & $120.1(3)$ \\
\hline $\mathrm{C}(34)-\mathrm{C}(33)-\mathrm{H}(33)$ & 120.0 \\
\hline $\mathrm{C}(32)-\mathrm{C}(33)-\mathrm{H}(33)$ & 120.0 \\
\hline$C(33)-C(34)-C(35)$ & $120.2(3)$ \\
\hline $\mathrm{C}(33)-\mathrm{C}(34)-\mathrm{H}(34)$ & 119.9 \\
\hline $\mathrm{C}(35)-\mathrm{C}(34)-\mathrm{H}(34)$ & 119.9 \\
\hline$C(34)-C(35)-C(36)$ & $120.2(3)$ \\
\hline $\mathrm{C}(34)-\mathrm{C}(35)-\mathrm{H}(35)$ & 119.9 \\
\hline $\mathrm{C}(36)-\mathrm{C}(35)-\mathrm{H}(35)$ & 119.9 \\
\hline$C(31)-C(36)-C(35)$ & $120.6(3)$ \\
\hline $\mathrm{C}(31)-\mathrm{C}(36)-\mathrm{H}(36)$ & 119.7 \\
\hline $\mathrm{C}(35)-\mathrm{C}(36)-\mathrm{H}(36)$ & 119.7 \\
\hline$C(38)-C(37)-H(37 A)$ & 109.5 \\
\hline $\mathrm{C}(38)-\mathrm{C}(37)-\mathrm{H}(37 \mathrm{~B})$ & 109.5 \\
\hline $\mathrm{H}(37 \mathrm{~A})-\mathrm{C}(37)-\mathrm{H}(37 \mathrm{~B})$ & 109.5 \\
\hline $\mathrm{C}(38)-\mathrm{C}(37)-\mathrm{H}(37 \mathrm{C})$ & 109.5 \\
\hline $\mathrm{H}(37 \mathrm{~A})-\mathrm{C}(37)-\mathrm{H}(37 \mathrm{C})$ & 109.5 \\
\hline $\mathrm{H}(37 \mathrm{~B})-\mathrm{C}(37)-\mathrm{H}(37 \mathrm{C})$ & 109.5 \\
\hline $\mathrm{N}(1)-\mathrm{C}(38)-\mathrm{C}(39)$ & $109.7(2)$ \\
\hline$N(1)-C(38)-C(37)$ & $122.3(2)$ \\
\hline$C(39)-C(38)-C(37)$ & $128.0(2)$ \\
\hline$C(40)-C(39)-C(38)$ & $106.0(2)$ \\
\hline $\mathrm{C}(40)-\mathrm{C}(39)-\mathrm{H}(39)$ & 127.0 \\
\hline $\mathrm{C}(38)-\mathrm{C}(39)-\mathrm{H}(39)$ & 127.0 \\
\hline $\mathrm{N}(2)-\mathrm{C}(40)-\mathrm{C}(39)$ & $108.3(2)$ \\
\hline$N(2)-C(40)-C(41)$ & $122.0(3)$ \\
\hline$C(39)-C(40)-C(41)$ & $129.7(3)$ \\
\hline $\mathrm{C}(40)-\mathrm{C}(41)-\mathrm{H}(41 \mathrm{~A})$ & 109.5 \\
\hline $\mathrm{C}(40)-\mathrm{C}(41)-\mathrm{H}(41 \mathrm{~B})$ & 109.5 \\
\hline $\mathrm{H}(41 \mathrm{~A})-\mathrm{C}(41)-\mathrm{H}(41 \mathrm{~B})$ & 109.5 \\
\hline $\mathrm{C}(40)-\mathrm{C}(41)-\mathrm{H}(41 \mathrm{C})$ & 109.5 \\
\hline $\mathrm{H}(41 \mathrm{~A})-\mathrm{C}(41)-\mathrm{H}(41 \mathrm{C})$ & 109.5 \\
\hline $\mathrm{H}(41 \mathrm{~B})-\mathrm{C}(41)-\mathrm{H}(41 \mathrm{C})$ & 109.5 \\
\hline $\mathrm{C}(43)-\mathrm{C}(42)-\mathrm{H}(42 \mathrm{~A})$ & 109.5 \\
\hline $\mathrm{C}(43)-\mathrm{C}(42)-\mathrm{H}(42 \mathrm{~B})$ & 109.5 \\
\hline $\mathrm{H}(42 \mathrm{~A})-\mathrm{C}(42)-\mathrm{H}(42 \mathrm{~B})$ & 109.5 \\
\hline $\mathrm{C}(43)-\mathrm{C}(42)-\mathrm{H}(42 \mathrm{C})$ & 109.5 \\
\hline $\mathrm{H}(42 \mathrm{~A})-\mathrm{C}(42)-\mathrm{H}(42 \mathrm{C})$ & 109.5 \\
\hline $\mathrm{H}(42 \mathrm{~B})-\mathrm{C}(42)-\mathrm{H}(42 \mathrm{C})$ & 109.5 \\
\hline$N(4)-C(43)-C(44)$ & $109.2(2)$ \\
\hline$N(4)-C(43)-C(42)$ & $121.3(2)$ \\
\hline$C(44)-C(43)-C(42)$ & $129.5(3)$ \\
\hline$C(45)-C(44)-C(43)$ & $106.4(2)$ \\
\hline
\end{tabular}




\begin{tabular}{|c|c|}
\hline $\mathrm{C}(45)-\mathrm{C}(44)-\mathrm{H}(44)$ & 126.8 \\
\hline $\mathrm{C}(43)-\mathrm{C}(44)-\mathrm{H}(44)$ & 126.8 \\
\hline$N(3)-C(45)-C(44)$ & $107.9(2)$ \\
\hline$N(3)-C(45)-C(46)$ & $122.0(3)$ \\
\hline$C(44)-C(45)-C(46)$ & $130.0(3)$ \\
\hline $\mathrm{C}(45)-\mathrm{C}(46)-\mathrm{H}(46 \mathrm{~A})$ & 109.5 \\
\hline $\mathrm{C}(45)-\mathrm{C}(46)-\mathrm{H}(46 \mathrm{~B})$ & 109.5 \\
\hline $\mathrm{H}(46 \mathrm{~A})-\mathrm{C}(46)-\mathrm{H}(46 \mathrm{~B})$ & 109.5 \\
\hline $\mathrm{C}(45)-\mathrm{C}(46)-\mathrm{H}(46 \mathrm{C})$ & 109.5 \\
\hline $\mathrm{H}(46 \mathrm{~A})-\mathrm{C}(46)-\mathrm{H}(46 \mathrm{C})$ & 109.5 \\
\hline $\mathrm{H}(46 \mathrm{~B})-\mathrm{C}(46)-\mathrm{H}(46 \mathrm{C})$ & 109.5 \\
\hline$C(49)-C(48)-C(47)$ & $111.2(5)$ \\
\hline $\mathrm{C}(49)-\mathrm{C}(48)-\mathrm{H}(48 \mathrm{~A})$ & 109.4 \\
\hline $\mathrm{C}(47)-\mathrm{C}(48)-\mathrm{H}(48 \mathrm{~A})$ & 109.4 \\
\hline $\mathrm{C}(49)-\mathrm{C}(48)-\mathrm{H}(48 \mathrm{~B})$ & 109.4 \\
\hline $\mathrm{C}(47)-\mathrm{C}(48)-\mathrm{H}(48 \mathrm{~B})$ & 109.4 \\
\hline $\mathrm{H}(48 \mathrm{~A})-\mathrm{C}(48)-\mathrm{H}(48 \mathrm{~B})$ & 108.0 \\
\hline$C(50)-C(49)-C(48)$ & $111.3(5)$ \\
\hline $\mathrm{C}(50)-\mathrm{C}(49)-\mathrm{H}(49 \mathrm{~A})$ & 109.4 \\
\hline $\mathrm{C}(48)-\mathrm{C}(49)-\mathrm{H}(49 \mathrm{~A})$ & 109.4 \\
\hline $\mathrm{C}(50)-\mathrm{C}(49)-\mathrm{H}(49 \mathrm{~B})$ & 109.4 \\
\hline $\mathrm{C}(48)-\mathrm{C}(49)-\mathrm{H}(49 \mathrm{~B})$ & 109.4 \\
\hline $\mathrm{H}(49 \mathrm{~A})-\mathrm{C}(49)-\mathrm{H}(49 \mathrm{~B})$ & 108.0 \\
\hline$C(49)-C(50)-C(51)$ & $112.4(5)$ \\
\hline $\mathrm{C}(49)-\mathrm{C}(50)-\mathrm{H}(50 \mathrm{~A})$ & 109.1 \\
\hline $\mathrm{C}(51)-\mathrm{C}(50)-\mathrm{H}(50 \mathrm{~A})$ & 109.1 \\
\hline $\mathrm{C}(49)-\mathrm{C}(50)-\mathrm{H}(50 \mathrm{~B})$ & 109.1 \\
\hline $\mathrm{C}(51)-\mathrm{C}(50)-\mathrm{H}(50 \mathrm{~B})$ & 109.1 \\
\hline $\mathrm{H}(50 \mathrm{~A})-\mathrm{C}(50)-\mathrm{H}(50 \mathrm{~B})$ & 107.9 \\
\hline$C(52)-C(51)-C(50)$ & $112.0(5)$ \\
\hline $\mathrm{C}(52)-\mathrm{C}(51)-\mathrm{H}(51 \mathrm{~A})$ & 109.2 \\
\hline $\mathrm{C}(50)-\mathrm{C}(51)-\mathrm{H}(51 \mathrm{~A})$ & 109.2 \\
\hline $\mathrm{C}(52)-\mathrm{C}(51)-\mathrm{H}(51 \mathrm{~B})$ & 109.2 \\
\hline $\mathrm{C}(50)-\mathrm{C}(51)-\mathrm{H}(51 \mathrm{~B})$ & 109.2 \\
\hline $\mathrm{H}(51 \mathrm{~A})-\mathrm{C}(51)-\mathrm{H}(51 \mathrm{~B})$ & 107.9 \\
\hline$N(3)-B(1)-N(2)$ & $105.2(2)$ \\
\hline $\mathrm{N}(3)-\mathrm{B}(1)-\mathrm{H}(1 \mathrm{~B})$ & $110.8(13)$ \\
\hline $\mathrm{N}(2)-\mathrm{B}(1)-\mathrm{H}(1 \mathrm{~B})$ & $108.5(13)$ \\
\hline $\mathrm{N}(3)-\mathrm{B}(1)-\mathrm{H}(2 \mathrm{~B})$ & $108.2(13)$ \\
\hline $\mathrm{N}(2)-\mathrm{B}(1)-\mathrm{H}(2 \mathrm{~B})$ & $113.7(13)$ \\
\hline $\mathrm{H}(1 \mathrm{~B})-\mathrm{B}(1)-\mathrm{H}(2 \mathrm{~B})$ & $110.3(19)$ \\
\hline $\mathrm{C}(38)-\mathrm{N}(1)-\mathrm{N}(2)$ & $106.8(2)$ \\
\hline $\mathrm{C}(38)-\mathrm{N}(1)-\mathrm{Rh}(1)$ & $140.67(18)$ \\
\hline $\mathrm{N}(2)-\mathrm{N}(1)-\mathrm{Rh}(1)$ & $112.11(15)$ \\
\hline $\mathrm{C}(40)-\mathrm{N}(2)-\mathrm{N}(1)$ & $109.1(2)$ \\
\hline $\mathrm{C}(40)-\mathrm{N}(2)-\mathrm{B}(1)$ & $130.3(2)$ \\
\hline $\mathrm{N}(1)-\mathrm{N}(2)-\mathrm{B}(1)$ & $119.4(2)$ \\
\hline $\mathrm{C}(45)-\mathrm{N}(3)-\mathrm{N}(4)$ & $108.8(2)$ \\
\hline $\mathrm{C}(45)-\mathrm{N}(3)-\mathrm{B}(1)$ & $132.7(2)$ \\
\hline $\mathrm{N}(4)-\mathrm{N}(3)-\mathrm{B}(1)$ & $117.6(2)$ \\
\hline $\mathrm{C}(43)-\mathrm{N}(4)-\mathrm{N}(3)$ & $107.6(2)$ \\
\hline $\mathrm{C}(43)-\mathrm{N}(4)-\mathrm{Rh}(1)$ & $135.31(18)$ \\
\hline $\mathrm{N}(3)-\mathrm{N}(4)-\mathrm{Rh}(1)$ & $116.56(15)$ \\
\hline$C(13)-P(1)-C(7)$ & $105.46(12)$ \\
\hline$C(13)-P(1)-C(1)$ & $99.93(11)$ \\
\hline$C(7)-P(1)-C(1)$ & $98.90(11)$ \\
\hline$C(13)-P(1)-R h(1)$ & $121.05(8)$ \\
\hline
\end{tabular}




\begin{tabular}{|c|c|}
\hline $\mathrm{C}(7)-\mathrm{P}(1)-\mathrm{Rh}(1)$ & $112.49(8)$ \\
\hline $\mathrm{C}(1)-\mathrm{P}(1)-\mathrm{Rh}(1)$ & $116.06(8)$ \\
\hline$C(31)-P(2)-C(25)$ & $105.53(11)$ \\
\hline$C(31)-P(2)-C(19)$ & $100.43(12)$ \\
\hline$C(25)-P(2)-C(19)$ & $99.36(12)$ \\
\hline $\mathrm{C}(31)-\mathrm{P}(2)-\mathrm{Rh}(1)$ & $113.37(8)$ \\
\hline $\mathrm{C}(25)-\mathrm{P}(2)-\mathrm{Rh}(1)$ & $110.88(8)$ \\
\hline $\mathrm{C}(19)-\mathrm{P}(2)-\mathrm{Rh}(1)$ & $124.97(8)$ \\
\hline $\mathrm{N}(4)-\mathrm{Rh}(1)-\mathrm{N}(1)$ & $79.84(8)$ \\
\hline $\mathrm{N}(4)-\mathrm{Rh}(1)-\mathrm{P}(1)$ & $92.93(6)$ \\
\hline $\mathrm{N}(1)-\mathrm{Rh}(1)-\mathrm{P}(1)$ & $164.16(6)$ \\
\hline $\mathrm{N}(4)-\mathrm{Rh}(1)-\mathrm{P}(2)$ & $170.88(6)$ \\
\hline $\mathrm{N}(1)-\mathrm{Rh}(1)-\mathrm{P}(2)$ & $92.91(6)$ \\
\hline$P(1)-R h(1)-P(2)$ & $95.43(3)$ \\
\hline$C(54)-C(53)-C(58)$ & 120.0 \\
\hline$C(54)-C(53)-C(59)$ & $119.4(4)$ \\
\hline$C(58)-C(53)-C(59)$ & $119.3(4)$ \\
\hline$C(53)-C(54)-C(55)$ & 120.0 \\
\hline $\mathrm{C}(53)-\mathrm{C}(54)-\mathrm{H}(54)$ & 120.0 \\
\hline $\mathrm{C}(55)-\mathrm{C}(54)-\mathrm{H}(54)$ & 120.0 \\
\hline$C(54)-C(55)-C(56)$ & 120.0 \\
\hline $\mathrm{C}(54)-\mathrm{C}(55)-\mathrm{H}(55)$ & 120.0 \\
\hline $\mathrm{C}(56)-\mathrm{C}(55)-\mathrm{H}(55)$ & 120.0 \\
\hline$C(55)-C(56)-C(57)$ & 120.0 \\
\hline$C(55)-C(56)-H(56)$ & 120.0 \\
\hline$C(57)-C(56)-H(56)$ & 120.0 \\
\hline$C(58)-C(57)-C(56)$ & 120.0 \\
\hline $\mathrm{C}(58)-\mathrm{C}(57)-\mathrm{H}(57)$ & 120.0 \\
\hline $\mathrm{C}(56)-\mathrm{C}(57)-\mathrm{H}(57)$ & 120.0 \\
\hline$C(57)-C(58)-C(53)$ & 120.0 \\
\hline $\mathrm{C}(57)-\mathrm{C}(58)-\mathrm{H}(58)$ & 120.0 \\
\hline $\mathrm{C}(53)-\mathrm{C}(58)-\mathrm{H}(58)$ & 120.0 \\
\hline $\mathrm{C}(53)-\mathrm{C}(59)-\mathrm{H}(59 \mathrm{~A})$ & 109.5 \\
\hline$C(53)-C(59)-H(59 B)$ & 109.5 \\
\hline $\mathrm{H}(59 \mathrm{~A})-\mathrm{C}(59)-\mathrm{H}(59 \mathrm{~B})$ & 109.5 \\
\hline$C(53)-C(59)-H(59 C)$ & 109.5 \\
\hline $\mathrm{H}(59 \mathrm{~A})-\mathrm{C}(59)-\mathrm{H}(59 \mathrm{C})$ & 109.5 \\
\hline $\mathrm{H}(59 \mathrm{~B})-\mathrm{C}(59)-\mathrm{H}(59 \mathrm{C})$ & 109.5 \\
\hline
\end{tabular}

Symmetry transformations used to generate equivalent atoms: 
Table S4. Anisotropic displacement parameters (A^2 x 10^3) for complex 2. The anisotropic displacement factor exponent takes the form:

$-2 \mathrm{pi}^{\wedge} 2\left[\mathrm{~h}^{\wedge} 2 \mathrm{a}^{\star \wedge} 2 \mathrm{U11}+\ldots+2 \mathrm{~h} \mathrm{k} \mathrm{a}^{\star} \mathrm{b}^{\star} \mathrm{U} 12\right.$ ]

\begin{tabular}{|c|c|c|c|c|c|c|}
\hline & $\mathrm{U} 11$ & $\mathrm{U} 22$ & U33 & $\mathrm{U} 23$ & $\mathrm{U} 13$ & $\mathrm{U} 12$ \\
\hline$C(1)$ & $21(1)$ & $27(1)$ & $17(1)$ & $-4(1)$ & $4(1)$ & $3(1)$ \\
\hline C (2) & $27(1)$ & $31(1)$ & $24(1)$ & $-4(1)$ & $6(1)$ & $1(1)$ \\
\hline$C(3)$ & $30(2)$ & $48(2)$ & $35(2)$ & $-13(2)$ & $2(1)$ & $-7(1)$ \\
\hline$C(4)$ & $34(2)$ & $71(2)$ & $24(2)$ & $-10(2)$ & $-4(1)$ & $1(2)$ \\
\hline$C(5)$ & $40(2)$ & $65(2)$ & $22(1)$ & $8(2)$ & $2(1)$ & $8(2)$ \\
\hline$C(6)$ & $31(2)$ & $37(2)$ & $22(1)$ & $3(1)$ & $2(1)$ & $3(1)$ \\
\hline$C(7)$ & $17(1)$ & $20(1)$ & $22(1)$ & $-2(1)$ & $2(1)$ & $-1(1)$ \\
\hline$C(8)$ & $29(2)$ & $27(1)$ & $25(1)$ & $-4(1)$ & $6(1)$ & $0(1)$ \\
\hline C (9) & $28(2)$ & $30(1)$ & $36(2)$ & $-13(1)$ & $9(1)$ & $1(1)$ \\
\hline$C(10)$ & $26(2)$ & $22(1)$ & $47(2)$ & $-6(1)$ & $1(1)$ & $2(1)$ \\
\hline C (11) & $30(2)$ & $26(1)$ & $34(2)$ & $4(1)$ & $-1(1)$ & $2(1)$ \\
\hline$C(12)$ & $23(1)$ & $26(1)$ & $26(1)$ & $-3(1)$ & $3(1)$ & $-1(1)$ \\
\hline C (13) & $25(1)$ & $24(1)$ & $18(1)$ & $2(1)$ & $2(1)$ & $-2(1)$ \\
\hline C (14) & $28(2)$ & $30(1)$ & $31(1)$ & $-2(1)$ & $11(1)$ & $-2(1)$ \\
\hline C (15) & $32(2)$ & $45(2)$ & $41(2)$ & $-1(1)$ & $18(1)$ & $-8(1)$ \\
\hline$C(16)$ & $42(2)$ & $37(2)$ & $48(2)$ & $9(2)$ & $14(2)$ & $-16(1)$ \\
\hline C (17) & $41(2)$ & $26(1)$ & $49(2)$ & $8(1)$ & $8(2)$ & $-3(1)$ \\
\hline$C(18)$ & $26(1)$ & $26(1)$ & $31(1)$ & $5(1)$ & $5(1)$ & $-1(1)$ \\
\hline C (19) & $18(1)$ & $29(1)$ & $21(1)$ & $-5(1)$ & $0(1)$ & $-3(1)$ \\
\hline$C(20)$ & $25(1)$ & $37(2)$ & $28(1)$ & $3(1)$ & $4(1)$ & $-5(1)$ \\
\hline$C(21)$ & $23(2)$ & $58(2)$ & $32(2)$ & $3(1)$ & $7(1)$ & $-11(1)$ \\
\hline C (22) & $23(2)$ & $58(2)$ & $32(2)$ & $-6(2)$ & $4(1)$ & $2(1)$ \\
\hline$C(23)$ & $28(2)$ & $44(2)$ & $46(2)$ & $-1(2)$ & $2(1)$ & $11(1)$ \\
\hline C (24) & $25(2)$ & $31(1)$ & $38(2)$ & $1(1)$ & $5(1)$ & $0(1)$ \\
\hline C (25) & $19(1)$ & $28(1)$ & $20(1)$ & $1(1)$ & $3(1)$ & $1(1)$ \\
\hline$C(26)$ & $25(1)$ & $30(1)$ & $27(1)$ & $4(1)$ & $5(1)$ & $2(1)$ \\
\hline C (27) & $31(2)$ & $40(2)$ & $34(2)$ & $12(1)$ & $7(1)$ & $3(1)$ \\
\hline C (28) & $39(2)$ & $58(2)$ & $24(1)$ & $14(2)$ & $5(1)$ & $5(2)$ \\
\hline C (29) & $37(2)$ & $56(2)$ & $21(1)$ & $-5(1)$ & $2(1)$ & $0(2)$ \\
\hline$C(30)$ & $29(2)$ & $35(2)$ & $24(1)$ & $-2(1)$ & $4(1)$ & $-1(1)$ \\
\hline$C(31)$ & $26(1)$ & $21(1)$ & $21(1)$ & $0(1)$ & $5(1)$ & $-2(1)$ \\
\hline C (32) & $33(2)$ & $28(1)$ & $33(2)$ & $-5(1)$ & $2(1)$ & $-7(1)$ \\
\hline C (33) & $49(2)$ & $29(2)$ & $45(2)$ & $-10(1)$ & $6(2)$ & $-14(1)$ \\
\hline C (34) & $62(2)$ & $18(1)$ & $48(2)$ & $-3(1)$ & $15(2)$ & $-4(1)$ \\
\hline$C(35)$ & $48(2)$ & $27(1)$ & $39(2)$ & $5(1)$ & $10(1)$ & $9(1)$ \\
\hline$C(36)$ & $30(2)$ & $25(1)$ & $26(1)$ & $1(1)$ & $5(1)$ & $-1(1)$ \\
\hline C (37) & $34(2)$ & $32(2)$ & $28(1)$ & $-6(1)$ & $10(1)$ & $2(1)$ \\
\hline C (38) & $18(1)$ & $32(1)$ & $21(1)$ & $-4(1)$ & $3(1)$ & $-1(1)$ \\
\hline C (39) & $25(1)$ & $43(2)$ & $20(1)$ & $1(1)$ & $6(1)$ & $-3(1)$ \\
\hline$C(40)$ & $21(1)$ & $37(2)$ & $24(1)$ & $8(1)$ & $5(1)$ & $-4(1)$ \\
\hline$C(41)$ & $39(2)$ & $40(2)$ & $38(2)$ & $13(1)$ & $13(1)$ & $-7(1)$ \\
\hline C (42) & $32(2)$ & $38(2)$ & $37(2)$ & $8(1)$ & $2(1)$ & $8(1)$ \\
\hline C (43) & $21(1)$ & $35(1)$ & $22(1)$ & $-2(1)$ & $5(1)$ & $3(1)$ \\
\hline$C(44)$ & $20(1)$ & $51(2)$ & $28(1)$ & $-2(1)$ & $-1(1)$ & $1(1)$ \\
\hline C (45) & $21(1)$ & $40(2)$ & $31(1)$ & $-10(1)$ & $6(1)$ & $-8(1)$ \\
\hline$C(46)$ & $33(2)$ & $55(2)$ & $58(2)$ & $-8(2)$ & $5(2)$ & $-21(2)$ \\
\hline C (47) & $61(5)$ & $31(3)$ & $82(6)$ & $-11(4)$ & $32(4)$ & $-17(3)$ \\
\hline$C(48)$ & $17(3)$ & $50(4)$ & $62(5)$ & $-12(4)$ & $4(3)$ & $-5(3)$ \\
\hline
\end{tabular}




$\begin{array}{lllrrrr}\mathrm{C}(49) & 27(3) & 43(4) & 29(3) & -7(3) & 5(2) & -6(3) \\ \mathrm{C}(50) & 18(3) & 32(3) & 42(3) & 6(3) & -9(2) & -1(2) \\ \mathrm{C}(51) & 36(4) & 52(4) & 45(4) & 0(3) & 13(3) & 11(3) \\ \mathrm{C}(52) & 60(5) & 32(4) & 110(7) & -9(4) & 33(5) & -2(3) \\ \mathrm{B}(1) & 26(2) & 21(1) & 32(2) & 1(1) & 6(1) & -2(1) \\ \mathrm{N}(1) & 19(1) & 23(1) & 20(1) & 0(1) & 4(1) & -3(1) \\ \mathrm{N}(2) & 24(1) & 24(1) & 23(1) & 2(1) & 5(1) & -3(1) \\ \mathrm{N}(3) & 22(1) & 25(1) & 25(1) & -4(1) & 5(1) & -6(1) \\ \mathrm{N}(4) & 20(1) & 24(1) & 19(1) & -1(1) & 2(1) & -3(1) \\ \mathrm{P}(1) & 19(1) & 19(1) & 17(1) & 1(1) & 3(1) & 0(1) \\ \mathrm{P}(2) & 19(1) & 19(1) & 18(1) & 0(1) & 2(1) & -1(1) \\ \mathrm{Rh}(1) & 17(1) & 17(1) & 16(1) & 0(1) & 3(1) & -1(1) \\ \end{array}$


Table S5. Hydrogen coordinates ( $x$ 10^4) and isotropic displacement parameters $\left(A^{\wedge} 2 \times 10^{\wedge} 3\right)$ for complex 2 .

\begin{tabular}{|c|c|c|c|c|}
\hline & $x$ & y & $\mathrm{z}$ & $\mathrm{U}(\mathrm{eq})$ \\
\hline $\mathrm{H}(2)$ & -1794 & 741 & 2506 & 32 \\
\hline $\mathrm{H}(3)$ & -3050 & 497 & 1689 & 45 \\
\hline $\mathrm{H}(4)$ & -3078 & 1363 & 964 & 53 \\
\hline $\mathrm{H}(5)$ & -1787 & 2446 & 1041 & 51 \\
\hline $\mathrm{H}(6)$ & -520 & 2690 & 1855 & 36 \\
\hline $\mathrm{H}(8)$ & 1044 & 894 & 2163 & 32 \\
\hline $\mathrm{H}(9)$ & 2044 & -311 & 2227 & 37 \\
\hline $\mathrm{H}(10)$ & 2361 & -991 & 3051 & 39 \\
\hline $\mathrm{H}(11)$ & 1706 & -448 & 3814 & 37 \\
\hline $\mathrm{H}(12)$ & 739 & 770 & 3758 & 30 \\
\hline $\mathrm{H}(14)$ & 2361 & 1981 & 2548 & 35 \\
\hline $\mathrm{H}(15)$ & 3479 & 3024 & 2265 & 46 \\
\hline $\mathrm{H}(16)$ & 2738 & 4309 & 2270 & 49 \\
\hline $\mathrm{H}(17)$ & 893 & 4551 & 2568 & 46 \\
\hline $\mathrm{H}(18)$ & -197 & 3519 & 2865 & 33 \\
\hline $\mathrm{H}(20)$ & 2407 & 3392 & 3674 & 36 \\
\hline $\mathrm{H}(21)$ & 4415 & 3165 & 3577 & 45 \\
\hline $\mathrm{H}(22)$ & 5415 & 2047 & 3945 & 45 \\
\hline $\mathrm{H}(23)$ & 4377 & 1133 & 4392 & 47 \\
\hline $\mathrm{H}(24)$ & 2371 & 1346 & 4499 & 38 \\
\hline $\mathrm{H}(26)$ & 151 & 1073 & 4632 & 33 \\
\hline $\mathrm{H}(27)$ & 286 & 507 & 5488 & 41 \\
\hline $\mathrm{H}(28)$ & 822 & 1272 & 6262 & 48 \\
\hline $\mathrm{H}(29)$ & 1181 & 2609 & 6176 & 46 \\
\hline $\mathrm{H}(30)$ & 1084 & 3182 & 5317 & 35 \\
\hline $\mathrm{H}(32)$ & 2168 & 3805 & 4715 & 38 \\
\hline $\mathrm{H}(33)$ & 2054 & 5168 & 4755 & 49 \\
\hline $\mathrm{H}(34)$ & 381 & 5822 & 4303 & 50 \\
\hline $\mathrm{H}(35)$ & -1174 & 5123 & 3798 & 45 \\
\hline $\mathrm{H}(36)$ & -1084 & 3752 & 3752 & 32 \\
\hline $\mathrm{H}(37 \mathrm{~A})$ & -1414 & 3223 & 4999 & 46 \\
\hline $\mathrm{H}(37 \mathrm{~B})$ & -2844 & 3309 & 4990 & 46 \\
\hline $\mathrm{H}(37 \mathrm{C})$ & -2281 & 3364 & 4435 & 46 \\
\hline $\mathrm{H}(39)$ & -3160 & 1823 & 5376 & 35 \\
\hline $\mathrm{H}(41 \mathrm{~A})$ & -3987 & 43 & 4683 & 58 \\
\hline $\mathrm{H}(41 \mathrm{~B})$ & -3573 & 259 & 5309 & 58 \\
\hline $\mathrm{H}(41 \mathrm{C})$ & -2651 & -174 & 4971 & 58 \\
\hline $\mathrm{H}(42 \mathrm{~A})$ & -2575 & 3014 & 2765 & 54 \\
\hline $\mathrm{H}(42 \mathrm{~B})$ & -4003 & 3165 & 2706 & 54 \\
\hline $\mathrm{H}(42 \mathrm{C})$ & -3432 & 2769 & 2217 & 54 \\
\hline $\mathrm{H}(44)$ & -5263 & 1598 & 2514 & 40 \\
\hline $\mathrm{H}(46 \mathrm{~A})$ & -4471 & -322 & 3157 & 73 \\
\hline $\mathrm{H}(46 \mathrm{~B})$ & -5663 & 115 & 2866 & 73 \\
\hline $\mathrm{H}(46 \mathrm{C})$ & -5271 & 193 & 3510 & 73 \\
\hline $\mathrm{H}(47 \mathrm{~A})$ & 5204 & 1648 & 6143 & 83 \\
\hline $\mathrm{H}(47 \mathrm{~B})$ & 4518 & 1375 & 5562 & 83 \\
\hline $\mathrm{H}(47 \mathrm{C})$ & 3762 & 1553 & 6048 & 83 \\
\hline $\mathrm{H}(48 \mathrm{~A})$ & 5054 & 2727 & 5539 & 52 \\
\hline $\mathrm{H}(48 \mathrm{~B})$ & 3614 & 2621 & 5417 & 52 \\
\hline
\end{tabular}




\begin{tabular}{|c|c|c|c|c|}
\hline $\mathrm{H}(49 \mathrm{~A})$ & 3473 & 2940 & 6338 & 39 \\
\hline$H(49 B)$ & 4912 & 3049 & 6458 & 39 \\
\hline $\mathrm{H}(50 \mathrm{~A})$ & 3318 & 4007 & 5725 & 39 \\
\hline $\mathrm{H}(50 \mathrm{~B})$ & 4759 & 4107 & 5823 & 39 \\
\hline $\mathrm{H}(51 \mathrm{~A})$ & 3210 & 4387 & 6626 & 53 \\
\hline $\mathrm{H}(51 \mathrm{~B})$ & 4656 & 4419 & 6761 & 53 \\
\hline $\mathrm{H}(52 \mathrm{~A})$ & 4539 & 5442 & 6055 & 98 \\
\hline $\mathrm{H}(52 \mathrm{~B})$ & 4131 & 5692 & 6622 & 98 \\
\hline $\mathrm{H}(52 \mathrm{C})$ & 3131 & 5491 & 6111 & 98 \\
\hline H ( 54$)$ & 4668 & 2954 & 5013 & 172 \\
\hline $\mathrm{H}(55)$ & 4864 & 4312 & 4898 & 212 \\
\hline $\mathrm{H}(56)$ & 4503 & 5175 & 5590 & 250 \\
\hline $\mathrm{H}(57)$ & 3946 & 4681 & 6396 & 214 \\
\hline $\mathrm{H}(58)$ & 3750 & 3323 & 6511 & 242 \\
\hline $\mathrm{H}(59 \mathrm{~A})$ & 3525 & 1910 & 5861 & 397 \\
\hline $\mathrm{H}(59 \mathrm{~B})$ & 4754 & 2081 & 6263 & 397 \\
\hline $\mathrm{H}(59 \mathrm{C})$ & 4792 & 1909 & 5633 & 397 \\
\hline $\mathrm{H}(1 \mathrm{~B})$ & $-2810(20)$ & $-18(15)$ & $3915(10)$ & $20(7)$ \\
\hline $\mathrm{H}(2 \mathrm{~B})$ & $-1420(20)$ & $409(16)$ & $3723(10)$ & $27(7)$ \\
\hline
\end{tabular}


Table s6. Torsion angles [deg] for complex 2.

\begin{tabular}{|c|c|}
\hline$C(6)-C(1)-C(2)-C(3)$ & $0.3(4)$ \\
\hline$P(1)-C(1)-C(2)-C(3)$ & $179.5(2)$ \\
\hline$C(1)-C(2)-C(3)-C(4)$ & $-0.9(4)$ \\
\hline$C(2)-C(3)-C(4)-C(5)$ & $1.3(5)$ \\
\hline$C(3)-C(4)-C(5)-C(6)$ & $-1.1(5)$ \\
\hline$C(4)-C(5)-C(6)-C(1)$ & $0.5(5)$ \\
\hline$C(2)-C(1)-C(6)-C(5)$ & $-0.1(4)$ \\
\hline$P(1)-C(1)-C(6)-C(5)$ & $-179.3(2)$ \\
\hline$C(12)-C(7)-C(8)-C(9)$ & $-1.3(4)$ \\
\hline$P(1)-C(7)-C(8)-C(9)$ & $-176.2(2)$ \\
\hline$C(7)-C(8)-C(9)-C(10)$ & $1.2(4)$ \\
\hline$C(8)-C(9)-C(10)-C(11)$ & $-0.5(4)$ \\
\hline$C(9)-C(10)-C(11)-C(12)$ & $-0.2(4)$ \\
\hline$C(8)-C(7)-C(12)-C(11)$ & $0.6(4)$ \\
\hline $\mathrm{P}(1)-\mathrm{C}(7)-\mathrm{C}(12)-\mathrm{C}(11)$ & $175.7(2)$ \\
\hline$C(10)-C(11)-C(12)-C(7)$ & $0.1(4)$ \\
\hline$C(18)-C(13)-C(14)-C(15)$ & $-0.3(4)$ \\
\hline$P(1)-C(13)-C(14)-C(15)$ & $175.5(2)$ \\
\hline$C(13)-C(14)-C(15)-C(16)$ & $-0.4(5)$ \\
\hline$C(14)-C(15)-C(16)-C(17)$ & $0.5(5)$ \\
\hline$C(15)-C(16)-C(17)-C(18)$ & $0.2(5)$ \\
\hline$C(16)-C(17)-C(18)-C(13)$ & $-0.9(5)$ \\
\hline$C(14)-C(13)-C(18)-C(17)$ & $1.0(4)$ \\
\hline $\mathrm{P}(1)-\mathrm{C}(13)-\mathrm{C}(18)-\mathrm{C}(17)$ & $-175.2(2)$ \\
\hline$C(24)-C(19)-C(20)-C(21)$ & $-0.4(4)$ \\
\hline$P(2)-C(19)-C(20)-C(21)$ & $-179.8(2)$ \\
\hline$C(19)-C(20)-C(21)-C(22)$ & $-0.2(4)$ \\
\hline$C(20)-C(21)-C(22)-C(23)$ & $0.9(5)$ \\
\hline$C(21)-C(22)-C(23)-C(24)$ & $-1.0(5)$ \\
\hline$C(22)-C(23)-C(24)-C(19)$ & $0.4(5)$ \\
\hline$C(20)-C(19)-C(24)-C(23)$ & $0.3(4)$ \\
\hline$P(2)-C(19)-C(24)-C(23)$ & $179.7(2)$ \\
\hline$C(30)-C(25)-C(26)-C(27)$ & $-1.3(4)$ \\
\hline$P(2)-C(25)-C(26)-C(27)$ & $176.6(2)$ \\
\hline$C(25)-C(26)-C(27)-C(28)$ & $0.6(4)$ \\
\hline$C(26)-C(27)-C(28)-C(29)$ & $0.7(5)$ \\
\hline$C(27)-C(28)-C(29)-C(30)$ & $-1 \cdot 3(5)$ \\
\hline$C(26)-C(25)-C(30)-C(29)$ & $0.7(4)$ \\
\hline$P(2)-C(25)-C(30)-C(29)$ & $-177.1(2)$ \\
\hline$C(28)-C(29)-C(30)-C(25)$ & $0.6(5)$ \\
\hline$C(36)-C(31)-C(32)-C(33)$ & $1.1(4)$ \\
\hline$P(2)-C(31)-C(32)-C(33)$ & $179.6(2)$ \\
\hline$C(31)-C(32)-C(33)-C(34)$ & $-0.3(5)$ \\
\hline$C(32)-C(33)-C(34)-C(35)$ & $-0.6(5)$ \\
\hline$C(33)-C(34)-C(35)-C(36)$ & $0.6(5)$ \\
\hline$C(32)-C(31)-C(36)-C(35)$ & $-1.1(4)$ \\
\hline$P(2)-C(31)-C(36)-C(35)$ & $-179.6(2)$ \\
\hline$C(34)-C(35)-C(36)-C(31)$ & $0.2(4)$ \\
\hline $\mathrm{N}(1)-\mathrm{C}(38)-\mathrm{C}(39)-\mathrm{C}(40)$ & $-1.2(3)$ \\
\hline$C(37)-C(38)-C(39)-C(40)$ & $176.2(3)$ \\
\hline$C(38)-C(39)-C(40)-N(2)$ & $0.2(3)$ \\
\hline$C(38)-C(39)-C(40)-C(41)$ & $-179.5(3)$ \\
\hline$N(4)-C(43)-C(44)-C(45)$ & $0.5(3)$ \\
\hline$C(42)-C(43)-C(44)-C(45)$ & $-178.9(3)$ \\
\hline
\end{tabular}




\begin{tabular}{|c|c|}
\hline$C(43)-C(44)-C(45)-N(3)$ & $-0.2(3)$ \\
\hline$C(43)-C(44)-C(45)-C(46)$ & $176.6(3)$ \\
\hline$C(47)-C(48)-C(49)-C(50)$ & $-179.7(5)$ \\
\hline$C(48)-C(49)-C(50)-C(51)$ & $-177.9(5)$ \\
\hline$C(49)-C(50)-C(51)-C(52)$ & $174.7(6)$ \\
\hline $\mathrm{C}(39)-\mathrm{C}(38)-\mathrm{N}(1)-\mathrm{N}(2)$ & $1.8(3)$ \\
\hline $\mathrm{C}(37)-\mathrm{C}(38)-\mathrm{N}(1)-\mathrm{N}(2)$ & $-175.8(2)$ \\
\hline $\mathrm{C}(39)-\mathrm{C}(38)-\mathrm{N}(1)-\mathrm{Rh}(1)$ & $-169.9(2)$ \\
\hline $\mathrm{C}(37)-\mathrm{C}(38)-\mathrm{N}(1)-\mathrm{Rh}(1)$ & $12.5(4)$ \\
\hline $\mathrm{C}(39)-\mathrm{C}(40)-\mathrm{N}(2)-\mathrm{N}(1)$ & $0.9(3)$ \\
\hline $\mathrm{C}(41)-\mathrm{C}(40)-\mathrm{N}(2)-\mathrm{N}(1)$ & $-179.4(2)$ \\
\hline $\mathrm{C}(39)-\mathrm{C}(40)-\mathrm{N}(2)-\mathrm{B}(1)$ & $-165.8(3)$ \\
\hline $\mathrm{C}(41)-\mathrm{C}(40)-\mathrm{N}(2)-\mathrm{B}(1)$ & $13.9(4)$ \\
\hline $\mathrm{C}(38)-\mathrm{N}(1)-\mathrm{N}(2)-\mathrm{C}(40)$ & $-1.7(3)$ \\
\hline $\mathrm{Rh}(1)-\mathrm{N}(1)-\mathrm{N}(2)-\mathrm{C}(40)$ & $172.64(16)$ \\
\hline $\mathrm{C}(38)-\mathrm{N}(1)-\mathrm{N}(2)-\mathrm{B}(1)$ & $166.7(2)$ \\
\hline $\mathrm{Rh}(1)-\mathrm{N}(1)-\mathrm{N}(2)-\mathrm{B}(1)$ & $-19.0(3)$ \\
\hline $\mathrm{N}(3)-\mathrm{B}(1)-\mathrm{N}(2)-\mathrm{C}(40)$ & $114.4(3)$ \\
\hline $\mathrm{N}(3)-\mathrm{B}(1)-\mathrm{N}(2)-\mathrm{N}(1)$ & $-51.2(3)$ \\
\hline $\mathrm{C}(44)-\mathrm{C}(45)-\mathrm{N}(3)-\mathrm{N}(4)$ & $-0.2(3)$ \\
\hline $\mathrm{C}(46)-\mathrm{C}(45)-\mathrm{N}(3)-\mathrm{N}(4)$ & $-177.3(3)$ \\
\hline $\mathrm{C}(44)-\mathrm{C}(45)-\mathrm{N}(3)-\mathrm{B}(1)$ & $168.4(3)$ \\
\hline $\mathrm{C}(46)-\mathrm{C}(45)-\mathrm{N}(3)-\mathrm{B}(1)$ & $-8.8(5)$ \\
\hline $\mathrm{N}(2)-\mathrm{B}(1)-\mathrm{N}(3)-\mathrm{C}(45)$ & $-106.5(3)$ \\
\hline $\mathrm{N}(2)-\mathrm{B}(1)-\mathrm{N}(3)-\mathrm{N}(4)$ & $61.3(3)$ \\
\hline $\mathrm{C}(44)-\mathrm{C}(43)-\mathrm{N}(4)-\mathrm{N}(3)$ & $-0.6(3)$ \\
\hline $\mathrm{C}(42)-\mathrm{C}(43)-\mathrm{N}(4)-\mathrm{N}(3)$ & $178.8(2)$ \\
\hline $\mathrm{C}(44)-\mathrm{C}(43)-\mathrm{N}(4)-\mathrm{Rh}(1)$ & $-171.93(19)$ \\
\hline $\mathrm{C}(42)-\mathrm{C}(43)-\mathrm{N}(4)-\mathrm{Rh}(1)$ & $7.5(4)$ \\
\hline $\mathrm{C}(45)-\mathrm{N}(3)-\mathrm{N}(4)-\mathrm{C}(43)$ & $0.5(3)$ \\
\hline $\mathrm{B}(1)-\mathrm{N}(3)-\mathrm{N}(4)-\mathrm{C}(43)$ & $-170.0(2)$ \\
\hline $\mathrm{C}(45)-\mathrm{N}(3)-\mathrm{N}(4)-\mathrm{Rh}(1)$ & $173.70(16)$ \\
\hline $\mathrm{B}(1)-\mathrm{N}(3)-\mathrm{N}(4)-\mathrm{Rh}(1)$ & $3.2(3)$ \\
\hline $\mathrm{C}(14)-\mathrm{C}(13)-\mathrm{P}(1)-\mathrm{C}(7)$ & $10.8(3)$ \\
\hline $\mathrm{C}(18)-\mathrm{C}(13)-\mathrm{P}(1)-\mathrm{C}(7)$ & $-173.28(19)$ \\
\hline $\mathrm{C}(14)-\mathrm{C}(13)-\mathrm{P}(1)-\mathrm{C}(1)$ & $-91.4(2)$ \\
\hline$C(18)-C(13)-P(1)-C(1)$ & $84.5(2)$ \\
\hline $\mathrm{C}(14)-\mathrm{C}(13)-\mathrm{P}(1)-\mathrm{Rh}(1)$ & $139.9(2)$ \\
\hline $\mathrm{C}(18)-\mathrm{C}(13)-\mathrm{P}(1)-\mathrm{Rh}(1)$ & $-44.3(2)$ \\
\hline $\mathrm{C}(12)-\mathrm{C}(7)-\mathrm{P}(1)-\mathrm{C}(13)$ & $120.3(2)$ \\
\hline$C(8)-C(7)-P(1)-C(13)$ & $-64.8(2)$ \\
\hline $\mathrm{C}(12)-\mathrm{C}(7)-\mathrm{P}(1)-\mathrm{C}(1)$ & $-136.7(2)$ \\
\hline$C(8)-C(7)-P(1)-C(1)$ & $38.1(2)$ \\
\hline $\mathrm{C}(12)-\mathrm{C}(7)-\mathrm{P}(1)-\mathrm{Rh}(1)$ & $-13.6(2)$ \\
\hline $\mathrm{C}(8)-\mathrm{C}(7)-\mathrm{P}(1)-\mathrm{Rh}(1)$ & $161.24(19)$ \\
\hline$C(6)-C(1)-P(1)-C(13)$ & $-13 \cdot 2(3)$ \\
\hline $\mathrm{C}(2)-\mathrm{C}(1)-\mathrm{P}(1)-\mathrm{C}(13)$ & $167.5(2)$ \\
\hline $\mathrm{C}(6)-\mathrm{C}(1)-\mathrm{P}(1)-\mathrm{C}(7)$ & $-120.8(2)$ \\
\hline$C(2)-C(1)-P(1)-C(7)$ & $60.0(2)$ \\
\hline $\mathrm{C}(6)-\mathrm{C}(1)-\mathrm{P}(1)-\mathrm{Rh}(1)$ & $118.7(2)$ \\
\hline $\mathrm{C}(2)-\mathrm{C}(1)-\mathrm{P}(1)-\mathrm{Rh}(1)$ & $-60.5(2)$ \\
\hline$C(36)-C(31)-P(2)-C(25)$ & $-119.4(2)$ \\
\hline $\mathrm{C}(32)-\mathrm{C}(31)-\mathrm{P}(2)-\mathrm{C}(25)$ & $62.2(3)$ \\
\hline$C(36)-C(31)-P(2)-C(19)$ & $137.7(2)$ \\
\hline$C(32)-C(31)-P(2)-C(19)$ & $-40.7(2)$ \\
\hline $\mathrm{C}(36)-\mathrm{C}(31)-\mathrm{P}(2)-\mathrm{Rh}(1)$ & $2.1(2)$ \\
\hline $\mathrm{C}(32)-\mathrm{C}(31)-\mathrm{P}(2)-\mathrm{Rh}(1)$ & $-176.3(2)$ \\
\hline
\end{tabular}




\begin{tabular}{|c|c|}
\hline$C(30)-C(25)-P(2)-C(31)$ & $-16.4(3)$ \\
\hline$C(26)-C(25)-P(2)-C(31)$ & $165.8(2)$ \\
\hline$C(30)-C(25)-P(2)-C(19)$ & $87.3(2)$ \\
\hline$C(26)-C(25)-P(2)-C(19)$ & $-90.5(2)$ \\
\hline$C(30)-C(25)-P(2)-R h(1)$ & $-139.5(2)$ \\
\hline$C(26)-C(25)-P(2)-R h(1)$ & $42.7(2)$ \\
\hline$C(24)-C(19)-P(2)-C(31)$ & $147.2(2)$ \\
\hline$C(20)-C(19)-P(2)-C(31)$ & $-33.4(2)$ \\
\hline$C(24)-C(19)-P(2)-C(25)$ & $39.4(2)$ \\
\hline$C(20)-C(19)-P(2)-C(25)$ & $-141.2(2)$ \\
\hline$C(24)-C(19)-P(2)-R h(1)$ & $-84.4(2)$ \\
\hline$C(20)-C(19)-P(2)-R h(1)$ & $95.0(2)$ \\
\hline $\mathrm{C}(43)-\mathrm{N}(4)-\mathrm{Rh}(1)-\mathrm{N}(1)$ & $114.9(3)$ \\
\hline $\mathrm{N}(3)-\mathrm{N}(4)-\mathrm{Rh}(1)-\mathrm{N}(1)$ & $-55.89(17)$ \\
\hline $\mathrm{C}(43)-\mathrm{N}(4)-\mathrm{Rh}(1)-\mathrm{P}(1)$ & $-79.3(2)$ \\
\hline $\mathrm{N}(3)-\mathrm{N}(4)-\mathrm{Rh}(1)-\mathrm{P}(1)$ & $109.91(16)$ \\
\hline $\mathrm{C}(43)-\mathrm{N}(4)-\mathrm{Rh}(1)-\mathrm{P}(2)$ & $77.1(5)$ \\
\hline $\mathrm{N}(3)-\mathrm{N}(4)-\mathrm{Rh}(1)-\mathrm{P}(2)$ & $-93.6(4)$ \\
\hline $\mathrm{C}(38)-\mathrm{N}(1)-\mathrm{Rh}(1)-\mathrm{N}(4)$ & $-125.5(3)$ \\
\hline $\mathrm{N}(2)-\mathrm{N}(1)-\mathrm{Rh}(1)-\mathrm{N}(4)$ & $63.08(16)$ \\
\hline $\mathrm{C}(38)-\mathrm{N}(1)-\mathrm{Rh}(1)-\mathrm{P}(1)$ & $170.69(19)$ \\
\hline $\mathrm{N}(2)-\mathrm{N}(1)-\mathrm{Rh}(1)-\mathrm{P}(1)$ & $-0.7(3)$ \\
\hline $\mathrm{C}(38)-\mathrm{N}(1)-\mathrm{Rh}(1)-\mathrm{P}(2)$ & $48.9(3)$ \\
\hline $\mathrm{N}(2)-\mathrm{N}(1)-\mathrm{Rh}(1)-\mathrm{P}(2)$ & $-122.49(15)$ \\
\hline $\mathrm{C}(13)-\mathrm{P}(1)-\mathrm{Rh}(1)-\mathrm{N}(4)$ & $133.82(11)$ \\
\hline $\mathrm{C}(7)-\mathrm{P}(1)-\mathrm{Rh}(1)-\mathrm{N}(4)$ & $-100.30(10)$ \\
\hline $\mathrm{C}(1)-\mathrm{P}(1)-\mathrm{Rh}(1)-\mathrm{N}(4)$ & $12.58(11)$ \\
\hline $\mathrm{C}(13)-\mathrm{P}(1)-\mathrm{Rh}(1)-\mathrm{N}(1)$ & $-164.0(2)$ \\
\hline $\mathrm{C}(7)-\mathrm{P}(1)-\mathrm{Rh}(1)-\mathrm{N}(1)$ & $-38.1(2)$ \\
\hline $\mathrm{C}(1)-\mathrm{P}(1)-\mathrm{Rh}(1)-\mathrm{N}(1)$ & $74.8(2)$ \\
\hline$C(13)-P(1)-R h(1)-P(2)$ & $-42.53(10)$ \\
\hline $\mathrm{C}(7)-\mathrm{P}(1)-\mathrm{Rh}(1)-\mathrm{P}(2)$ & $83.35(9)$ \\
\hline $\mathrm{C}(1)-\mathrm{P}(1)-\mathrm{Rh}(1)-\mathrm{P}(2)$ & $-163.77(9)$ \\
\hline $\mathrm{C}(31)-\mathrm{P}(2)-\mathrm{Rh}(1)-\mathrm{N}(4)$ & $-56.4(4)$ \\
\hline$C(25)-P(2)-R h(1)-N(4)$ & $62.1(4)$ \\
\hline $\mathrm{C}(19)-\mathrm{P}(2)-\mathrm{Rh}(1)-\mathrm{N}(4)$ & $-179(19)$ \\
\hline $\mathrm{C}(31)-\mathrm{P}(2)-\mathrm{Rh}(1)-\mathrm{N}(1)$ & $-93.46(10)$ \\
\hline $\mathrm{C}(25)-\mathrm{P}(2)-\mathrm{Rh}(1)-\mathrm{N}(1)$ & $25.03(10)$ \\
\hline $\mathrm{C}(19)-\mathrm{P}(2)-\mathrm{Rh}(1)-\mathrm{N}(1)$ & $143.67(12)$ \\
\hline $\mathrm{C}(31)-\mathrm{P}(2)-\mathrm{Rh}(1)-\mathrm{P}(1)$ & $100.02(9)$ \\
\hline$C(25)-P(2)-R h(1)-P(1)$ & $-141.49(9)$ \\
\hline$C(19)-P(2)-R h(1)-P(1)$ & $-22.85(11)$ \\
\hline$C(58)-C(53)-C(54)-C(55)$ & 0.0 \\
\hline$C(59)-C(53)-C(54)-C(55)$ & $-166.8(17)$ \\
\hline$C(53)-C(54)-C(55)-C(56)$ & 0.0 \\
\hline$C(54)-C(55)-C(56)-C(57)$ & 0.0 \\
\hline$C(55)-C(56)-C(57)-C(58)$ & 0.0 \\
\hline$C(56)-C(57)-C(58)-C(53)$ & 0.0 \\
\hline$C(54)-C(53)-C(58)-C(57)$ & 0.0 \\
\hline$C(59)-C(53)-C(58)-C(57)$ & $166.8(17)$ \\
\hline
\end{tabular}


Figure S3. Crystal Structure of Complex 3 - Configuration represented in paper.

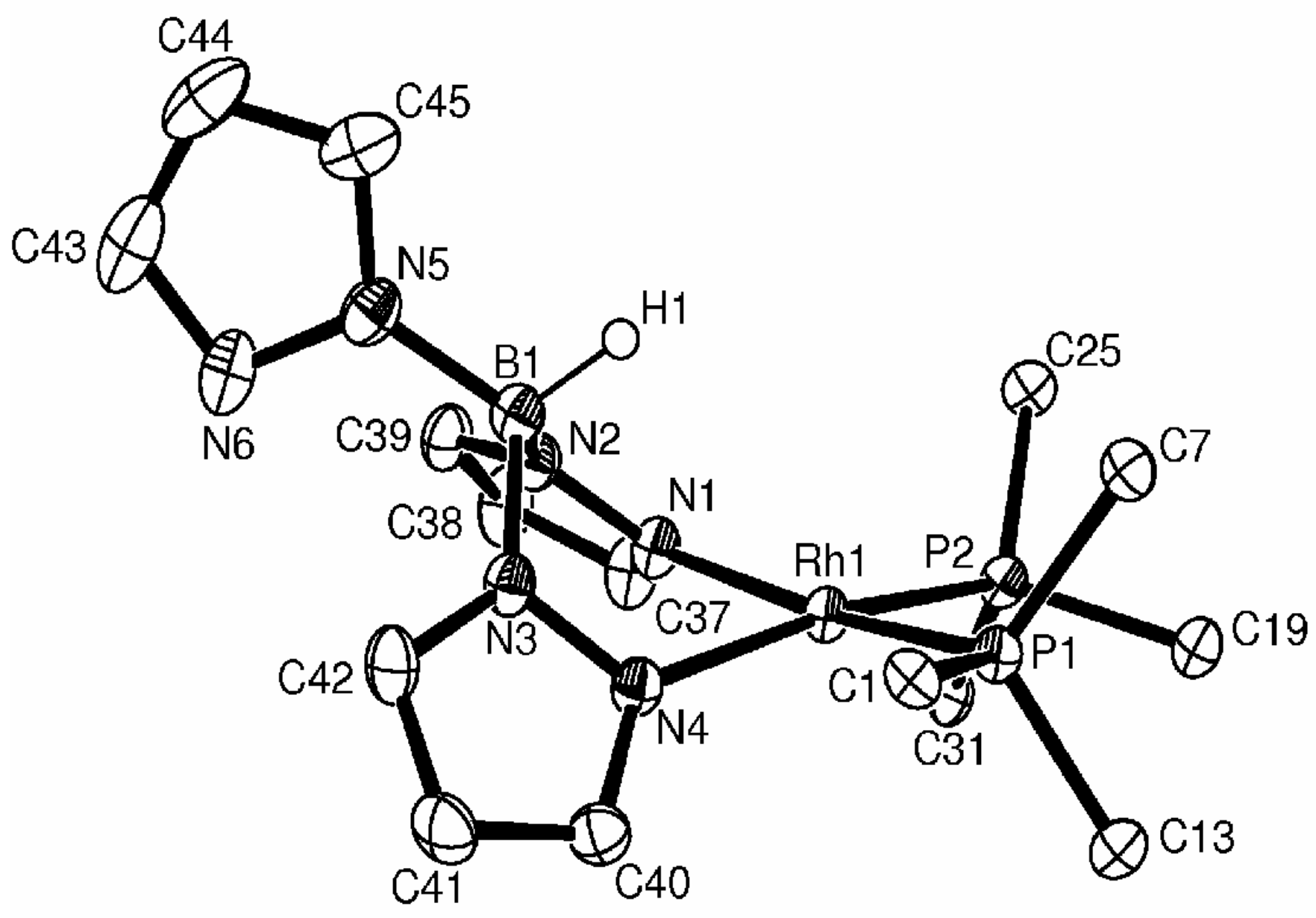


Figure S4. Crystal Structure of Complex 2 - Including all atoms.

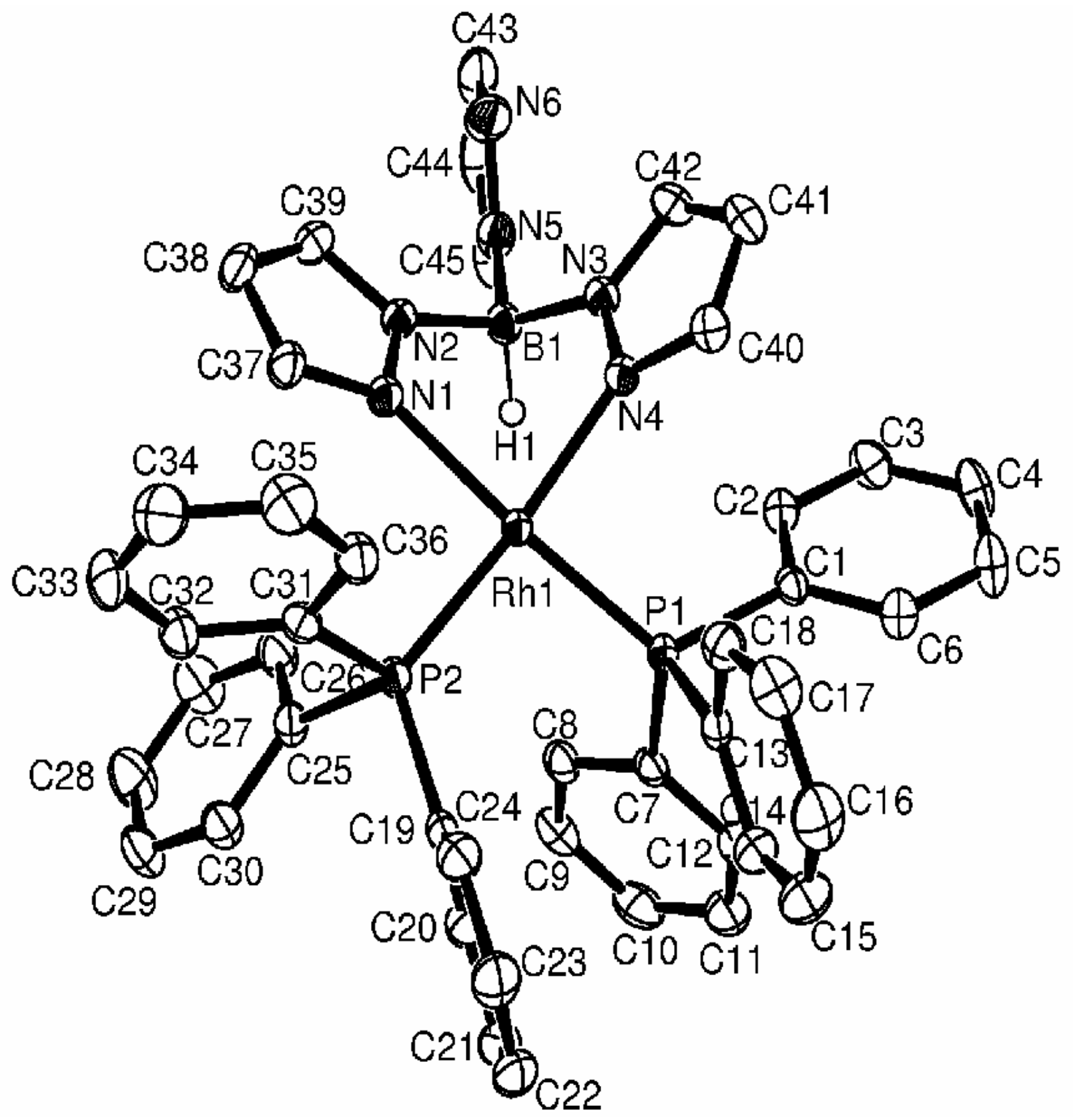




\section{Experimental}

\section{Data Collection}

A red irregular crystal of $\mathrm{C}_{45} \mathrm{H}_{40} \mathrm{BN}_{6} \mathrm{P}_{2}$ Rh. $\mathrm{C}_{7} \mathrm{H}_{8}$ having approximate dimensions of $0.20 \times 0.20 \times 0.10$ $\mathrm{mm}$ was mounted on a glass fiber. All measurements were made on a Bruker X8 APEX diffractometer with graphite monochromated Mo-Ka radiation.

The data were collected at a temperature of $-100.0 \pm 0.1^{\circ} \mathrm{C}$ to a maximum $2 \theta$ value of $55.8^{\circ}$. Data were collected in a series of $\phi$ and $\omega$ scans in $0.50^{\circ}$ oscillations with 5.0 second exposures. The crystal-todetector distance was $38.01 \mathrm{~mm}$.

\section{Data Reduction}

Of the 77370 reflections that were collected, 10812 were unique $\left(R_{i n t}=0.042\right)$; equivalent reflections were merged. Data were collected and integrated using the Bruker SAINT ${ }^{1}$ software package. The linear absorption coefficient, $\mu$, for Mo-K $\alpha$ radiation is $4.86 \mathrm{~cm}^{-1}$. Data were corrected for absorption effects using the multi-scan technique (SADABS ${ }^{2}$ ), with minimum and maximum transmission coefficients of 0.858 and 0.953, respectively. The data were corrected for Lorentz and polarization effects.

\section{$\underline{\text { Structure Solution and Refinement }}$}

The structure was solved by direct methods ${ }^{3}$. All non-hydrogen atoms were refined anisotropically. All hydrogen atoms except $\mathrm{H} 1$ (found in a difference map and refined isotropically) were included in calculated positions but not refined. The final cycle of full-matrix least-squares refinement ${ }^{4}$ on $\mathrm{F}^{2}$ was based on 10812 reflections and 603 variable parameters and converged (largest parameter shift was 0.00 times its esd) with unweighted and weighted agreement factors of:

$$
\begin{gathered}
\mathrm{R} 1=\Sigma\|\mathrm{Fo}|-| \mathrm{Fc}\| / \Sigma|\mathrm{Fo}|=0.036 \\
\mathrm{wR} 2=\left[\Sigma\left(\mathrm{w}\left(\mathrm{Fo}^{2}-\mathrm{Fc}^{2}\right)^{2}\right) / \Sigma \mathrm{w}\left(\mathrm{Fo}^{2}\right)^{2}\right]^{1 / 2}=0.064
\end{gathered}
$$

The standard deviation of an observation of unit weight ${ }^{5}$ was 1.11 . The weighting scheme was 
based on counting statistics. The maximum and minimum peaks on the final difference Fourier map corresponded to 0.37 and $-0.35 \mathrm{e}^{-} / \AA^{3}$, respectively.

Neutral atom scattering factors were taken from Cromer and Waber 6 . Anomalous dispersion effects were included in Fcalc ${ }^{7}$; the values for $\Delta f^{\prime}$ and $\Delta f^{\prime \prime}$ were those of Creagh and McAuley ${ }^{8}$. The values for the mass attenuation coefficients are those of Creagh and Hubbell ${ }^{9}$. All refinements were performed using the SHELXTL 10 crystallographic software package of Bruker-AXS.

\section{References}

(1) SAINT. Version 7.03A. Bruker AXS Inc., Madison, Wisconsin, USA. (1997-2003).

(2) SADABS. Bruker Nonius area detector scaling and absorption correction - V2.10, Bruker AXS Inc., Madison, Wisconsin, USA (2003).

(3) SIR97 - Altomare A., Burla M.C., Camalli M., Cascarano G.L., Giacovazzo C. , Guagliardi A., Moliterni A.G.G., Polidori G.,Spagna R. (1999) J. Appl. Cryst. 32, 115-119.

(4) Least Squares function minimized:

$$
\Sigma w\left(\mathrm{~F}_{\mathrm{o}}{ }^{2}-\mathrm{F}_{\mathrm{C}}\right)^{2}
$$

(5) Standard deviation of an observation of unit weight:

$$
\begin{aligned}
& {\left[\Sigma w\left(\mathrm{~F}_{\mathrm{O}}{ }^{2}-\mathrm{F}_{\mathrm{C}}{ }^{2}\right)^{2} /\left(\mathrm{N}_{\mathrm{O}}-\mathrm{N}_{\mathrm{V}}\right)\right]^{1 / 2}} \\
& \text { where: } \mathrm{N}_{\mathrm{O}}=\text { number of observations } \\
& \qquad \mathrm{N}_{\mathrm{V}}=\text { number of variables }
\end{aligned}
$$

(6) Cromer, D. T. \& Waber, J. T.; "International Tables for X-ray Crystallography", Vol. IV, The Kynoch Press, Birmingham, England, Table 2.2 A (1974).

(7) Ibers, J. A. \& Hamilton, W. C.; Acta Crystallogr., 17, 781 (1964).

(8) Creagh, D. C. \& McAuley, W.J .; "International Tables for Crystallography", Vol C, (A.J.C. Wilson, ed.), Kluwer Academic Publishers, Boston, Table 4.2.6.8, pages 219-222 (1992).

(9) Creagh, D. C. \& Hubbell, J.H..; "International Tables for Crystallography", Vol C, (A.J.C. Wilson, ed.), Kluwer Academic Publishers, Boston, Table 4.2.4.3, pages 200-206 (1992).

(10) SHELXTL Version 5.1. Bruker AXS Inc., Madision, Wisconsin, USA. (1997). 
Table S7. Crystal Data and Structure Analysis Details for Complex 3.

\author{
A. Crystal Data
}

Empirical Formula
Formula Weight
Crystal Color, Habit
Crystal Dimensions
Crystal System
Lattice Type
Lattice Parameters

$\mathrm{C}_{52} \mathrm{H}_{48} \mathrm{BN}_{6} \mathrm{P}_{2} \mathrm{RhFeP}$

932.62

red, irregular

$0.20 \times 0.20 \times 0.10 \mathrm{~mm}$

triclinic

primitive

$a=12.2490(9) \AA$

$\mathrm{b}=13.637(1) \AA$

$\mathrm{c}=14.971(1) \AA$

$\alpha=112.004(3)^{\circ}$

$\beta=98.051(3)^{\circ}$

$\gamma=90.387(3)^{\circ}$

$\mathrm{V}=2291.0(3) \AA^{3}$

Space Group

$P-1(\# 2)$

$Z$ value

2

$\mathrm{D}_{\text {calc }}$

$1.352 \mathrm{~g} / \mathrm{cm}^{3}$

$F_{000}$

964.00

$\mu(\mathrm{MoK} \alpha)$

$4.86 \mathrm{~cm}^{-1}$ 
B. Intensity Measurements

Diffractometer

Radiation

Data Images

Detector Position

$2 \theta \max$

No. of Reflections Measured

Corrections
Bruker X8 APEX

$\operatorname{MoK} \alpha(\lambda=0.71073 \AA)$

graphite monochromated

3504 exposures @ 5.0 seconds

$38.01 \mathrm{~mm}$

$55.8^{\circ}$

Total: 77370

Unique: $10812\left(R_{\text {int }}=0.042\right)$

Absorption $\left(T_{\text {min }}=0.858, T_{\max }=0.953\right)$ Lorentz-polarization 
C. Structure Solution and Refinement

\begin{tabular}{|c|c|}
\hline Structure Solution & Direct Methods (SIR97) \\
\hline Refinement & Full-matrix least-squares on $\mathrm{F}^{2}$ \\
\hline Function Minimized & $\Sigma w\left(F o^{2}-F c^{2}\right)^{2}$ \\
\hline Least Squares Weights & $w=1 /\left(\sigma^{2}\left(\mathrm{Fo}^{2}\right)+(0.0287 \mathrm{P})^{2}+0.3727 \mathrm{P}\right)$ \\
\hline Anomalous Dispersion & All non-hydrogen atoms \\
\hline No. Observations $(I>0.00 \sigma(I))$ & 10812 \\
\hline No. Variables & 603 \\
\hline Reflection/Parameter Ratio & 17.93 \\
\hline Residuals (refined on $\mathrm{F}^{2}$, all data): $\mathrm{R} 1$; wR2 & $0.036 ; 0.064$ \\
\hline Goodness of Fit Indicator & 1.11 \\
\hline No. Observations $(I>2.00 \sigma(I))$ & 9032 \\
\hline Residuals (refined on F): R1; wR2 & $0.027 ; 0.063$ \\
\hline Max Shift/Error in Final Cycle & 0.00 \\
\hline Maximum peak in Final Diff. Map & $0.37 \mathrm{e}^{-/} / \AA^{3}$ \\
\hline Minimum peak in Final Diff. Map & $-0.35 e^{-} / \AA^{3}$ \\
\hline
\end{tabular}


Table S8. Atomic coordinates $\left(x 1^{\wedge} 4\right)$ and equivalent isotropic displacement parameters $\left(A^{\wedge} 2 \times 10^{\wedge} 3\right)$ for complex 3 .

$\mathrm{U}(e q)$ is defined as one third of the trace of the orthogonalized Uij tensor.

\begin{tabular}{|c|c|c|c|c|}
\hline & $x$ & y & z & $\mathrm{U}(\mathrm{eq})$ \\
\hline$C(1)$ & $1746(1)$ & $1039(1)$ & $3272(1)$ & $19(1)$ \\
\hline$C(2)$ & $1156(1)$ & $1127(1)$ & $4032(1)$ & $23(1)$ \\
\hline$C(3)$ & $1325(2)$ & $452(1)$ & $4530(1)$ & $29(1)$ \\
\hline$C(4)$ & $2096(2)$ & $-314(1)$ & $4288(1)$ & $34(1)$ \\
\hline$C(5)$ & $2708(2)$ & $-386(1)$ & $3558(1)$ & $34(1)$ \\
\hline$C(6)$ & $2535(1)$ & $286(1)$ & $3049(1)$ & $25(1)$ \\
\hline$C(7)$ & $2385(1)$ & $3099(1)$ & $3637(1)$ & $20(1)$ \\
\hline$C(8)$ & $1879(1)$ & $4022(1)$ & $4136(1)$ & $23(1)$ \\
\hline$C(9)$ & $2465(2)$ & $4825(1)$ & $4949(1)$ & $30(1)$ \\
\hline$C(10)$ & $3557(2)$ & $4716(2)$ & $5270(1)$ & $36(1)$ \\
\hline$C(11)$ & $4066(2)$ & $3801(2)$ & $4790(1)$ & $35(1)$ \\
\hline$C(12)$ & $3478(1)$ & 2991 (1) & $3990(1)$ & $26(1)$ \\
\hline$C(13)$ & 2279 (1) & $1426(1)$ & $1632(1)$ & $20(1)$ \\
\hline$C(14)$ & $3375(1)$ & $1669(1)$ & $1598(1)$ & $27(1)$ \\
\hline$C(15)$ & $3861(2)$ & $1111(2)$ & $781(2)$ & $38(1)$ \\
\hline$C(16)$ & $3268(2)$ & $299(2)$ & $-9(1)$ & $38(1)$ \\
\hline$C(17)$ & $2173(2)$ & $54(2)$ & $6(1)$ & $32(1)$ \\
\hline$C(18)$ & $1680(1)$ & $624(1)$ & $816(1)$ & $25(1)$ \\
\hline$C(19)$ & $1584(1)$ & $3717(1)$ & $1421(1)$ & $21(1)$ \\
\hline$C(20)$ & $2442(1)$ & $4369(1)$ & $2112(1)$ & $26(1)$ \\
\hline$C(21)$ & $3472(1)$ & $4480(2)$ & $1851(1)$ & $32(1)$ \\
\hline$C(22)$ & $3653(2)$ & $3938(2)$ & $902(1)$ & $34(1)$ \\
\hline$C(23)$ & $2823(2)$ & $3278(2)$ & $215(1)$ & $33(1)$ \\
\hline$C(24)$ & $1792(1)$ & $3167(1)$ & $471(1)$ & $28(1)$ \\
\hline C (25) & $-2(1)$ & $4976(1)$ & $2488(1)$ & $22(1)$ \\
\hline$C(26)$ & $-610(1)$ & $5214(1)$ & $3256(1)$ & $29(1)$ \\
\hline$C(27)$ & $-835(2)$ & $6262(2)$ & $3767(2)$ & $40(1)$ \\
\hline$C(28)$ & $-464(2)$ & $7067(2)$ & $3512(2)$ & $42(1)$ \\
\hline$C(29)$ & $148(2)$ & $6844(1)$ & $2760(1)$ & $34(1)$ \\
\hline$C(30)$ & $381(1)$ & $5804(1)$ & 2251 (1) & $27(1)$ \\
\hline$C(31)$ & $-693(1)$ & $3350(1)$ & $599(1)$ & $21(1)$ \\
\hline$C(32)$ & $-1266(1)$ & $4137(1)$ & 381 (1) & $28(1)$ \\
\hline$C(33)$ & $-1981(2)$ & $3880(2)$ & $-494(1)$ & $35(1)$ \\
\hline$C(34)$ & $-2126(2)$ & $2846(2)$ & $-1163(1)$ & $38(1)$ \\
\hline$C(35)$ & $-1557(2)$ & $2062(2)$ & $-961(1)$ & $36(1)$ \\
\hline$C(36)$ & $-857(1)$ & $2308(1)$ & $-82(1)$ & $27(1)$ \\
\hline C (37) & $-2709(1)$ & $2902(1)$ & $1611(1)$ & $29(1)$ \\
\hline$C(38)$ & $-3739(1)$ & $2984(2)$ & $1915(1)$ & $33(1)$ \\
\hline$C(39)$ & $-3580(1)$ & $2757(1)$ & $2747(1)$ & $28(1)$ \\
\hline$C(40)$ & $-666(1)$ & $-62(1)$ & $2110(1)$ & $25(1)$ \\
\hline$C(41)$ & $-1297(2)$ & $-648(1)$ & $2461(1)$ & $31(1)$ \\
\hline$C(42)$ & $-1840(1)$ & $98(1)$ & $3128(1)$ & $29(1)$ \\
\hline$C(43)$ & $-4142(2)$ & $1787(2)$ & $4931(2)$ & $42(1)$ \\
\hline$C(44)$ & $-3522(2)$ & $2570(2)$ & $5734(2)$ & $44(1)$ \\
\hline$C(45)$ & $-2609(2)$ & $2798(2)$ & $5391(1)$ & $34(1)$ \\
\hline $\mathrm{B}(1)$ & $-1913(2)$ & $2192(2)$ & $3727(1)$ & $22(1)$ \\
\hline$N(1)$ & $-1959(1)$ & $2646(1)$ & $2216(1)$ & $22(1)$ \\
\hline
\end{tabular}




\begin{tabular}{|c|c|c|c|c|}
\hline $\mathrm{N}(2)$ & $-2513(1)$ & $2556(1)$ & $2922(1)$ & 21 (1) \\
\hline N (3) & $-1535(1)$ & $1065(1)$ & $3169(1)$ & $22(1)$ \\
\hline N (4) & $-806(1)$ & $979(1)$ & $2535(1)$ & $20(1)$ \\
\hline N (5) & $-2700(1)$ & $2174(1)$ & $4427(1)$ & 27 (1) \\
\hline $\mathrm{N}(6)$ & $-3663(1)$ & $1534(1)$ & $4132(1)$ & $36(1)$ \\
\hline$P(1)$ & $1521(1)$ & $1999(1)$ & $2668(1)$ & $17(1)$ \\
\hline$P(2)$ & $210(1)$ & $3583(1)$ & $1766(1)$ & $18(1)$ \\
\hline $\mathrm{Rh}(1)$ & $-236(1)$ & $2316(1)$ & $2303(1)$ & $17(1)$ \\
\hline$C(46)$ & $4581(4)$ & $1836(6)$ & $7750(5)$ & $57(2)$ \\
\hline C ( 47) & $4666(4)$ & $2867(7)$ & $8438(3)$ & $58(2)$ \\
\hline C ( 48) & $4245(5)$ & $3681(4)$ & $8176(4)$ & 104 (3) \\
\hline C (49) & $3739(5)$ & $3464(3)$ & $7225(4)$ & $85(2)$ \\
\hline$C(50)$ & $3654(5)$ & $2433(4)$ & $6537(2)$ & $64(2)$ \\
\hline C (51) & $4075(5)$ & $1619(3)$ & $6800(4)$ & $63(2)$ \\
\hline C (52) & $4968(5)$ & $955(5)$ & $8049(6)$ & $86(2)$ \\
\hline$C(46 B)$ & $4829(7)$ & $2093(9)$ & $8170(7)$ & $67(4)$ \\
\hline$C(47 B)$ & $4660(11)$ & $3161(8)$ & $8402(7)$ & $97(6)$ \\
\hline$C(48 B)$ & $4092(10)$ & $3494(10)$ & $7706(14)$ & $110(6)$ \\
\hline$C(49 B)$ & $3693(10)$ & $2759(18)$ & $6778(11)$ & $187(14)$ \\
\hline$C(50 B)$ & $3863(11)$ & 1691 (15) & $6547(5)$ & $168(12)$ \\
\hline$C(51 B)$ & $4430(9)$ & $1357(7)$ & $7243(10)$ & $91(5)$ \\
\hline$C(52 B)$ & $5347(8)$ & $1717(16)$ & $8879(11)$ & $138(7)$ \\
\hline
\end{tabular}


Table S9. Bond lengths [A] and angles [deg] for complex 3.

\begin{tabular}{|c|c|}
\hline$C(1)-C(6)$ & $1.396(2)$ \\
\hline$C(1)-C(2)$ & $1.400(2)$ \\
\hline$C(1)-P(1)$ & $1.8521(15)$ \\
\hline$C(2)-C(3)$ & $1.385(2)$ \\
\hline $\mathrm{C}(2)-\mathrm{H}(2)$ & 0.9500 \\
\hline$C(3)-C(4)$ & $1.393(2)$ \\
\hline $\mathrm{C}(3)-\mathrm{H}(3)$ & 0.9500 \\
\hline$C(4)-C(5)$ & $1.385(3)$ \\
\hline $\mathrm{C}(4)-\mathrm{H}(4)$ & 0.9500 \\
\hline$C(5)-C(6)$ & $1.395(2)$ \\
\hline C (5) -H (5) & 0.9500 \\
\hline$C(6)-H(6)$ & 0.9500 \\
\hline$C(7)-C(12)$ & $1.398(2)$ \\
\hline$C(7)-C(8)$ & $1.400(2)$ \\
\hline$C(7)-P(1)$ & $1.8422(16)$ \\
\hline$C(8)-C(9)$ & $1.394(2)$ \\
\hline $\mathrm{C}(8)-\mathrm{H}(8)$ & 0.9500 \\
\hline$C(9)-C(10)$ & $1.386(3)$ \\
\hline $\mathrm{C}(9)-\mathrm{H}(9)$ & 0.9500 \\
\hline$C(10)-C(11)$ & $1.388(3)$ \\
\hline $\mathrm{C}(10)-\mathrm{H}(10)$ & 0.9500 \\
\hline $\mathrm{C}(11)-\mathrm{C}(12)$ & $1.389(2)$ \\
\hline $\mathrm{C}(11)-\mathrm{H}(11)$ & 0.9500 \\
\hline $\mathrm{C}(12)-\mathrm{H}(12)$ & 0.9500 \\
\hline C (13) - C (14) & $1.394(2)$ \\
\hline$C(13)-C(18)$ & $1.401(2)$ \\
\hline$C(13)-P(1)$ & $1.8397(16)$ \\
\hline$C(14)-C(15)$ & $1.394(3)$ \\
\hline $\mathrm{C}(14)-\mathrm{H}(14)$ & 0.9500 \\
\hline$C(15)-C(16)$ & $1.386(3)$ \\
\hline$C(15)-H(15)$ & 0.9500 \\
\hline$C(16)-C(17)$ & 1.385 (3) \\
\hline $\mathrm{C}(16)-\mathrm{H}(16)$ & 0.9500 \\
\hline C (17) -C (18) & $1.393(2)$ \\
\hline $\mathrm{C}(17)-\mathrm{H}(17)$ & 0.9500 \\
\hline $\mathrm{C}(18)-\mathrm{H}(18)$ & 0.9500 \\
\hline$C(19)-C(24)$ & $1.398(2)$ \\
\hline$C(19)-C(20)$ & $1.400(2)$ \\
\hline$C(19)-P(2)$ & $1.8553(16)$ \\
\hline$C(20)-C(21)$ & $1.395(2)$ \\
\hline $\mathrm{C}(20)-\mathrm{H}(20)$ & 0.9500 \\
\hline$C(21)-C(22)$ & $1.385(3)$ \\
\hline $\mathrm{C}(21)-\mathrm{H}(21)$ & 0.9500 \\
\hline$C(22)-C(23)$ & $1.380(3)$ \\
\hline $\mathrm{C}(22)-\mathrm{H}(22)$ & 0.9500 \\
\hline$C(23)-C(24)$ & $1.393(2)$ \\
\hline $\mathrm{C}(23)-\mathrm{H}(23)$ & 0.9500 \\
\hline $\mathrm{C}(24)-\mathrm{H}(24)$ & 0.9500 \\
\hline$C(25)-C(26)$ & $1.395(2)$ \\
\hline$C(25)-C(30)$ & $1.399(2)$ \\
\hline$C(25)-P(2)$ & $1.8414(16)$ \\
\hline$C(26)-C(27)$ & $1.395(3)$ \\
\hline $\mathrm{C}(26)-\mathrm{H}(26)$ & 0.9500 \\
\hline C (27) - C (28) & $1.383(3)$ \\
\hline
\end{tabular}




\begin{tabular}{|c|c|}
\hline $\mathrm{C}(27)-\mathrm{H}(27)$ & 0.9500 \\
\hline$C(28)-C(29)$ & $1.381(3)$ \\
\hline $\mathrm{C}(28)-\mathrm{H}(28)$ & 0.9500 \\
\hline$C(29)-C(30)$ & $1.390(2)$ \\
\hline $\mathrm{C}(29)-\mathrm{H}(29)$ & 0.9500 \\
\hline $\mathrm{C}(30)-\mathrm{H}(30)$ & 0.9500 \\
\hline$C(31)-C(36)$ & $1.397(2)$ \\
\hline$C(31)-C(32)$ & $1.400(2)$ \\
\hline$C(31)-P(2)$ & $1.8474(16)$ \\
\hline$C(32)-C(33)$ & $1.390(2)$ \\
\hline $\mathrm{C}(32)-\mathrm{H}(32)$ & 0.9500 \\
\hline$C(33)-C(34)$ & $1.382(3)$ \\
\hline $\mathrm{C}(33)-\mathrm{H}(33)$ & 0.9500 \\
\hline$C(34)-C(35)$ & $1.383(3)$ \\
\hline $\mathrm{C}(34)-\mathrm{H}(34)$ & 0.9500 \\
\hline$C(35)-C(36)$ & $1.389(2)$ \\
\hline $\mathrm{C}(35)-\mathrm{H}(35)$ & 0.9500 \\
\hline $\mathrm{C}(36)-\mathrm{H}(36)$ & 0.9500 \\
\hline $\mathrm{C}(37)-\mathrm{N}(1)$ & $1.340(2)$ \\
\hline$C(37)-C(38)$ & $1.392(2)$ \\
\hline $\mathrm{C}(37)-\mathrm{H}(37)$ & 0.9500 \\
\hline$C(38)-C(39)$ & $1.381(2)$ \\
\hline $\mathrm{C}(38)-\mathrm{H}(38)$ & 0.9500 \\
\hline $\mathrm{C}(39)-\mathrm{N}(2)$ & $1.347(2)$ \\
\hline $\mathrm{C}(39)-\mathrm{H}(39)$ & 0.9500 \\
\hline $\mathrm{C}(40)-\mathrm{N}(4)$ & $1.343(2)$ \\
\hline$C(40)-C(41)$ & $1.390(2)$ \\
\hline $\mathrm{C}(40)-\mathrm{H}(40)$ & 0.9500 \\
\hline$C(41)-C(42)$ & $1.381(3)$ \\
\hline $\mathrm{C}(41)-\mathrm{H}(41)$ & 0.9500 \\
\hline $\mathrm{C}(42)-\mathrm{N}(3)$ & $1.346(2)$ \\
\hline $\mathrm{C}(42)-\mathrm{H}(42)$ & 0.9500 \\
\hline$C(43)-N(6)$ & $1.335(2)$ \\
\hline$C(43)-C(44)$ & $1.391(3)$ \\
\hline $\mathrm{C}(43)-\mathrm{H}(43)$ & 0.9500 \\
\hline$C(44)-C(45)$ & $1.374(3)$ \\
\hline $\mathrm{C}(44)-\mathrm{H}(44)$ & 0.9500 \\
\hline $\mathrm{C}(45)-\mathrm{N}(5)$ & $1.361(2)$ \\
\hline $\mathrm{C}(45)-\mathrm{H}(45)$ & 0.9500 \\
\hline$B(1)-N(5)$ & $1.526(2)$ \\
\hline $\mathrm{B}(1)-\mathrm{N}(3)$ & $1.558(2)$ \\
\hline$B(1)-N(2)$ & $1.564(2)$ \\
\hline $\mathrm{B}(1)-\mathrm{H}(1)$ & 1.126 (15) \\
\hline$N(1)-N(2)$ & $1.3741(18)$ \\
\hline $\mathrm{N}(1)-\mathrm{Rh}(1)$ & $2.1575(13)$ \\
\hline$N(3)-N(4)$ & $1.3661(18)$ \\
\hline $\mathrm{N}(4)-\mathrm{Rh}(1)$ & $2.1111(13)$ \\
\hline$N(5)-N(6)$ & $1.378(2)$ \\
\hline $\mathrm{P}(1)-\mathrm{Rh}(1)$ & $2.2299(4)$ \\
\hline $\mathrm{P}(2)-\mathrm{Rh}(1)$ & $2.2558(4)$ \\
\hline$C(46)-C(47)$ & 1.3900 \\
\hline$C(46)-C(51)$ & 1.3900 \\
\hline$C(46)-C(52)$ & $1.489(7)$ \\
\hline$C(47)-C(48)$ & 1.3900 \\
\hline $\mathrm{C}(47)-\mathrm{H}(47)$ & 0.9500 \\
\hline$C(48)-C(49)$ & 1.3900 \\
\hline $\mathrm{C}(48)-\mathrm{H}(48)$ & 0.9500 \\
\hline
\end{tabular}




\begin{tabular}{|c|c|}
\hline$C(49)-C(50)$ & 1.3900 \\
\hline $\mathrm{C}(49)-\mathrm{H}(49)$ & 0.9500 \\
\hline$C(50)-C(51)$ & 1.3900 \\
\hline $\mathrm{C}(50)-\mathrm{H}(50)$ & 0.9500 \\
\hline $\mathrm{C}(51)-\mathrm{H}(51)$ & 0.9500 \\
\hline $\mathrm{C}(52)-\mathrm{H}(52 \mathrm{~A})$ & 0.9800 \\
\hline $\mathrm{C}(52)-\mathrm{H}(52 \mathrm{~B})$ & 0.9800 \\
\hline $\mathrm{C}(52)-\mathrm{H}(52 \mathrm{C})$ & 0.9800 \\
\hline$C(46 B)-C(47 B)$ & 1.3900 \\
\hline$C(46 B)-C(51 B)$ & 1.3900 \\
\hline$C(46 B)-C(52 B)$ & $1.419(11)$ \\
\hline$C(47 B)-C(48 B)$ & 1.3900 \\
\hline$C(47 B)-H(47 B)$ & 0.9500 \\
\hline$C(48 B)-C(49 B)$ & 1.3900 \\
\hline$C(48 B)-H(48 B)$ & 0.9500 \\
\hline$C(49 B)-C(50 B)$ & 1.3900 \\
\hline$C(49 B)-H(49 B)$ & 0.9500 \\
\hline$C(50 B)-C(51 B)$ & 1.3900 \\
\hline $\mathrm{C}(50 \mathrm{~B})-\mathrm{H}(50 \mathrm{~B})$ & 0.9500 \\
\hline$C(51 B)-H(51 B)$ & 0.9500 \\
\hline$C(52 B)-H(52 D)$ & 0.9800 \\
\hline$C(52 B)-H(52 E)$ & 0.9800 \\
\hline$C(52 B)-H(52 F)$ & 0.9800 \\
\hline$C(6)-C(1)-C(2)$ & $118.85(14)$ \\
\hline$C(6)-C(1)-P(1)$ & $122.64(12)$ \\
\hline$C(2)-C(1)-P(1)$ & $118.33(11)$ \\
\hline$C(3)-C(2)-C(1)$ & $120.51(15)$ \\
\hline $\mathrm{C}(3)-\mathrm{C}(2)-\mathrm{H}(2)$ & 119.7 \\
\hline $\mathrm{C}(1)-\mathrm{C}(2)-\mathrm{H}(2)$ & 119.7 \\
\hline$C(2)-C(3)-C(4)$ & $120.31(16)$ \\
\hline $\mathrm{C}(2)-\mathrm{C}(3)-\mathrm{H}(3)$ & 119.8 \\
\hline $\mathrm{C}(4)-\mathrm{C}(3)-\mathrm{H}(3)$ & 119.8 \\
\hline$C(5)-C(4)-C(3)$ & $119.64(16)$ \\
\hline $\mathrm{C}(5)-\mathrm{C}(4)-\mathrm{H}(4)$ & 120.2 \\
\hline $\mathrm{C}(3)-\mathrm{C}(4)-\mathrm{H}(4)$ & 120.2 \\
\hline$C(4)-C(5)-C(6)$ & $120.28(16)$ \\
\hline $\mathrm{C}(4)-\mathrm{C}(5)-\mathrm{H}(5)$ & 119.9 \\
\hline$C(6)-C(5)-H(5)$ & 119.9 \\
\hline$C(5)-C(6)-C(1)$ & $120.35(16)$ \\
\hline$C(5)-C(6)-H(6)$ & 119.8 \\
\hline $\mathrm{C}(1)-\mathrm{C}(6)-\mathrm{H}(6)$ & 119.8 \\
\hline$C(12)-C(7)-C(8)$ & $118.52(15)$ \\
\hline$C(12)-C(7)-P(1)$ & $122.51(12)$ \\
\hline$C(8)-C(7)-P(1)$ & $118.26(12)$ \\
\hline$C(9)-C(8)-C(7)$ & $120.50(16)$ \\
\hline $\mathrm{C}(9)-\mathrm{C}(8)-\mathrm{H}(8)$ & 119.8 \\
\hline $\mathrm{C}(7)-\mathrm{C}(8)-\mathrm{H}(8)$ & 119.8 \\
\hline$C(10)-C(9)-C(8)$ & $120.09(16)$ \\
\hline $\mathrm{C}(10)-\mathrm{C}(9)-\mathrm{H}(9)$ & 120.0 \\
\hline $\mathrm{C}(8)-\mathrm{C}(9)-\mathrm{H}(9)$ & 120.0 \\
\hline$C(9)-C(10)-C(11)$ & $120.04(16)$ \\
\hline $\mathrm{C}(9)-\mathrm{C}(10)-\mathrm{H}(10)$ & 120.0 \\
\hline $\mathrm{C}(11)-\mathrm{C}(10)-\mathrm{H}(10)$ & 120.0 \\
\hline$C(10)-C(11)-C(12)$ & $119.97(17)$ \\
\hline $\mathrm{C}(10)-\mathrm{C}(11)-\mathrm{H}(11)$ & 120.0 \\
\hline $\mathrm{C}(12)-\mathrm{C}(11)-\mathrm{H}(11)$ & 120.0 \\
\hline
\end{tabular}




\begin{tabular}{|c|c|}
\hline$C(11)-C(12)-C(7)$ & $120.83(16)$ \\
\hline $\mathrm{C}(11)-\mathrm{C}(12)-\mathrm{H}(12)$ & 119.6 \\
\hline $\mathrm{C}(7)-\mathrm{C}(12)-\mathrm{H}(12)$ & 119.6 \\
\hline$C(14)-C(13)-C(18)$ & $118.04(15)$ \\
\hline$C(14)-C(13)-P(1)$ & $127.13(13)$ \\
\hline $\mathrm{C}(18)-\mathrm{C}(13)-\mathrm{P}(1)$ & $114.76(12)$ \\
\hline$C(13)-C(14)-C(15)$ & $120.52(17)$ \\
\hline $\mathrm{C}(13)-\mathrm{C}(14)-\mathrm{H}(14)$ & 119.7 \\
\hline $\mathrm{C}(15)-\mathrm{C}(14)-\mathrm{H}(14)$ & 119.7 \\
\hline$C(16)-C(15)-C(14)$ & $120.75(17)$ \\
\hline $\mathrm{C}(16)-\mathrm{C}(15)-\mathrm{H}(15)$ & 119.6 \\
\hline $\mathrm{C}(14)-\mathrm{C}(15)-\mathrm{H}(15)$ & 119.6 \\
\hline$C(17)-C(16)-C(15)$ & $119.55(17)$ \\
\hline $\mathrm{C}(17)-\mathrm{C}(16)-\mathrm{H}(16)$ & 120.2 \\
\hline $\mathrm{C}(15)-\mathrm{C}(16)-\mathrm{H}(16)$ & 120.2 \\
\hline$C(16)-C(17)-C(18)$ & $119.76(17)$ \\
\hline $\mathrm{C}(16)-\mathrm{C}(17)-\mathrm{H}(17)$ & 120.1 \\
\hline $\mathrm{C}(18)-\mathrm{C}(17)-\mathrm{H}(17)$ & 120.1 \\
\hline$C(17)-C(18)-C(13)$ & $121.33(16)$ \\
\hline $\mathrm{C}(17)-\mathrm{C}(18)-\mathrm{H}(18)$ & 119.3 \\
\hline $\mathrm{C}(13)-\mathrm{C}(18)-\mathrm{H}(18)$ & 119.3 \\
\hline$C(24)-C(19)-C(20)$ & $118.32(15)$ \\
\hline$C(24)-C(19)-P(2)$ & $121.13(13)$ \\
\hline$C(20)-C(19)-P(2)$ & $120.55(12)$ \\
\hline$C(21)-C(20)-C(19)$ & $120.62(16)$ \\
\hline $\mathrm{C}(21)-\mathrm{C}(20)-\mathrm{H}(20)$ & 119.7 \\
\hline $\mathrm{C}(19)-\mathrm{C}(20)-\mathrm{H}(20)$ & 119.7 \\
\hline$C(22)-C(21)-C(20)$ & $119.96(17)$ \\
\hline $\mathrm{C}(22)-\mathrm{C}(21)-\mathrm{H}(21)$ & 120.0 \\
\hline $\mathrm{C}(20)-\mathrm{C}(21)-\mathrm{H}(21)$ & 120.0 \\
\hline$C(23)-C(22)-C(21)$ & $120.24(17)$ \\
\hline $\mathrm{C}(23)-\mathrm{C}(22)-\mathrm{H}(22)$ & 119.9 \\
\hline $\mathrm{C}(21)-\mathrm{C}(22)-\mathrm{H}(22)$ & 119.9 \\
\hline$C(22)-C(23)-C(24)$ & $119.98(17)$ \\
\hline $\mathrm{C}(22)-\mathrm{C}(23)-\mathrm{H}(23)$ & 120.0 \\
\hline $\mathrm{C}(24)-\mathrm{C}(23)-\mathrm{H}(23)$ & 120.0 \\
\hline$C(23)-C(24)-C(19)$ & $120.85(17)$ \\
\hline $\mathrm{C}(23)-\mathrm{C}(24)-\mathrm{H}(24)$ & 119.6 \\
\hline $\mathrm{C}(19)-\mathrm{C}(24)-\mathrm{H}(24)$ & 119.6 \\
\hline$C(26)-C(25)-C(30)$ & $118.84(15)$ \\
\hline$C(26)-C(25)-P(2)$ & $119.59(13)$ \\
\hline$C(30)-C(25)-P(2)$ & $121.47(13)$ \\
\hline$C(27)-C(26)-C(25)$ & $119.95(17)$ \\
\hline $\mathrm{C}(27)-\mathrm{C}(26)-\mathrm{H}(26)$ & 120.0 \\
\hline $\mathrm{C}(25)-\mathrm{C}(26)-\mathrm{H}(26)$ & 120.0 \\
\hline$C(28)-C(27)-C(26)$ & $120.36(18)$ \\
\hline $\mathrm{C}(28)-\mathrm{C}(27)-\mathrm{H}(27)$ & 119.8 \\
\hline $\mathrm{C}(26)-\mathrm{C}(27)-\mathrm{H}(27)$ & 119.8 \\
\hline$C(29)-C(28)-C(27)$ & $120.32(17)$ \\
\hline $\mathrm{C}(29)-\mathrm{C}(28)-\mathrm{H}(28)$ & 119.8 \\
\hline $\mathrm{C}(27)-\mathrm{C}(28)-\mathrm{H}(28)$ & 119.8 \\
\hline$C(28)-C(29)-C(30)$ & $119.67(17)$ \\
\hline $\mathrm{C}(28)-\mathrm{C}(29)-\mathrm{H}(29)$ & 120.2 \\
\hline $\mathrm{C}(30)-\mathrm{C}(29)-\mathrm{H}(29)$ & 120.2 \\
\hline$C(29)-C(30)-C(25)$ & $120.86(16)$ \\
\hline $\mathrm{C}(29)-\mathrm{C}(30)-\mathrm{H}(30)$ & 119.6 \\
\hline $\mathrm{C}(25)-\mathrm{C}(30)-\mathrm{H}(30)$ & 119.6 \\
\hline
\end{tabular}




\begin{tabular}{|c|c|}
\hline$C(36)-C(31)-C(32)$ & $118.38(15)$ \\
\hline$C(36)-C(31)-P(2)$ & $117.33(12)$ \\
\hline$C(32)-C(31)-P(2)$ & $124.19(13)$ \\
\hline$C(33)-C(32)-C(31)$ & $120.39(17)$ \\
\hline $\mathrm{C}(33)-\mathrm{C}(32)-\mathrm{H}(32)$ & 119.8 \\
\hline $\mathrm{C}(31)-\mathrm{C}(32)-\mathrm{H}(32)$ & 119.8 \\
\hline$C(34)-C(33)-C(32)$ & $120.44(16)$ \\
\hline $\mathrm{C}(34)-\mathrm{C}(33)-\mathrm{H}(33)$ & 119.8 \\
\hline $\mathrm{C}(32)-\mathrm{C}(33)-\mathrm{H}(33)$ & 119.8 \\
\hline$C(33)-C(34)-C(35)$ & $119.82(17)$ \\
\hline $\mathrm{C}(33)-\mathrm{C}(34)-\mathrm{H}(34)$ & 120.1 \\
\hline $\mathrm{C}(35)-\mathrm{C}(34)-\mathrm{H}(34)$ & 120.1 \\
\hline$C(34)-C(35)-C(36)$ & $120.12(18)$ \\
\hline $\mathrm{C}(34)-\mathrm{C}(35)-\mathrm{H}(35)$ & 119.9 \\
\hline $\mathrm{C}(36)-\mathrm{C}(35)-\mathrm{H}(35)$ & 119.9 \\
\hline$C(35)-C(36)-C(31)$ & $120.83(16)$ \\
\hline $\mathrm{C}(35)-\mathrm{C}(36)-\mathrm{H}(36)$ & 119.6 \\
\hline $\mathrm{C}(31)-\mathrm{C}(36)-\mathrm{H}(36)$ & 119.6 \\
\hline $\mathrm{N}(1)-\mathrm{C}(37)-\mathrm{C}(38)$ & $110.89(15)$ \\
\hline $\mathrm{N}(1)-\mathrm{C}(37)-\mathrm{H}(37)$ & 124.6 \\
\hline $\mathrm{C}(38)-\mathrm{C}(37)-\mathrm{H}(37)$ & 124.6 \\
\hline $\mathrm{C}(39)-\mathrm{C}(38)-\mathrm{C}(37)$ & $104.87(15)$ \\
\hline $\mathrm{C}(39)-\mathrm{C}(38)-\mathrm{H}(38)$ & 127.6 \\
\hline $\mathrm{C}(37)-\mathrm{C}(38)-\mathrm{H}(38)$ & 127.6 \\
\hline $\mathrm{N}(2)-\mathrm{C}(39)-\mathrm{C}(38)$ & $108.56(15)$ \\
\hline $\mathrm{N}(2)-\mathrm{C}(39)-\mathrm{H}(39)$ & 125.7 \\
\hline $\mathrm{C}(38)-\mathrm{C}(39)-\mathrm{H}(39)$ & 125.7 \\
\hline $\mathrm{N}(4)-\mathrm{C}(40)-\mathrm{C}(41)$ & $110.92(15)$ \\
\hline $\mathrm{N}(4)-\mathrm{C}(40)-\mathrm{H}(40)$ & 124.5 \\
\hline $\mathrm{C}(41)-\mathrm{C}(40)-\mathrm{H}(40)$ & 124.5 \\
\hline$C(42)-C(41)-C(40)$ & $104.62(15)$ \\
\hline $\mathrm{C}(42)-\mathrm{C}(41)-\mathrm{H}(41)$ & 127.7 \\
\hline $\mathrm{C}(40)-\mathrm{C}(41)-\mathrm{H}(41)$ & 127.7 \\
\hline $\mathrm{N}(3)-\mathrm{C}(42)-\mathrm{C}(41)$ & $108.63(16)$ \\
\hline $\mathrm{N}(3)-\mathrm{C}(42)-\mathrm{H}(42)$ & 125.7 \\
\hline $\mathrm{C}(41)-\mathrm{C}(42)-\mathrm{H}(42)$ & 125.7 \\
\hline$N(6)-C(43)-C(44)$ & $112.14(19)$ \\
\hline$N(6)-C(43)-H(43)$ & 123.9 \\
\hline $\mathrm{C}(44)-\mathrm{C}(43)-\mathrm{H}(43)$ & 123.9 \\
\hline$C(45)-C(44)-C(43)$ & $104.74(18)$ \\
\hline $\mathrm{C}(45)-\mathrm{C}(44)-\mathrm{H}(44)$ & 127.6 \\
\hline $\mathrm{C}(43)-\mathrm{C}(44)-\mathrm{H}(44)$ & 127.6 \\
\hline$N(5)-C(45)-C(44)$ & $108.09(19)$ \\
\hline $\mathrm{N}(5)-\mathrm{C}(45)-\mathrm{H}(45)$ & 126.0 \\
\hline $\mathrm{C}(44)-\mathrm{C}(45)-\mathrm{H}(45)$ & 126.0 \\
\hline$N(5)-B(1)-N(3)$ & $111.70(14)$ \\
\hline $\mathrm{N}(5)-\mathrm{B}(1)-\mathrm{N}(2)$ & $110.16(13)$ \\
\hline $\mathrm{N}(3)-\mathrm{B}(1)-\mathrm{N}(2)$ & $105.52(13)$ \\
\hline $\mathrm{N}(5)-\mathrm{B}(1)-\mathrm{H}(1)$ & $109.6(8)$ \\
\hline $\mathrm{N}(3)-\mathrm{B}(1)-\mathrm{H}(1)$ & $110.5(8)$ \\
\hline $\mathrm{N}(2)-\mathrm{B}(1)-\mathrm{H}(1)$ & $109.3(8)$ \\
\hline $\mathrm{C}(37)-\mathrm{N}(1)-\mathrm{N}(2)$ & $105.97(13)$ \\
\hline $\mathrm{C}(37)-\mathrm{N}(1)-\mathrm{Rh}(1)$ & $136.79(11)$ \\
\hline $\mathrm{N}(2)-\mathrm{N}(1)-\mathrm{Rh}(1)$ & $117.20(9)$ \\
\hline $\mathrm{C}(39)-\mathrm{N}(2)-\mathrm{N}(1)$ & $109.72(13)$ \\
\hline $\mathrm{C}(39)-\mathrm{N}(2)-\mathrm{B}(1)$ & $129.64(14)$ \\
\hline $\mathrm{N}(1)-\mathrm{N}(2)-\mathrm{B}(1)$ & $120.52(12)$ \\
\hline
\end{tabular}




\begin{tabular}{|c|c|}
\hline $\mathrm{C}(42)-\mathrm{N}(3)-\mathrm{N}(4)$ & $109.90(13)$ \\
\hline $\mathrm{C}(42)-\mathrm{N}(3)-\mathrm{B}(1)$ & $132.53(14)$ \\
\hline$N(4)-N(3)-B(1)$ & $117.41(13)$ \\
\hline $\mathrm{C}(40)-\mathrm{N}(4)-\mathrm{N}(3)$ & $105.92(13)$ \\
\hline $\mathrm{C}(40)-\mathrm{N}(4)-\mathrm{Rh}(1)$ & $132.63(11)$ \\
\hline$N(3)-N(4)-R h(1)$ & $121.24(9)$ \\
\hline$C(45)-N(5)-N(6)$ & $110.12(15)$ \\
\hline $\mathrm{C}(45)-\mathrm{N}(5)-\mathrm{B}(1)$ & $126.85(16)$ \\
\hline $\mathrm{N}(6)-\mathrm{N}(5)-\mathrm{B}(1)$ & $122.94(14)$ \\
\hline $\mathrm{C}(43)-\mathrm{N}(6)-\mathrm{N}(5)$ & $104.91(16)$ \\
\hline$C(13)-P(1)-C(7)$ & $109.05(7)$ \\
\hline$C(13)-P(1)-C(1)$ & $101.25(7)$ \\
\hline$C(7)-P(1)-C(1)$ & $95.69(7)$ \\
\hline $\mathrm{C}(13)-\mathrm{P}(1)-\mathrm{Rh}(1)$ & $116.17(5)$ \\
\hline $\mathrm{C}(7)-\mathrm{P}(1)-\mathrm{Rh}(1)$ & $116.23(5)$ \\
\hline $\mathrm{C}(1)-\mathrm{P}(1)-\mathrm{Rh}(1)$ & $115.72(5)$ \\
\hline$C(25)-P(2)-C(31)$ & $102.16(7)$ \\
\hline$C(25)-P(2)-C(19)$ & $100.96(7)$ \\
\hline$C(31)-P(2)-C(19)$ & $100.48(7)$ \\
\hline $\mathrm{C}(25)-\mathrm{P}(2)-\mathrm{Rh}(1)$ & $119.22(6)$ \\
\hline $\mathrm{C}(31)-\mathrm{P}(2)-\mathrm{Rh}(1)$ & $108.34(5)$ \\
\hline $\mathrm{C}(19)-\mathrm{P}(2)-\mathrm{Rh}(1)$ & $122.50(5)$ \\
\hline $\mathrm{N}(4)-\mathrm{Rh}(1)-\mathrm{N}(1)$ & $83.37(5)$ \\
\hline$N(4)-\operatorname{Rh}(1)-P(1)$ & $91.52(3)$ \\
\hline$N(1)-\operatorname{Rh}(1)-P(1)$ & $169.58(4)$ \\
\hline$N(4)-\operatorname{Rh}(1)-P(2)$ & $169.18(4)$ \\
\hline $\mathrm{N}(1)-\mathrm{Rh}(1)-\mathrm{P}(2)$ & $92.98(4)$ \\
\hline $\mathrm{P}(1)-\mathrm{Rh}(1)-\mathrm{P}(2)$ & $93.605(15)$ \\
\hline$C(47)-C(46)-C(51)$ & 120.0 \\
\hline$C(47)-C(46)-C(52)$ & $119.8(6)$ \\
\hline$C(51)-C(46)-C(52)$ & $120.0(6)$ \\
\hline$C(48)-C(47)-C(46)$ & 120.0 \\
\hline $\mathrm{C}(48)-\mathrm{C}(47)-\mathrm{H}(47)$ & 120.0 \\
\hline $\mathrm{C}(46)-\mathrm{C}(47)-\mathrm{H}(47)$ & 120.0 \\
\hline$C(47)-C(48)-C(49)$ & 120.0 \\
\hline $\mathrm{C}(47)-\mathrm{C}(48)-\mathrm{H}(48)$ & 120.0 \\
\hline $\mathrm{C}(49)-\mathrm{C}(48)-\mathrm{H}(48)$ & 120.0 \\
\hline$C(48)-C(49)-C(50)$ & 120.0 \\
\hline $\mathrm{C}(48)-\mathrm{C}(49)-\mathrm{H}(49)$ & 120.0 \\
\hline $\mathrm{C}(50)-\mathrm{C}(49)-\mathrm{H}(49)$ & 120.0 \\
\hline$C(51)-C(50)-C(49)$ & 120.0 \\
\hline $\mathrm{C}(51)-\mathrm{C}(50)-\mathrm{H}(50)$ & 120.0 \\
\hline $\mathrm{C}(49)-\mathrm{C}(50)-\mathrm{H}(50)$ & 120.0 \\
\hline$C(50)-C(51)-C(46)$ & 120.0 \\
\hline $\mathrm{C}(50)-\mathrm{C}(51)-\mathrm{H}(51)$ & 120.0 \\
\hline $\mathrm{C}(46)-\mathrm{C}(51)-\mathrm{H}(51)$ & 120.0 \\
\hline$C(46)-C(52)-H(52 A)$ & 109.5 \\
\hline$C(46)-C(52)-H(52 B)$ & 109.5 \\
\hline $\mathrm{H}(52 \mathrm{~A})-\mathrm{C}(52)-\mathrm{H}(52 \mathrm{~B})$ & 109.5 \\
\hline $\mathrm{C}(46)-\mathrm{C}(52)-\mathrm{H}(52 \mathrm{C})$ & 109.5 \\
\hline $\mathrm{H}(52 \mathrm{~A})-\mathrm{C}(52)-\mathrm{H}(52 \mathrm{C})$ & 109.5 \\
\hline $\mathrm{H}(52 \mathrm{~B})-\mathrm{C}(52)-\mathrm{H}(52 \mathrm{C})$ & 109.5 \\
\hline$C(47 B)-C(46 B)-C(51 B)$ & 120.0 \\
\hline$C(47 B)-C(46 B)-C(52 B)$ & $121.5(13)$ \\
\hline$C(51 B)-C(46 B)-C(52 B)$ & $118.4(13)$ \\
\hline$C(48 B)-C(47 B)-C(46 B)$ & 120.0 \\
\hline$C(48 B)-C(47 B)-H(47 B)$ & 120.0 \\
\hline
\end{tabular}




$$
\begin{array}{ll}
\text { C (46B)-C (47B)-H (47B) } & 120.0 \\
\text { C (49B)-C (48B)-C (47B) } & 120.0 \\
\text { C (49B)-C (48B)-H (48B) } & 120.0 \\
\text { C (47B)-C (48B)-H (48B) } & 120.0 \\
\text { C (48B)-C (49B)-C (50B) } & 120.0 \\
\text { C (48B)-C (49B)-H (49B) } & 120.0 \\
\text { C (50B)-C (49B)-H (49B) } & 120.0 \\
\text { C (51B)-C (50B)-C (49B) } & 120.0 \\
\text { C (51B)-C (50B)-H (50B) } & 120.0 \\
\text { C (49B)-C (50B)-H (50B) } & 120.0 \\
\text { C (50B)-C (51B)-C (46B) } & 120.0 \\
\text { C (50B)-C (51B)-H (51B) } & 120.0 \\
\text { C (46B)-C (51B)-H (51B) } & 120.0 \\
\text { C (46B)-C (52B)-H (52D) } & 109.5 \\
\text { C (46B)-C (52B)-H (52E) } & 109.5 \\
\text { H (52D)-C (52B)-H (52E) } & 109.5 \\
\text { C (46B)-C (52B)-H (52F) } & 109.5 \\
\text { H (52D)-C (52B)-H (52F) } & 109.5 \\
\text { H (52E)-C (52B)-H (52F) } & 109.5
\end{array}
$$

Symmetry transformations used to generate equivalent atoms: 
Table S10. Anisotropic displacement parameters ( $A^{\wedge} 2 \times 10^{\wedge} 3$ ) for complex 3. The anisotropic displacement factor exponent takes the form:

$-2 \mathrm{pi}^{\wedge} 2\left[\mathrm{~h}^{\wedge} 2 \mathrm{a}^{\star \wedge} 2 \mathrm{U11}+\ldots+2 \mathrm{~h} \mathrm{k} \mathrm{a}^{\star} \mathrm{b}^{\star} \mathrm{U} 12\right.$ ]

\begin{tabular}{|c|c|c|c|c|c|c|}
\hline & $\mathrm{U} 11$ & $\mathrm{U} 22$ & U33 & $\mathrm{U} 23$ & $\mathrm{U} 13$ & $\mathrm{U} 12$ \\
\hline$C(1)$ & $20(1)$ & $16(1)$ & $19(1)$ & $8(1)$ & $-1(1)$ & $1(1)$ \\
\hline$C(2)$ & $25(1)$ & $21(1)$ & $23(1)$ & $9(1)$ & $3(1)$ & $5(1)$ \\
\hline$C(3)$ & $38(1)$ & $28(1)$ & $24(1)$ & $13(1)$ & $7(1)$ & $2(1)$ \\
\hline$C(4)$ & $54(1)$ & $25(1)$ & $29(1)$ & $17(1)$ & $6(1)$ & $11(1)$ \\
\hline$C(5)$ & $46(1)$ & $27(1)$ & $31(1)$ & $14(1)$ & $8(1)$ & $18(1)$ \\
\hline$C(6)$ & $29(1)$ & $24(1)$ & $24(1)$ & $10(1)$ & $5(1)$ & $7(1)$ \\
\hline$C(7)$ & $23(1)$ & $19(1)$ & $22(1)$ & $12(1)$ & $3(1)$ & $1(1)$ \\
\hline$C(8)$ & $28(1)$ & $23(1)$ & $24(1)$ & $14(1)$ & $5(1)$ & $3(1)$ \\
\hline$C(9)$ & $44(1)$ & $20(1)$ & $28(1)$ & $10(1)$ & $10(1)$ & $1(1)$ \\
\hline$C(10)$ & $44(1)$ & $26(1)$ & $32(1)$ & $9(1)$ & $-2(1)$ & $-11(1)$ \\
\hline$C(11)$ & $29(1)$ & $33(1)$ & $40(1)$ & $16(1)$ & $-7(1)$ & $-5(1)$ \\
\hline$C(12)$ & $25(1)$ & $21(1)$ & $33(1)$ & $12(1)$ & $1(1)$ & $1(1)$ \\
\hline$C(13)$ & $23(1)$ & $20(1)$ & $23(1)$ & $13(1)$ & $7(1)$ & $8(1)$ \\
\hline$C(14)$ & $24(1)$ & $28(1)$ & $32(1)$ & $13(1)$ & $8(1)$ & $4(1)$ \\
\hline$C(15)$ & $30(1)$ & $42(1)$ & $47(1)$ & $19(1)$ & $20(1)$ & $9(1)$ \\
\hline$C(16)$ & $47(1)$ & $38(1)$ & $34(1)$ & $14(1)$ & $22(1)$ & $16(1)$ \\
\hline$C(17)$ & $45(1)$ & $28(1)$ & $23(1)$ & $8(1)$ & $7(1)$ & $4(1)$ \\
\hline$C(18)$ & $27(1)$ & $28(1)$ & $23(1)$ & $13(1)$ & $5(1)$ & $4(1)$ \\
\hline$C(19)$ & $22(1)$ & $20(1)$ & $27(1)$ & $15(1)$ & $8(1)$ & $6(1)$ \\
\hline$C(20)$ & $26(1)$ & $28(1)$ & $29(1)$ & $18(1)$ & $7(1)$ & $5(1)$ \\
\hline$C(21)$ & $22(1)$ & $38(1)$ & $43(1)$ & $25(1)$ & $1(1)$ & $1(1)$ \\
\hline$C(22)$ & $24(1)$ & $44(1)$ & $48(1)$ & $30(1)$ & $15(1)$ & $9(1)$ \\
\hline C (23) & $33(1)$ & $39(1)$ & $35(1)$ & $18(1)$ & $17(1)$ & $11(1)$ \\
\hline$C(24)$ & $27(1)$ & $28(1)$ & $32(1)$ & $13(1)$ & $8(1)$ & $5(1)$ \\
\hline$C(25)$ & $23(1)$ & $21(1)$ & $24(1)$ & $11(1)$ & $4(1)$ & $6(1)$ \\
\hline C (26) & $34(1)$ & $27(1)$ & $31(1)$ & $16(1)$ & $12(1)$ & $7(1)$ \\
\hline$C(27)$ & $55(1)$ & $34(1)$ & $39(1)$ & $14(1)$ & $28(1)$ & $14(1)$ \\
\hline$C(28)$ & $60(1)$ & $23(1)$ & $43(1)$ & $9(1)$ & $21(1)$ & $13(1)$ \\
\hline C (29) & $45(1)$ & $21(1)$ & $41(1)$ & $15(1)$ & $11(1)$ & $5(1)$ \\
\hline$C(30)$ & $31(1)$ & $25(1)$ & $28(1)$ & $13(1)$ & $10(1)$ & $6(1)$ \\
\hline$C(31)$ & $21(1)$ & $25(1)$ & $22(1)$ & $14(1)$ & $6(1)$ & $2(1)$ \\
\hline$C(32)$ & $30(1)$ & $28(1)$ & $30(1)$ & $17(1)$ & $5(1)$ & $5(1)$ \\
\hline$C(33)$ & $36(1)$ & $41(1)$ & $39(1)$ & $28(1)$ & $2(1)$ & $8(1)$ \\
\hline C (34) & $39(1)$ & $49(1)$ & $30(1)$ & $22(1)$ & $-6(1)$ & $-3(1)$ \\
\hline$C(35)$ & $41(1)$ & $34(1)$ & $29(1)$ & $10(1)$ & $-1(1)$ & $-1(1)$ \\
\hline$C(36)$ & $28(1)$ & $27(1)$ & $28(1)$ & $13(1)$ & $3(1)$ & $4(1)$ \\
\hline C (37) & $22(1)$ & $38(1)$ & $36(1)$ & $25(1)$ & $4(1)$ & $5(1)$ \\
\hline $\mathrm{C}(38)$ & $19(1)$ & $46(1)$ & $45(1)$ & $30(1)$ & $3(1)$ & $7(1)$ \\
\hline C (39) & $19(1)$ & $31(1)$ & $36(1)$ & $15(1)$ & $9(1)$ & $5(1)$ \\
\hline$C(40)$ & $27(1)$ & $22(1)$ & $28(1)$ & $10(1)$ & $5(1)$ & $5(1)$ \\
\hline$C(41)$ & $37(1)$ & $20(1)$ & $39(1)$ & $14(1)$ & $6(1)$ & $2(1)$ \\
\hline$C(42)$ & $29(1)$ & $29(1)$ & $37(1)$ & $21(1)$ & $7(1)$ & $-1(1)$ \\
\hline$C(43)$ & $37(1)$ & $54(1)$ & $58(1)$ & $40(1)$ & $26(1)$ & $21(1)$ \\
\hline$C(44)$ & $60(1)$ & $47(1)$ & $42(1)$ & $30(1)$ & $32(1)$ & $33(1)$ \\
\hline$C(45)$ & $49(1)$ & $30(1)$ & $29(1)$ & $16(1)$ & $12(1)$ & $18(1)$ \\
\hline$B(1)$ & $23(1)$ & $23(1)$ & $23(1)$ & $11(1)$ & $4(1)$ & $3(1)$ \\
\hline $\mathrm{N}(1)$ & $20(1)$ & $25(1)$ & $26(1)$ & $14(1)$ & $5(1)$ & $4(1)$ \\
\hline$N(2)$ & $18(1)$ & $22(1)$ & $24(1)$ & $11(1)$ & $5(1)$ & $3(1)$ \\
\hline
\end{tabular}




\begin{tabular}{lrrrrrr}
$\mathrm{N}(3)$ & $21(1)$ & $22(1)$ & $26(1)$ & $13(1)$ & $5(1)$ & $3(1)$ \\
$\mathrm{N}(4)$ & $19(1)$ & $20(1)$ & $24(1)$ & $10(1)$ & $5(1)$ & $4(1)$ \\
$\mathrm{N}(5)$ & $28(1)$ & $31(1)$ & $28(1)$ & $16(1)$ & $9(1)$ & $7(1)$ \\
$\mathrm{N}(6)$ & $28(1)$ & $46(1)$ & $43(1)$ & $27(1)$ & $12(1)$ & $4(1)$ \\
$\mathrm{P}(1)$ & $17(1)$ & $17(1)$ & $20(1)$ & $9(1)$ & $3(1)$ & $3(1)$ \\
$\mathrm{P}(2)$ & $19(1)$ & $19(1)$ & $21(1)$ & $11(1)$ & $5(1)$ & $4(1)$ \\
$\mathrm{Rh}(1)$ & $15(1)$ & $18(1)$ & $21(1)$ & $11(1)$ & $3(1)$ & $3(1)$ \\
$\mathrm{C}(46)$ & $36(3)$ & $85(4)$ & $52(5)$ & $25(4)$ & $18(3)$ & $-11(3)$ \\
$\mathrm{C}(47)$ & $48(3)$ & $64(4)$ & $51(3)$ & $3(3)$ & $23(2)$ & $1(3)$ \\
$\mathrm{C}(48)$ & $107(6)$ & $107(6)$ & $91(5)$ & $24(4)$ & $34(4)$ & $-51(5)$ \\
$\mathrm{C}(49)$ & $62(5)$ & $105(5)$ & $108(6)$ & $68(5)$ & $0(4)$ & $-27(4)$ \\
$\mathrm{C}(50)$ & $54(4)$ & $84(4)$ & $62(3)$ & $40(3)$ & $3(3)$ & $-14(3)$ \\
$\mathrm{C}(51)$ & $41(3)$ & $80(4)$ & $67(4)$ & $26(3)$ & $15(3)$ & $-17(3)$ \\
$\mathrm{C}(52)$ & $63(3)$ & $120(5)$ & $112(6)$ & $74(4)$ & $43(4)$ & $36(3)$ \\
$\mathrm{C}(46 \mathrm{~B})$ & $52(6)$ & $102(12)$ & $45(7)$ & $20(7)$ & $26(5)$ & $-1(6)$ \\
$\mathrm{C}(47 \mathrm{~B})$ & $115(12)$ & $85(10)$ & $125(12)$ & $69(9)$ & $45(9)$ & $-9(8)$ \\
$\mathrm{C}(48 \mathrm{~B})$ & $58(8)$ & $112(12)$ & $188(18)$ & $73(11)$ & $57(10)$ & $27(7)$ \\
$\mathrm{C}(49 \mathrm{~B})$ & $69(13)$ & $340(40)$ & $170(20)$ & $120(20)$ & $-10(13)$ & $-100(20)$ \\
$\mathrm{C}(50 \mathrm{~B})$ & $107(14)$ & $290(30)$ & $92(10)$ & $61(16)$ & $8(9)$ & $-109(18)$ \\
$\mathrm{C}(51 \mathrm{~B})$ & $62(7)$ & $120(10)$ & $57(7)$ & $-8(7)$ & $17(6)$ & $-52(7)$ \\
$\mathrm{C}(52 \mathrm{~B})$ & $52(5)$ & $300(20)$ & $127(12)$ & $149(14)$ & $26(6)$ & $10(8)$ \\
& & & & & & \\
\hline
\end{tabular}


Table S11. Hydrogen coordinates ( $\mathrm{x}$ 10^4) and isotropic displacement parameters $\left(A^{\wedge} 2 \times 10^{\wedge} 3\right)$ for complex 3 .

\begin{tabular}{|c|c|c|c|c|}
\hline & $x$ & Y & $\mathrm{z}$ & $\mathrm{U}(\mathrm{eq})$ \\
\hline $\mathrm{H}(2)$ & 635 & 1653 & 4207 & 27 \\
\hline $\mathrm{H}(3)$ & 913 & 512 & 5038 & 34 \\
\hline $\mathrm{H}(4)$ & 2201 & -784 & 4622 & 40 \\
\hline $\mathrm{H}(5)$ & 3249 & -895 & 3403 & 40 \\
\hline $\mathrm{H}(6)$ & 2956 & 230 & 2547 & 30 \\
\hline $\mathrm{H}(8)$ & 1131 & 4101 & 3917 & 28 \\
\hline $\mathrm{H}(9)$ & 2115 & 5447 & 5283 & 36 \\
\hline $\mathrm{H}(10)$ & 3959 & 5268 & 5820 & 43 \\
\hline $\mathrm{H}(11)$ & 4816 & 3728 & 5009 & 41 \\
\hline $\mathrm{H}(12)$ & 3823 & 2357 & 3679 & 32 \\
\hline $\mathrm{H}(14)$ & 3794 & 2220 & 2136 & 33 \\
\hline $\mathrm{H}(15)$ & 4607 & 1289 & 765 & 45 \\
\hline $\mathrm{H}(16)$ & 3610 & -88 & -557 & 45 \\
\hline H ( 17$)$ & 1759 & -500 & -533 & 39 \\
\hline H ( 18$)$ & 923 & 466 & 814 & 30 \\
\hline $\mathrm{H}(20)$ & 2322 & 4739 & 2764 & 31 \\
\hline $\mathrm{H}(21)$ & 4048 & 4927 & 2324 & 38 \\
\hline $\mathrm{H}(22)$ & 4352 & 4021 & 723 & 41 \\
\hline $\mathrm{H}(23)$ & 2954 & 2900 & -433 & 39 \\
\hline $\mathrm{H}(24)$ & 1224 & 2711 & -6 & 34 \\
\hline $\mathrm{H}(26)$ & -871 & 4664 & 3431 & 34 \\
\hline $\mathrm{H}(27)$ & -1245 & 6423 & 4293 & 49 \\
\hline $\mathrm{H}(28)$ & -631 & 7777 & 3857 & 50 \\
\hline $\mathrm{H}(29)$ & 408 & 7400 & 2591 & 41 \\
\hline $\mathrm{H}(30)$ & 805 & 5652 & 1736 & 32 \\
\hline $\mathrm{H}(32)$ & -1167 & 4851 & 833 & 33 \\
\hline $\mathrm{H}(33)$ & -2372 & 4418 & -633 & 42 \\
\hline H (34) & -2615 & 2675 & -1759 & 46 \\
\hline H (35) & -1645 & 1353 & -1425 & 43 \\
\hline $\mathrm{H}(36)$ & -485 & 1762 & 58 & 32 \\
\hline $\mathrm{H}(37)$ & -2558 & 3013 & 1049 & 35 \\
\hline $\mathrm{H}(38)$ & -4405 & 3158 & 1616 & 40 \\
\hline $\mathrm{H}(39)$ & -4131 & 2745 & 3132 & 33 \\
\hline $\mathrm{H}(40)$ & -198 & -358 & 1633 & 30 \\
\hline $\mathrm{H}(41)$ & -1344 & -1394 & 2282 & 37 \\
\hline $\mathrm{H}(42)$ & -2344 & -48 & 3497 & 35 \\
\hline H ( 43$)$ & -4828 & 1469 & 4948 & 50 \\
\hline $\mathrm{H}(44)$ & -3692 & 2879 & 6377 & 52 \\
\hline $\mathrm{H}(45)$ & -2017 & 3303 & 5761 & 40 \\
\hline H ( 47) & 5012 & 3015 & 9088 & 69 \\
\hline H ( 48$)$ & 4303 & 4386 & 8646 & 125 \\
\hline H ( 49$)$ & 3451 & 4021 & 7045 & 102 \\
\hline $\mathrm{H}(50)$ & 3309 & 2285 & 5887 & 76 \\
\hline $\mathrm{H}(51)$ & 4017 & 914 & 6329 & 75 \\
\hline $\mathrm{H}(52 \mathrm{~A})$ & 4851 & 285 & 7481 & 129 \\
\hline $\mathrm{H}(52 \mathrm{~B})$ & 4551 & 912 & 8548 & 129 \\
\hline $\mathrm{H}(52 \mathrm{C})$ & 5757 & 1089 & 8315 & 129 \\
\hline $\mathrm{H}(47 \mathrm{~B})$ & 4932 & 3664 & 9036 & 116 \\
\hline $\mathrm{H}(48 \mathrm{~B})$ & 3976 & 4225 & 7864 & 132 \\
\hline
\end{tabular}




\begin{tabular}{llrll} 
H (49B) & 3305 & 2987 & 6302 & 225 \\
$\mathrm{H}(50 \mathrm{~B})$ & 3590 & 1188 & 5913 & 201 \\
$\mathrm{H}(51 \mathrm{~B})$ & 4546 & 627 & 7084 & 109 \\
$\mathrm{H}(52 \mathrm{D})$ & 5396 & 948 & 8578 & 207 \\
$\mathrm{H}(52 \mathrm{E})$ & 4911 & 1882 & 9413 & 207 \\
$\mathrm{H}(52 \mathrm{~F})$ & 6091 & 2062 & 9136 & 207 \\
$\mathrm{H}(1)$ & $-1176(12)$ & $2756(12)$ & $4143(11)$ & $12(4)$ \\
\hline
\end{tabular}


Table S12. Torsion angles [deg] for complex 3.

\begin{tabular}{|c|c|}
\hline$C(6)-C(1)-C(2)-C(3)$ & $-2.3(2)$ \\
\hline $\mathrm{P}(1)-\mathrm{C}(1)-\mathrm{C}(2)-\mathrm{C}(3)$ & $-177.68(13)$ \\
\hline$C(1)-C(2)-C(3)-C(4)$ & $0.8(3)$ \\
\hline$C(2)-C(3)-C(4)-C(5)$ & $1.2(3)$ \\
\hline$C(3)-C(4)-C(5)-C(6)$ & $-1.7(3)$ \\
\hline$C(4)-C(5)-C(6)-C(1)$ & $0.2(3)$ \\
\hline$C(2)-C(1)-C(6)-C(5)$ & $1.7(2)$ \\
\hline$P(1)-C(1)-C(6)-C(5)$ & $176.93(14)$ \\
\hline$C(12)-C(7)-C(8)-C(9)$ & $1.7(2)$ \\
\hline$P(1)-C(7)-C(8)-C(9)$ & $172.33(13)$ \\
\hline$C(7)-C(8)-C(9)-C(10)$ & $0.1(3)$ \\
\hline$C(8)-C(9)-C(10)-C(11)$ & $-0.9(3)$ \\
\hline$C(9)-C(10)-C(11)-C(12)$ & $-0.3(3)$ \\
\hline$C(10)-C(11)-C(12)-C(7)$ & $2.2(3)$ \\
\hline$C(8)-C(7)-C(12)-C(11)$ & $-2.9(2)$ \\
\hline$P(1)-C(7)-C(12)-C(11)$ & $-173.07(14)$ \\
\hline$C(18)-C(13)-C(14)-C(15)$ & $-1.4(2)$ \\
\hline$P(1)-C(13)-C(14)-C(15)$ & $175.36(13)$ \\
\hline$C(13)-C(14)-C(15)-C(16)$ & $-0.5(3)$ \\
\hline$C(14)-C(15)-C(16)-C(17)$ & $1.3(3)$ \\
\hline$C(15)-C(16)-C(17)-C(18)$ & $-0.2(3)$ \\
\hline$C(16)-C(17)-C(18)-C(13)$ & $-1.8(3)$ \\
\hline$C(14)-C(13)-C(18)-C(17)$ & $2.6(2)$ \\
\hline$P(1)-C(13)-C(18)-C(17)$ & $-174.59(13)$ \\
\hline$C(24)-C(19)-C(20)-C(21)$ & $1.2(2)$ \\
\hline$P(2)-C(19)-C(20)-C(21)$ & $-179.26(12)$ \\
\hline$C(19)-C(20)-C(21)-C(22)$ & $-0.3(2)$ \\
\hline$C(20)-C(21)-C(22)-C(23)$ & $-0.8(3)$ \\
\hline$C(21)-C(22)-C(23)-C(24)$ & $0.9(3)$ \\
\hline$C(22)-C(23)-C(24)-C(19)$ & $0.0(3)$ \\
\hline$C(20)-C(19)-C(24)-C(23)$ & $-1.1(2)$ \\
\hline$P(2)-C(19)-C(24)-C(23)$ & $179.42(13)$ \\
\hline$C(30)-C(25)-C(26)-C(27)$ & $0.6(3)$ \\
\hline$P(2)-C(25)-C(26)-C(27)$ & $-175.88(14)$ \\
\hline$C(25)-C(26)-C(27)-C(28)$ & $0.4(3)$ \\
\hline$C(26)-C(27)-C(28)-C(29)$ & $-1.1(3)$ \\
\hline$C(27)-C(28)-C(29)-C(30)$ & $0.7(3)$ \\
\hline$C(28)-C(29)-C(30)-C(25)$ & $0.3(3)$ \\
\hline$C(26)-C(25)-C(30)-C(29)$ & $-1.0(2)$ \\
\hline$P(2)-C(25)-C(30)-C(29)$ & $175.42(14)$ \\
\hline$C(36)-C(31)-C(32)-C(33)$ & $0.1(3)$ \\
\hline$P(2)-C(31)-C(32)-C(33)$ & $-176.15(14)$ \\
\hline$C(31)-C(32)-C(33)-C(34)$ & $-0.6(3)$ \\
\hline$C(32)-C(33)-C(34)-C(35)$ & $0.0(3)$ \\
\hline$C(33)-C(34)-C(35)-C(36)$ & $1.2(3)$ \\
\hline$C(34)-C(35)-C(36)-C(31)$ & $-1.7(3)$ \\
\hline$C(32)-C(31)-C(36)-C(35)$ & $1.0(3)$ \\
\hline$P(2)-C(31)-C(36)-C(35)$ & $177.58(14)$ \\
\hline$N(1)-C(37)-C(38)-C(39)$ & $-0.2(2)$ \\
\hline $\mathrm{C}(37)-\mathrm{C}(38)-\mathrm{C}(39)-\mathrm{N}(2)$ & $0.2(2)$ \\
\hline$N(4)-C(40)-C(41)-C(42)$ & $0.23(19)$ \\
\hline$C(40)-C(41)-C(42)-N(3)$ & $-0.45(19)$ \\
\hline$N(6)-C(43)-C(44)-C(45)$ & $-0.1(2)$ \\
\hline$C(43)-C(44)-C(45)-N(5)$ & $0.2(2)$ \\
\hline
\end{tabular}




\begin{tabular}{|c|c|}
\hline $\mathrm{C}(38)-\mathrm{C}(37)-\mathrm{N}(1)-\mathrm{N}(2)$ & $0.2(2)$ \\
\hline $\mathrm{C}(38)-\mathrm{C}(37)-\mathrm{N}(1)-\mathrm{Rh}(1)$ & $177.75(13)$ \\
\hline $\mathrm{C}(38)-\mathrm{C}(39)-\mathrm{N}(2)-\mathrm{N}(1)$ & $-0.05(19)$ \\
\hline $\mathrm{C}(38)-\mathrm{C}(39)-\mathrm{N}(2)-\mathrm{B}(1)$ & $-175.80(16)$ \\
\hline $\mathrm{C}(37)-\mathrm{N}(1)-\mathrm{N}(2)-\mathrm{C}(39)$ & $-0.10(18)$ \\
\hline $\mathrm{Rh}(1)-\mathrm{N}(1)-\mathrm{N}(2)-\mathrm{C}(39)$ & $-178.21(11)$ \\
\hline $\mathrm{C}(37)-\mathrm{N}(1)-\mathrm{N}(2)-\mathrm{B}(1)$ & $176.10(14)$ \\
\hline $\mathrm{Rh}(1)-\mathrm{N}(1)-\mathrm{N}(2)-\mathrm{B}(1)$ & $-2.00(18)$ \\
\hline $\mathrm{N}(5)-\mathrm{B}(1)-\mathrm{N}(2)-\mathrm{C}(39)$ & $-5.5(2)$ \\
\hline $\mathrm{N}(3)-\mathrm{B}(1)-\mathrm{N}(2)-\mathrm{C}(39)$ & $115.21(18)$ \\
\hline $\mathrm{N}(5)-\mathrm{B}(1)-\mathrm{N}(2)-\mathrm{N}(1)$ & $179.14(13)$ \\
\hline $\mathrm{N}(3)-\mathrm{B}(1)-\mathrm{N}(2)-\mathrm{N}(1)$ & $-60.15(18)$ \\
\hline $\mathrm{C}(41)-\mathrm{C}(42)-\mathrm{N}(3)-\mathrm{N}(4)$ & $0.52(18)$ \\
\hline $\mathrm{C}(41)-\mathrm{C}(42)-\mathrm{N}(3)-\mathrm{B}(1)$ & $175.71(16)$ \\
\hline$N(5)-B(1)-N(3)-C(42)$ & $6.6(2)$ \\
\hline$N(2)-B(1)-N(3)-C(42)$ & $-113.13(18)$ \\
\hline $\mathrm{N}(5)-\mathrm{B}(1)-\mathrm{N}(3)-\mathrm{N}(4)$ & $-178.52(12)$ \\
\hline $\mathrm{N}(2)-\mathrm{B}(1)-\mathrm{N}(3)-\mathrm{N}(4)$ & $61.78(17)$ \\
\hline $\mathrm{C}(41)-\mathrm{C}(40)-\mathrm{N}(4)-\mathrm{N}(3)$ & $0.07(18)$ \\
\hline $\mathrm{C}(41)-\mathrm{C}(40)-\mathrm{N}(4)-\mathrm{Rh}(1)$ & $-174.50(11)$ \\
\hline$C(42)-N(3)-N(4)-C(40)$ & $-0.36(17)$ \\
\hline $\mathrm{B}(1)-\mathrm{N}(3)-\mathrm{N}(4)-\mathrm{C}(40)$ & $-176.37(13)$ \\
\hline $\mathrm{C}(42)-\mathrm{N}(3)-\mathrm{N}(4)-\mathrm{Rh}(1)$ & $174.97(10)$ \\
\hline $\mathrm{B}(1)-\mathrm{N}(3)-\mathrm{N}(4)-\mathrm{Rh}(1)$ & $-1.04(17)$ \\
\hline $\mathrm{C}(44)-\mathrm{C}(45)-\mathrm{N}(5)-\mathrm{N}(6)$ & $-0.20(19)$ \\
\hline $\mathrm{C}(44)-\mathrm{C}(45)-\mathrm{N}(5)-\mathrm{B}(1)$ & $176.43(15)$ \\
\hline $\mathrm{N}(3)-\mathrm{B}(1)-\mathrm{N}(5)-\mathrm{C}(45)$ & $128.26(16)$ \\
\hline$N(2)-B(1)-N(5)-C(45)$ & $-114.82(17)$ \\
\hline $\mathrm{N}(3)-\mathrm{B}(1)-\mathrm{N}(5)-\mathrm{N}(6)$ & $-55.50(19)$ \\
\hline $\mathrm{N}(2)-\mathrm{B}(1)-\mathrm{N}(5)-\mathrm{N}(6)$ & $61.42(19)$ \\
\hline$C(44)-C(43)-N(6)-N(5)$ & $0.0(2)$ \\
\hline$C(45)-N(5)-N(6)-C(43)$ & $0.14(18)$ \\
\hline $\mathrm{B}(1)-\mathrm{N}(5)-\mathrm{N}(6)-\mathrm{C}(43)$ & $-176.66(15)$ \\
\hline $\mathrm{C}(14)-\mathrm{C}(13)-\mathrm{P}(1)-\mathrm{C}(7)$ & $7.27(16)$ \\
\hline$C(18)-C(13)-P(1)-C(7)$ & $-175.87(11)$ \\
\hline $\mathrm{C}(14)-\mathrm{C}(13)-\mathrm{P}(1)-\mathrm{C}(1)$ & $-92.85(15)$ \\
\hline $\mathrm{C}(18)-\mathrm{C}(13)-\mathrm{P}(1)-\mathrm{C}(1)$ & $84.01(12)$ \\
\hline $\mathrm{C}(14)-\mathrm{C}(13)-\mathrm{P}(1)-\mathrm{Rh}(1)$ & $140.94(13)$ \\
\hline $\mathrm{C}(18)-\mathrm{C}(13)-\mathrm{P}(1)-\mathrm{Rh}(1)$ & $-42.20(13)$ \\
\hline$C(12)-C(7)-P(1)-C(13)$ & $-51.03(15)$ \\
\hline$C(8)-C(7)-P(1)-C(13)$ & $138.76(12)$ \\
\hline$C(12)-C(7)-P(1)-C(1)$ & $52.96(15)$ \\
\hline$C(8)-C(7)-P(1)-C(1)$ & $-117.25(13)$ \\
\hline $\mathrm{C}(12)-\mathrm{C}(7)-\mathrm{P}(1)-\mathrm{Rh}(1)$ & $175.33(12)$ \\
\hline $\mathrm{C}(8)-\mathrm{C}(7)-\mathrm{P}(1)-\mathrm{Rh}(1)$ & $5.12(14)$ \\
\hline$C(6)-C(1)-P(1)-C(13)$ & $13.62(15)$ \\
\hline$C(2)-C(1)-P(1)-C(13)$ & $-171.15(12)$ \\
\hline$C(6)-C(1)-P(1)-C(7)$ & $-97.13(14)$ \\
\hline$C(2)-C(1)-P(1)-C(7)$ & $78.10(13)$ \\
\hline $\mathrm{C}(6)-\mathrm{C}(1)-\mathrm{P}(1)-\mathrm{Rh}(1)$ & $140.12(12)$ \\
\hline $\mathrm{C}(2)-\mathrm{C}(1)-\mathrm{P}(1)-\mathrm{Rh}(1)$ & $-44.65(13)$ \\
\hline$C(26)-C(25)-P(2)-C(31)$ & $107.90(14)$ \\
\hline$C(30)-C(25)-P(2)-C(31)$ & $-68.45(14)$ \\
\hline$C(26)-C(25)-P(2)-C(19)$ & $-148.73(13)$ \\
\hline$C(30)-C(25)-P(2)-C(19)$ & $34.92(14)$ \\
\hline$C(26)-C(25)-P(2)-R h(1)$ & $-11 \cdot 39(15)$ \\
\hline$C(30)-C(25)-P(2)-R h(1)$ & $172.26(11)$ \\
\hline
\end{tabular}




\begin{tabular}{|c|c|}
\hline$C(36)-C(31)-P(2)-C(25)$ & $-170.89(13)$ \\
\hline$C(32)-C(31)-P(2)-C(25)$ & $5.42(16)$ \\
\hline$C(36)-C(31)-P(2)-C(19)$ & $85.36(14)$ \\
\hline$C(32)-C(31)-P(2)-C(19)$ & $-98.33(15)$ \\
\hline$C(36)-C(31)-P(2)-R h(1)$ & $-44.20(14)$ \\
\hline$C(32)-C(31)-P(2)-R h(1)$ & $132.10(13)$ \\
\hline$C(24)-C(19)-P(2)-C(25)$ & $-135.95(13)$ \\
\hline$C(20)-C(19)-P(2)-C(25)$ & $44.53(14)$ \\
\hline$C(24)-C(19)-P(2)-C(31)$ & $-31.24(14)$ \\
\hline$C(20)-C(19)-P(2)-C(31)$ & $149.24(13)$ \\
\hline$C(24)-C(19)-P(2)-R h(1)$ & $88.58(13)$ \\
\hline$C(20)-C(19)-P(2)-R h(1)$ & $-90.94(13)$ \\
\hline $\mathrm{C}(40)-\mathrm{N}(4)-\mathrm{Rh}(1)-\mathrm{N}(1)$ & $128.30(14)$ \\
\hline $\mathrm{N}(3)-\mathrm{N}(4)-\mathrm{Rh}(1)-\mathrm{N}(1)$ & $-45.59(11)$ \\
\hline $\mathrm{C}(40)-\mathrm{N}(4)-\mathrm{Rh}(1)-\mathrm{P}(1)$ & $-60.81(14)$ \\
\hline $\mathrm{N}(3)-\mathrm{N}(4)-\mathrm{Rh}(1)-\mathrm{P}(1)$ & $125.30(10)$ \\
\hline $\mathrm{C}(40)-\mathrm{N}(4)-\mathrm{Rh}(1)-\mathrm{P}(2)$ & $57.5(3)$ \\
\hline $\mathrm{N}(3)-\mathrm{N}(4)-\mathrm{Rh}(1)-\mathrm{P}(2)$ & $-116.38(18)$ \\
\hline $\mathrm{C}(37)-\mathrm{N}(1)-\mathrm{Rh}(1)-\mathrm{N}(4)$ & $-131.17(17)$ \\
\hline $\mathrm{N}(2)-\mathrm{N}(1)-\mathrm{Rh}(1)-\mathrm{N}(4)$ & $46.16(11)$ \\
\hline $\mathrm{C}(37)-\mathrm{N}(1)-\mathrm{Rh}(1)-\mathrm{P}(1)$ & $167.77(15)$ \\
\hline $\mathrm{N}(2)-\mathrm{N}(1)-\mathrm{Rh}(1)-\mathrm{P}(1)$ & $-14 \cdot 9(3)$ \\
\hline $\mathrm{C}(37)-\mathrm{N}(1)-\mathrm{Rh}(1)-\mathrm{P}(2)$ & $38.61(17)$ \\
\hline $\mathrm{N}(2)-\mathrm{N}(1)-\mathrm{Rh}(1)-\mathrm{P}(2)$ & $-144.06(10)$ \\
\hline $\mathrm{C}(13)-\mathrm{P}(1)-\mathrm{Rh}(1)-\mathrm{N}(4)$ & $104.30(7)$ \\
\hline $\mathrm{C}(7)-\mathrm{P}(1)-\mathrm{Rh}(1)-\mathrm{N}(4)$ & $-125.35(7)$ \\
\hline $\mathrm{C}(1)-\mathrm{P}(1)-\mathrm{Rh}(1)-\mathrm{N}(4)$ & $-14.25(7)$ \\
\hline $\mathrm{C}(13)-\mathrm{P}(1)-\mathrm{Rh}(1)-\mathrm{N}(1)$ & $164.7(2)$ \\
\hline $\mathrm{C}(7)-\mathrm{P}(1)-\mathrm{Rh}(1)-\mathrm{N}(1)$ & $-64.9(2)$ \\
\hline $\mathrm{C}(1)-\mathrm{P}(1)-\mathrm{Rh}(1)-\mathrm{N}(1)$ & $46.2(2)$ \\
\hline$C(13)-P(1)-R h(1)-P(2)$ & $-66.18(6)$ \\
\hline $\mathrm{C}(7)-\mathrm{P}(1)-\mathrm{Rh}(1)-\mathrm{P}(2)$ & $64.17(6)$ \\
\hline $\mathrm{C}(1)-\mathrm{P}(1)-\mathrm{Rh}(1)-\mathrm{P}(2)$ & $175.28(6)$ \\
\hline $\mathrm{C}(25)-\mathrm{P}(2)-\mathrm{Rh}(1)-\mathrm{N}(4)$ & $132.11(19)$ \\
\hline $\mathrm{C}(31)-\mathrm{P}(2)-\mathrm{Rh}(1)-\mathrm{N}(4)$ & $16.0(2)$ \\
\hline $\mathrm{C}(19)-\mathrm{P}(2)-\mathrm{Rh}(1)-\mathrm{N}(4)$ & $-100.0(2)$ \\
\hline $\mathrm{C}(25)-\mathrm{P}(2)-\mathrm{Rh}(1)-\mathrm{N}(1)$ & $62.17(7)$ \\
\hline $\mathrm{C}(31)-\mathrm{P}(2)-\mathrm{Rh}(1)-\mathrm{N}(1)$ & $-53.90(7)$ \\
\hline $\mathrm{C}(19)-\mathrm{P}(2)-\mathrm{Rh}(1)-\mathrm{N}(1)$ & $-169.91(7)$ \\
\hline$C(25)-P(2)-R h(1)-P(1)$ & $-109.75(6)$ \\
\hline $\mathrm{C}(31)-\mathrm{P}(2)-\mathrm{Rh}(1)-\mathrm{P}(1)$ & $134.17(5)$ \\
\hline $\mathrm{C}(19)-\mathrm{P}(2)-\mathrm{Rh}(1)-\mathrm{P}(1)$ & $18.17(6)$ \\
\hline$C(51)-C(46)-C(47)-C(48)$ & 0.0 \\
\hline$C(52)-C(46)-C(47)-C(48)$ & $-176.1(4)$ \\
\hline$C(46)-C(47)-C(48)-C(49)$ & 0.0 \\
\hline$C(47)-C(48)-C(49)-C(50)$ & 0.0 \\
\hline$C(48)-C(49)-C(50)-C(51)$ & 0.0 \\
\hline$C(49)-C(50)-C(51)-C(46)$ & 0.0 \\
\hline$C(47)-C(46)-C(51)-C(50)$ & 0.0 \\
\hline$C(52)-C(46)-C(51)-C(50)$ & $176.1(4)$ \\
\hline$C(51 B)-C(46 B)-C(47 B)-C(48 B)$ & 0.0 \\
\hline$C(52 B)-C(46 B)-C(47 B)-C(48 B)$ & $-176.2(9)$ \\
\hline$C(46 B)-C(47 B)-C(48 B)-C(49 B)$ & 0.0 \\
\hline$C(47 B)-C(48 B)-C(49 B)-C(50 B)$ & 0.0 \\
\hline$C(48 B)-C(49 B)-C(50 B)-C(51 B)$ & 0.0 \\
\hline$C(49 B)-C(50 B)-C(51 B)-C(46 B)$ & 0.0 \\
\hline$C(47 B)-C(46 B)-C(51 B)-C(50 B)$ & 0.0 \\
\hline
\end{tabular}


Figure S5. Crystal Structure of Complex 4 - Configuration represented in paper.

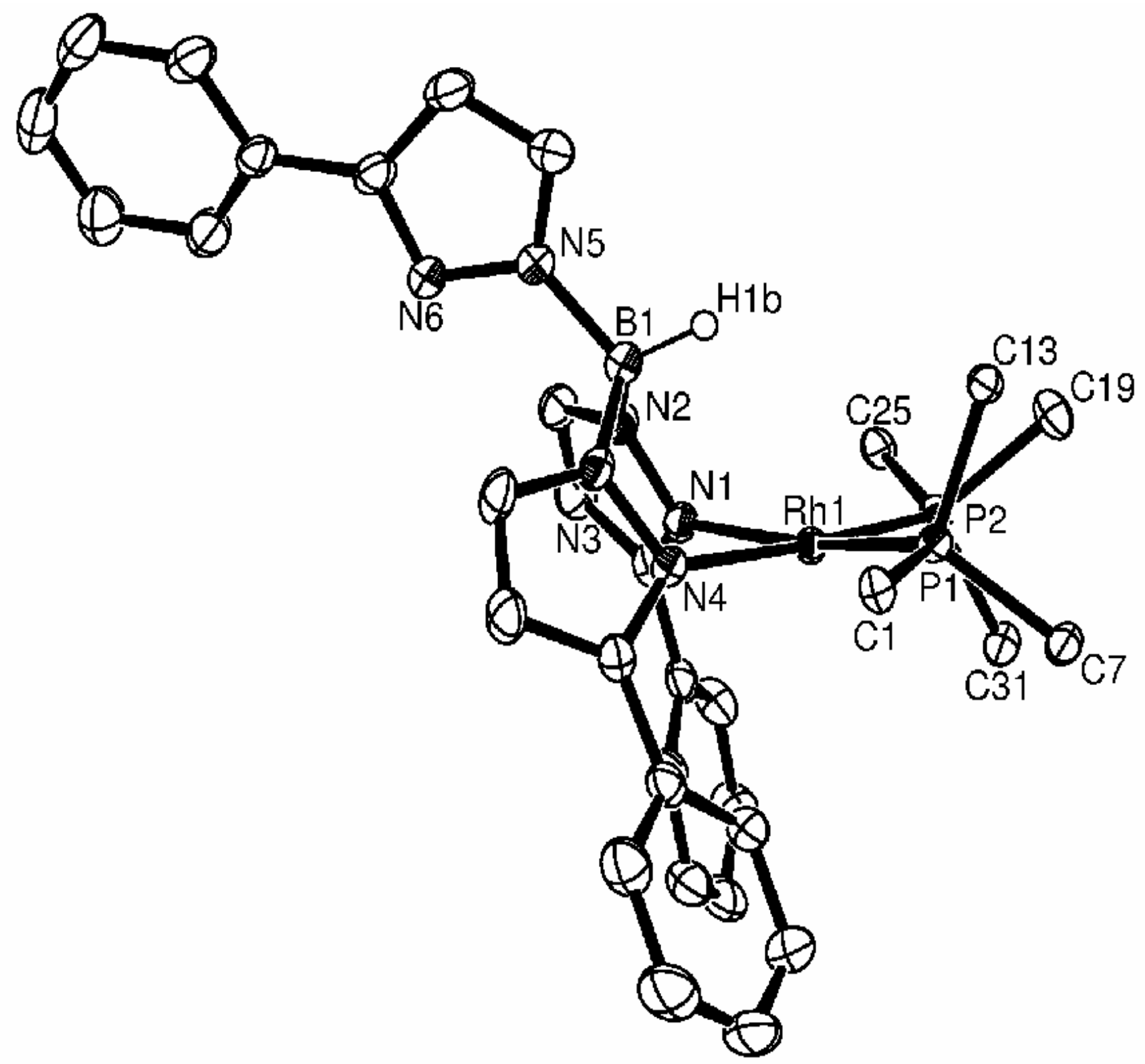


Figure S6. Crystal Structure of Complex 4 - Including all atoms.

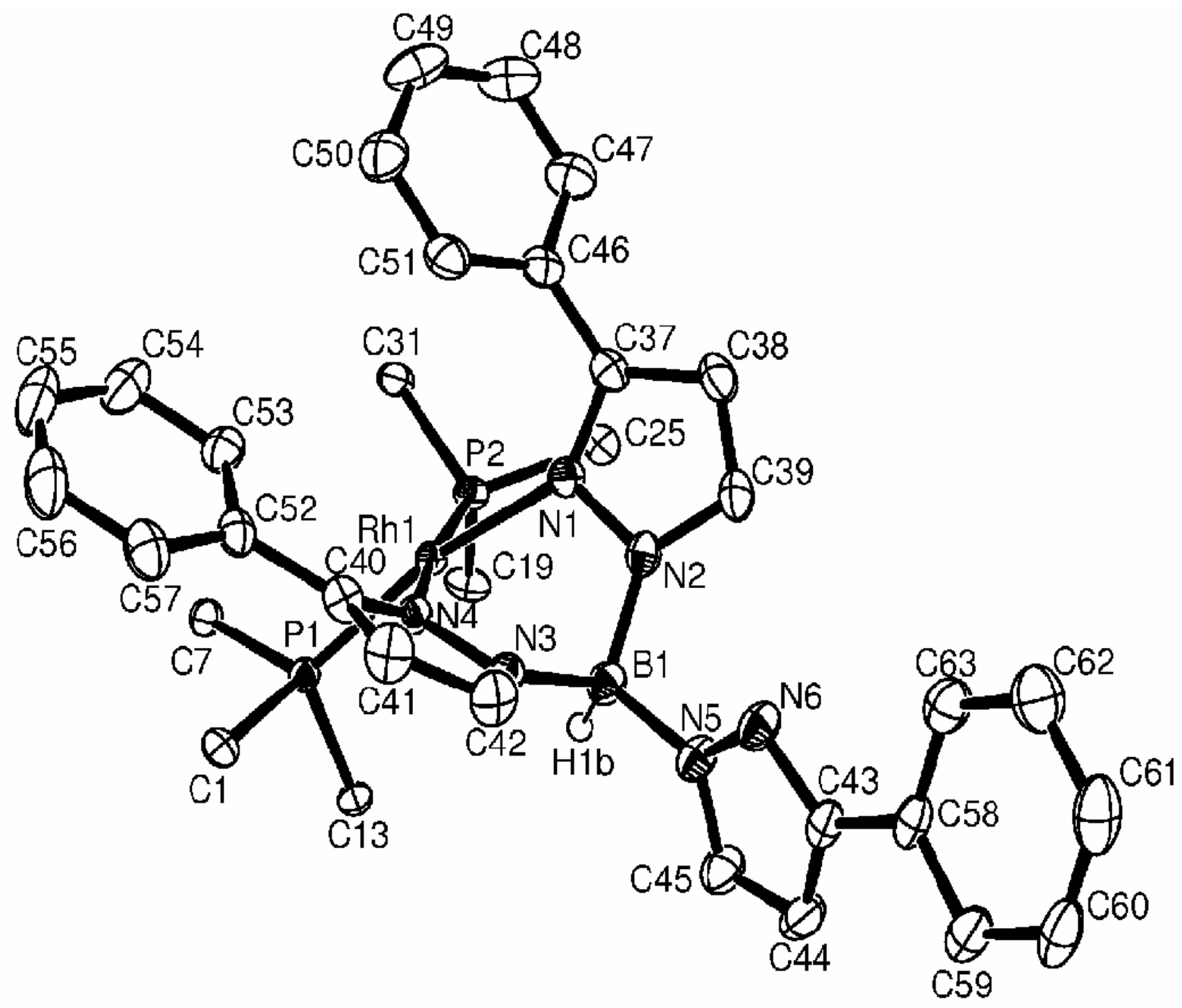




\section{Experimental}

\section{Data Collection}

An irregular orange crystal of $\mathrm{C}_{63} \mathrm{H}_{52} \mathrm{BN}_{6} \mathrm{P}_{2}$ Rh. $\mathrm{C}_{5} \mathrm{H}_{12}$ having approximate dimensions of $0.035 \times 0.10$ X $0.20 \mathrm{~mm}$ was mounted on a glass fiber. All measurements were made on a Bruker X8 APEX II diffractometer with graphite monochromated Mo-Ka radiation.

The data were collected at a temperature of $-100.0 \pm 0.1^{\circ} \mathrm{C}$ to a maximum $2 \theta$ value of $56.0^{\circ}$. Data were collected in a series of $\phi$ and $\omega$ scans in $0.50^{\circ}$ oscillations with 9.0 second exposures. The crystal-to-detector distance was $36.00 \mathrm{~mm}$.

\section{Data Reduction}

Of the 60965 reflections that were collected, 13758 were unique $\left(R_{i n t}=0.044\right)$; equivalent reflections were merged. Data were collected and integrated using the Bruker SAINT ${ }^{1}$ software package. The linear absorption coefficient, $\mu$, for Mo-K $\alpha$ radiation is $4.03 \mathrm{~cm}^{-1}$. Data were corrected for absorption effects using the multi-scan technique (SADABS ${ }^{2}$ ), with minimum and maximum transmission coefficients of 0.839 and 0.986 , respectively. The data were corrected for Lorentz and polarization effects.

\section{$\underline{\text { Structure Solution and Refinement }}$}

The structure was solved by direct methods ${ }^{3}$. The material crystallizes with one disordered molecule of solvent $\mathrm{C}_{5} \mathrm{H}_{12}$ in the asymmetric unit. This solvent molecule was modeled in two orientations with isotropic thermal parameters. All remaining non-hydrogen atoms were refined anisotropically, while all hydrogen atoms were placed in calculated positions and not refined, except for H1B which was located in a difference map and refined isotropically. The final cycle of full-matrix least-squares refinement ${ }^{4}$ on $\mathrm{F}^{2}$ was based on 13758 reflections and 701 variable parameters and converged (largest parameter shift was 0.00 times its esd) with unweighted and weighted agreement factors of:

$$
\begin{gathered}
\mathrm{R} 1=\Sigma\|\mathrm{Fo}|-| \mathrm{Fc}\| / \Sigma|\mathrm{Fo}|=0.053 \\
\mathrm{wR} 2=\left[\Sigma\left(\mathrm{w}\left(\mathrm{Fo}^{2}-\mathrm{Fc}^{2}\right)^{2}\right) / \Sigma \mathrm{w}\left(\mathrm{Fo}^{2}\right)^{2}\right]^{1 / 2}=0.093
\end{gathered}
$$


The standard deviation of an observation of unit weight ${ }^{5}$ was 1.02 . The weighting scheme was based on counting statistics. The maximum and minimum peaks on the final difference Fourier map corresponded to 0.82 and $-0.55 \mathrm{e}^{-} / \AA^{3}$, respectively.

Neutral atom scattering factors were taken from Cromer and Waber 6 . Anomalous dispersion effects were included in $\mathrm{Fcalc}^{7}$; the values for $\Delta \mathrm{f}^{\prime}$ and $\Delta \mathrm{f}^{\prime \prime}$ were those of Creagh and McAuley ${ }^{8}$. The values for the mass attenuation coefficients are those of Creagh and Hubbell ${ }^{9}$. All refinements were performed using the SHELXTL 10 crystallographic software package of Bruker-AXS.

\section{References}

(1) SAINT. Version 7.03A. Bruker AXS Inc., Madison, Wisconsin, USA. (1997-2003).

(2) SADABS. Bruker Nonius area detector scaling and absorption correction - V2.10, Bruker AXS Inc., Madison, Wisconsin, USA (2003).

(3) SIR97 - Altomare A., Burla M.C., Camalli M., Cascarano G.L., Giacovazzo C. , Guagliardi A., Moliterni A.G.G., Polidori G.,Spagna R. (1999) J. Appl. Cryst. 32, 115-119.

(4) Least Squares function minimized:

$$
\Sigma w\left(\mathrm{~F}_{\mathrm{O}}{ }^{2}-\mathrm{F}_{\mathrm{C}}{ }^{2}\right)^{2}
$$

(5) Standard deviation of an observation of unit weight:

$$
\begin{aligned}
& {\left[\Sigma w\left(\mathrm{~F}_{\mathrm{O}}^{2}-\mathrm{F}_{\mathrm{C}}^{2}\right)^{2} /\left(\mathrm{N}_{\mathrm{O}}-\mathrm{N}_{\mathrm{V}}\right)\right]^{1 / 2}} \\
& \text { where: } \mathrm{N}_{\mathrm{O}}=\text { number of observations } \\
& \qquad \mathrm{N}_{\mathrm{V}}=\text { number of variables }
\end{aligned}
$$

(6) Cromer, D. T. \& Waber, J. T.; "International Tables for X-ray Crystallography", Vol. IV, The Kynoch Press, Birmingham, England, Table 2.2 A (1974).

(7) Ibers, J. A. \& Hamilton, W. C.; Acta Crystallogr., 17, 781 (1964).

(8) Creagh, D. C. \& McAuley, W.J .; "International Tables for Crystallography", Vol C, (A.J.C. Wilson, ed.), Kluwer Academic Publishers, Boston, Table 4.2.6.8, pages 219-222 (1992).

(9) Creagh, D. C. \& Hubbell, J.H..; "International Tables for Crystallography", Vol C, (A.J.C. Wilson, ed.), Kluwer Academic Publishers, Boston, Table 4.2.4.3, pages 200-206 (1992).

(10) SHELXTL Version 5.1. Bruker AXS Inc., Madision, Wisconsin, USA. (1997). 
Table S13. Crystal Data and Structure Analysis Details for Complex 4.

A. Crystal Data

\section{Empirical Formula \\ Formula Weight \\ Crystal Color, Habit \\ Crystal Dimensions \\ Crystal System \\ Lattice Type \\ Lattice Parameters}

Space Group

$Z$ value

D calc

F000

$\mu(\operatorname{MoK} \alpha)$

\author{
$\mathrm{C}_{68} \mathrm{H}_{64} \mathrm{BN}_{6} \mathrm{P}_{2} \mathrm{Rh}$ \\ 1140.91 \\ orange, irregular \\ $0.035 \times 0.10 \times 0.20 \mathrm{~mm}$ \\ monoclinic \\ primitive \\ $a=17.4909(9) \AA$ \\ $\mathrm{b}=18.524(1) \AA$ \\ $\mathrm{c}=18.517(1) \AA$ \\ $\alpha=90.0^{\circ}$ \\ $\beta=107.914(5)^{\circ}$ \\ $\gamma=90.00$ \\ $\mathrm{V}=5708.7(5) \AA^{3}$
}

$P 21 / n$ (\#14)

4

$1.327 \mathrm{~g} / \mathrm{cm}^{3}$

2376.00

$4.03 \mathrm{~cm}^{-1}$ 
B. Intensity Measurements

Diffractometer

Radiation

Data Images

Detector Position

$2 \theta \max$

No. of Reflections Measured

Corrections
Bruker X8 APEX II

$\operatorname{MoK} \alpha(\lambda=0.71073 \AA)$

graphite monochromated

1220 exposures @ 9.0 seconds

$36.00 \mathrm{~mm}$

$56.0^{\circ}$

Total: 60965

Unique: $13758\left(R_{\text {int }}=0.044\right)$

Absorption $\left(T_{\min }=0.839, T_{\max }=0.986\right)$ Lorentz-polarization 
C. Structure Solution and Refinement

\begin{tabular}{|c|c|}
\hline Structure Solution & Direct Methods (SIR97) \\
\hline Refinement & Full-matrix least-squares on $\mathrm{F}^{2}$ \\
\hline Function Minimized & $\Sigma w\left(F o^{2}-F c^{2}\right)^{2}$ \\
\hline Least Squares Weights & $w=1 /\left(\sigma^{2}\left(F o^{2}\right)+(0.0396 P)^{2}+4.037 P\right)$ \\
\hline Anomalous Dispersion & All non-hydrogen atoms \\
\hline No. Observations $(I>0.00 \sigma(I))$ & 13758 \\
\hline No. Variables & 701 \\
\hline Reflection/Parameter Ratio & 19.63 \\
\hline Residuals (refined on $\mathrm{F}^{2}$, all data): R1; wR2 & $0.053 ; 0.093$ \\
\hline Goodness of Fit Indicator & 1.02 \\
\hline No. Observations $(I>2.00 \sigma(I))$ & 10811 \\
\hline Residuals (refined on F): R1; wR2 & $0.037 ; 0.085$ \\
\hline Max Shift/Error in Final Cycle & 0.00 \\
\hline Maximum peak in Final Diff. Map & $0.82 \mathrm{e}^{-} / \AA^{3}$ \\
\hline Minimum peak in Final Diff. Map & $-0.55 e^{-} / \AA^{3}$ \\
\hline
\end{tabular}


Table S14. Atomic coordinates $\left(\mathrm{x}^{\left.10^{\wedge} 4\right)}\right.$ ) and equivalent isotropic displacement parameters $\left(A^{\wedge} 2 \times 10^{\wedge} 3\right)$ for complex 4.

$\mathrm{U}(e q)$ is defined as one third of the trace of the orthogonalized Uij tensor.

\begin{tabular}{|c|c|c|c|c|}
\hline & $x$ & y & $\mathrm{z}$ & $\mathrm{U}(\mathrm{eq})$ \\
\hline$C(1)$ & $5586(1)$ & $1262(1)$ & 8085 (1) & $19(1)$ \\
\hline C (2) & $5659(1)$ & $1379(1)$ & 8845 (1) & $25(1)$ \\
\hline$C(3)$ & $6304(2)$ & $1098(2)$ & $9413(2)$ & $32(1)$ \\
\hline C (4) & $6869(2)$ & $680(2)$ & $9228(2)$ & $38(1)$ \\
\hline$C(5)$ & $6791(2)$ & $544(2)$ & $8480(2)$ & $36(1)$ \\
\hline$C(6)$ & $6157(1)$ & $839(1)$ & 7909 (1) & $27(1)$ \\
\hline$C(7)$ & $5152(1)$ & $1713(1)$ & $6555(1)$ & $21(1)$ \\
\hline$C(8)$ & $4933(2)$ & $1141(1)$ & $6052(1)$ & $27(1)$ \\
\hline$C(9)$ & $5202(2)$ & $1094(2)$ & $5425(2)$ & $39(1)$ \\
\hline$C(10)$ & $5696(2)$ & $1622(2)$ & $5293(2)$ & $46(1)$ \\
\hline$C(11)$ & $5916(2)$ & $2193(2)$ & $5785(2)$ & $45(1)$ \\
\hline $\mathrm{C}(12)$ & $5649(2)$ & $2243(2)$ & $6413(1)$ & $32(1)$ \\
\hline$C(13)$ & 4903 (1) & $2618(1)$ & $7774(1)$ & $18(1)$ \\
\hline$C(14)$ & $4244(1)$ & $2973(1)$ & 7866 (1) & $21(1)$ \\
\hline$C(15)$ & $4327(2)$ & $3634(1)$ & $8227(2)$ & $30(1)$ \\
\hline$C(16)$ & $5077(2)$ & $3947(1)$ & $8504(1)$ & $30(1)$ \\
\hline$C(17)$ & $5743(2)$ & $3598(1)$ & 8425 (1) & $26(1)$ \\
\hline$C(18)$ & $5662(1)$ & $2934(1)$ & $8070(1)$ & $22(1)$ \\
\hline C (19) & 3205 (1) & $2880(1)$ & 6092 (1) & $25(1)$ \\
\hline$C(20)$ & $2833(2)$ & 3462 (1) & $6314(2)$ & $33(1)$ \\
\hline$C(21)$ & $3144(2)$ & 4155 (2) & $6344(2)$ & $42(1)$ \\
\hline$C(22)$ & $3832(2)$ & $4267(2)$ & $6154(2)$ & $46(1)$ \\
\hline$C(23)$ & $4194(2)$ & $3704(2)$ & 5905 (2) & $43(1)$ \\
\hline$C(24)$ & $3881(2)$ & 3011 (2) & $5866(2)$ & $32(1)$ \\
\hline$C(25)$ & $1827(1)$ & 2087 (1) & $6053(1)$ & $22(1)$ \\
\hline$C(26)$ & $1232(1)$ & $2014(1)$ & $5360(1)$ & $28(1)$ \\
\hline C (27) & $439(2)$ & $2179(2)$ & $5293(2)$ & $39(1)$ \\
\hline C (28) & $237(2)$ & $2429(2)$ & $5910(2)$ & $44(1)$ \\
\hline C (29) & $825(2)$ & $2506(2)$ & $6604(2)$ & $38(1)$ \\
\hline$C(30)$ & 1609 (2) & $2330(1)$ & $6673(1)$ & $28(1)$ \\
\hline C (31) & $2903(1)$ & 1559 (1) & $5260(1)$ & $22(1)$ \\
\hline C (32) & $3008(2)$ & $817(1)$ & $5222(1)$ & $29(1)$ \\
\hline C (33) & $3058(2)$ & $492(2)$ & 4565 (2) & $36(1)$ \\
\hline C (34) & $3017(2)$ & $908(2)$ & $3939(2)$ & $40(1)$ \\
\hline$C(35)$ & 2901 (2) & $1640(2)$ & $3963(2)$ & $38(1)$ \\
\hline$C(36)$ & $2833(2)$ & $1967(2)$ & $4611(1)$ & $30(1)$ \\
\hline C (37) & $1751(1)$ & $558(1)$ & $6903(1)$ & $23(1)$ \\
\hline C (38) & $1152(1)$ & $624(1)$ & 7251 (2) & $30(1)$ \\
\hline C (39) & $1498(1)$ & $1000(1)$ & 7907 (2) & $27(1)$ \\
\hline$C(40)$ & $4334(1)$ & $15(1)$ & $8402(1)$ & $22(1)$ \\
\hline$C(41)$ & 4295 (2) & $-181(2)$ & $9120(1)$ & $31(1)$ \\
\hline C ( 42$)$ & $3838(2)$ & $335(2)$ & $9311(1)$ & $30(1)$ \\
\hline$C(43)$ & $2350(1)$ & $1412(1)$ & $10283(1)$ & $25(1)$ \\
\hline$C(44)$ & $2750(2)$ & $2077(2)$ & $10396(2)$ & $32(1)$ \\
\hline$C(45)$ & $2999(2)$ & $2164(1)$ & $9771(2)$ & $30(1)$ \\
\hline$C(46)$ & $1765(1)$ & $95(1)$ & $6265(1)$ & $24(1)$ \\
\hline C ( 47) & $1169(2)$ & $108(1)$ & $5567(2)$ & $32(1)$ \\
\hline
\end{tabular}




\begin{tabular}{|c|c|c|c|c|c|}
\hline$C(48)$ & $1188(2)$ & $-382(2)$ & $5009(2)$ & $42(1)$ & \\
\hline C (49) & $1792(2)$ & $-877(2)$ & $5133(2)$ & $43(1)$ & \\
\hline$C(50)$ & $2389(2)$ & $-901(2)$ & $5823(2)$ & $36(1)$ & \\
\hline C (51) & $2374(1)$ & $-417(1)$ & $6387(2)$ & $29(1)$ & \\
\hline C ( 52) & $4720(1)$ & $-386(1)$ & $7929(1)$ & $24(1)$ & \\
\hline C (53) & $4475(2)$ & $-315(1)$ & $7146(1)$ & $27(1)$ & \\
\hline$C(54)$ & $4830(2)$ & $-720(2)$ & $6709(2)$ & $39(1)$ & \\
\hline C (55) & $5427(2)$ & $-1206(2)$ & $7043(2)$ & $47(1)$ & \\
\hline$C(56)$ & $5674(2)$ & $-1282(2)$ & $7822(2)$ & $46(1)$ & \\
\hline C (57) & $5329(2)$ & $-877(2)$ & $8260(2)$ & $35(1)$ & \\
\hline C ( 58) & $1932(1)$ & $1051(1)$ & $10760(1)$ & $25(1)$ & \\
\hline C (59) & $2060(2)$ & $1262(2)$ & $11511(1)$ & $32(1)$ & \\
\hline$C(60)$ & $1649(2)$ & $937(2)$ & $11949(2)$ & $38(1)$ & \\
\hline$C(61)$ & $1113(2)$ & $391(2)$ & $11659(2)$ & $41(1)$ & \\
\hline$C(62)$ & $986(2)$ & $167(2)$ & $10917(2)$ & $39(1)$ & \\
\hline C (63) & $1392(2)$ & $500(2)$ & $10474(2)$ & $32(1)$ & \\
\hline$C(64)$ & $26(5)$ & $8158(4)$ & $992(4)$ & $103(3)$ & $0.667(6)$ \\
\hline$C(65)$ & $356(4)$ & $8394(5)$ & $1787(4)$ & $92(2)$ & $0.667(6)$ \\
\hline$C(66)$ & $1216(4)$ & $8339(5)$ & $2078(4)$ & $104(3)$ & $0.667(6)$ \\
\hline C (67) & $1361(4)$ & $8606(5)$ & $2923(4)$ & $106(3)$ & $0.667(6)$ \\
\hline$C(68)$ & $2193(4)$ & $8810(4)$ & $3132(4)$ & $155(3)$ & \\
\hline $\mathrm{B}(1)$ & $2988(2)$ & $1441(2)$ & $8602(2)$ & $24(1)$ & \\
\hline $\mathrm{N}(2)$ & $2259(1)$ & $1157(1)$ & $7948(1)$ & $23(1)$ & \\
\hline$N(1)$ & $2413(1)$ & $898(1)$ & $7318(1)$ & $20(1)$ & \\
\hline$N(5)$ & $2761(1)$ & $1588(1)$ & $9316(1)$ & $25(1)$ & \\
\hline$N(6)$ & $2357(1)$ & $1115(1)$ & $9629(1)$ & $26(1)$ & \\
\hline$N(3)$ & $3621(1)$ & $826(1)$ & $8750(1)$ & $21(1)$ & \\
\hline$N(4)$ & $3927(1)$ & $632(1)$ & $8185(1)$ & $19(1)$ & \\
\hline$P(1)$ & $4757(1)$ & $1708(1)$ & $7365(1)$ & $17(1)$ & \\
\hline$P(2)$ & $2894(1)$ & $1941(1)$ & $6170(1)$ & $19(1)$ & \\
\hline $\operatorname{Rh}(1)$ & $3519(1)$ & $1272(1)$ & $7206(1)$ & $16(1)$ & \\
\hline$C(67 B)$ & $2119(6)$ & $8294(5)$ & $2456(6)$ & $64(3)$ & $0.333(6)$ \\
\hline$C(66 \mathrm{~B})$ & $1534(7)$ & $7756(5)$ & $2619(9)$ & $111(6)$ & $0.333(6)$ \\
\hline$C(65 B)$ & $770(7)$ & $8266(9)$ & $2361(9)$ & $98(5)$ & $0.333(6)$ \\
\hline$C(64 B)$ & $850(20)$ & 8377 (19) & $1537(11)$ & $266(18)$ & $0.333(6)$ \\
\hline
\end{tabular}


Table S15. Bond lengths [A] and angles [deg] for complex 4.

\begin{tabular}{|c|c|}
\hline$C(1)-C(6)$ & $1.385(3)$ \\
\hline$C(1)-C(2)$ & $1.390(3)$ \\
\hline$C(1)-P(1)$ & $1.838(2)$ \\
\hline$C(2)-C(3)$ & $1.386(3)$ \\
\hline $\mathrm{C}(2)-\mathrm{H}(2)$ & 0.9500 \\
\hline$C(3)-C(4)$ & $1.379(4)$ \\
\hline $\mathrm{C}(3)-\mathrm{H}(3)$ & 0.9500 \\
\hline$C(4)-C(5)$ & $1.373(4)$ \\
\hline $\mathrm{C}(4)-\mathrm{H}(4)$ & 0.9500 \\
\hline$C(5)-C(6)$ & $1.388(3)$ \\
\hline $\mathrm{C}(5)-\mathrm{H}(5)$ & 0.9500 \\
\hline $\mathrm{C}(6)-\mathrm{H}(6)$ & 0.9500 \\
\hline$C(7)-C(8)$ & $1.385(3)$ \\
\hline$C(7)-C(12)$ & $1.390(3)$ \\
\hline$C(7)-P(1)$ & $1.833(2)$ \\
\hline$C(8)-C(9)$ & $1.383(3)$ \\
\hline $\mathrm{C}(8)-\mathrm{H}(8)$ & 0.9500 \\
\hline$C(9)-C(10)$ & $1.375(4)$ \\
\hline $\mathrm{C}(9)-\mathrm{H}(9)$ & 0.9500 \\
\hline$C(10)-C(11)$ & $1.372(4)$ \\
\hline $\mathrm{C}(10)-\mathrm{H}(10)$ & 0.9500 \\
\hline$C(11)-C(12)$ & $1.383(4)$ \\
\hline $\mathrm{C}(11)-\mathrm{H}(11)$ & 0.9500 \\
\hline $\mathrm{C}(12)-\mathrm{H}(12)$ & 0.9500 \\
\hline$C(13)-C(14)$ & $1.383(3)$ \\
\hline$C(13)-C(18)$ & $1.400(3)$ \\
\hline$C(13)-P(1)$ & $1.833(2)$ \\
\hline$C(14)-C(15)$ & $1.382(3)$ \\
\hline $\mathrm{C}(14)-\mathrm{H}(14)$ & 0.9500 \\
\hline$C(15)-C(16)$ & $1.380(4)$ \\
\hline $\mathrm{C}(15)-\mathrm{H}(15)$ & 0.9500 \\
\hline$C(16)-C(17)$ & $1.379(4)$ \\
\hline $\mathrm{C}(16)-\mathrm{H}(16)$ & 0.9500 \\
\hline $\mathrm{C}(17)-\mathrm{C}(18)$ & $1.381(3)$ \\
\hline $\mathrm{C}(17)-\mathrm{H}(17)$ & 0.9500 \\
\hline $\mathrm{C}(18)-\mathrm{H}(18)$ & 0.9500 \\
\hline$C(19)-C(20)$ & $1.385(4)$ \\
\hline$C(19)-C(24)$ & $1.390(4)$ \\
\hline$C(19)-P(2)$ & $1.843(2)$ \\
\hline$C(20)-C(21)$ & $1.387(4)$ \\
\hline $\mathrm{C}(20)-\mathrm{H}(20)$ & 0.9500 \\
\hline$C(21)-C(22)$ & $1.370(4)$ \\
\hline $\mathrm{C}(21)-\mathrm{H}(21)$ & 0.9500 \\
\hline$C(22)-C(23)$ & $1.370(5)$ \\
\hline $\mathrm{C}(22)-\mathrm{H}(22)$ & 0.9500 \\
\hline$C(23)-C(24)$ & $1.390(4)$ \\
\hline $\mathrm{C}(23)-\mathrm{H}(23)$ & 0.9500 \\
\hline $\mathrm{C}(24)-\mathrm{H}(24)$ & 0.9500 \\
\hline$C(25)-C(26)$ & $1.387(3)$ \\
\hline$C(25)-C(30)$ & $1.391(3)$ \\
\hline$C(25)-P(2)$ & $1.831(2)$ \\
\hline$C(26)-C(27)$ & $1.388(4)$ \\
\hline $\mathrm{C}(26)-\mathrm{H}(26)$ & 0.9500 \\
\hline
\end{tabular}




\begin{tabular}{|c|c|}
\hline$C(27)-C(28)$ & $1.375(4)$ \\
\hline $\mathrm{C}(27)-\mathrm{H}(27)$ & 0.9500 \\
\hline$C(28)-C(29)$ & $1.385(4)$ \\
\hline $\mathrm{C}(28)-\mathrm{H}(28)$ & 0.9500 \\
\hline$C(29)-C(30)$ & $1.377(4)$ \\
\hline $\mathrm{C}(29)-\mathrm{H}(29)$ & 0.9500 \\
\hline $\mathrm{C}(30)-\mathrm{H}(30)$ & 0.9500 \\
\hline$C(31)-C(32)$ & $1.390(4)$ \\
\hline$C(31)-C(36)$ & $1.394(3)$ \\
\hline $\mathrm{C}(31)-\mathrm{P}(2)$ & $1.832(2)$ \\
\hline$C(32)-C(33)$ & $1.385(4)$ \\
\hline $\mathrm{C}(32)-\mathrm{H}(32)$ & 0.9500 \\
\hline$C(33)-C(34)$ & $1.376(4)$ \\
\hline $\mathrm{C}(33)-\mathrm{H}(33)$ & 0.9500 \\
\hline$C(34)-C(35)$ & $1.375(4)$ \\
\hline $\mathrm{C}(34)-\mathrm{H}(34)$ & 0.9500 \\
\hline$C(35)-C(36)$ & $1.381(4)$ \\
\hline $\mathrm{C}(35)-\mathrm{H}(35)$ & 0.9500 \\
\hline $\mathrm{C}(36)-\mathrm{H}(36)$ & 0.9500 \\
\hline $\mathrm{C}(37)-\mathrm{N}(1)$ & $1.335(3)$ \\
\hline$C(37)-C(38)$ & $1.394(3)$ \\
\hline$C(37)-C(46)$ & $1.466(3)$ \\
\hline$C(38)-C(39)$ & $1.370(4)$ \\
\hline $\mathrm{C}(38)-\mathrm{H}(38)$ & 0.9500 \\
\hline $\mathrm{C}(39)-\mathrm{N}(2)$ & $1.343(3)$ \\
\hline $\mathrm{C}(39)-\mathrm{H}(39)$ & 0.9500 \\
\hline $\mathrm{C}(40)-\mathrm{N}(4)$ & $1.341(3)$ \\
\hline$C(40)-C(41)$ & $1.400(3)$ \\
\hline$C(40)-C(52)$ & $1.462(3)$ \\
\hline$C(41)-C(42)$ & $1.362(4)$ \\
\hline $\mathrm{C}(41)-\mathrm{H}(41)$ & 0.9500 \\
\hline $\mathrm{C}(42)-\mathrm{N}(3)$ & $1.345(3)$ \\
\hline $\mathrm{C}(42)-\mathrm{H}(42)$ & 0.9500 \\
\hline $\mathrm{C}(43)-\mathrm{N}(6)$ & $1.332(3)$ \\
\hline$C(43)-C(44)$ & $1.400(4)$ \\
\hline$C(43)-C(58)$ & $1.470(3)$ \\
\hline$C(44)-C(45)$ & $1.366(4)$ \\
\hline $\mathrm{C}(44)-\mathrm{H}(44)$ & 0.9500 \\
\hline $\mathrm{C}(45)-\mathrm{N}(5)$ & $1.344(3)$ \\
\hline $\mathrm{C}(45)-\mathrm{H}(45)$ & 0.9500 \\
\hline$C(46)-C(47)$ & $1.388(3)$ \\
\hline$C(46)-C(51)$ & $1.392(3)$ \\
\hline$C(47)-C(48)$ & $1.383(4)$ \\
\hline $\mathrm{C}(47)-\mathrm{H}(47)$ & 0.9500 \\
\hline$C(48)-C(49)$ & $1.365(4)$ \\
\hline $\mathrm{C}(48)-\mathrm{H}(48)$ & 0.9500 \\
\hline$C(49)-C(50)$ & $1.379(4)$ \\
\hline $\mathrm{C}(49)-\mathrm{H}(49)$ & 0.9500 \\
\hline$C(50)-C(51)$ & $1.382(4)$ \\
\hline $\mathrm{C}(50)-\mathrm{H}(50)$ & 0.9500 \\
\hline $\mathrm{C}(51)-\mathrm{H}(51)$ & 0.9500 \\
\hline$C(52)-C(53)$ & $1.387(3)$ \\
\hline$C(52)-C(57)$ & $1.390(3)$ \\
\hline$C(53)-C(54)$ & $1.382(3)$ \\
\hline $\mathrm{C}(53)-\mathrm{H}(53)$ & 0.9500 \\
\hline$C(54)-C(55)$ & $1.374(4)$ \\
\hline $\mathrm{C}(54)-\mathrm{H}(54)$ & 0.9500 \\
\hline
\end{tabular}




\begin{tabular}{|c|c|}
\hline$C(55)-C(56)$ & $1.379(5)$ \\
\hline $\mathrm{C}(55)-\mathrm{H}(55)$ & 0.9500 \\
\hline$C(56)-C(57)$ & $1.374(4)$ \\
\hline $\mathrm{C}(56)-\mathrm{H}(56)$ & 0.9500 \\
\hline $\mathrm{C}(57)-\mathrm{H}(57)$ & 0.9500 \\
\hline$C(58)-C(63)$ & $1.381(4)$ \\
\hline$C(58)-C(59)$ & $1.395(3)$ \\
\hline$C(59)-C(60)$ & $1.376(4)$ \\
\hline $\mathrm{C}(59)-\mathrm{H}(59)$ & 0.9500 \\
\hline$C(60)-C(61)$ & $1.372(4)$ \\
\hline $\mathrm{C}(60)-\mathrm{H}(60)$ & 0.9500 \\
\hline$C(61)-C(62)$ & $1.385(4)$ \\
\hline $\mathrm{C}(61)-\mathrm{H}(61)$ & 0.9500 \\
\hline$C(62)-C(63)$ & $1.385(4)$ \\
\hline $\mathrm{C}(62)-\mathrm{H}(62)$ & 0.9500 \\
\hline $\mathrm{C}(63)-\mathrm{H}(63)$ & 0.9500 \\
\hline$C(64)-C(65)$ & $1.473(7)$ \\
\hline $\mathrm{C}(64)-\mathrm{H}(64 \mathrm{~A})$ & 0.9800 \\
\hline $\mathrm{C}(64)-\mathrm{H}(64 \mathrm{~B})$ & 0.9800 \\
\hline $\mathrm{C}(64)-\mathrm{H}(64 \mathrm{C})$ & 0.9800 \\
\hline$C(65)-C(66)$ & $1.438(7)$ \\
\hline $\mathrm{C}(65)-\mathrm{H}(65 \mathrm{~A})$ & 0.9900 \\
\hline$C(65)-H(65 B)$ & 0.9900 \\
\hline$C(66)-C(67)$ & $1.586(7)$ \\
\hline $\mathrm{C}(66)-\mathrm{H}(66 \mathrm{~A})$ & 0.9900 \\
\hline $\mathrm{C}(66)-\mathrm{H}(66 \mathrm{~B})$ & 0.9900 \\
\hline $\mathrm{C}(66)-\mathrm{H}(67 \mathrm{C})$ & $1.334(8)$ \\
\hline$C(67)-C(68)$ & $1.436(7)$ \\
\hline$C(67)-H(67 A)$ & 0.9900 \\
\hline$C(67)-H(67 B)$ & 0.9900 \\
\hline $\mathrm{C}(68)-\mathrm{H}(68 \mathrm{~A})$ & 0.9800 \\
\hline $\mathrm{C}(68)-\mathrm{H}(68 \mathrm{~B})$ & 0.9800 \\
\hline $\mathrm{C}(68)-\mathrm{H}(68 \mathrm{C})$ & 0.9800 \\
\hline $\mathrm{B}(1)-\mathrm{N}(5)$ & $1.518(3)$ \\
\hline $\mathrm{B}(1)-\mathrm{N}(3)$ & $1.553(3)$ \\
\hline $\mathrm{B}(1)-\mathrm{N}(2)$ & $1.555(3)$ \\
\hline $\mathrm{B}(1)-\mathrm{H}(1 \mathrm{~B})$ & $1.08(2)$ \\
\hline$N(2)-N(1)$ & $1.363(3)$ \\
\hline $\mathrm{N}(1)-\mathrm{Rh}(1)$ & $2.1240(18)$ \\
\hline$N(5)-N(6)$ & $1.362(3)$ \\
\hline$N(3)-N(4)$ & $1.361(2)$ \\
\hline $\mathrm{N}(4)-\mathrm{Rh}(1)$ & $2.1000(18)$ \\
\hline $\mathrm{P}(1)-\mathrm{Rh}(1)$ & $2.2429(6)$ \\
\hline$P(2)-R h(1)$ & $2.2618(6)$ \\
\hline$C(67 B)-C(66 B)$ & $1.523(10)$ \\
\hline$C(67 B)-H(67 C)$ & $0.947(12)$ \\
\hline$C(67 B)-H(67 D)$ & $1.013(10)$ \\
\hline$C(66 B)-C(65 B)$ & $1.585(10)$ \\
\hline$C(66 B)-H(66 C)$ & 0.9900 \\
\hline$C(66 B)-H(66 D)$ & 0.9900 \\
\hline$C(65 B)-C(64 B)$ & $1.585(11)$ \\
\hline$C(65 B)-H(65 C)$ & 0.9900 \\
\hline$C(65 B)-H(65 D)$ & 0.9900 \\
\hline$C(64 B)-H(64 D)$ & 0.9800 \\
\hline $\mathrm{C}(64 \mathrm{~B})-\mathrm{H}(64 \mathrm{E})$ & 0.9800 \\
\hline$C(64 B)-H(64 F)$ & 0.9800 \\
\hline
\end{tabular}




\begin{tabular}{|c|c|}
\hline$C(6)-C(1)-C(2)$ & $118.6(2)$ \\
\hline$C(6)-C(1)-P(1)$ & $123.29(18)$ \\
\hline$C(2)-C(1)-P(1)$ & $118.09(17)$ \\
\hline$C(3)-C(2)-C(1)$ & $120.7(2)$ \\
\hline $\mathrm{C}(3)-\mathrm{C}(2)-\mathrm{H}(2)$ & 119.7 \\
\hline $\mathrm{C}(1)-\mathrm{C}(2)-\mathrm{H}(2)$ & 119.7 \\
\hline$C(4)-C(3)-C(2)$ & $120.0(2)$ \\
\hline $\mathrm{C}(4)-\mathrm{C}(3)-\mathrm{H}(3)$ & 120.0 \\
\hline$C(2)-C(3)-H(3)$ & 120.0 \\
\hline$C(5)-C(4)-C(3)$ & $119.8(2)$ \\
\hline $\mathrm{C}(5)-\mathrm{C}(4)-\mathrm{H}(4)$ & 120.1 \\
\hline $\mathrm{C}(3)-\mathrm{C}(4)-\mathrm{H}(4)$ & 120.1 \\
\hline$C(4)-C(5)-C(6)$ & $120.3(2)$ \\
\hline $\mathrm{C}(4)-\mathrm{C}(5)-\mathrm{H}(5)$ & 119.9 \\
\hline$C(6)-C(5)-H(5)$ & 119.9 \\
\hline$C(1)-C(6)-C(5)$ & $120.6(2)$ \\
\hline $\mathrm{C}(1)-\mathrm{C}(6)-\mathrm{H}(6)$ & 119.7 \\
\hline$C(5)-C(6)-H(6)$ & 119.7 \\
\hline$C(8)-C(7)-C(12)$ & $118.2(2)$ \\
\hline$C(8)-C(7)-P(1)$ & $116.85(17)$ \\
\hline$C(12)-C(7)-P(1)$ & $124.97(18)$ \\
\hline$C(9)-C(8)-C(7)$ & $121.2(2)$ \\
\hline $\mathrm{C}(9)-\mathrm{C}(8)-\mathrm{H}(8)$ & 119.4 \\
\hline $\mathrm{C}(7)-\mathrm{C}(8)-\mathrm{H}(8)$ & 119.4 \\
\hline$C(10)-C(9)-C(8)$ & $119.9(2)$ \\
\hline $\mathrm{C}(10)-\mathrm{C}(9)-\mathrm{H}(9)$ & 120.0 \\
\hline $\mathrm{C}(8)-\mathrm{C}(9)-\mathrm{H}(9)$ & 120.0 \\
\hline$C(11)-C(10)-C(9)$ & $119.6(2)$ \\
\hline $\mathrm{C}(11)-\mathrm{C}(10)-\mathrm{H}(10)$ & 120.2 \\
\hline$C(9)-C(10)-H(10)$ & 120.2 \\
\hline$C(10)-C(11)-C(12)$ & $120.8(3)$ \\
\hline $\mathrm{C}(10)-\mathrm{C}(11)-\mathrm{H}(11)$ & 119.6 \\
\hline $\mathrm{C}(12)-\mathrm{C}(11)-\mathrm{H}(11)$ & 119.6 \\
\hline $\mathrm{C}(11)-\mathrm{C}(12)-\mathrm{C}(7)$ & $120.3(2)$ \\
\hline $\mathrm{C}(11)-\mathrm{C}(12)-\mathrm{H}(12)$ & 119.9 \\
\hline $\mathrm{C}(7)-\mathrm{C}(12)-\mathrm{H}(12)$ & 119.9 \\
\hline$C(14)-C(13)-C(18)$ & $118.6(2)$ \\
\hline $\mathrm{C}(14)-\mathrm{C}(13)-\mathrm{P}(1)$ & $118.17(17)$ \\
\hline $\mathrm{C}(18)-\mathrm{C}(13)-\mathrm{P}(1)$ & $122.83(17)$ \\
\hline$C(15)-C(14)-C(13)$ & $120.8(2)$ \\
\hline $\mathrm{C}(15)-\mathrm{C}(14)-\mathrm{H}(14)$ & 119.6 \\
\hline $\mathrm{C}(13)-\mathrm{C}(14)-\mathrm{H}(14)$ & 119.6 \\
\hline$C(16)-C(15)-C(14)$ & $120.0(2)$ \\
\hline $\mathrm{C}(16)-\mathrm{C}(15)-\mathrm{H}(15)$ & 120.0 \\
\hline $\mathrm{C}(14)-\mathrm{C}(15)-\mathrm{H}(15)$ & 120.0 \\
\hline$C(17)-C(16)-C(15)$ & $120.0(2)$ \\
\hline $\mathrm{C}(17)-\mathrm{C}(16)-\mathrm{H}(16)$ & 120.0 \\
\hline $\mathrm{C}(15)-\mathrm{C}(16)-\mathrm{H}(16)$ & 120.0 \\
\hline$C(16)-C(17)-C(18)$ & $120.2(2)$ \\
\hline $\mathrm{C}(16)-\mathrm{C}(17)-\mathrm{H}(17)$ & 119.9 \\
\hline $\mathrm{C}(18)-\mathrm{C}(17)-\mathrm{H}(17)$ & 119.9 \\
\hline$C(17)-C(18)-C(13)$ & $120.3(2)$ \\
\hline $\mathrm{C}(17)-\mathrm{C}(18)-\mathrm{H}(18)$ & 119.8 \\
\hline $\mathrm{C}(13)-\mathrm{C}(18)-\mathrm{H}(18)$ & 119.8 \\
\hline$C(20)-C(19)-C(24)$ & $118.3(2)$ \\
\hline$C(20)-C(19)-P(2)$ & $122.3(2)$ \\
\hline $\mathrm{C}(24)-\mathrm{C}(19)-\mathrm{P}(2)$ & $119.1(2)$ \\
\hline
\end{tabular}




\begin{tabular}{|c|c|}
\hline$C(19)-C(20)-C(21)$ & $121.2(3)$ \\
\hline $\mathrm{C}(19)-\mathrm{C}(20)-\mathrm{H}(20)$ & 119.4 \\
\hline $\mathrm{C}(21)-\mathrm{C}(20)-\mathrm{H}(20)$ & 119.4 \\
\hline$C(22)-C(21)-C(20)$ & $119.6(3)$ \\
\hline $\mathrm{C}(22)-\mathrm{C}(21)-\mathrm{H}(21)$ & 120.2 \\
\hline $\mathrm{C}(20)-\mathrm{C}(21)-\mathrm{H}(21)$ & 120.2 \\
\hline$C(21)-C(22)-C(23)$ & $120.3(3)$ \\
\hline $\mathrm{C}(21)-\mathrm{C}(22)-\mathrm{H}(22)$ & 119.8 \\
\hline $\mathrm{C}(23)-\mathrm{C}(22)-\mathrm{H}(22)$ & 119.8 \\
\hline$C(22)-C(23)-C(24)$ & $120.3(3)$ \\
\hline $\mathrm{C}(22)-\mathrm{C}(23)-\mathrm{H}(23)$ & 119.8 \\
\hline $\mathrm{C}(24)-\mathrm{C}(23)-\mathrm{H}(23)$ & 119.8 \\
\hline$C(19)-C(24)-C(23)$ & $120.2(3)$ \\
\hline $\mathrm{C}(19)-\mathrm{C}(24)-\mathrm{H}(24)$ & 119.9 \\
\hline $\mathrm{C}(23)-\mathrm{C}(24)-\mathrm{H}(24)$ & 119.9 \\
\hline$C(26)-C(25)-C(30)$ & $118.5(2)$ \\
\hline$C(26)-C(25)-P(2)$ & $122.86(19)$ \\
\hline$C(30)-C(25)-P(2)$ & $118.53(18)$ \\
\hline$C(25)-C(26)-C(27)$ & $120.4(2)$ \\
\hline $\mathrm{C}(25)-\mathrm{C}(26)-\mathrm{H}(26)$ & 119.8 \\
\hline $\mathrm{C}(27)-\mathrm{C}(26)-\mathrm{H}(26)$ & 119.8 \\
\hline$C(28)-C(27)-C(26)$ & $120.3(3)$ \\
\hline $\mathrm{C}(28)-\mathrm{C}(27)-\mathrm{H}(27)$ & 119.9 \\
\hline $\mathrm{C}(26)-\mathrm{C}(27)-\mathrm{H}(27)$ & 119.9 \\
\hline$C(27)-C(28)-C(29)$ & $119.9(3)$ \\
\hline $\mathrm{C}(27)-\mathrm{C}(28)-\mathrm{H}(28)$ & 120.0 \\
\hline $\mathrm{C}(29)-\mathrm{C}(28)-\mathrm{H}(28)$ & 120.0 \\
\hline$C(30)-C(29)-C(28)$ & $119.7(3)$ \\
\hline $\mathrm{C}(30)-\mathrm{C}(29)-\mathrm{H}(29)$ & 120.1 \\
\hline $\mathrm{C}(28)-\mathrm{C}(29)-\mathrm{H}(29)$ & 120.1 \\
\hline$C(29)-C(30)-C(25)$ & $121.2(2)$ \\
\hline $\mathrm{C}(29)-\mathrm{C}(30)-\mathrm{H}(30)$ & 119.4 \\
\hline$C(25)-C(30)-H(30)$ & 119.4 \\
\hline$C(32)-C(31)-C(36)$ & $118.0(2)$ \\
\hline$C(32)-C(31)-P(2)$ & $117.84(18)$ \\
\hline$C(36)-C(31)-P(2)$ & $124.1(2)$ \\
\hline$C(33)-C(32)-C(31)$ & $121.3(3)$ \\
\hline $\mathrm{C}(33)-\mathrm{C}(32)-\mathrm{H}(32)$ & 119.3 \\
\hline $\mathrm{C}(31)-\mathrm{C}(32)-\mathrm{H}(32)$ & 119.3 \\
\hline$C(34)-C(33)-C(32)$ & $119.7(3)$ \\
\hline $\mathrm{C}(34)-\mathrm{C}(33)-\mathrm{H}(33)$ & 120.1 \\
\hline $\mathrm{C}(32)-\mathrm{C}(33)-\mathrm{H}(33)$ & 120.1 \\
\hline$C(35)-C(34)-C(33)$ & $119.7(3)$ \\
\hline $\mathrm{C}(35)-\mathrm{C}(34)-\mathrm{H}(34)$ & 120.1 \\
\hline $\mathrm{C}(33)-\mathrm{C}(34)-\mathrm{H}(34)$ & 120.1 \\
\hline$C(34)-C(35)-C(36)$ & $120.8(3)$ \\
\hline $\mathrm{C}(34)-\mathrm{C}(35)-\mathrm{H}(35)$ & 119.6 \\
\hline $\mathrm{C}(36)-\mathrm{C}(35)-\mathrm{H}(35)$ & 119.6 \\
\hline$C(35)-C(36)-C(31)$ & $120.3(3)$ \\
\hline $\mathrm{C}(35)-\mathrm{C}(36)-\mathrm{H}(36)$ & 119.8 \\
\hline $\mathrm{C}(31)-\mathrm{C}(36)-\mathrm{H}(36)$ & 119.8 \\
\hline $\mathrm{N}(1)-\mathrm{C}(37)-\mathrm{C}(38)$ & $109.6(2)$ \\
\hline $\mathrm{N}(1)-\mathrm{C}(37)-\mathrm{C}(46)$ & $120.9(2)$ \\
\hline$C(38)-C(37)-C(46)$ & $128.7(2)$ \\
\hline$C(39)-C(38)-C(37)$ & $105.4(2)$ \\
\hline $\mathrm{C}(39)-\mathrm{C}(38)-\mathrm{H}(38)$ & 127.3 \\
\hline $\mathrm{C}(37)-\mathrm{C}(38)-\mathrm{H}(38)$ & 127.3 \\
\hline
\end{tabular}




\begin{tabular}{|c|c|}
\hline$N(2)-C(39)-C(38)$ & $108.7(2)$ \\
\hline $\mathrm{N}(2)-\mathrm{C}(39)-\mathrm{H}(39)$ & 125.7 \\
\hline $\mathrm{C}(38)-\mathrm{C}(39)-\mathrm{H}(39)$ & 125.7 \\
\hline$N(4)-C(40)-C(41)$ & $109.2(2)$ \\
\hline$N(4)-C(40)-C(52)$ & $123.6(2)$ \\
\hline$C(41)-C(40)-C(52)$ & $127.1(2)$ \\
\hline$C(42)-C(41)-C(40)$ & $105.6(2)$ \\
\hline $\mathrm{C}(42)-\mathrm{C}(41)-\mathrm{H}(41)$ & 127.2 \\
\hline $\mathrm{C}(40)-\mathrm{C}(41)-\mathrm{H}(41)$ & 127.2 \\
\hline $\mathrm{N}(3)-\mathrm{C}(42)-\mathrm{C}(41)$ & $108.8(2)$ \\
\hline $\mathrm{N}(3)-\mathrm{C}(42)-\mathrm{H}(42)$ & 125.6 \\
\hline $\mathrm{C}(41)-\mathrm{C}(42)-\mathrm{H}(42)$ & 125.6 \\
\hline$N(6)-C(43)-C(44)$ & $110.9(2)$ \\
\hline$N(6)-C(43)-C(58)$ & $120.2(2)$ \\
\hline$C(44)-C(43)-C(58)$ & $128.9(2)$ \\
\hline$C(45)-C(44)-C(43)$ & $104.6(2)$ \\
\hline $\mathrm{C}(45)-\mathrm{C}(44)-\mathrm{H}(44)$ & 127.7 \\
\hline $\mathrm{C}(43)-\mathrm{C}(44)-\mathrm{H}(44)$ & 127.7 \\
\hline $\mathrm{N}(5)-\mathrm{C}(45)-\mathrm{C}(44)$ & $108.5(2)$ \\
\hline $\mathrm{N}(5)-\mathrm{C}(45)-\mathrm{H}(45)$ & 125.7 \\
\hline $\mathrm{C}(44)-\mathrm{C}(45)-\mathrm{H}(45)$ & 125.7 \\
\hline$C(47)-C(46)-C(51)$ & $118.7(2)$ \\
\hline$C(47)-C(46)-C(37)$ & $123.1(2)$ \\
\hline$C(51)-C(46)-C(37)$ & $118.0(2)$ \\
\hline$C(48)-C(47)-C(46)$ & $120.0(3)$ \\
\hline $\mathrm{C}(48)-\mathrm{C}(47)-\mathrm{H}(47)$ & 120.0 \\
\hline $\mathrm{C}(46)-\mathrm{C}(47)-\mathrm{H}(47)$ & 120.0 \\
\hline$C(49)-C(48)-C(47)$ & $120.7(3)$ \\
\hline $\mathrm{C}(49)-\mathrm{C}(48)-\mathrm{H}(48)$ & 119.7 \\
\hline $\mathrm{C}(47)-\mathrm{C}(48)-\mathrm{H}(48)$ & 119.7 \\
\hline$C(48)-C(49)-C(50)$ & $120.2(3)$ \\
\hline $\mathrm{C}(48)-\mathrm{C}(49)-\mathrm{H}(49)$ & 119.9 \\
\hline $\mathrm{C}(50)-\mathrm{C}(49)-\mathrm{H}(49)$ & 119.9 \\
\hline$C(49)-C(50)-C(51)$ & $119.6(3)$ \\
\hline $\mathrm{C}(49)-\mathrm{C}(50)-\mathrm{H}(50)$ & 120.2 \\
\hline $\mathrm{C}(51)-\mathrm{C}(50)-\mathrm{H}(50)$ & 120.2 \\
\hline$C(50)-C(51)-C(46)$ & $120.7(2)$ \\
\hline $\mathrm{C}(50)-\mathrm{C}(51)-\mathrm{H}(51)$ & 119.6 \\
\hline $\mathrm{C}(46)-\mathrm{C}(51)-\mathrm{H}(51)$ & 119.6 \\
\hline$C(53)-C(52)-C(57)$ & $118.2(2)$ \\
\hline$C(53)-C(52)-C(40)$ & $121.7(2)$ \\
\hline$C(57)-C(52)-C(40)$ & $120.0(2)$ \\
\hline$C(54)-C(53)-C(52)$ & $120.6(2)$ \\
\hline $\mathrm{C}(54)-\mathrm{C}(53)-\mathrm{H}(53)$ & 119.7 \\
\hline $\mathrm{C}(52)-\mathrm{C}(53)-\mathrm{H}(53)$ & 119.7 \\
\hline$C(55)-C(54)-C(53)$ & $120.6(3)$ \\
\hline $\mathrm{C}(55)-\mathrm{C}(54)-\mathrm{H}(54)$ & 119.7 \\
\hline $\mathrm{C}(53)-\mathrm{C}(54)-\mathrm{H}(54)$ & 119.7 \\
\hline$C(54)-C(55)-C(56)$ & $119.2(3)$ \\
\hline $\mathrm{C}(54)-\mathrm{C}(55)-\mathrm{H}(55)$ & 120.4 \\
\hline $\mathrm{C}(56)-\mathrm{C}(55)-\mathrm{H}(55)$ & 120.4 \\
\hline$C(57)-C(56)-C(55)$ & $120.6(3)$ \\
\hline $\mathrm{C}(57)-\mathrm{C}(56)-\mathrm{H}(56)$ & 119.7 \\
\hline $\mathrm{C}(55)-\mathrm{C}(56)-\mathrm{H}(56)$ & 119.7 \\
\hline$C(56)-C(57)-C(52)$ & $120.8(3)$ \\
\hline $\mathrm{C}(56)-\mathrm{C}(57)-\mathrm{H}(57)$ & 119.6 \\
\hline $\mathrm{C}(52)-\mathrm{C}(57)-\mathrm{H}(57)$ & 119.6 \\
\hline
\end{tabular}




\begin{tabular}{|c|c|}
\hline$C(63)-C(58)-C(59)$ & $118.0(2)$ \\
\hline$C(63)-C(58)-C(43)$ & $120.9(2)$ \\
\hline$C(59)-C(58)-C(43)$ & $121.1(2)$ \\
\hline$C(60)-C(59)-C(58)$ & $120.9(3)$ \\
\hline $\mathrm{C}(60)-\mathrm{C}(59)-\mathrm{H}(59)$ & 119.6 \\
\hline $\mathrm{C}(58)-\mathrm{C}(59)-\mathrm{H}(59)$ & 119.6 \\
\hline$C(61)-C(60)-C(59)$ & $120.6(3)$ \\
\hline $\mathrm{C}(61)-\mathrm{C}(60)-\mathrm{H}(60)$ & 119.7 \\
\hline $\mathrm{C}(59)-\mathrm{C}(60)-\mathrm{H}(60)$ & 119.7 \\
\hline$C(60)-C(61)-C(62)$ & $119.4(3)$ \\
\hline $\mathrm{C}(60)-\mathrm{C}(61)-\mathrm{H}(61)$ & 120.3 \\
\hline $\mathrm{C}(62)-\mathrm{C}(61)-\mathrm{H}(61)$ & 120.3 \\
\hline$C(61)-C(62)-C(63)$ & $119.9(3)$ \\
\hline $\mathrm{C}(61)-\mathrm{C}(62)-\mathrm{H}(62)$ & 120.0 \\
\hline $\mathrm{C}(63)-\mathrm{C}(62)-\mathrm{H}(62)$ & 120.0 \\
\hline$C(58)-C(63)-C(62)$ & $121.1(3)$ \\
\hline $\mathrm{C}(58)-\mathrm{C}(63)-\mathrm{H}(63)$ & 119.4 \\
\hline $\mathrm{C}(62)-\mathrm{C}(63)-\mathrm{H}(63)$ & 119.4 \\
\hline$C(66)-C(65)-C(64)$ & $113.3(6)$ \\
\hline $\mathrm{C}(66)-\mathrm{C}(65)-\mathrm{H}(65 \mathrm{~A})$ & 108.9 \\
\hline $\mathrm{C}(64)-\mathrm{C}(65)-\mathrm{H}(65 \mathrm{~A})$ & 108.9 \\
\hline $\mathrm{C}(66)-\mathrm{C}(65)-\mathrm{H}(65 \mathrm{~B})$ & 108.9 \\
\hline $\mathrm{C}(64)-\mathrm{C}(65)-\mathrm{H}(65 \mathrm{~B})$ & 108.9 \\
\hline $\mathrm{H}(65 \mathrm{~A})-\mathrm{C}(65)-\mathrm{H}(65 \mathrm{~B})$ & 107.7 \\
\hline$C(65)-C(66)-C(67)$ & $100.2(6)$ \\
\hline $\mathrm{C}(65)-\mathrm{C}(66)-\mathrm{H}(66 \mathrm{~A})$ & 111.7 \\
\hline $\mathrm{C}(67)-\mathrm{C}(66)-\mathrm{H}(66 \mathrm{~A})$ & 111.7 \\
\hline $\mathrm{C}(65)-\mathrm{C}(66)-\mathrm{H}(66 \mathrm{~B})$ & 111.7 \\
\hline $\mathrm{C}(67)-\mathrm{C}(66)-\mathrm{H}(66 \mathrm{~B})$ & 111.7 \\
\hline $\mathrm{H}(66 \mathrm{~A})-\mathrm{C}(66)-\mathrm{H}(66 \mathrm{~B})$ & 109.5 \\
\hline $\mathrm{C}(65)-\mathrm{C}(66)-\mathrm{H}(67 \mathrm{C})$ & $145.8(8)$ \\
\hline $\mathrm{C}(67)-\mathrm{C}(66)-\mathrm{H}(67 \mathrm{C})$ & $100.6(4)$ \\
\hline $\mathrm{H}(66 \mathrm{~A})-\mathrm{C}(66)-\mathrm{H}(67 \mathrm{C})$ & 34.6 \\
\hline $\mathrm{H}(66 \mathrm{~B})-\mathrm{C}(66)-\mathrm{H}(67 \mathrm{C})$ & 85.0 \\
\hline$C(68)-C(67)-C(66)$ & $100.9(6)$ \\
\hline$C(68)-C(67)-H(67 A)$ & 111.6 \\
\hline $\mathrm{C}(66)-\mathrm{C}(67)-\mathrm{H}(67 \mathrm{~A})$ & 111.6 \\
\hline $\mathrm{C}(68)-\mathrm{C}(67)-\mathrm{H}(67 \mathrm{~B})$ & 111.6 \\
\hline $\mathrm{C}(66)-\mathrm{C}(67)-\mathrm{H}(67 \mathrm{~B})$ & 111.6 \\
\hline $\mathrm{H}(67 \mathrm{~A})-\mathrm{C}(67)-\mathrm{H}(67 \mathrm{~B})$ & 109.4 \\
\hline $\mathrm{N}(5)-\mathrm{B}(1)-\mathrm{N}(3)$ & $110.0(2)$ \\
\hline $\mathrm{N}(5)-\mathrm{B}(1)-\mathrm{N}(2)$ & $111.6(2)$ \\
\hline $\mathrm{N}(3)-\mathrm{B}(1)-\mathrm{N}(2)$ & $104.97(19)$ \\
\hline $\mathrm{N}(5)-\mathrm{B}(1)-\mathrm{H}(1 \mathrm{~B})$ & $108.3(13)$ \\
\hline $\mathrm{N}(3)-\mathrm{B}(1)-\mathrm{H}(1 \mathrm{~B})$ & $107.4(12)$ \\
\hline $\mathrm{N}(2)-\mathrm{B}(1)-\mathrm{H}(1 \mathrm{~B})$ & $114.4(13)$ \\
\hline $\mathrm{C}(39)-\mathrm{N}(2)-\mathrm{N}(1)$ & $109.26(19)$ \\
\hline $\mathrm{C}(39)-\mathrm{N}(2)-\mathrm{B}(1)$ & $132.7(2)$ \\
\hline $\mathrm{N}(1)-\mathrm{N}(2)-\mathrm{B}(1)$ & $117.08(18)$ \\
\hline $\mathrm{C}(37)-\mathrm{N}(1)-\mathrm{N}(2)$ & $107.05(18)$ \\
\hline $\mathrm{C}(37)-\mathrm{N}(1)-\mathrm{Rh}(1)$ & $138.84(16)$ \\
\hline $\mathrm{N}(2)-\mathrm{N}(1)-\mathrm{Rh}(1)$ & $113.28(14)$ \\
\hline $\mathrm{C}(45)-\mathrm{N}(5)-\mathrm{N}(6)$ & $110.3(2)$ \\
\hline $\mathrm{C}(45)-\mathrm{N}(5)-\mathrm{B}(1)$ & $125.0(2)$ \\
\hline $\mathrm{N}(6)-\mathrm{N}(5)-\mathrm{B}(1)$ & $124.4(2)$ \\
\hline $\mathrm{C}(43)-\mathrm{N}(6)-\mathrm{N}(5)$ & $105.7(2)$ \\
\hline $\mathrm{C}(42)-\mathrm{N}(3)-\mathrm{N}(4)$ & $109.35(19)$ \\
\hline
\end{tabular}




$$
\begin{aligned}
& \mathrm{C}(42)-\mathrm{N}(3)-\mathrm{B}(1) \quad 130.3(2) \\
& \mathrm{N}(4)-\mathrm{N}(3)-\mathrm{B}(1) \quad 119.14(18) \\
& \mathrm{C}(40)-\mathrm{N}(4)-\mathrm{N}(3) \quad 107.03(18) \\
& \mathrm{C}(40)-\mathrm{N}(4)-\mathrm{Rh}(1) \quad 138.44(16) \\
& \mathrm{N}(3)-\mathrm{N}(4)-\mathrm{Rh}(1) \quad 113.85(14) \\
& \mathrm{C}(13)-\mathrm{P}(1)-\mathrm{C}(7) \quad 107.10(10) \\
& \mathrm{C}(13)-\mathrm{P}(1)-\mathrm{C}(1) \quad 97.75(10) \\
& \mathrm{C}(7)-\mathrm{P}(1)-\mathrm{C}(1) \quad 100.58(10) \\
& \mathrm{C}(13)-\mathrm{P}(1)-\mathrm{Rh}(1) \quad 113.06(7) \\
& \mathrm{C}(7)-\mathrm{P}(1)-\mathrm{Rh}(1) \quad 119.04(7) \\
& \mathrm{C}(1)-\mathrm{P}(1)-\mathrm{Rh}(1) \quad 116.60(7) \\
& \mathrm{C}(25)-\mathrm{P}(2)-\mathrm{C}(31) \quad 104.17(11) \\
& \mathrm{C}(25)-\mathrm{P}(2)-\mathrm{C}(19) \quad 99.55(11) \\
& \mathrm{C}(31)-\mathrm{P}(2)-\mathrm{C}(19) \quad 101.89(11) \\
& \mathrm{C}(25)-\mathrm{P}(2)-\mathrm{Rh}(1) \quad 113.04(8) \\
& \mathrm{C}(31)-\mathrm{P}(2)-\mathrm{Rh}(1) \quad 115.42(8) \\
& \mathrm{C}(19)-\mathrm{P}(2)-\mathrm{Rh}(1) \quad 120.40(7) \\
& \mathrm{N}(4)-\mathrm{Rh}(1)-\mathrm{N}(1) \quad 78.98(7) \\
& \mathrm{N}(4)-\mathrm{Rh}(1)-\mathrm{P}(1) \quad 91.82(5) \\
& \mathrm{N}(1)-\mathrm{Rh}(1)-\mathrm{P}(1) \quad 167.28(5) \\
& \mathrm{N}(4)-\mathrm{Rh}(1)-\mathrm{P}(2) \quad 171.38(5) \\
& \mathrm{N}(1)-\mathrm{Rh}(1)-\mathrm{P}(2) \quad 92.54(5) \\
& \mathrm{P}(1)-\mathrm{Rh}(1)-\mathrm{P}(2) \quad 96.32(2) \\
& \mathrm{C}(66 \mathrm{~B})-\mathrm{C}(67 \mathrm{~B})-\mathrm{H}(67 \mathrm{C}) \quad 113.1(10) \\
& \mathrm{C}(66 \mathrm{~B})-\mathrm{C}(67 \mathrm{~B})-\mathrm{H}(67 \mathrm{D}) \quad 110.1(10) \\
& \mathrm{H}(67 \mathrm{C})-\mathrm{C}(67 \mathrm{~B})-\mathrm{H}(67 \mathrm{D}) \quad 111.4(10) \\
& \mathrm{C}(67 \mathrm{~B})-\mathrm{C}(66 \mathrm{~B})-\mathrm{C}(65 \mathrm{~B}) \quad 96.0(7) \\
& \mathrm{C}(67 \mathrm{~B})-\mathrm{C}(66 \mathrm{~B})-\mathrm{H}(66 \mathrm{C}) \quad 112.5 \\
& \mathrm{C}(65 \mathrm{~B})-\mathrm{C}(66 \mathrm{~B})-\mathrm{H}(66 \mathrm{C}) \quad 112.5 \\
& \mathrm{C}(67 \mathrm{~B})-\mathrm{C}(66 \mathrm{~B})-\mathrm{H}(66 \mathrm{D}) \quad 112.6 \\
& \mathrm{C}(65 \mathrm{~B})-\mathrm{C}(66 \mathrm{~B})-\mathrm{H}(66 \mathrm{D}) \quad 112.6 \\
& \mathrm{H}(66 \mathrm{C})-\mathrm{C}(66 \mathrm{~B})-\mathrm{H}(66 \mathrm{D}) \quad 110.1 \\
& C(66 B)-C(65 B)-C(64 B) \quad 93.2(7) \\
& C(66 B)-C(65 B)-H(65 C) \quad 113.1 \\
& C(64 B)-C(65 B)-H(65 C) \quad 113.0 \\
& C(66 B)-C(65 B)-H(65 D) \quad 113.1 \\
& C(64 B)-C(65 B)-H(65 D) \quad 113.0 \\
& \mathrm{H}(65 \mathrm{C})-\mathrm{C}(65 \mathrm{~B})-\mathrm{H}(65 \mathrm{D}) \quad 110.5 \\
& C(65 B)-C(64 B)-H(64 D) \quad 109.5 \\
& C(65 B)-C(64 B)-H(64 E) \quad 109.4 \\
& \mathrm{H}(64 \mathrm{D})-\mathrm{C}(64 \mathrm{~B})-\mathrm{H}(64 \mathrm{E}) \quad 109.5 \\
& \mathrm{C}(65 \mathrm{~B})-\mathrm{C}(64 \mathrm{~B})-\mathrm{H}(64 \mathrm{~F}) \quad 109.4 \\
& \mathrm{H}(64 \mathrm{D})-\mathrm{C}(64 \mathrm{~B})-\mathrm{H}(64 \mathrm{~F}) \quad 109.5 \\
& \mathrm{H}(64 \mathrm{E})-\mathrm{C}(64 \mathrm{~B})-\mathrm{H}(64 \mathrm{~F}) \quad 109.5
\end{aligned}
$$

Symmetry transformations used to generate equivalent atoms: 
Table S16. Anisotropic displacement parameters ( $A^{\wedge} 2 \times 10^{\wedge} 3$ ) for complex 4. The anisotropic displacement factor exponent takes the form:

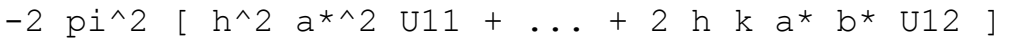

\begin{tabular}{|c|c|c|c|c|c|c|}
\hline & $\mathrm{U} 11$ & $\mathrm{U} 22$ & U33 & U23 & U13 & $\mathrm{U} 12$ \\
\hline$C(1)$ & $18(1)$ & $18(1)$ & $21(1)$ & $3(1)$ & $6(1)$ & $-2(1)$ \\
\hline$C(2)$ & $26(1)$ & $26(1)$ & $26(1)$ & $3(1)$ & $10(1)$ & $2(1)$ \\
\hline$C(3)$ & $33(1)$ & $39(2)$ & $22(1)$ & $7(1)$ & $6(1)$ & $-1(1)$ \\
\hline$C(4)$ & $28(1)$ & $43(2)$ & $37(2)$ & $14(1)$ & $4(1)$ & $10(1)$ \\
\hline$C(5)$ & $29(1)$ & $39(2)$ & $42(2)$ & $8(1)$ & $13(1)$ & $13(1)$ \\
\hline$C(6)$ & $26(1)$ & $29(1)$ & $29(1)$ & $-1(1)$ & $10(1)$ & $5(1)$ \\
\hline$C(7)$ & $22(1)$ & $24(1)$ & $19(1)$ & $-1(1)$ & $9(1)$ & $-1(1)$ \\
\hline$C(8)$ & $34(1)$ & $25(1)$ & $26(1)$ & $-4(1)$ & $15(1)$ & $-8(1)$ \\
\hline$C(9)$ & $56(2)$ & $35(2)$ & $33(2)$ & $-16(1)$ & $26(1)$ & $-16(1)$ \\
\hline$C(10)$ & $60(2)$ & $55(2)$ & $36(2)$ & $-15(1)$ & $36(2)$ & $-20(2)$ \\
\hline$C(11)$ & $55(2)$ & $52(2)$ & $38(2)$ & $-14(1)$ & $29(1)$ & $-29(2)$ \\
\hline$C(12)$ & $39(1)$ & $34(2)$ & $26(1)$ & $-9(1)$ & $16(1)$ & $-16(1)$ \\
\hline$C(13)$ & $22(1)$ & $17(1)$ & $15(1)$ & $0(1)$ & $6(1)$ & $-1(1)$ \\
\hline$C(14)$ & $21(1)$ & $20(1)$ & $21(1)$ & $0(1)$ & $5(1)$ & $2(1)$ \\
\hline$C(15)$ & $28(1)$ & $29(1)$ & $33(1)$ & $-6(1)$ & $6(1)$ & $8(1)$ \\
\hline$C(16)$ & $38(1)$ & $22(1)$ & $27(1)$ & $-8(1)$ & $4(1)$ & $3(1)$ \\
\hline$C(17)$ & $28(1)$ & $27(1)$ & $21(1)$ & $-3(1)$ & $4(1)$ & $-6(1)$ \\
\hline$C(18)$ & $20(1)$ & $26(1)$ & $21(1)$ & $-1(1)$ & $7(1)$ & $-1(1)$ \\
\hline$C(19)$ & $29(1)$ & $22(1)$ & $19(1)$ & $5(1)$ & $-2(1)$ & $-4(1)$ \\
\hline$C(20)$ & $35(1)$ & $26(1)$ & $31(1)$ & $3(1)$ & $1(1)$ & $-3(1)$ \\
\hline$C(21)$ & $54(2)$ & $23(1)$ & $37(2)$ & $2(1)$ & $-4(1)$ & $-2(1)$ \\
\hline$C(22)$ & $60(2)$ & $25(2)$ & $39(2)$ & $9(1)$ & $-6(1)$ & $-17(1)$ \\
\hline$C(23)$ & $46(2)$ & $39(2)$ & $39(2)$ & $12(1)$ & $4(1)$ & $-19(1)$ \\
\hline$C(24)$ & $33(1)$ & $30(1)$ & $30(1)$ & $9(1)$ & $3(1)$ & $-7(1)$ \\
\hline$C(25)$ & $23(1)$ & $19(1)$ & $23(1)$ & $4(1)$ & $6(1)$ & $1(1)$ \\
\hline$C(26)$ & $26(1)$ & $32(1)$ & $25(1)$ & $-1(1)$ & $4(1)$ & $-1(1)$ \\
\hline$C(27)$ & $26(1)$ & $46(2)$ & $37(2)$ & $1(1)$ & $1(1)$ & $1(1)$ \\
\hline$C(28)$ & $26(1)$ & $49(2)$ & $55(2)$ & $5(2)$ & $11(1)$ & $9(1)$ \\
\hline$C(29)$ & $40(2)$ & $39(2)$ & $40(2)$ & $6(1)$ & $20(1)$ & $12(1)$ \\
\hline$C(30)$ & $33(1)$ & $25(1)$ & $26(1)$ & $3(1)$ & $8(1)$ & $5(1)$ \\
\hline$C(31)$ & $21(1)$ & $28(1)$ & $18(1)$ & $-2(1)$ & $6(1)$ & $-6(1)$ \\
\hline$C(32)$ & $32(1)$ & $31(1)$ & $27(1)$ & $-4(1)$ & $13(1)$ & $-7(1)$ \\
\hline C (33) & $37(1)$ & $37(2)$ & $36(2)$ & $-13(1)$ & $13(1)$ & $-6(1)$ \\
\hline$C(34)$ & $35(1)$ & $61(2)$ & $27(1)$ & $-16(1)$ & $15(1)$ & $-9(1)$ \\
\hline$C(35)$ & $38(1)$ & $57(2)$ & $19(1)$ & $2(1)$ & $10(1)$ & $-8(1)$ \\
\hline$C(36)$ & $30(1)$ & $37(2)$ & $23(1)$ & $3(1)$ & $7(1)$ & $-5(1)$ \\
\hline$C(37)$ & $21(1)$ & $21(1)$ & $26(1)$ & $4(1)$ & $7(1)$ & $-1(1)$ \\
\hline$C(38)$ & $21(1)$ & $31(1)$ & $39(2)$ & $1(1)$ & $12(1)$ & $-4(1)$ \\
\hline$C(39)$ & $23(1)$ & $28(1)$ & $35(1)$ & $2(1)$ & $16(1)$ & $1(1)$ \\
\hline$C(40)$ & $21(1)$ & $22(1)$ & $24(1)$ & $5(1)$ & $7(1)$ & $0(1)$ \\
\hline$C(41)$ & $36(1)$ & $32(2)$ & $26(1)$ & $12(1)$ & $11(1)$ & $8(1)$ \\
\hline$C(42)$ & $35(1)$ & $38(2)$ & $20(1)$ & $9(1)$ & $12(1)$ & $3(1)$ \\
\hline$C(43)$ & $27(1)$ & $27(1)$ & $23(1)$ & $0(1)$ & $11(1)$ & $7(1)$ \\
\hline$C(44)$ & $39(1)$ & $32(2)$ & $28(1)$ & $-10(1)$ & $15(1)$ & $-2(1)$ \\
\hline$C(45)$ & $36(1)$ & $24(1)$ & $35(1)$ & $-4(1)$ & $16(1)$ & $-2(1)$ \\
\hline$C(46)$ & $26(1)$ & $20(1)$ & $28(1)$ & $2(1)$ & $10(1)$ & $-7(1)$ \\
\hline$C(47)$ & $35(1)$ & $24(1)$ & $34(1)$ & $4(1)$ & $4(1)$ & $-2(1)$ \\
\hline
\end{tabular}




\begin{tabular}{|c|c|c|c|c|c|c|}
\hline$C(48)$ & $55(2)$ & $32(2)$ & $30(2)$ & $-2(1)$ & $1(1)$ & $-7(1)$ \\
\hline$C(49)$ & $71(2)$ & $23(2)$ & $35(2)$ & $-6(1)$ & $19(2)$ & $-7(1)$ \\
\hline$C(50)$ & $41(2)$ & $25(1)$ & $45(2)$ & $-2(1)$ & $17(1)$ & $-2(1)$ \\
\hline$C(51)$ & $27(1)$ & $26(1)$ & $33(1)$ & $1(1)$ & $8(1)$ & $-4(1)$ \\
\hline$C(52)$ & $21(1)$ & $19(1)$ & $33(1)$ & $2(1)$ & $11(1)$ & $-1(1)$ \\
\hline C (53) & $31(1)$ & $22(1)$ & $32(1)$ & $-1(1)$ & $14(1)$ & $-1(1)$ \\
\hline$C(54)$ & $52(2)$ & $32(2)$ & $41(2)$ & $-6(1)$ & $27(1)$ & $-1(1)$ \\
\hline$C(55)$ & $51(2)$ & $32(2)$ & $72(2)$ & $-6(2)$ & $40(2)$ & $5(1)$ \\
\hline$C(56)$ & $35(2)$ & $35(2)$ & $72(2)$ & $6(2)$ & $24(2)$ & $13(1)$ \\
\hline C (57) & $28(1)$ & $32(2)$ & $45(2)$ & $8(1)$ & $11(1)$ & $4(1)$ \\
\hline$C(58)$ & $29(1)$ & $26(1)$ & $24(1)$ & $4(1)$ & $13(1)$ & $11(1)$ \\
\hline C (59) & 35 (1) & $37(2)$ & $24(1)$ & $-1(1)$ & $11(1)$ & $12(1)$ \\
\hline$C(60)$ & $42(2)$ & $51(2)$ & $25(1)$ & $7(1)$ & $18(1)$ & $19(1)$ \\
\hline$C(61)$ & $41(2)$ & $51(2)$ & $38(2)$ & $19(1)$ & $23(1)$ & $13(1)$ \\
\hline$C(62)$ & $37(2)$ & $41(2)$ & $43(2)$ & $7(1)$ & $17(1)$ & $1(1)$ \\
\hline$C(63)$ & $36(1)$ & $35(2)$ & $27(1)$ & $1(1)$ & $12(1)$ & $4(1)$ \\
\hline B (1) & $28(1)$ & $24(2)$ & $23(1)$ & $0(1)$ & $11(1)$ & $-1(1)$ \\
\hline $\mathrm{N}(2)$ & $24(1)$ & $24(1)$ & $23(1)$ & $2(1)$ & $11(1)$ & $1(1)$ \\
\hline$N(1)$ & $21(1)$ & $21(1)$ & $19(1)$ & $1(1)$ & $8(1)$ & $-1(1)$ \\
\hline$N(5)$ & $32(1)$ & $22(1)$ & $24(1)$ & $-1(1)$ & $14(1)$ & $0(1)$ \\
\hline$N(6)$ & $35(1)$ & $24(1)$ & $25(1)$ & $-1(1)$ & $16(1)$ & $-1(1)$ \\
\hline $\mathrm{N}(3)$ & $24(1)$ & $24(1)$ & $17(1)$ & $1(1)$ & $9(1)$ & $0(1)$ \\
\hline $\mathrm{N}(4)$ & $20(1)$ & $21(1)$ & $17(1)$ & $1(1)$ & $7(1)$ & $0(1)$ \\
\hline P (1) & $18(1)$ & $17(1)$ & $16(1)$ & $-1(1)$ & $6(1)$ & $0(1)$ \\
\hline$P(2)$ & $20(1)$ & $19(1)$ & $16(1)$ & $1(1)$ & $4(1)$ & $-2(1)$ \\
\hline $\mathrm{Rh}(1)$ & $17(1)$ & $16(1)$ & $15(1)$ & $1(1)$ & $6(1)$ & $-1(1)$ \\
\hline
\end{tabular}


Table S17. Hydrogen coordinates $\left(x 1^{\wedge} 4\right)$ and isotropic displacement parameters $\left(A^{\wedge} 2 \times 10^{\wedge} 3\right)$ for complex 4.

\begin{tabular}{|c|c|c|c|c|}
\hline & $x$ & y & $z$ & $\mathrm{U}(\mathrm{eq})$ \\
\hline $\mathrm{H}(2)$ & 5264 & 1656 & 8976 & 30 \\
\hline $\mathrm{H}(3)$ & 6357 & 1194 & 9930 & 38 \\
\hline $\mathrm{H}(4)$ & 7310 & 486 & 9617 & 45 \\
\hline $\mathrm{H}(5)$ & 7173 & 247 & 8352 & 44 \\
\hline $\mathrm{H}(6)$ & 6113 & 750 & 7392 & 33 \\
\hline $\mathrm{H}(8)$ & 4592 & 774 & 6138 & 33 \\
\hline $\mathrm{H}(9)$ & 5046 & 698 & 5086 & 47 \\
\hline $\mathrm{H}(10)$ & 5884 & 1591 & 4865 & 55 \\
\hline $\mathrm{H}(11)$ & 6255 & 2559 & 5693 & 54 \\
\hline $\mathrm{H}(12)$ & 5807 & 2642 & 6749 & 38 \\
\hline $\mathrm{H}(14)$ & 3726 & 2759 & 7679 & 25 \\
\hline $\mathrm{H}(15)$ & 3869 & 3874 & 8284 & 36 \\
\hline $\mathrm{H}(16)$ & 5134 & 4404 & 8750 & 36 \\
\hline $\mathrm{H}(17)$ & 6258 & 3815 & 8615 & 32 \\
\hline $\mathrm{H}(18)$ & 6124 & 2691 & 8027 & 27 \\
\hline $\mathrm{H}(20)$ & 2358 & 3386 & 6448 & 39 \\
\hline $\mathrm{H}(21)$ & 2881 & 4549 & 6495 & 50 \\
\hline $\mathrm{H}(22)$ & 4060 & 4736 & 6194 & 55 \\
\hline $\mathrm{H}(23)$ & 4661 & 3789 & 5758 & 52 \\
\hline $\mathrm{H}(24)$ & 4129 & 2624 & 5685 & 39 \\
\hline $\mathrm{H}(26)$ & 1368 & 1851 & 4930 & 34 \\
\hline $\mathrm{H}(27)$ & 34 & 2119 & 4819 & 46 \\
\hline $\mathrm{H}(28)$ & -305 & 2548 & 5859 & 52 \\
\hline $\mathrm{H}(29)$ & 687 & 2679 & 7031 & 45 \\
\hline $\mathrm{H}(30)$ & 2008 & 2375 & 7153 & 34 \\
\hline $\mathrm{H}(32)$ & 3047 & 528 & 5656 & 35 \\
\hline $\mathrm{H}(33)$ & 3121 & -17 & 4547 & 43 \\
\hline $\mathrm{H}(34)$ & 3069 & 690 & 3492 & 47 \\
\hline $\mathrm{H}(35)$ & 2868 & 1926 & 3528 & 45 \\
\hline $\mathrm{H}(36)$ & 2738 & 2471 & 4614 & 36 \\
\hline $\mathrm{H}(38)$ & 617 & 447 & 7070 & 36 \\
\hline $\mathrm{H}(39)$ & 1241 & 1129 & 8273 & 32 \\
\hline $\mathrm{H}(41)$ & 4537 & -589 & 9413 & 37 \\
\hline $\mathrm{H}(42)$ & 3695 & 347 & 9766 & 36 \\
\hline $\mathrm{H}(44)$ & 2830 & 2396 & 10814 & 38 \\
\hline $\mathrm{H}(45)$ & 3292 & 2565 & 9674 & 37 \\
\hline $\mathrm{H}(47)$ & 748 & 453 & 5473 & 39 \\
\hline $\mathrm{H}(48)$ & 776 & -373 & 4534 & 50 \\
\hline $\mathrm{H}(49)$ & 1802 & -1207 & 4743 & 51 \\
\hline $\mathrm{H}(50)$ & 2808 & -1247 & 5910 & 43 \\
\hline $\mathrm{H}(51)$ & 2783 & -435 & 6862 & 35 \\
\hline $\mathrm{H}(53)$ & 4059 & 16 & 6907 & 33 \\
\hline $\mathrm{H}(54)$ & 4659 & -661 & 6174 & 46 \\
\hline $\mathrm{H}(55)$ & 5666 & -1487 & 6742 & 56 \\
\hline $\mathrm{H}(56)$ & 6087 & -1616 & 8057 & 55 \\
\hline $\mathrm{H}(57)$ & 5509 & -932 & 8796 & 42 \\
\hline $\mathrm{H}(59)$ & 2434 & 1635 & 11723 & 38 \\
\hline $\mathrm{H}(60)$ & 1738 & 1092 & 12457 & 45 \\
\hline
\end{tabular}




\begin{tabular}{|c|c|c|c|c|}
\hline $\mathrm{H}(61)$ & 831 & 168 & 11963 & 49 \\
\hline $\mathrm{H}(62)$ & 620 & -215 & 10713 & 47 \\
\hline $\mathrm{H}(63)$ & 1297 & 347 & 9964 & 38 \\
\hline $\mathrm{H}(64 \mathrm{~A})$ & -480 & 7900 & 924 & 154 \\
\hline $\mathrm{H}(64 \mathrm{~B})$ & 412 & 7836 & 866 & 154 \\
\hline $\mathrm{H}(64 \mathrm{C})$ & -71 & 8580 & 657 & 154 \\
\hline $\mathrm{H}(65 \mathrm{~A})$ & 198 & 8903 & 1827 & 111 \\
\hline $\mathrm{H}(65 \mathrm{~B})$ & 116 & 8098 & 2106 & 111 \\
\hline $\mathrm{H}(66 \mathrm{~A})$ & 1484 & 8657 & 1800 & 124 \\
\hline $\mathrm{H}(66 \mathrm{~B})$ & 1401 & 7836 & 2061 & 124 \\
\hline$H(67 A)$ & 1013 & 9022 & 2943 & 127 \\
\hline $\mathrm{H}(67 \mathrm{~B})$ & 1270 & 8214 & 3250 & 127 \\
\hline$H(68 A)$ & 2405 & 8859 & 3685 & 232 \\
\hline$H(68 B)$ & 2244 & 9272 & 2894 & 232 \\
\hline $\mathrm{H}(68 \mathrm{C})$ & 2497 & 8439 & 2961 & 232 \\
\hline $\mathrm{H}(66 \mathrm{C})$ & 1680 & 7625 & 3163 & 133 \\
\hline $\mathrm{H}(66 \mathrm{D})$ & 1471 & 7315 & 2304 & 133 \\
\hline $\mathrm{H}(65 \mathrm{C})$ & 838 & 8719 & 2657 & 117 \\
\hline $\mathrm{H}(65 \mathrm{D})$ & 267 & 8019 & 2358 & 117 \\
\hline$H(64 D)$ & 412 & 8693 & 1243 & 399 \\
\hline $\mathrm{H}(64 \mathrm{E})$ & 806 & 7909 & 1282 & 399 \\
\hline $\mathrm{H}(64 \mathrm{~F})$ & 1365 & 8599 & 1576 & 399 \\
\hline$H(67 C)$ & 1914 & 8521 & 1977 & 100 \\
\hline$H(67 D)$ & 2653 & 8052 & 2513 & 100 \\
\hline $\mathrm{H}(1 \mathrm{~B})$ & $3264(14)$ & $1922(13)$ & 8464 (13) & $19(6)$ \\
\hline
\end{tabular}


Table s18. Torsion angles [deg] for complex 4.

\begin{tabular}{|c|c|}
\hline$C(6)-C(1)-C(2)-C(3)$ & $2.0(4)$ \\
\hline$P(1)-C(1)-C(2)-C(3)$ & $-175.50(19)$ \\
\hline$C(1)-C(2)-C(3)-C(4)$ & $-1.8(4)$ \\
\hline$C(2)-C(3)-C(4)-C(5)$ & $0.1(4)$ \\
\hline$C(3)-C(4)-C(5)-C(6)$ & $1.4(4)$ \\
\hline$C(2)-C(1)-C(6)-C(5)$ & $-0.4(4)$ \\
\hline$P(1)-C(1)-C(6)-C(5)$ & $176.9(2)$ \\
\hline$C(4)-C(5)-C(6)-C(1)$ & $-1.2(4)$ \\
\hline$C(12)-C(7)-C(8)-C(9)$ & $0.2(4)$ \\
\hline$P(1)-C(7)-C(8)-C(9)$ & $-179.8(2)$ \\
\hline$C(7)-C(8)-C(9)-C(10)$ & $0.0(5)$ \\
\hline$C(8)-C(9)-C(10)-C(11)$ & $-0.3(5)$ \\
\hline$C(9)-C(10)-C(11)-C(12)$ & $0.3(5)$ \\
\hline$C(10)-C(11)-C(12)-C(7)$ & $-0.1(5)$ \\
\hline$C(8)-C(7)-C(12)-C(11)$ & $-0.2(4)$ \\
\hline$P(1)-C(7)-C(12)-C(11)$ & $179.8(2)$ \\
\hline$C(18)-C(13)-C(14)-C(15)$ & $1.5(3)$ \\
\hline$P(1)-C(13)-C(14)-C(15)$ & $174.75(19)$ \\
\hline$C(13)-C(14)-C(15)-C(16)$ & $-0.3(4)$ \\
\hline$C(14)-C(15)-C(16)-C(17)$ & $-0.4(4)$ \\
\hline$C(15)-C(16)-C(17)-C(18)$ & $-0.2(4)$ \\
\hline$C(16)-C(17)-C(18)-C(13)$ & $1.3(4)$ \\
\hline $\mathrm{C}(14)-\mathrm{C}(13)-\mathrm{C}(18)-\mathrm{C}(17)$ & $-2.0(3)$ \\
\hline$P(1)-C(13)-C(18)-C(17)$ & $-174.94(18)$ \\
\hline$C(24)-C(19)-C(20)-C(21)$ & $-2.8(4)$ \\
\hline$P(2)-C(19)-C(20)-C(21)$ & $171.4(2)$ \\
\hline$C(19)-C(20)-C(21)-C(22)$ & $-0.3(4)$ \\
\hline$C(20)-C(21)-C(22)-C(23)$ & $2.8(4)$ \\
\hline $\mathrm{C}(21)-\mathrm{C}(22)-\mathrm{C}(23)-\mathrm{C}(24)$ & $-2.1(4)$ \\
\hline$C(20)-C(19)-C(24)-C(23)$ & $3.5(4)$ \\
\hline$P(2)-C(19)-C(24)-C(23)$ & $-170.9(2)$ \\
\hline $\mathrm{C}(22)-\mathrm{C}(23)-\mathrm{C}(24)-\mathrm{C}(19)$ & $-1.1(4)$ \\
\hline$C(30)-C(25)-C(26)-C(27)$ & $-0.1(4)$ \\
\hline$P(2)-C(25)-C(26)-C(27)$ & $-175.7(2)$ \\
\hline$C(25)-C(26)-C(27)-C(28)$ & $1.1(4)$ \\
\hline$C(26)-C(27)-C(28)-C(29)$ & $-1.0(5)$ \\
\hline$C(27)-C(28)-C(29)-C(30)$ & $-0.2(5)$ \\
\hline$C(28)-C(29)-C(30)-C(25)$ & $1.2(4)$ \\
\hline$C(26)-C(25)-C(30)-C(29)$ & $-1.0(4)$ \\
\hline$P(2)-C(25)-C(30)-C(29)$ & $174.7(2)$ \\
\hline$C(36)-C(31)-C(32)-C(33)$ & $1.2(4)$ \\
\hline$P(2)-C(31)-C(32)-C(33)$ & $-177.05(19)$ \\
\hline$C(31)-C(32)-C(33)-C(34)$ & $1.1(4)$ \\
\hline$C(32)-C(33)-C(34)-C(35)$ & $-2.1(4)$ \\
\hline$C(33)-C(34)-C(35)-C(36)$ & $0.6(4)$ \\
\hline$C(34)-C(35)-C(36)-C(31)$ & $1.8(4)$ \\
\hline$C(32)-C(31)-C(36)-C(35)$ & $-2.6(4)$ \\
\hline$P(2)-C(31)-C(36)-C(35)$ & $175.50(19)$ \\
\hline $\mathrm{N}(1)-\mathrm{C}(37)-\mathrm{C}(38)-\mathrm{C}(39)$ & $-2.1(3)$ \\
\hline$C(46)-C(37)-C(38)-C(39)$ & $167.4(2)$ \\
\hline$C(37)-C(38)-C(39)-N(2)$ & $0.6(3)$ \\
\hline$N(4)-C(40)-C(41)-C(42)$ & $1.2(3)$ \\
\hline$C(52)-C(40)-C(41)-C(42)$ & $-176.5(2)$ \\
\hline
\end{tabular}




$$
\begin{aligned}
& C(40)-C(41)-C(42)-\mathrm{N}(3) \\
& \mathrm{N}(6)-\mathrm{C}(43)-\mathrm{C}(44)-\mathrm{C}(45) \\
& \mathrm{C}(58)-\mathrm{C}(43)-\mathrm{C}(44)-\mathrm{C}(45) \\
& \mathrm{C}(43)-\mathrm{C}(44)-\mathrm{C}(45)-\mathrm{N}(5) \\
& \mathrm{N}(1)-\mathrm{C}(37)-\mathrm{C}(46)-\mathrm{C}(47) \\
& \mathrm{C}(38)-\mathrm{C}(37)-\mathrm{C}(46)-\mathrm{C}(47) \\
& \mathrm{N}(1)-\mathrm{C}(37)-\mathrm{C}(46)-\mathrm{C}(51) \\
& \mathrm{C}(38)-\mathrm{C}(37)-\mathrm{C}(46)-\mathrm{C}(51) \\
& \mathrm{C}(51)-\mathrm{C}(46)-\mathrm{C}(47)-\mathrm{C}(48) \\
& \mathrm{C}(37)-\mathrm{C}(46)-\mathrm{C}(47)-\mathrm{C}(48) \\
& \mathrm{C}(46)-\mathrm{C}(47)-\mathrm{C}(48)-\mathrm{C}(49) \\
& \mathrm{C}(47)-\mathrm{C}(48)-\mathrm{C}(49)-\mathrm{C}(50) \\
& \mathrm{C}(48)-\mathrm{C}(49)-\mathrm{C}(50)-\mathrm{C}(51) \\
& \mathrm{C}(49)-\mathrm{C}(50)-\mathrm{C}(51)-\mathrm{C}(46) \\
& \mathrm{C}(47)-\mathrm{C}(46)-\mathrm{C}(51)-\mathrm{C}(50) \\
& \mathrm{C}(37)-\mathrm{C}(46)-\mathrm{C}(51)-\mathrm{C}(50) \\
& \mathrm{N}(4)-\mathrm{C}(40)-\mathrm{C}(52)-\mathrm{C}(53) \\
& \mathrm{C}(41)-\mathrm{C}(40)-\mathrm{C}(52)-\mathrm{C}(53) \\
& \mathrm{N}(4)-\mathrm{C}(40)-\mathrm{C}(52)-\mathrm{C}(57) \\
& \mathrm{C}(41)-\mathrm{C}(40)-\mathrm{C}(52)-\mathrm{C}(57) \\
& \mathrm{C}(57)-\mathrm{C}(52)-\mathrm{C}(53)-\mathrm{C}(54) \\
& \mathrm{C}(40)-\mathrm{C}(52)-\mathrm{C}(53)-\mathrm{C}(54) \\
& \mathrm{C}(52)-\mathrm{C}(53)-\mathrm{C}(54)-\mathrm{C}(55) \\
& \mathrm{C}(53)-\mathrm{C}(54)-\mathrm{C}(55)-\mathrm{C}(56) \\
& \mathrm{C}(54)-\mathrm{C}(55)-\mathrm{C}(56)-\mathrm{C}(57) \\
& \mathrm{C}(55)-\mathrm{C}(56)-\mathrm{C}(57)-\mathrm{C}(52) \\
& \mathrm{C}(53)-\mathrm{C}(52)-\mathrm{C}(57)-\mathrm{C}(56) \\
& \mathrm{C}(40)-\mathrm{C}(52)-\mathrm{C}(57)-\mathrm{C}(56) \\
& \mathrm{N}(6)-\mathrm{C}(43)-\mathrm{C}(58)-\mathrm{C}(63) \\
& \mathrm{C}(44)-\mathrm{C}(43)-\mathrm{C}(58)-\mathrm{C}(63) \\
& \mathrm{N}(6)-\mathrm{C}(43)-\mathrm{C}(58)-\mathrm{C}(59) \\
& \mathrm{C}(44)-\mathrm{C}(43)-\mathrm{C}(58)-\mathrm{C}(59) \\
& \mathrm{C}(63)-\mathrm{C}(58)-\mathrm{C}(59)-\mathrm{C}(60) \\
& \mathrm{C}(43)-\mathrm{C}(58)-\mathrm{C}(59)-\mathrm{C}(60) \\
& \mathrm{C}(58)-\mathrm{C}(59)-\mathrm{C}(60)-\mathrm{C}(61) \\
& \mathrm{C}(59)-\mathrm{C}(60)-\mathrm{C}(61)-\mathrm{C}(62) \\
& \mathrm{C}(60)-\mathrm{C}(61)-\mathrm{C}(62)-\mathrm{C}(63) \\
& \mathrm{C}(59)-\mathrm{C}(58)-\mathrm{C}(63)-\mathrm{C}(62) \\
& \mathrm{C}(43)-\mathrm{C}(58)-\mathrm{C}(63)-\mathrm{C}(62) \\
& \mathrm{C}(61)-\mathrm{C}(62)-\mathrm{C}(63)-\mathrm{C}(58) \\
& \mathrm{C}(64)-\mathrm{C}(65)-\mathrm{C}(66)-\mathrm{C}(67) \\
& \mathrm{C}(65)-\mathrm{C}(66)-\mathrm{C}(67)-\mathrm{C}(68) \\
& \mathrm{C}(38)-\mathrm{C}(39)-\mathrm{N}(2)-\mathrm{N}(1) \\
& \mathrm{C}(38)-\mathrm{C}(39)-\mathrm{N}(2)-\mathrm{B}(1) \\
& \mathrm{N}(5)-\mathrm{B}(1)-\mathrm{N}(2)-\mathrm{C}(39) \\
& \mathrm{N}(3)-\mathrm{B}(1)-\mathrm{N}(2)-\mathrm{C}(39) \\
& \mathrm{N}(5)-\mathrm{B}(1)-\mathrm{N}(2)-\mathrm{N}(1) \\
& \mathrm{C}(1)-\mathrm{N}(2)-\mathrm{N}(1)-\mathrm{C}(37) \\
& \mathrm{C}(39)-\mathrm{N}(2)-\mathrm{N}(1)-\mathrm{Rh}(1) \\
& \mathrm{B}(1)-\mathrm{N}(2)-\mathrm{N}(1)-\mathrm{Rh}(1) \\
& \mathrm{C}(44)-\mathrm{C}(45)-\mathrm{N}(5)-\mathrm{N}(6) \\
& \mathrm{C}(38)-\mathrm{C}(37)-\mathrm{N}(1)-\mathrm{N}) \mathrm{N}(2) \\
& \mathrm{C}(46)-\mathrm{C}(37)-\mathrm{N}(1)-\mathrm{N}(2) \\
& \mathrm{C}(46)-\mathrm{C}(37)-\mathrm{N}(1)-\mathrm{Rh}(1) \\
&
\end{aligned}
$$

$-1.1(3)$

$0.0(3)$

$-177.7(2)$

$0.1(3)$

$-136.0(2)$

$55.6(4)$

$49.0(3)$

$-119.5(3)$

$0.0(4)$

$-175.0(2)$

$-0.5(4)$

$0.7(5)$

$-0.3(4)$

$-0.3(4)$

$0.4(4)$

$175.7(2)$

$-23.1(4)$

$154.3(3)$

$159.5(2)$

$-23.1(4)$

$-0.2(4)$

$-177.6(2)$

$0.7(4)$

$-0.6(4)$

$0.1(5)$

$0.4(4)$

$-0.4(4)$

$177.1(2)$

$-15.1(3)$

$162.4(3)$

$165.9(2)$

$-16.6(4)$

$-1.0(4)$

$178.1(2)$

$0.9(4)$

$0.0(4)$

$-0.7(4)$

$0.2(4)$

$-178.8(2)$

$0.6(4)$

$-178.5(7)$

$-159.5(7)$

$1.0(3)$

$-167.2(2)$

$-2.5(4)$

$116.7(3)$

$-169.96(19)$

$-50.8(3)$

$2.7(3)$

$-167.8(2)$

-165.57 (19)

$24.0(4)$

$-2.3(3)$

$168.0(2)$

$169.30(16)$

$-20.4(2)$

$-0.1(3)$ 


\begin{tabular}{|c|c|}
\hline $\mathrm{C}(44)-\mathrm{C}(45)-\mathrm{N}(5)-\mathrm{B}(1)$ & $-173.7(2)$ \\
\hline $\mathrm{N}(3)-\mathrm{B}(1)-\mathrm{N}(5)-\mathrm{C}(45)$ & $105.7(3)$ \\
\hline $\mathrm{N}(2)-\mathrm{B}(1)-\mathrm{N}(5)-\mathrm{C}(45)$ & $-138.2(2)$ \\
\hline $\mathrm{N}(3)-\mathrm{B}(1)-\mathrm{N}(5)-\mathrm{N}(6)$ & $-67.0(3)$ \\
\hline $\mathrm{N}(2)-\mathrm{B}(1)-\mathrm{N}(5)-\mathrm{N}(6)$ & $49.1(3)$ \\
\hline $\mathrm{C}(44)-\mathrm{C}(43)-\mathrm{N}(6)-\mathrm{N}(5)$ & $-0.1(3)$ \\
\hline $\mathrm{C}(58)-\mathrm{C}(43)-\mathrm{N}(6)-\mathrm{N}(5)$ & $177.8(2)$ \\
\hline$C(45)-N(5)-N(6)-C(43)$ & $0.1(3)$ \\
\hline $\mathrm{B}(1)-\mathrm{N}(5)-\mathrm{N}(6)-\mathrm{C}(43)$ & $173.7(2)$ \\
\hline $\mathrm{C}(41)-\mathrm{C}(42)-\mathrm{N}(3)-\mathrm{N}(4)$ & $0.6(3)$ \\
\hline $\mathrm{C}(41)-\mathrm{C}(42)-\mathrm{N}(3)-\mathrm{B}(1)$ & $167.7(2)$ \\
\hline $\mathrm{N}(5)-\mathrm{B}(1)-\mathrm{N}(3)-\mathrm{C}(42)$ & $16.5(4)$ \\
\hline $\mathrm{N}(2)-\mathrm{B}(1)-\mathrm{N}(3)-\mathrm{C}(42)$ & $-103.7(3)$ \\
\hline $\mathrm{N}(5)-\mathrm{B}(1)-\mathrm{N}(3)-\mathrm{N}(4)$ & $-177.46(18)$ \\
\hline $\mathrm{N}(2)-\mathrm{B}(1)-\mathrm{N}(3)-\mathrm{N}(4)$ & $62.3(2)$ \\
\hline $\mathrm{C}(41)-\mathrm{C}(40)-\mathrm{N}(4)-\mathrm{N}(3)$ & $-0.8(3)$ \\
\hline$C(52)-C(40)-N(4)-N(3)$ & $176.9(2)$ \\
\hline $\mathrm{C}(41)-\mathrm{C}(40)-\mathrm{N}(4)-\mathrm{Rh}(1)$ & $-170.32(18)$ \\
\hline $\mathrm{C}(52)-\mathrm{C}(40)-\mathrm{N}(4)-\mathrm{Rh}(1)$ & $7.5(4)$ \\
\hline $\mathrm{C}(42)-\mathrm{N}(3)-\mathrm{N}(4)-\mathrm{C}(40)$ & $0.2(2)$ \\
\hline $\mathrm{B}(1)-\mathrm{N}(3)-\mathrm{N}(4)-\mathrm{C}(40)$ & $-168.6(2)$ \\
\hline $\mathrm{C}(42)-\mathrm{N}(3)-\mathrm{N}(4)-\mathrm{Rh}(1)$ & $172.55(16)$ \\
\hline $\mathrm{B}(1)-\mathrm{N}(3)-\mathrm{N}(4)-\mathrm{Rh}(1)$ & $3.8(2)$ \\
\hline$C(14)-C(13)-P(1)-C(7)$ & $126.22(18)$ \\
\hline$C(18)-C(13)-P(1)-C(7)$ & $-60.8(2)$ \\
\hline$C(14)-C(13)-P(1)-C(1)$ & $-130.14(18)$ \\
\hline$C(18)-C(13)-P(1)-C(1)$ & $42.8(2)$ \\
\hline $\mathrm{C}(14)-\mathrm{C}(13)-\mathrm{P}(1)-\mathrm{Rh}(1)$ & $-6.8(2)$ \\
\hline $\mathrm{C}(18)-\mathrm{C}(13)-\mathrm{P}(1)-\mathrm{Rh}(1)$ & $166.13(17)$ \\
\hline$C(8)-C(7)-P(1)-C(13)$ & $-164.83(18)$ \\
\hline$C(12)-C(7)-P(1)-C(13)$ & $15.2(2)$ \\
\hline$C(8)-C(7)-P(1)-C(1)$ & $93.6(2)$ \\
\hline$C(12)-C(7)-P(1)-C(1)$ & $-86.4(2)$ \\
\hline $\mathrm{C}(8)-\mathrm{C}(7)-\mathrm{P}(1)-\mathrm{Rh}(1)$ & $-35.1(2)$ \\
\hline $\mathrm{C}(12)-\mathrm{C}(7)-\mathrm{P}(1)-\mathrm{Rh}(1)$ & $144.91(19)$ \\
\hline$C(6)-C(1)-P(1)-C(13)$ & $-129.3(2)$ \\
\hline$C(2)-C(1)-P(1)-C(13)$ & $48.1(2)$ \\
\hline$C(6)-C(1)-P(1)-C(7)$ & $-20.2(2)$ \\
\hline$C(2)-C(1)-P(1)-C(7)$ & $157.17(19)$ \\
\hline $\mathrm{C}(6)-\mathrm{C}(1)-\mathrm{P}(1)-\mathrm{Rh}(1)$ & $110.06(19)$ \\
\hline $\mathrm{C}(2)-\mathrm{C}(1)-\mathrm{P}(1)-\mathrm{Rh}(1)$ & $-72.61(19)$ \\
\hline$C(26)-C(25)-P(2)-C(31)$ & $-9.0(2)$ \\
\hline$C(30)-C(25)-P(2)-C(31)$ & $175.45(19)$ \\
\hline$C(26)-C(25)-P(2)-C(19)$ & $95.9(2)$ \\
\hline$C(30)-C(25)-P(2)-C(19)$ & $-79.6(2)$ \\
\hline $\mathrm{C}(26)-\mathrm{C}(25)-\mathrm{P}(2)-\mathrm{Rh}(1)$ & $-135.10(19)$ \\
\hline $\mathrm{C}(30)-\mathrm{C}(25)-\mathrm{P}(2)-\mathrm{Rh}(1)$ & $49.4(2)$ \\
\hline$C(32)-C(31)-P(2)-C(25)$ & $-101.60(19)$ \\
\hline$C(36)-C(31)-P(2)-C(25)$ & $80.3(2)$ \\
\hline$C(32)-C(31)-P(2)-C(19)$ & $155.22(19)$ \\
\hline$C(36)-C(31)-P(2)-C(19)$ & $-22.9(2)$ \\
\hline$C(32)-C(31)-P(2)-R h(1)$ & $22.9(2)$ \\
\hline $\mathrm{C}(36)-\mathrm{C}(31)-\mathrm{P}(2)-\mathrm{Rh}(1)$ & $-155.19(18)$ \\
\hline$C(20)-C(19)-P(2)-C(25)$ & $29.8(2)$ \\
\hline$C(24)-C(19)-P(2)-C(25)$ & $-156.00(19)$ \\
\hline$C(20)-C(19)-P(2)-C(31)$ & $136.6(2)$ \\
\hline$C(24)-C(19)-P(2)-C(31)$ & $-49.2(2)$ \\
\hline
\end{tabular}




\begin{tabular}{|c|c|}
\hline$C(20)-C(19)-P(2)-R h(1)$ & $-94.2(2)$ \\
\hline$C(24)-C(19)-P(2)-R h(1)$ & $80.0(2)$ \\
\hline $\mathrm{C}(40)-\mathrm{N}(4)-\mathrm{Rh}(1)-\mathrm{N}(1)$ & $112.6(2)$ \\
\hline $\mathrm{N}(3)-\mathrm{N}(4)-\mathrm{Rh}(1)-\mathrm{N}(1)$ & $-56.39(14)$ \\
\hline $\mathrm{C}(40)-\mathrm{N}(4)-\mathrm{Rh}(1)-\mathrm{P}(1)$ & $-76.3(2)$ \\
\hline $\mathrm{N}(3)-\mathrm{N}(4)-\mathrm{Rh}(1)-\mathrm{P}(1)$ & $114.76(14)$ \\
\hline $\mathrm{C}(40)-\mathrm{N}(4)-\mathrm{Rh}(1)-\mathrm{P}(2)$ & $123.1(3)$ \\
\hline $\mathrm{N}(3)-\mathrm{N}(4)-\mathrm{Rh}(1)-\mathrm{P}(2)$ & $-45.9(4)$ \\
\hline $\mathrm{C}(37)-\mathrm{N}(1)-\mathrm{Rh}(1)-\mathrm{N}(4)$ & $-125.8(2)$ \\
\hline $\mathrm{N}(2)-\mathrm{N}(1)-\mathrm{Rh}(1)-\mathrm{N}(4)$ & $66.39(15)$ \\
\hline $\mathrm{C}(37)-\mathrm{N}(1)-\mathrm{Rh}(1)-\mathrm{P}(1)$ & $-170.11(18)$ \\
\hline $\mathrm{N}(2)-\mathrm{N}(1)-\mathrm{Rh}(1)-\mathrm{P}(1)$ & $22.1(4)$ \\
\hline $\mathrm{C}(37)-\mathrm{N}(1)-\mathrm{Rh}(1)-\mathrm{P}(2)$ & $55.7(2)$ \\
\hline $\mathrm{N}(2)-\mathrm{N}(1)-\mathrm{Rh}(1)-\mathrm{P}(2)$ & $-112.04(14)$ \\
\hline $\mathrm{C}(13)-\mathrm{P}(1)-\mathrm{Rh}(1)-\mathrm{N}(4)$ & $-101.43(9)$ \\
\hline $\mathrm{C}(7)-\mathrm{P}(1)-\mathrm{Rh}(1)-\mathrm{N}(4)$ & $131.56(10)$ \\
\hline $\mathrm{C}(1)-\mathrm{P}(1)-\mathrm{Rh}(1)-\mathrm{N}(4)$ & $10.71(10)$ \\
\hline $\mathrm{C}(13)-\mathrm{P}(1)-\mathrm{Rh}(1)-\mathrm{N}(1)$ & $-58.2(3)$ \\
\hline $\mathrm{C}(7)-\mathrm{P}(1)-\mathrm{Rh}(1)-\mathrm{N}(1)$ & $174.8(3)$ \\
\hline $\mathrm{C}(1)-\mathrm{P}(1)-\mathrm{Rh}(1)-\mathrm{N}(1)$ & $54.0(3)$ \\
\hline$C(13)-P(1)-R h(1)-P(2)$ & $75.70(8)$ \\
\hline $\mathrm{C}(7)-\mathrm{P}(1)-\mathrm{Rh}(1)-\mathrm{P}(2)$ & $-51.31(9)$ \\
\hline $\mathrm{C}(1)-\mathrm{P}(1)-\mathrm{Rh}(1)-\mathrm{P}(2)$ & $-172.16(9)$ \\
\hline $\mathrm{C}(25)-\mathrm{P}(2)-\mathrm{Rh}(1)-\mathrm{N}(4)$ & $5.9(4)$ \\
\hline $\mathrm{C}(31)-\mathrm{P}(2)-\mathrm{Rh}(1)-\mathrm{N}(4)$ & $-113.9(4)$ \\
\hline $\mathrm{C}(19)-\mathrm{P}(2)-\mathrm{Rh}(1)-\mathrm{N}(4)$ & $123.2(4)$ \\
\hline $\mathrm{C}(25)-\mathrm{P}(2)-\mathrm{Rh}(1)-\mathrm{N}(1)$ & $16.28(10)$ \\
\hline $\mathrm{C}(31)-\mathrm{P}(2)-\mathrm{Rh}(1)-\mathrm{N}(1)$ & $-103.51(10)$ \\
\hline $\mathrm{C}(19)-\mathrm{P}(2)-\mathrm{Rh}(1)-\mathrm{N}(1)$ & $133.57(11)$ \\
\hline$C(25)-P(2)-R h(1)-P(1)$ & $-154.57(9)$ \\
\hline $\mathrm{C}(31)-\mathrm{P}(2)-\mathrm{Rh}(1)-\mathrm{P}(1)$ & $85.64(8)$ \\
\hline$C(19)-P(2)-R h(1)-P(1)$ & $-37.28(10)$ \\
\hline$C(67 B)-C(66 B)-C(65 B)-C(64 B)$ & $56.9(18)$ \\
\hline
\end{tabular}


Figure S7. Crystal Structure of Complex 5 - Configuration represented in paper.

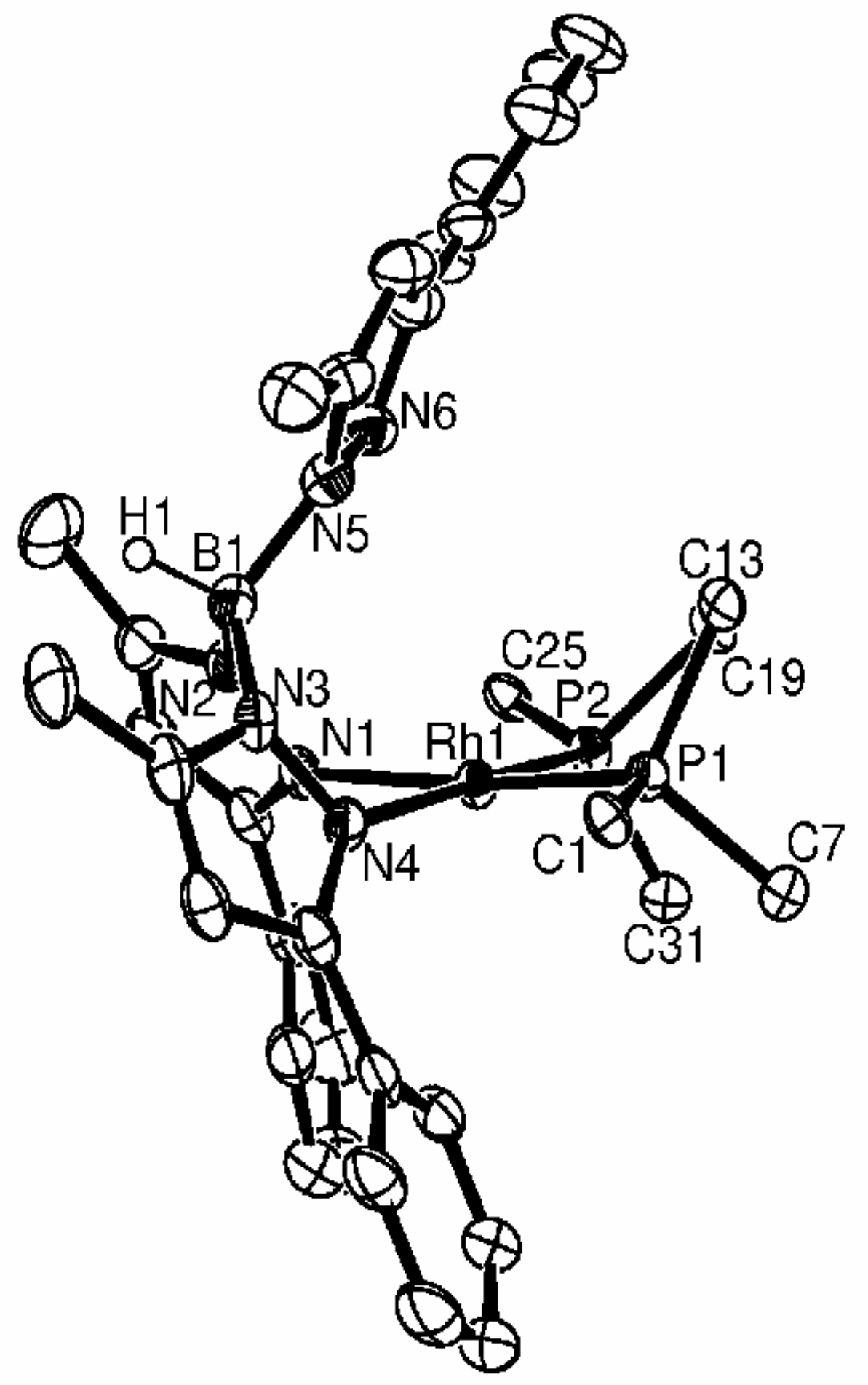


Figure S8. Crystal Structure of Complex 5 - Including all atoms.

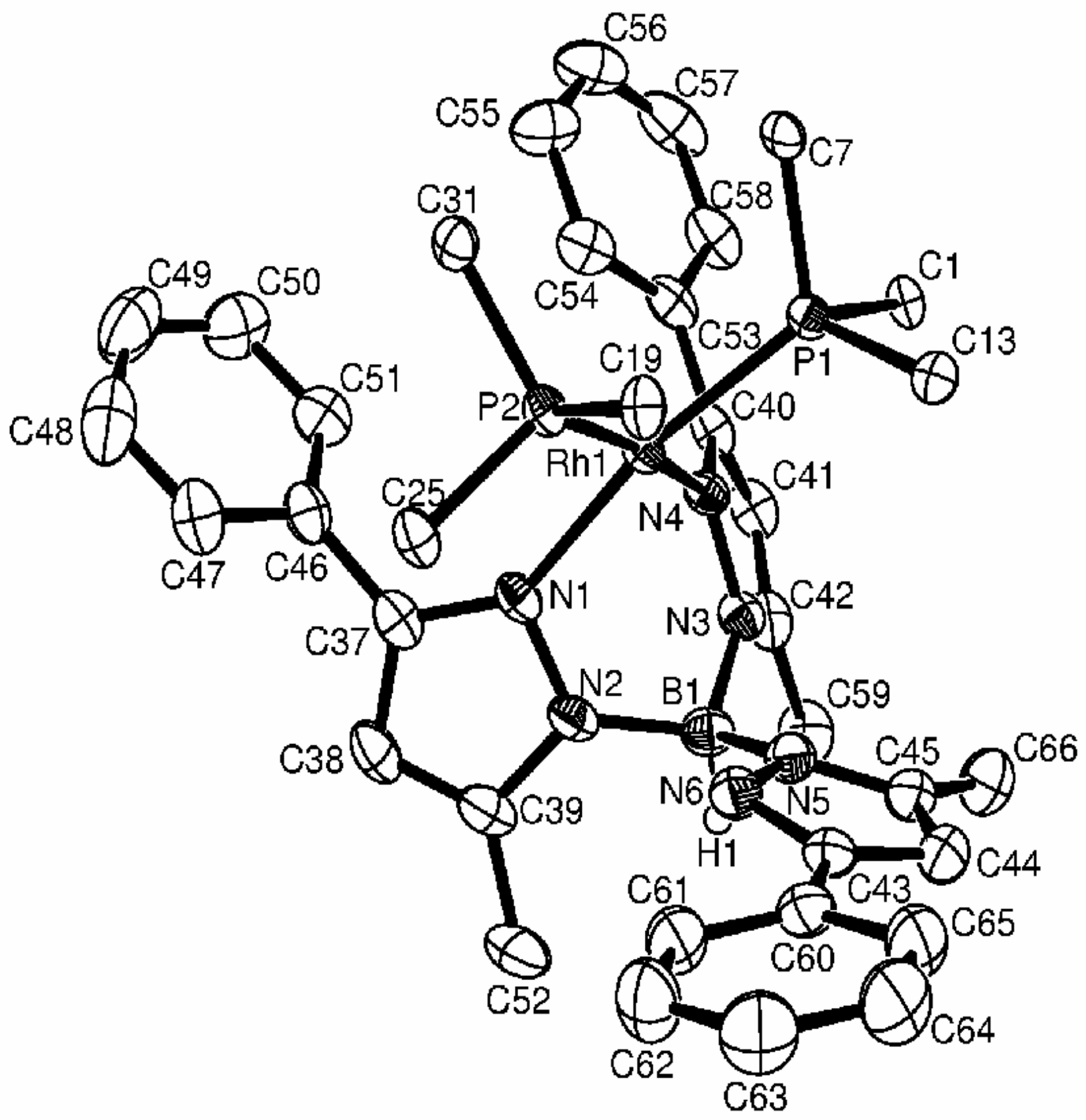




\section{Experimental}

\section{Data Collection}

An irregular red crystal of $\mathrm{C}_{66} \mathrm{H}_{58} \mathrm{BN}_{6} \mathrm{P}_{2}$ Rh. $\mathrm{C}_{6} \mathrm{H}_{14}$ having approximate dimensions of $0.05 \times 0.12 \times$ $0.25 \mathrm{~mm}$ was mounted on a glass fiber. All measurements were made on a Bruker X8 APEX II diffractometer with graphite monochromated Mo-Ka radiation.

The data were collected at a temperature of $-100.0 \pm 0.1^{\circ} \mathrm{C}$ to a maximum $2 \theta$ value of $50.1^{\circ}$. Data were collected in a series of $\phi$ and $\omega$ scans in $0.50^{\circ}$ oscillations with 30.0 second exposures. The crystal-todetector distance was $36.00 \mathrm{~mm}$.

\section{Data Reduction}

Of the 49830 reflections that were collected, 11499 were unique $\left(R_{i n t}=0.052\right)$; equivalent reflections were merged. Data were collected and integrated using the Bruker SAINT ${ }^{1}$ software package. The linear absorption coefficient, $\mu$, for Mo-K $\alpha$ radiation is $3.57 \mathrm{~cm}^{-1}$. Data were corrected for absorption effects using the multi-scan technique $\left(\operatorname{SADABS}^{2}\right.$ ), with minimum and maximum transmission coefficients of 0.842 and 0.982, respectively. The data were corrected for Lorentz and polarization effects.

\section{$\underline{\text { Structure Solution and Refinement }}$}

The structure was solved by direct methods ${ }^{3}$. The material crystallizes with disordered hexanes in the lattice. This disordered solvent molecule could not be modeled reasonably, therefore the PLATON/SQUEEZE ${ }^{11}$ program was used to correct the data for any unresolved residual electron density in the lattice. The formula and any subsequent values calculated from it reflect the presence of one molecule of hexane in the asymmetric unit. All non-hydrogen atoms were refined anisotropically, while all hydrogen atoms except $\mathrm{H} 1$ were placed in calculated positions but were not refined. $\mathrm{H} 1$ was located in a difference map and refined isotropically. The final cycle of full-matrix least-squares refinement ${ }^{4}$ on $\mathrm{F}^{2}$ was based on 11499 reflections and 692 variable parameters and converged (largest parameter shift was 0.00 times its esd) with unweighted and weighted agreement factors of:

$$
\mathrm{R} 1=\Sigma|| \mathrm{Fo}|-| \mathrm{Fc} \| / \Sigma|\mathrm{Fo}|=0.058
$$




$$
w R 2=\left[\Sigma\left(w\left(F o^{2}-F_{c}^{2}\right)^{2}\right) / \Sigma w\left(F o^{2}\right)^{2}\right]^{1 / 2}=0.090
$$

The standard deviation of an observation of unit weight ${ }^{5}$ was 0.99 . The weighting scheme was based on counting statistics. The maximum and minimum peaks on the final difference Fourier map corresponded to 0.46 and $-0.31 \mathrm{e}^{-} / \AA^{3}$, respectively.

Neutral atom scattering factors were taken from Cromer and Waber 6 . Anomalous dispersion effects were included in $\mathrm{Fcalc}^{7}$; the values for $\Delta \mathrm{f}^{\prime}$ and $\Delta \mathrm{f}^{\prime \prime}$ were those of Creagh and McAuley ${ }^{8}$. The values for the mass attenuation coefficients are those of Creagh and Hubbell ${ }^{9}$. All refinements were performed using the SHELXTL 10 crystallographic software package of Bruker-AXS.

\section{References}

(1) SAINT. Version 7.03A. Bruker AXS Inc., Madison, Wisconsin, USA. (1997-2003).

(2) SADABS. Bruker Nonius area detector scaling and absorption correction - V2.10, Bruker AXS Inc., Madison, Wisconsin, USA (2003).

(3) SIR97 - Altomare A., Burla M.C., Camalli M., Cascarano G.L., Giacovazzo C. , Guagliardi A., Moliterni A.G.G., Polidori G.,Spagna R. (1999) J. Appl. Cryst. 32, 115-119.

(4) Least Squares function minimized:

$$
\Sigma w\left(\mathrm{~F}_{\mathrm{O}}{ }^{2}-\mathrm{F}_{\mathrm{C}}{ }^{2}\right)^{2}
$$

(5) Standard deviation of an observation of unit weight:

$$
\begin{aligned}
& {\left[\Sigma w\left(\mathrm{~F}_{\mathrm{O}}^{2}-\mathrm{F}_{\mathrm{C}}^{2}\right)^{2} /\left(\mathrm{N}_{\mathrm{O}}-\mathrm{N}_{\mathrm{V}}\right)\right]^{1 / 2}} \\
& \text { where: } \mathrm{N}_{\mathrm{O}}=\text { number of observations } \\
& \qquad \mathrm{N}_{\mathrm{V}}=\text { number of variables }
\end{aligned}
$$

(6) Cromer, D. T. \& Waber, J. T.; "International Tables for X-ray Crystallography", Vol. IV, The Kynoch Press, Birmingham, England, Table 2.2 A (1974).

(7) Ibers, J. A. \& Hamilton, W. C.; Acta Crystallogr., 17, 781 (1964).

(8) Creagh, D. C. \& McAuley, W.J .; "International Tables for Crystallography", Vol C, (A.J.C. Wilson, ed.), Kluwer Academic Publishers, Boston, Table 4.2.6.8, pages 219-222 (1992).

(9) Creagh, D. C. \& Hubbell, J.H..; "International Tables for Crystallography", Vol C, (A.J.C. Wilson, ed.), Kluwer Academic Publishers, Boston, Table 4.2.4.3, pages 200-206 (1992).

(10) SHELXTL Version 5.1. Bruker AXS Inc., Madision, Wisconsin, USA. (1997).

(11) SQUEEZE - Sluis, P. v.d.; Spek, A. L. Acta Crystallogr., Sect A 1990, 46, 194-201. 
Table S19. Crystal Data and Structure Analysis Details for Complex 5.

A. Crystal Data

\author{
Empirical Formula \\ Formula Weight \\ Crystal Color, Habit \\ Crystal Dimensions \\ Crystal System \\ Lattice Type \\ Lattice Parameters
}

$\mathrm{C}_{72} \mathrm{H}_{72} \mathrm{BN}_{6} \mathrm{P}_{2} \mathrm{Rh}$

1197.02

red, irregular

$0.05 \times 0.12 \times 0.25 \mathrm{~mm}$

monoclinic

primitive

$$
\begin{aligned}
& a=14.9000(5) \AA \\
& b=14.8303(5) \AA \\
& c=29.460(1) \AA \\
& \alpha=90.00 \\
& \beta=92.260(2) \circ \\
& \gamma=90.00 \\
& V=6504.8(4) \AA^{3}
\end{aligned}
$$

Space Group

$P 21 / n(\# 14)$

$Z$ value

4

$\mathrm{D}_{\text {calc }}$

$1.222 \mathrm{~g} / \mathrm{cm}^{3}$

F000

2504.00

$\mu(\operatorname{MoK} \alpha)$

$3.57 \mathrm{~cm}^{-1}$ 
B. Intensity Measurements

Diffractometer

Radiation

Data Images

Detector Position

$2 \theta \max$

No. of Reflections Measured

Corrections
Bruker X8 APEX II

$\operatorname{MoK} \alpha(\lambda=0.71073 \AA)$

graphite monochromated

1138 exposures@ 30.0 seconds

$36.00 \mathrm{~mm}$

50.10

Total: 49830

Unique: $11499\left(R_{\text {int }}=0.052\right)$

Absorption $\left(T_{\min }=0.842, T_{\max }=0.982\right)$ Lorentz-polarization 
C. Structure Solution and Refinement

\begin{tabular}{|c|c|}
\hline Structure Solution & Direct Methods (SIR97) \\
\hline Refinement & Full-matrix least-squares on $\mathrm{F}^{2}$ \\
\hline Function Minimized & $\Sigma w\left(F o^{2}-F^{2}\right)^{2}$ \\
\hline Least Squares Weights & $w=1 /\left(\sigma^{2}\left(F^{2}\right)+(0.0474 P)^{2}+0.0000 P\right)$ \\
\hline Anomalous Dispersion & All non-hydrogen atoms \\
\hline No. Observations $(I>0.00 \sigma(I))$ & 11499 \\
\hline No. Variables & 692 \\
\hline Reflection/Parameter Ratio & 16.62 \\
\hline Residuals (refined on $\mathrm{F}^{2}$, all data): R1; wR2 & $0.058 ; 0.090$ \\
\hline Goodness of Fit Indicator & 0.99 \\
\hline No. Observations $(I>2.00 \sigma(I))$ & 8482 \\
\hline Residuals (refined on F): R1; wR2 & $0.037 ; 0.084$ \\
\hline Max Shift/Error in Final Cycle & 0.00 \\
\hline Maximum peak in Final Diff. Map & $0.46 \mathrm{e}^{-/} / \AA^{3}$ \\
\hline Minimum peak in Final Diff. Map & $-0.31 e^{-} / \AA^{3}$ \\
\hline
\end{tabular}


Table s20. Atomic coordinates $\left(\mathrm{x}^{\left.10^{\wedge} 4\right)}\right.$ ) and equivalent isotropic displacement parameters $\left(A^{\wedge} 2 \times 10^{\wedge} 3\right)$ for complex 5.

$\mathrm{U}(e q)$ is defined as one third of the trace of the orthogonalized Uij tensor.

\begin{tabular}{|c|c|c|c|c|}
\hline & $x$ & y & z & $\mathrm{U}(\mathrm{eq})$ \\
\hline$C(1)$ & $5415(2)$ & $2035(2)$ & $2536(1)$ & $26(1)$ \\
\hline$C(2)$ & $6219(2)$ & 1797 (2) & $2757(1)$ & $33(1)$ \\
\hline C (3) & $6762(2)$ & $1143(2)$ & $2577(1)$ & $43(1)$ \\
\hline$C(4)$ & $6513(2)$ & $711(2)$ & 2177 (1) & $47(1)$ \\
\hline$C(5)$ & $5716(2)$ & $941(2)$ & $1959(1)$ & $44(1)$ \\
\hline$C(6)$ & $5164(2)$ & $1596(2)$ & $2131(1)$ & $35(1)$ \\
\hline C (7) & $3900(2)$ & $3147(2)$ & $2327(1)$ & $24(1)$ \\
\hline$C(8)$ & $4142(2)$ & $3886(2)$ & $2067(1)$ & $32(1)$ \\
\hline$C(9)$ & $3622(2)$ & $4140(2)$ & $1694(1)$ & $41(1)$ \\
\hline$C(10)$ & $2841(2)$ & $3677(2)$ & $1577(1)$ & $42(1)$ \\
\hline$C(11)$ & $2604(2)$ & $2946(2)$ & $1827(1)$ & $37(1)$ \\
\hline C (12) & $3132(2)$ & $2671(2)$ & $2197(1)$ & $31(1)$ \\
\hline C (13) & $3998(2)$ & $2042(2)$ & $3134(1)$ & $24(1)$ \\
\hline$C(14)$ & $4384(2)$ & $1252(2)$ & $3311(1)$ & $30(1)$ \\
\hline$C(15)$ & 3907 (2) & $697(2)$ & $3589(1)$ & $40(1)$ \\
\hline$C(16)$ & $3040(2)$ & $909(2)$ & $3696(1)$ & $40(1)$ \\
\hline C (17) & $2642(2)$ & $1676(2)$ & $3517(1)$ & $37(1)$ \\
\hline$C(18)$ & $3113(2)$ & $2241(2)$ & $3239(1)$ & $29(1)$ \\
\hline C (19) & $3892(2)$ & $3620(2)$ & $4054(1)$ & $22(1)$ \\
\hline$C(20)$ & $4378(2)$ & $2833(2)$ & $4125(1)$ & $26(1)$ \\
\hline C (21) & $4164(2)$ & $2254(2)$ & $4477(1)$ & $34(1)$ \\
\hline C (22) & $3485(2)$ & $2459(2)$ & $4759(1)$ & $37(1)$ \\
\hline C (23) & $3029(2)$ & $3264(2)$ & $4707(1)$ & $36(1)$ \\
\hline C (24) & $3243(2)$ & $3851(2)$ & $4363(1)$ & $30(1)$ \\
\hline C (25) & $3031(2)$ & $4541(2)$ & $3278(1)$ & $22(1)$ \\
\hline C (26) & $2198(2)$ & $4311(2)$ & $3441(1)$ & $33(1)$ \\
\hline$C(27)$ & $1411(2)$ & $4495(2)$ & 3187 (1) & $40(1)$ \\
\hline C (28) & 1445 (2) & $4904(2)$ & $2770(1)$ & $36(1)$ \\
\hline C (29) & $2260(2)$ & $5122(2)$ & $2601(1)$ & $36(1)$ \\
\hline C (30) & $3043(2)$ & $4942(2)$ & $2853(1)$ & $30(1)$ \\
\hline$C(31)$ & $4272(2)$ & $5407(2)$ & $3905(1)$ & $22(1)$ \\
\hline C (32) & $3823(2)$ & $6201(2)$ & $3809(1)$ & $29(1)$ \\
\hline$C(33)$ & $4009(2)$ & $6967(2)$ & $4066(1)$ & $37(1)$ \\
\hline C (34) & $4647(2)$ & $6948(2)$ & $4416(1)$ & $36(1)$ \\
\hline C (35) & $5083(2)$ & $6154(2)$ & $4522(1)$ & $36(1)$ \\
\hline$C(36)$ & $4897(2)$ & $5387(2)$ & $4272(1)$ & $31(1)$ \\
\hline C (37) & $5408(2)$ & $6513(2)$ & $3063(1)$ & $30(1)$ \\
\hline C (38) & $5225(2)$ & $7428(2)$ & $3129(1)$ & $39(1)$ \\
\hline C (39) & $4668(2)$ & $7886(2)$ & $2825(1)$ & $52(1)$ \\
\hline$C(40)$ & $4270(2)$ & $7456(2)$ & $2455(1)$ & $52(1)$ \\
\hline$C(41)$ & $4450(2)$ & $6560(2)$ & $2385(1)$ & $46(1)$ \\
\hline$C(42)$ & $5011(2)$ & 6091 (2) & $2685(1)$ & $35(1)$ \\
\hline$C(43)$ & $6040(2)$ & $6041(2)$ & $3377(1)$ & $27(1)$ \\
\hline C ( 4 4) & $6746(2)$ & $6427(2)$ & $3634(1)$ & $32(1)$ \\
\hline$C(45)$ & $7218(2)$ & $5740(2)$ & $3828(1)$ & $33(1)$ \\
\hline$C(46)$ & $8066(2)$ & $5809(2)$ & $4121(1)$ & $44(1)$ \\
\hline C (47) & $6577(2)$ & $3863(2)$ & $2052(1)$ & $31(1)$ \\
\hline
\end{tabular}




\begin{tabular}{|c|c|c|c|c|}
\hline$C(48)$ & $6304(2)$ & $4740(2)$ & $1958(1)$ & $37(1)$ \\
\hline C (49) & $5982(2)$ & $4982(2)$ & $1532(1)$ & $49(1)$ \\
\hline$C(50)$ & $5919(3)$ & $4343(3)$ & $1192(1)$ & $62(1)$ \\
\hline C (51) & $6187(3)$ & $3466(3)$ & $1280(1)$ & $61(1)$ \\
\hline C (52) & $6522(2)$ & $3227(2)$ & $1705(1)$ & $43(1)$ \\
\hline C (53) & $7006(2)$ & $3635(2)$ & $2497(1)$ & $29(1)$ \\
\hline C ( 54$)$ & $7857(2)$ & $3275(2)$ & 2575 (1) & $37(1)$ \\
\hline C (55) & $8012(2)$ & $3288(2)$ & $3035(1)$ & $35(1)$ \\
\hline$C(56)$ & $8819(2)$ & $2975(3)$ & $3312(1)$ & $55(1)$ \\
\hline C (57) & $6372(2)$ & $941(2)$ & $4191(1)$ & $33(1)$ \\
\hline C ( 58$)$ & $6590(2)$ & $465(2)$ & $3807(1)$ & $40(1)$ \\
\hline C (59) & $6447(2)$ & $-449(2)$ & $3771(1)$ & $50(1)$ \\
\hline$C(60)$ & $6075(2)$ & $-915(2)$ & $4120(1)$ & $51(1)$ \\
\hline C (61) & $5852(2)$ & $-463(2)$ & $4506(1)$ & $53(1)$ \\
\hline C (62) & $6005(2)$ & $458(2)$ & $4547(1)$ & $46(1)$ \\
\hline C (63) & $6532(2)$ & $1916(2)$ & $4234(1)$ & $32(1)$ \\
\hline C (64) & $6577(2)$ & $2442(2)$ & $4632(1)$ & $37(1)$ \\
\hline C (65) & $6753(2)$ & $3306(2)$ & $4488(1)$ & $34(1)$ \\
\hline$C(66)$ & $6938(2)$ & $4126(2)$ & $4771(1)$ & $43(1)$ \\
\hline B (1) & $7236(2)$ & $3994(2)$ & $3722(1)$ & $30(1)$ \\
\hline $\mathrm{N}(1)$ & $6067(1)$ & $5140(1)$ & $3432(1)$ & $23(1)$ \\
\hline N (2) & $6808(1)$ & $4949(2)$ & $3709(1)$ & $26(1)$ \\
\hline N (3) & $7283(1)$ & $3642(2)$ & $3230(1)$ & $28(1)$ \\
\hline N ( 4 ) & $6636(1)$ & $3840(1)$ & $2895(1)$ & $25(1)$ \\
\hline $\mathrm{N}(5)$ & $6785(2)$ & $3290(2)$ & $4029(1)$ & $29(1)$ \\
\hline $\mathrm{N}(6)$ & $6659(2)$ & $2426(2)$ & $3868(1)$ & $30(1)$ \\
\hline$P(1)$ & $4671(1)$ & $2844(1)$ & $2806(1)$ & $22(1)$ \\
\hline P (2) & $4133(1)$ & $4363(1)$ & 3575 (1) & $20(1)$ \\
\hline $\mathrm{Rh}(1)$ & $5336(1)$ & 4041 (1) & $3161(1)$ & $20(1)$ \\
\hline
\end{tabular}


Table S21. Bond lengths [A] and angles [deg] for complex 5.

\begin{tabular}{|c|c|}
\hline$C(1)-C(2)$ & $1.386(4)$ \\
\hline$C(1)-C(6)$ & $1.397(4)$ \\
\hline$C(1)-P(1)$ & $1.837(3)$ \\
\hline$C(2)-C(3)$ & $1.383(4)$ \\
\hline $\mathrm{C}(2)-\mathrm{H}(2)$ & 0.9500 \\
\hline$C(3)-C(4)$ & $1.380(4)$ \\
\hline $\mathrm{C}(3)-\mathrm{H}(3)$ & 0.9500 \\
\hline$C(4)-C(5)$ & $1.371(5)$ \\
\hline $\mathrm{C}(4)-\mathrm{H}(4)$ & 0.9500 \\
\hline$C(5)-C(6)$ & $1.382(4)$ \\
\hline $\mathrm{C}(5)-\mathrm{H}(5)$ & 0.9500 \\
\hline $\mathrm{C}(6)-\mathrm{H}(6)$ & 0.9500 \\
\hline$C(7)-C(12)$ & $1.387(4)$ \\
\hline$C(7)-C(8)$ & $1.392(4)$ \\
\hline$C(7)-P(1)$ & $1.839(3)$ \\
\hline$C(8)-C(9)$ & $1.374(4)$ \\
\hline $\mathrm{C}(8)-\mathrm{H}(8)$ & 0.9500 \\
\hline$C(9)-C(10)$ & $1.384(4)$ \\
\hline $\mathrm{C}(9)-\mathrm{H}(9)$ & 0.9500 \\
\hline$C(10)-C(11)$ & $1.365(4)$ \\
\hline $\mathrm{C}(10)-\mathrm{H}(10)$ & 0.9500 \\
\hline$C(11)-C(12)$ & $1.381(4)$ \\
\hline $\mathrm{C}(11)-\mathrm{H}(11)$ & 0.9500 \\
\hline $\mathrm{C}(12)-\mathrm{H}(12)$ & 0.9500 \\
\hline$C(13)-C(14)$ & $1.397(4)$ \\
\hline$C(13)-C(18)$ & $1.397(4)$ \\
\hline$C(13)-P(1)$ & $1.852(3)$ \\
\hline$C(14)-C(15)$ & $1.377(4)$ \\
\hline $\mathrm{C}(14)-\mathrm{H}(14)$ & 0.9500 \\
\hline$C(15)-C(16)$ & $1.379(4)$ \\
\hline $\mathrm{C}(15)-\mathrm{H}(15)$ & 0.9500 \\
\hline$C(16)-C(17)$ & $1.379(4)$ \\
\hline $\mathrm{C}(16)-\mathrm{H}(16)$ & 0.9500 \\
\hline$C(17)-C(18)$ & $1.381(4)$ \\
\hline $\mathrm{C}(17)-\mathrm{H}(17)$ & 0.9500 \\
\hline $\mathrm{C}(18)-\mathrm{H}(18)$ & 0.9500 \\
\hline$C(19)-C(20)$ & $1.385(4)$ \\
\hline$C(19)-C(24)$ & $1.396(4)$ \\
\hline$C(19)-P(2)$ & $1.839(3)$ \\
\hline$C(20)-C(21)$ & $1.393(4)$ \\
\hline $\mathrm{C}(20)-\mathrm{H}(20)$ & 0.9500 \\
\hline$C(21)-C(22)$ & $1.367(4)$ \\
\hline $\mathrm{C}(21)-\mathrm{H}(21)$ & 0.9500 \\
\hline$C(22)-C(23)$ & $1.380(4)$ \\
\hline $\mathrm{C}(22)-\mathrm{H}(22)$ & 0.9500 \\
\hline$C(23)-C(24)$ & $1.382(4)$ \\
\hline $\mathrm{C}(23)-\mathrm{H}(23)$ & 0.9500 \\
\hline $\mathrm{C}(24)-\mathrm{H}(24)$ & 0.9500 \\
\hline$C(25)-C(30)$ & $1.387(4)$ \\
\hline$C(25)-C(26)$ & $1.390(4)$ \\
\hline$C(25)-P(2)$ & $1.849(3)$ \\
\hline$C(26)-C(27)$ & $1.391(4)$ \\
\hline $\mathrm{C}(26)-\mathrm{H}(26)$ & 0.9500 \\
\hline$C(27)-C(28)$ & $1.373(4)$ \\
\hline
\end{tabular}




\begin{tabular}{|c|c|}
\hline $\mathrm{C}(27)-\mathrm{H}(27)$ & 0.9500 \\
\hline$C(28)-C(29)$ & $1.370(4)$ \\
\hline $\mathrm{C}(28)-\mathrm{H}(28)$ & 0.9500 \\
\hline$C(29)-C(30)$ & $1.384(4)$ \\
\hline $\mathrm{C}(29)-\mathrm{H}(29)$ & 0.9500 \\
\hline $\mathrm{C}(30)-\mathrm{H}(30)$ & 0.9500 \\
\hline$C(31)-C(32)$ & $1.378(4)$ \\
\hline$C(31)-C(36)$ & $1.398(4)$ \\
\hline $\mathrm{C}(31)-\mathrm{P}(2)$ & $1.837(3)$ \\
\hline$C(32)-C(33)$ & $1.389(4)$ \\
\hline $\mathrm{C}(32)-\mathrm{H}(32)$ & 0.9500 \\
\hline$C(33)-C(34)$ & $1.373(4)$ \\
\hline $\mathrm{C}(33)-\mathrm{H}(33)$ & 0.9500 \\
\hline$C(34)-C(35)$ & $1.376(4)$ \\
\hline $\mathrm{C}(34)-\mathrm{H}(34)$ & 0.9500 \\
\hline$C(35)-C(36)$ & $1.377(4)$ \\
\hline $\mathrm{C}(35)-\mathrm{H}(35)$ & 0.9500 \\
\hline $\mathrm{C}(36)-\mathrm{H}(36)$ & 0.9500 \\
\hline$C(37)-C(42)$ & $1.388(4)$ \\
\hline$C(37)-C(38)$ & $1.399(4)$ \\
\hline$C(37)-C(43)$ & $1.471(4)$ \\
\hline$C(38)-C(39)$ & $1.377(4)$ \\
\hline $\mathrm{C}(38)-\mathrm{H}(38)$ & 0.9500 \\
\hline$C(39)-C(40)$ & $1.375(5)$ \\
\hline $\mathrm{C}(39)-\mathrm{H}(39)$ & 0.9500 \\
\hline$C(40)-C(41)$ & $1.373(5)$ \\
\hline $\mathrm{C}(40)-\mathrm{H}(40)$ & 0.9500 \\
\hline$C(41)-C(42)$ & $1.381(4)$ \\
\hline $\mathrm{C}(41)-\mathrm{H}(41)$ & 0.9500 \\
\hline $\mathrm{C}(42)-\mathrm{H}(42)$ & 0.9500 \\
\hline $\mathrm{C}(43)-\mathrm{N}(1)$ & $1.346(3)$ \\
\hline$C(43)-C(44)$ & $1.396(4)$ \\
\hline$C(44)-C(45)$ & $1.352(4)$ \\
\hline $\mathrm{C}(44)-\mathrm{H}(44)$ & 0.9500 \\
\hline $\mathrm{C}(45)-\mathrm{N}(2)$ & $1.362(3)$ \\
\hline$C(45)-C(46)$ & $1.504(4)$ \\
\hline $\mathrm{C}(46)-\mathrm{H}(46 \mathrm{~A})$ & 0.9800 \\
\hline $\mathrm{C}(46)-\mathrm{H}(46 \mathrm{~B})$ & 0.9800 \\
\hline $\mathrm{C}(46)-\mathrm{H}(46 \mathrm{C})$ & 0.9800 \\
\hline$C(47)-C(48)$ & $1.387(4)$ \\
\hline$C(47)-C(52)$ & $1.391(4)$ \\
\hline$C(47)-C(53)$ & $1.475(4)$ \\
\hline$C(48)-C(49)$ & $1.374(4)$ \\
\hline $\mathrm{C}(48)-\mathrm{H}(48)$ & 0.9500 \\
\hline$C(49)-C(50)$ & $1.379(5)$ \\
\hline $\mathrm{C}(49)-\mathrm{H}(49)$ & 0.9500 \\
\hline$C(50)-C(51)$ & $1.382(5)$ \\
\hline $\mathrm{C}(50)-\mathrm{H}(50)$ & 0.9500 \\
\hline$C(51)-C(52)$ & $1.377(4)$ \\
\hline $\mathrm{C}(51)-\mathrm{H}(51)$ & 0.9500 \\
\hline $\mathrm{C}(52)-\mathrm{H}(52)$ & 0.9500 \\
\hline $\mathrm{C}(53)-\mathrm{N}(4)$ & $1.350(3)$ \\
\hline$C(53)-C(54)$ & $1.386(4)$ \\
\hline$C(54)-C(55)$ & $1.366(4)$ \\
\hline $\mathrm{C}(54)-\mathrm{H}(54)$ & 0.9500 \\
\hline$C(55)-N(3)$ & $1.354(3)$ \\
\hline$C(55)-C(56)$ & $1.500(4)$ \\
\hline
\end{tabular}




\begin{tabular}{|c|c|}
\hline$C(56)-H(56 A)$ & 0.9800 \\
\hline $\mathrm{C}(56)-\mathrm{H}(56 \mathrm{~B})$ & 0.9800 \\
\hline $\mathrm{C}(56)-\mathrm{H}(56 \mathrm{C})$ & 0.9800 \\
\hline$C(57)-C(58)$ & $1.383(4)$ \\
\hline$C(57)-C(62)$ & $1.397(4)$ \\
\hline$C(57)-C(63)$ & $1.471(4)$ \\
\hline$C(58)-C(59)$ & $1.376(4)$ \\
\hline $\mathrm{C}(58)-\mathrm{H}(58)$ & 0.9500 \\
\hline$C(59)-C(60)$ & $1.375(4)$ \\
\hline $\mathrm{C}(59)-\mathrm{H}(59)$ & 0.9500 \\
\hline$C(60)-C(61)$ & $1.372(5)$ \\
\hline $\mathrm{C}(60)-\mathrm{H}(60)$ & 0.9500 \\
\hline$C(61)-C(62)$ & $1.389(4)$ \\
\hline $\mathrm{C}(61)-\mathrm{H}(61)$ & 0.9500 \\
\hline $\mathrm{C}(62)-\mathrm{H}(62)$ & 0.9500 \\
\hline$C(63)-N(6)$ & $1.337(3)$ \\
\hline$C(63)-C(64)$ & $1.405(4)$ \\
\hline$C(64)-C(65)$ & $1.378(4)$ \\
\hline $\mathrm{C}(64)-\mathrm{H}(64)$ & 0.9500 \\
\hline$C(65)-N(5)$ & $1.355(3)$ \\
\hline$C(65)-C(66)$ & $1.494(4)$ \\
\hline $\mathrm{C}(66)-\mathrm{H}(66 \mathrm{~A})$ & 0.9800 \\
\hline$C(66)-H(66 B)$ & 0.9800 \\
\hline $\mathrm{C}(66)-\mathrm{H}(66 \mathrm{C})$ & 0.9800 \\
\hline $\mathrm{B}(1)-\mathrm{N}(3)$ & $1.545(4)$ \\
\hline$B(1)-N(5)$ & $1.551(4)$ \\
\hline $\mathrm{B}(1)-\mathrm{N}(2)$ & $1.554(4)$ \\
\hline $\mathrm{B}(1)-\mathrm{H}(1)$ & $1.10(3)$ \\
\hline $\mathrm{N}(1)-\mathrm{N}(2)$ & $1.377(3)$ \\
\hline $\mathrm{N}(1)-\mathrm{Rh}(1)$ & $2.100(2)$ \\
\hline$N(3)-N(4)$ & $1.383(3)$ \\
\hline $\mathrm{N}(4)-\mathrm{Rh}(1)$ & $2.140(2)$ \\
\hline$N(5)-N(6)$ & $1.377(3)$ \\
\hline $\mathrm{P}(1)-\mathrm{Rh}(1)$ & $2.2683(7)$ \\
\hline $\mathrm{P}(2)-\mathrm{Rh}(1)$ & $2.2572(7)$ \\
\hline$C(2)-C(1)-C(6)$ & $118.8(3)$ \\
\hline$C(2)-C(1)-P(1)$ & $119.1(2)$ \\
\hline$C(6)-C(1)-P(1)$ & $121.9(2)$ \\
\hline$C(3)-C(2)-C(1)$ & $120.3(3)$ \\
\hline $\mathrm{C}(3)-\mathrm{C}(2)-\mathrm{H}(2)$ & 119.8 \\
\hline $\mathrm{C}(1)-\mathrm{C}(2)-\mathrm{H}(2)$ & 119.8 \\
\hline$C(4)-C(3)-C(2)$ & $120.8(3)$ \\
\hline $\mathrm{C}(4)-\mathrm{C}(3)-\mathrm{H}(3)$ & 119.6 \\
\hline $\mathrm{C}(2)-\mathrm{C}(3)-\mathrm{H}(3)$ & 119.6 \\
\hline$C(5)-C(4)-C(3)$ & $118.9(3)$ \\
\hline $\mathrm{C}(5)-\mathrm{C}(4)-\mathrm{H}(4)$ & 120.5 \\
\hline $\mathrm{C}(3)-\mathrm{C}(4)-\mathrm{H}(4)$ & 120.5 \\
\hline$C(4)-C(5)-C(6)$ & $121.3(3)$ \\
\hline $\mathrm{C}(4)-\mathrm{C}(5)-\mathrm{H}(5)$ & 119.3 \\
\hline$C(6)-C(5)-H(5)$ & 119.3 \\
\hline$C(5)-C(6)-C(1)$ & $119.8(3)$ \\
\hline $\mathrm{C}(5)-\mathrm{C}(6)-\mathrm{H}(6)$ & 120.1 \\
\hline $\mathrm{C}(1)-\mathrm{C}(6)-\mathrm{H}(6)$ & 120.1 \\
\hline$C(12)-C(7)-C(8)$ & $118.5(2)$ \\
\hline $\mathrm{C}(12)-\mathrm{C}(7)-\mathrm{P}(1)$ & $124.9(2)$ \\
\hline $\mathrm{C}(8)-\mathrm{C}(7)-\mathrm{P}(1)$ & $116.6(2)$ \\
\hline
\end{tabular}




\begin{tabular}{|c|c|}
\hline$C(9)-C(8)-C(7)$ & $120.5(3)$ \\
\hline $\mathrm{C}(9)-\mathrm{C}(8)-\mathrm{H}(8)$ & 119.7 \\
\hline $\mathrm{C}(7)-\mathrm{C}(8)-\mathrm{H}(8)$ & 119.7 \\
\hline$C(8)-C(9)-C(10)$ & $120.3(3)$ \\
\hline $\mathrm{C}(8)-\mathrm{C}(9)-\mathrm{H}(9)$ & 119.8 \\
\hline $\mathrm{C}(10)-\mathrm{C}(9)-\mathrm{H}(9)$ & 119.8 \\
\hline$C(11)-C(10)-C(9)$ & $119.5(3)$ \\
\hline $\mathrm{C}(11)-\mathrm{C}(10)-\mathrm{H}(10)$ & 120.2 \\
\hline $\mathrm{C}(9)-\mathrm{C}(10)-\mathrm{H}(10)$ & 120.2 \\
\hline$C(10)-C(11)-C(12)$ & $120.7(3)$ \\
\hline $\mathrm{C}(10)-\mathrm{C}(11)-\mathrm{H}(11)$ & 119.7 \\
\hline $\mathrm{C}(12)-\mathrm{C}(11)-\mathrm{H}(11)$ & 119.7 \\
\hline$C(11)-C(12)-C(7)$ & $120.4(3)$ \\
\hline $\mathrm{C}(11)-\mathrm{C}(12)-\mathrm{H}(12)$ & 119.8 \\
\hline $\mathrm{C}(7)-\mathrm{C}(12)-\mathrm{H}(12)$ & 119.8 \\
\hline$C(14)-C(13)-C(18)$ & $118.3(3)$ \\
\hline$C(14)-C(13)-P(1)$ & $120.6(2)$ \\
\hline$C(18)-C(13)-P(1)$ & $120.9(2)$ \\
\hline$C(15)-C(14)-C(13)$ & $120.5(3)$ \\
\hline $\mathrm{C}(15)-\mathrm{C}(14)-\mathrm{H}(14)$ & 119.8 \\
\hline $\mathrm{C}(13)-\mathrm{C}(14)-\mathrm{H}(14)$ & 119.8 \\
\hline$C(14)-C(15)-C(16)$ & $120.7(3)$ \\
\hline $\mathrm{C}(14)-\mathrm{C}(15)-\mathrm{H}(15)$ & 119.6 \\
\hline $\mathrm{C}(16)-\mathrm{C}(15)-\mathrm{H}(15)$ & 119.6 \\
\hline$C(15)-C(16)-C(17)$ & $119.5(3)$ \\
\hline $\mathrm{C}(15)-\mathrm{C}(16)-\mathrm{H}(16)$ & 120.2 \\
\hline $\mathrm{C}(17)-\mathrm{C}(16)-\mathrm{H}(16)$ & 120.2 \\
\hline$C(16)-C(17)-C(18)$ & $120.5(3)$ \\
\hline $\mathrm{C}(16)-\mathrm{C}(17)-\mathrm{H}(17)$ & 119.8 \\
\hline $\mathrm{C}(18)-\mathrm{C}(17)-\mathrm{H}(17)$ & 119.8 \\
\hline$C(17)-C(18)-C(13)$ & $120.5(3)$ \\
\hline $\mathrm{C}(17)-\mathrm{C}(18)-\mathrm{H}(18)$ & 119.7 \\
\hline $\mathrm{C}(13)-\mathrm{C}(18)-\mathrm{H}(18)$ & 119.7 \\
\hline$C(20)-C(19)-C(24)$ & $118.7(2)$ \\
\hline$C(20)-C(19)-P(2)$ & $120.2(2)$ \\
\hline$C(24)-C(19)-P(2)$ & $121.0(2)$ \\
\hline$C(19)-C(20)-C(21)$ & $119.8(3)$ \\
\hline $\mathrm{C}(19)-\mathrm{C}(20)-\mathrm{H}(20)$ & 120.1 \\
\hline $\mathrm{C}(21)-\mathrm{C}(20)-\mathrm{H}(20)$ & 120.1 \\
\hline$C(22)-C(21)-C(20)$ & $120.8(3)$ \\
\hline $\mathrm{C}(22)-\mathrm{C}(21)-\mathrm{H}(21)$ & 119.6 \\
\hline $\mathrm{C}(20)-\mathrm{C}(21)-\mathrm{H}(21)$ & 119.6 \\
\hline$C(21)-C(22)-C(23)$ & $119.9(3)$ \\
\hline $\mathrm{C}(21)-\mathrm{C}(22)-\mathrm{H}(22)$ & 120.1 \\
\hline $\mathrm{C}(23)-\mathrm{C}(22)-\mathrm{H}(22)$ & 120.1 \\
\hline$C(22)-C(23)-C(24)$ & $119.9(3)$ \\
\hline $\mathrm{C}(22)-\mathrm{C}(23)-\mathrm{H}(23)$ & 120.0 \\
\hline $\mathrm{C}(24)-\mathrm{C}(23)-\mathrm{H}(23)$ & 120.0 \\
\hline$C(23)-C(24)-C(19)$ & $120.6(3)$ \\
\hline $\mathrm{C}(23)-\mathrm{C}(24)-\mathrm{H}(24)$ & 119.7 \\
\hline $\mathrm{C}(19)-\mathrm{C}(24)-\mathrm{H}(24)$ & 119.7 \\
\hline$C(30)-C(25)-C(26)$ & $117.4(2)$ \\
\hline$C(30)-C(25)-P(2)$ & $116.4(2)$ \\
\hline$C(26)-C(25)-P(2)$ & $126.2(2)$ \\
\hline$C(25)-C(26)-C(27)$ & $120.8(3)$ \\
\hline $\mathrm{C}(25)-\mathrm{C}(26)-\mathrm{H}(26)$ & 119.6 \\
\hline $\mathrm{C}(27)-\mathrm{C}(26)-\mathrm{H}(26)$ & 119.6 \\
\hline
\end{tabular}




\begin{tabular}{|c|c|}
\hline$C(28)-C(27)-C(26)$ & $120.4(3)$ \\
\hline $\mathrm{C}(28)-\mathrm{C}(27)-\mathrm{H}(27)$ & 119.8 \\
\hline $\mathrm{C}(26)-\mathrm{C}(27)-\mathrm{H}(27)$ & 119.8 \\
\hline$C(29)-C(28)-C(27)$ & $119.7(3)$ \\
\hline $\mathrm{C}(29)-\mathrm{C}(28)-\mathrm{H}(28)$ & 120.2 \\
\hline $\mathrm{C}(27)-\mathrm{C}(28)-\mathrm{H}(28)$ & 120.2 \\
\hline$C(28)-C(29)-C(30)$ & $119.9(3)$ \\
\hline $\mathrm{C}(28)-\mathrm{C}(29)-\mathrm{H}(29)$ & 120.0 \\
\hline $\mathrm{C}(30)-\mathrm{C}(29)-\mathrm{H}(29)$ & 120.0 \\
\hline$C(29)-C(30)-C(25)$ & $121.8(3)$ \\
\hline $\mathrm{C}(29)-\mathrm{C}(30)-\mathrm{H}(30)$ & 119.1 \\
\hline $\mathrm{C}(25)-\mathrm{C}(30)-\mathrm{H}(30)$ & 119.1 \\
\hline$C(32)-C(31)-C(36)$ & $118.7(2)$ \\
\hline$C(32)-C(31)-P(2)$ & $124.52(19)$ \\
\hline$C(36)-C(31)-P(2)$ & $116.7(2)$ \\
\hline$C(31)-C(32)-C(33)$ & $120.0(3)$ \\
\hline $\mathrm{C}(31)-\mathrm{C}(32)-\mathrm{H}(32)$ & 120.0 \\
\hline $\mathrm{C}(33)-\mathrm{C}(32)-\mathrm{H}(32)$ & 120.0 \\
\hline$C(34)-C(33)-C(32)$ & $120.7(3)$ \\
\hline $\mathrm{C}(34)-\mathrm{C}(33)-\mathrm{H}(33)$ & 119.6 \\
\hline $\mathrm{C}(32)-\mathrm{C}(33)-\mathrm{H}(33)$ & 119.6 \\
\hline$C(33)-C(34)-C(35)$ & $119.7(3)$ \\
\hline $\mathrm{C}(33)-\mathrm{C}(34)-\mathrm{H}(34)$ & 120.2 \\
\hline $\mathrm{C}(35)-\mathrm{C}(34)-\mathrm{H}(34)$ & 120.2 \\
\hline$C(34)-C(35)-C(36)$ & $120.1(3)$ \\
\hline $\mathrm{C}(34)-\mathrm{C}(35)-\mathrm{H}(35)$ & 120.0 \\
\hline $\mathrm{C}(36)-\mathrm{C}(35)-\mathrm{H}(35)$ & 120.0 \\
\hline$C(35)-C(36)-C(31)$ & $120.7(3)$ \\
\hline $\mathrm{C}(35)-\mathrm{C}(36)-\mathrm{H}(36)$ & 119.7 \\
\hline $\mathrm{C}(31)-\mathrm{C}(36)-\mathrm{H}(36)$ & 119.7 \\
\hline$C(42)-C(37)-C(38)$ & $118.0(3)$ \\
\hline$C(42)-C(37)-C(43)$ & $122.1(3)$ \\
\hline$C(38)-C(37)-C(43)$ & $119.8(3)$ \\
\hline$C(39)-C(38)-C(37)$ & $120.2(3)$ \\
\hline $\mathrm{C}(39)-\mathrm{C}(38)-\mathrm{H}(38)$ & 119.9 \\
\hline $\mathrm{C}(37)-\mathrm{C}(38)-\mathrm{H}(38)$ & 119.9 \\
\hline$C(40)-C(39)-C(38)$ & $121.1(3)$ \\
\hline $\mathrm{C}(40)-\mathrm{C}(39)-\mathrm{H}(39)$ & 119.4 \\
\hline $\mathrm{C}(38)-\mathrm{C}(39)-\mathrm{H}(39)$ & 119.4 \\
\hline$C(41)-C(40)-C(39)$ & $119.2(3)$ \\
\hline $\mathrm{C}(41)-\mathrm{C}(40)-\mathrm{H}(40)$ & 120.4 \\
\hline $\mathrm{C}(39)-\mathrm{C}(40)-\mathrm{H}(40)$ & 120.4 \\
\hline$C(40)-C(41)-C(42)$ & $120.4(3)$ \\
\hline $\mathrm{C}(40)-\mathrm{C}(41)-\mathrm{H}(41)$ & 119.8 \\
\hline $\mathrm{C}(42)-\mathrm{C}(41)-\mathrm{H}(41)$ & 119.8 \\
\hline$C(41)-C(42)-C(37)$ & $121.0(3)$ \\
\hline $\mathrm{C}(41)-\mathrm{C}(42)-\mathrm{H}(42)$ & 119.5 \\
\hline $\mathrm{C}(37)-\mathrm{C}(42)-\mathrm{H}(42)$ & 119.5 \\
\hline$N(1)-C(43)-C(44)$ & $108.9(2)$ \\
\hline$N(1)-C(43)-C(37)$ & $124.3(2)$ \\
\hline$C(44)-C(43)-C(37)$ & $126.6(3)$ \\
\hline$C(45)-C(44)-C(43)$ & $106.8(3)$ \\
\hline $\mathrm{C}(45)-\mathrm{C}(44)-\mathrm{H}(44)$ & 126.6 \\
\hline $\mathrm{C}(43)-\mathrm{C}(44)-\mathrm{H}(44)$ & 126.6 \\
\hline$C(44)-C(45)-N(2)$ & $108.6(2)$ \\
\hline$C(44)-C(45)-C(46)$ & $127.0(3)$ \\
\hline$N(2)-C(45)-C(46)$ & $124.3(3)$ \\
\hline
\end{tabular}




\begin{tabular}{|c|c|}
\hline$C(45)-C(46)-H(46 A)$ & 109.5 \\
\hline $\mathrm{C}(45)-\mathrm{C}(46)-\mathrm{H}(46 \mathrm{~B})$ & 109.5 \\
\hline $\mathrm{H}(46 \mathrm{~A})-\mathrm{C}(46)-\mathrm{H}(46 \mathrm{~B})$ & 109.5 \\
\hline $\mathrm{C}(45)-\mathrm{C}(46)-\mathrm{H}(46 \mathrm{C})$ & 109.5 \\
\hline $\mathrm{H}(46 \mathrm{~A})-\mathrm{C}(46)-\mathrm{H}(46 \mathrm{C})$ & 109.5 \\
\hline $\mathrm{H}(46 \mathrm{~B})-\mathrm{C}(46)-\mathrm{H}(46 \mathrm{C})$ & 109.5 \\
\hline$C(48)-C(47)-C(52)$ & $118.7(3)$ \\
\hline$C(48)-C(47)-C(53)$ & $120.4(3)$ \\
\hline$C(52)-C(47)-C(53)$ & $120.6(3)$ \\
\hline$C(49)-C(48)-C(47)$ & $121.1(3)$ \\
\hline $\mathrm{C}(49)-\mathrm{C}(48)-\mathrm{H}(48)$ & 119.4 \\
\hline $\mathrm{C}(47)-\mathrm{C}(48)-\mathrm{H}(48)$ & 119.4 \\
\hline$C(48)-C(49)-C(50)$ & $119.7(3)$ \\
\hline $\mathrm{C}(48)-\mathrm{C}(49)-\mathrm{H}(49)$ & 120.2 \\
\hline $\mathrm{C}(50)-\mathrm{C}(49)-\mathrm{H}(49)$ & 120.2 \\
\hline$C(49)-C(50)-C(51)$ & $120.0(3)$ \\
\hline $\mathrm{C}(49)-\mathrm{C}(50)-\mathrm{H}(50)$ & 120.0 \\
\hline $\mathrm{C}(51)-\mathrm{C}(50)-\mathrm{H}(50)$ & 120.0 \\
\hline$C(52)-C(51)-C(50)$ & $120.3(3)$ \\
\hline $\mathrm{C}(52)-\mathrm{C}(51)-\mathrm{H}(51)$ & 119.8 \\
\hline $\mathrm{C}(50)-\mathrm{C}(51)-\mathrm{H}(51)$ & 119.8 \\
\hline$C(51)-C(52)-C(47)$ & $120.2(3)$ \\
\hline $\mathrm{C}(51)-\mathrm{C}(52)-\mathrm{H}(52)$ & 119.9 \\
\hline $\mathrm{C}(47)-\mathrm{C}(52)-\mathrm{H}(52)$ & 119.9 \\
\hline$N(4)-C(53)-C(54)$ & $110.1(2)$ \\
\hline$N(4)-C(53)-C(47)$ & $122.9(2)$ \\
\hline$C(54)-C(53)-C(47)$ & $126.6(3)$ \\
\hline$C(55)-C(54)-C(53)$ & $106.1(3)$ \\
\hline $\mathrm{C}(55)-\mathrm{C}(54)-\mathrm{H}(54)$ & 126.9 \\
\hline $\mathrm{C}(53)-\mathrm{C}(54)-\mathrm{H}(54)$ & 126.9 \\
\hline$N(3)-C(55)-C(54)$ & $108.5(3)$ \\
\hline $\mathrm{N}(3)-\mathrm{C}(55)-\mathrm{C}(56)$ & $121.9(3)$ \\
\hline$C(54)-C(55)-C(56)$ & $129.5(3)$ \\
\hline $\mathrm{C}(55)-\mathrm{C}(56)-\mathrm{H}(56 \mathrm{~A})$ & 109.5 \\
\hline$C(55)-C(56)-H(56 B)$ & 109.5 \\
\hline $\mathrm{H}(56 \mathrm{~A})-\mathrm{C}(56)-\mathrm{H}(56 \mathrm{~B})$ & 109.5 \\
\hline$C(55)-C(56)-H(56 C)$ & 109.5 \\
\hline $\mathrm{H}(56 \mathrm{~A})-\mathrm{C}(56)-\mathrm{H}(56 \mathrm{C})$ & 109.5 \\
\hline $\mathrm{H}(56 \mathrm{~B})-\mathrm{C}(56)-\mathrm{H}(56 \mathrm{C})$ & 109.5 \\
\hline$C(58)-C(57)-C(62)$ & $117.6(3)$ \\
\hline$C(58)-C(57)-C(63)$ & $122.0(3)$ \\
\hline$C(62)-C(57)-C(63)$ & $120.4(3)$ \\
\hline$C(59)-C(58)-C(57)$ & $121.7(3)$ \\
\hline $\mathrm{C}(59)-\mathrm{C}(58)-\mathrm{H}(58)$ & 119.1 \\
\hline $\mathrm{C}(57)-\mathrm{C}(58)-\mathrm{H}(58)$ & 119.1 \\
\hline$C(60)-C(59)-C(58)$ & $120.2(3)$ \\
\hline $\mathrm{C}(60)-\mathrm{C}(59)-\mathrm{H}(59)$ & 119.9 \\
\hline $\mathrm{C}(58)-\mathrm{C}(59)-\mathrm{H}(59)$ & 119.9 \\
\hline$C(61)-C(60)-C(59)$ & $119.5(3)$ \\
\hline $\mathrm{C}(61)-\mathrm{C}(60)-\mathrm{H}(60)$ & 120.3 \\
\hline$C(59)-C(60)-H(60)$ & 120.3 \\
\hline$C(60)-C(61)-C(62)$ & $120.5(3)$ \\
\hline $\mathrm{C}(60)-\mathrm{C}(61)-\mathrm{H}(61)$ & 119.7 \\
\hline $\mathrm{C}(62)-\mathrm{C}(61)-\mathrm{H}(61)$ & 119.7 \\
\hline $\mathrm{C}(61)-\mathrm{C}(62)-\mathrm{C}(57)$ & $120.5(3)$ \\
\hline $\mathrm{C}(61)-\mathrm{C}(62)-\mathrm{H}(62)$ & 119.8 \\
\hline $\mathrm{C}(57)-\mathrm{C}(62)-\mathrm{H}(62)$ & 119.8 \\
\hline
\end{tabular}




\begin{tabular}{|c|c|}
\hline$N(6)-C(63)-C(64)$ & $110.8(3)$ \\
\hline$N(6)-C(63)-C(57)$ & $120.9(3)$ \\
\hline$C(64)-C(63)-C(57)$ & $128.3(3)$ \\
\hline$C(65)-C(64)-C(63)$ & $105.3(3)$ \\
\hline $\mathrm{C}(65)-\mathrm{C}(64)-\mathrm{H}(64)$ & 127.3 \\
\hline $\mathrm{C}(63)-\mathrm{C}(64)-\mathrm{H}(64)$ & 127.3 \\
\hline$N(5)-C(65)-C(64)$ & $107.7(3)$ \\
\hline$N(5)-C(65)-C(66)$ & $123.9(3)$ \\
\hline$C(64)-C(65)-C(66)$ & $128.3(3)$ \\
\hline$C(65)-C(66)-H(66 A)$ & 109.5 \\
\hline$C(65)-C(66)-H(66 B)$ & 109.5 \\
\hline $\mathrm{H}(66 \mathrm{~A})-\mathrm{C}(66)-\mathrm{H}(66 \mathrm{~B})$ & 109.5 \\
\hline $\mathrm{C}(65)-\mathrm{C}(66)-\mathrm{H}(66 \mathrm{C})$ & 109.5 \\
\hline $\mathrm{H}(66 \mathrm{~A})-\mathrm{C}(66)-\mathrm{H}(66 \mathrm{C})$ & 109.5 \\
\hline $\mathrm{H}(66 \mathrm{~B})-\mathrm{C}(66)-\mathrm{H}(66 \mathrm{C})$ & 109.5 \\
\hline $\mathrm{N}(3)-\mathrm{B}(1)-\mathrm{N}(5)$ & $110.9(2)$ \\
\hline$N(3)-B(1)-N(2)$ & $108.6(2)$ \\
\hline$N(5)-B(1)-N(2)$ & $116.2(2)$ \\
\hline $\mathrm{N}(3)-\mathrm{B}(1)-\mathrm{H}(1)$ & $108.9(14)$ \\
\hline $\mathrm{N}(5)-\mathrm{B}(1)-\mathrm{H}(1)$ & $104.0(14)$ \\
\hline $\mathrm{N}(2)-\mathrm{B}(1)-\mathrm{H}(1)$ & $108.0(14)$ \\
\hline $\mathrm{C}(43)-\mathrm{N}(1)-\mathrm{N}(2)$ & $107.1(2)$ \\
\hline $\mathrm{C}(43)-\mathrm{N}(1)-\mathrm{Rh}(1)$ & $135.38(17)$ \\
\hline$N(2)-N(1)-R h(1)$ & $117.27(16)$ \\
\hline $\mathrm{C}(45)-\mathrm{N}(2)-\mathrm{N}(1)$ & $108.5(2)$ \\
\hline $\mathrm{C}(45)-\mathrm{N}(2)-\mathrm{B}(1)$ & $126.8(2)$ \\
\hline $\mathrm{N}(1)-\mathrm{N}(2)-\mathrm{B}(1)$ & $121.3(2)$ \\
\hline $\mathrm{C}(55)-\mathrm{N}(3)-\mathrm{N}(4)$ & $109.2(2)$ \\
\hline $\mathrm{C}(55)-\mathrm{N}(3)-\mathrm{B}(1)$ & $126.5(2)$ \\
\hline$N(4)-N(3)-B(1)$ & $122.7(2)$ \\
\hline $\mathrm{C}(53)-\mathrm{N}(4)-\mathrm{N}(3)$ & $106.0(2)$ \\
\hline $\mathrm{C}(53)-\mathrm{N}(4)-\mathrm{Rh}(1)$ & $139.10(19)$ \\
\hline$N(3)-N(4)-R h(1)$ & $112.63(15)$ \\
\hline$C(65)-N(5)-N(6)$ & $110.5(2)$ \\
\hline $\mathrm{C}(65)-\mathrm{N}(5)-\mathrm{B}(1)$ & $127.1(2)$ \\
\hline$N(6)-N(5)-B(1)$ & $118.8(2)$ \\
\hline $\mathrm{C}(63)-\mathrm{N}(6)-\mathrm{N}(5)$ & $105.7(2)$ \\
\hline $\mathrm{C}(1)-\mathrm{P}(1)-\mathrm{C}(7)$ & $101.33(12)$ \\
\hline$C(1)-P(1)-C(13)$ & $98.93(12)$ \\
\hline$C(7)-P(1)-C(13)$ & $102.87(12)$ \\
\hline $\mathrm{C}(1)-\mathrm{P}(1)-\mathrm{Rh}(1)$ & $116.79(9)$ \\
\hline $\mathrm{C}(7)-\mathrm{P}(1)-\mathrm{Rh}(1)$ & $114.23(9)$ \\
\hline $\mathrm{C}(13)-\mathrm{P}(1)-\mathrm{Rh}(1)$ & $119.87(8)$ \\
\hline$C(31)-P(2)-C(19)$ & $96.84(11)$ \\
\hline$C(31)-P(2)-C(25)$ & $102.10(12)$ \\
\hline$C(19)-P(2)-C(25)$ & $104.62(12)$ \\
\hline $\mathrm{C}(31)-\mathrm{P}(2)-\mathrm{Rh}(1)$ & $112.89(8)$ \\
\hline $\mathrm{C}(19)-\mathrm{P}(2)-\mathrm{Rh}(1)$ & $118.12(9)$ \\
\hline $\mathrm{C}(25)-\mathrm{P}(2)-\mathrm{Rh}(1)$ & $118.98(8)$ \\
\hline $\mathrm{N}(1)-\mathrm{Rh}(1)-\mathrm{N}(4)$ & $77.49(8)$ \\
\hline $\mathrm{N}(1)-\mathrm{Rh}(1)-\mathrm{P}(2)$ & $92.42(6)$ \\
\hline$N(4)-R h(1)-P(2)$ & $167.66(6)$ \\
\hline $\mathrm{N}(1)-\mathrm{Rh}(1)-\mathrm{P}(1)$ & $173.25(6)$ \\
\hline$N(4)-\operatorname{Rh}(1)-P(1)$ & $96.18(6)$ \\
\hline$P(2)-R h(1)-P(1)$ & $94.15(3)$ \\
\hline
\end{tabular}

Symmetry transformations used to generate equivalent atoms: 
Table S22. Anisotropic displacement parameters ( $A^{\wedge} 2 \times 10^{\wedge} 3$ ) for complex 5. The anisotropic displacement factor exponent takes the form:

$-2 \mathrm{pi}^{\wedge} 2\left[\mathrm{~h}^{\wedge} 2 \mathrm{a}^{\star \wedge} 2 \mathrm{U11}+\ldots+2 \mathrm{~h} \mathrm{k} \mathrm{a}^{\star} \mathrm{b}^{\star} \mathrm{U} 12\right.$ ]

\begin{tabular}{|c|c|c|c|c|c|c|}
\hline & $\mathrm{U} 11$ & $\mathrm{U} 22$ & U33 & $\mathrm{U} 23$ & $\mathrm{U} 13$ & $\mathrm{U} 12$ \\
\hline$C(1)$ & $32(2)$ & $18(2)$ & $28(1)$ & $1(1)$ & $11(1)$ & $1(1)$ \\
\hline C (2) & $41(2)$ & $23(2)$ & $35(2)$ & $1(1)$ & $9(1)$ & $5(1)$ \\
\hline$C(3)$ & $44(2)$ & $36(2)$ & $50(2)$ & $6(2)$ & $14(2)$ & $14(2)$ \\
\hline$C(4)$ & $60(2)$ & $24(2)$ & $58(2)$ & $-2(2)$ & $32(2)$ & $9(2)$ \\
\hline$C(5)$ & $58(2)$ & $31(2)$ & $44(2)$ & $-14(2)$ & $21(2)$ & $-4(2)$ \\
\hline$C(6)$ & $40(2)$ & $30(2)$ & $36(2)$ & $-7(1)$ & $11(1)$ & $-5(2)$ \\
\hline$C(7)$ & $30(2)$ & $19(2)$ & $24(1)$ & $-4(1)$ & $1(1)$ & $1(1)$ \\
\hline$C(8)$ & $33(2)$ & $25(2)$ & $37(2)$ & $2(1)$ & $-1(1)$ & $-8(1)$ \\
\hline$C(9)$ & $47(2)$ & $36(2)$ & $40(2)$ & $12(1)$ & $-6(2)$ & $-4(2)$ \\
\hline$C(10)$ & $49(2)$ & $37(2)$ & $38(2)$ & $6(2)$ & $-12(2)$ & $3(2)$ \\
\hline C (11) & $38(2)$ & $31(2)$ & $41(2)$ & $-3(1)$ & $-10(1)$ & $-10(2)$ \\
\hline C (12) & $41(2)$ & $21(2)$ & $30(2)$ & $-2(1)$ & $0(1)$ & $-5(1)$ \\
\hline C (13) & $30(2)$ & $19(2)$ & $22(1)$ & $-5(1)$ & $4(1)$ & $-7(1)$ \\
\hline C (14) & $36(2)$ & $23(2)$ & $31(2)$ & $-1(1)$ & $3(1)$ & $-1(1)$ \\
\hline C (15) & $54(2)$ & $28(2)$ & $38(2)$ & $4(1)$ & $3(2)$ & $-9(2)$ \\
\hline$C(16)$ & $56(2)$ & $33(2)$ & $31(2)$ & $0(1)$ & $8(1)$ & $-21(2)$ \\
\hline C (17) & $35(2)$ & $40(2)$ & $35(2)$ & $-10(1)$ & $10(1)$ & $-16(2)$ \\
\hline$C(18)$ & $32(2)$ & $26(2)$ & $29(2)$ & $-6(1)$ & $4(1)$ & $-5(1)$ \\
\hline C (19) & $24(1)$ & $20(2)$ & $23(1)$ & $-3(1)$ & $-1(1)$ & $-6(1)$ \\
\hline$C(20)$ & $30(2)$ & $21(2)$ & $25(1)$ & $-4(1)$ & $-5(1)$ & $-2(1)$ \\
\hline$C(21)$ & $43(2)$ & $24(2)$ & $35(2)$ & $2(1)$ & $-9(1)$ & $-2(1)$ \\
\hline C (22) & $44(2)$ & $39(2)$ & $27(2)$ & $8(1)$ & $-2(1)$ & $-10(2)$ \\
\hline$C(23)$ & $35(2)$ & $48(2)$ & $27(2)$ & $-1(1)$ & $6(1)$ & $-4(2)$ \\
\hline C (24) & $33(2)$ & $29(2)$ & $27(2)$ & $-2(1)$ & $2(1)$ & $2(1)$ \\
\hline C (25) & $23(1)$ & $18(2)$ & $25(1)$ & $-8(1)$ & $1(1)$ & $3(1)$ \\
\hline$C(26)$ & $26(2)$ & $40(2)$ & $34(2)$ & $0(1)$ & $4(1)$ & $1(1)$ \\
\hline C (27) & $24(2)$ & $47(2)$ & $49(2)$ & $-9(2)$ & $3(1)$ & $3(2)$ \\
\hline C (28) & $33(2)$ & $32(2)$ & $43(2)$ & $-9(1)$ & $-11(1)$ & $9(1)$ \\
\hline C (29) & $44(2)$ & $31(2)$ & $32(2)$ & $0(1)$ & $-8(1)$ & $6(2)$ \\
\hline$C(30)$ & $31(2)$ & $28(2)$ & $32(2)$ & $0(1)$ & $2(1)$ & $2(1)$ \\
\hline$C(31)$ & $25(1)$ & $20(2)$ & $22(1)$ & $-3(1)$ & $8(1)$ & $-3(1)$ \\
\hline C (32) & $34(2)$ & $24(2)$ & $30(2)$ & $-3(1)$ & $-2(1)$ & $1(1)$ \\
\hline C (33) & $51(2)$ & $21(2)$ & $39(2)$ & $-2(1)$ & $5(2)$ & $4(2)$ \\
\hline C (34) & $48(2)$ & $30(2)$ & $30(2)$ & $-11(1)$ & $6(1)$ & $-8(2)$ \\
\hline$C(35)$ & $37(2)$ & $38(2)$ & $34(2)$ & $-7(1)$ & $-5(1)$ & $-5(2)$ \\
\hline$C(36)$ & $31(2)$ & $27(2)$ & $34(2)$ & $-3(1)$ & $-1(1)$ & $1(1)$ \\
\hline$C(37)$ & $25(2)$ & $23(2)$ & $45(2)$ & $3(1)$ & $14(1)$ & $-5(1)$ \\
\hline C (38) & $44(2)$ & $20(2)$ & $55(2)$ & $2(2)$ & $17(2)$ & $1(2)$ \\
\hline C (39) & $50(2)$ & $22(2)$ & $86(3)$ & $15(2)$ & $27(2)$ & $10(2)$ \\
\hline$C(40)$ & $38(2)$ & $44(2)$ & $75(3)$ & $31(2)$ & $8(2)$ & $6(2)$ \\
\hline$C(41)$ & $39(2)$ & $42(2)$ & $57(2)$ & $22(2)$ & $-2(2)$ & $-4(2)$ \\
\hline C (42) & $32(2)$ & $25(2)$ & $47(2)$ & $8(1)$ & $6(1)$ & $-4(1)$ \\
\hline$C(43)$ & $28(2)$ & $20(2)$ & $34(2)$ & $-4(1)$ & $10(1)$ & $-3(1)$ \\
\hline$C(44)$ & $32(2)$ & $25(2)$ & $40(2)$ & $-8(1)$ & $9(1)$ & $-9(1)$ \\
\hline C (45) & $28(2)$ & $36(2)$ & $34(2)$ & $-7(1)$ & $5(1)$ & $-13(1)$ \\
\hline$C(46)$ & $37(2)$ & $50(2)$ & $46(2)$ & $-9(2)$ & $1(1)$ & $-18(2)$ \\
\hline C (47) & $29(2)$ & $33(2)$ & $33(2)$ & $-1(1)$ & $13(1)$ & $-7(1)$ \\
\hline$C(48)$ & $32(2)$ & $36(2)$ & $44(2)$ & $2(2)$ & $5(1)$ & $-7(2)$ \\
\hline
\end{tabular}




\begin{tabular}{|c|c|c|c|c|c|c|}
\hline C (49) & $49(2)$ & $49(2)$ & $49(2)$ & $16(2)$ & $3(2)$ & $-6(2)$ \\
\hline$C(50)$ & $67(3)$ & $82(3)$ & $35(2)$ & $13(2)$ & $-2(2)$ & $-7(2)$ \\
\hline$C(51)$ & $73(3)$ & $74(3)$ & $37(2)$ & $-10(2)$ & $8(2)$ & $-11(2)$ \\
\hline$C(52)$ & $51(2)$ & $40(2)$ & $40(2)$ & $-5(2)$ & $15(2)$ & $-5(2)$ \\
\hline$C(53)$ & $29(2)$ & $22(2)$ & $37(2)$ & $-1(1)$ & 11 (1) & $-4(1)$ \\
\hline$C(54)$ & $36(2)$ & $35(2)$ & $43(2)$ & $1(1)$ & $19(1)$ & $3(2)$ \\
\hline$C(55)$ & $28(2)$ & $31(2)$ & $48(2)$ & $6(1)$ & $9(1)$ & $5(1)$ \\
\hline$C(56)$ & $35(2)$ & $71(3)$ & $61(2)$ & $6(2)$ & $10(2)$ & $22(2)$ \\
\hline$C(57)$ & $36(2)$ & $30(2)$ & $33(2)$ & $8(1)$ & $2(1)$ & $7(2)$ \\
\hline$C(58)$ & $50(2)$ & $33(2)$ & $37(2)$ & $9(1)$ & $9(2)$ & $5(2)$ \\
\hline$C(59)$ & $71(3)$ & $34(2)$ & $44(2)$ & $0(2)$ & $13(2)$ & $6(2)$ \\
\hline$C(60)$ & $72(3)$ & $27(2)$ & $56(2)$ & $8(2)$ & $7(2)$ & $3(2)$ \\
\hline$C(61)$ & $70(3)$ & $39(2)$ & $51(2)$ & $19(2)$ & $19(2)$ & $4(2)$ \\
\hline$C(62)$ & $62(2)$ & $38(2)$ & $40(2)$ & $8(2)$ & $12(2)$ & $10(2)$ \\
\hline$C(63)$ & $32(2)$ & $31(2)$ & $32(2)$ & $5(1)$ & $3(1)$ & $11(1)$ \\
\hline$C(64)$ & $43(2)$ & $38(2)$ & $29(2)$ & $3(1)$ & $3(1)$ & $7(2)$ \\
\hline$C(65)$ & $36(2)$ & $34(2)$ & $30(2)$ & $-1(1)$ & $-1(1)$ & $8(1)$ \\
\hline$C(66)$ & $50(2)$ & $43(2)$ & $35(2)$ & $-2(2)$ & $-1(1)$ & $-4(2)$ \\
\hline$B(1)$ & $24(2)$ & $31(2)$ & $36(2)$ & $2(2)$ & $-1(1)$ & $-2(2)$ \\
\hline $\mathrm{N}(1)$ & $18(1)$ & $23(1)$ & $29(1)$ & $-1(1)$ & $2(1)$ & $-1(1)$ \\
\hline$N(2)$ & $20(1)$ & $28(1)$ & 31 (1) & $-3(1)$ & $2(1)$ & $-4(1)$ \\
\hline$N(3)$ & $21(1)$ & $28(1)$ & $35(1)$ & $3(1)$ & $6(1)$ & $4(1)$ \\
\hline$N(4)$ & $23(1)$ & $21(1)$ & $31(1)$ & $-1(1)$ & $8(1)$ & $-1(1)$ \\
\hline$N(5)$ & $29(1)$ & $29(1)$ & $30(1)$ & $1(1)$ & $-1(1)$ & $2(1)$ \\
\hline$N(6)$ & $32(1)$ & $28(1)$ & 31 (1) & $3(1)$ & $2(1)$ & $4(1)$ \\
\hline $\mathrm{P}(1)$ & $23(1)$ & $17(1)$ & $25(1)$ & $-2(1)$ & $4(1)$ & $-1(1)$ \\
\hline$P(2)$ & $20(1)$ & $18(1)$ & $23(1)$ & $-2(1)$ & $1(1)$ & $0(1)$ \\
\hline $\mathrm{Rh}(1)$ & $19(1)$ & $16(1)$ & $25(1)$ & $-2(1)$ & $3(1)$ & $-1(1)$ \\
\hline
\end{tabular}


Table S23. Hydrogen coordinates $\left(x 1^{\wedge} 4\right)$ and isotropic displacement parameters $\left(A^{\wedge} 2 \times 10^{\wedge} 3\right)$ for complex 5 .

\begin{tabular}{|c|c|c|c|c|}
\hline & $x$ & Y & $z$ & $\mathrm{U}(\mathrm{eq})$ \\
\hline $\mathrm{H}(2)$ & 6398 & 2086 & 3034 & 40 \\
\hline $\mathrm{H}(3)$ & 7313 & 989 & 2731 & 52 \\
\hline $\mathrm{H}(4)$ & 6887 & 260 & 2055 & 56 \\
\hline $\mathrm{H}(5)$ & 5539 & 645 & 1684 & 53 \\
\hline $\mathrm{H}(6)$ & 4616 & 1748 & 1974 & 42 \\
\hline $\mathrm{H}(8)$ & 4670 & 4216 & 2149 & 38 \\
\hline $\mathrm{H}(9)$ & 3800 & 4637 & 1515 & 49 \\
\hline $\mathrm{H}(10)$ & 2472 & 3866 & 1324 & 50 \\
\hline $\mathrm{H}(11)$ & 2072 & 2623 & 1745 & 44 \\
\hline $\mathrm{H}(12)$ & 2967 & 2152 & 2364 & 37 \\
\hline $\mathrm{H}(14)$ & 4980 & 1095 & 3239 & 36 \\
\hline $\mathrm{H}(15)$ & 4179 & 163 & 3708 & 48 \\
\hline $\mathrm{H}(16)$ & 2718 & 529 & 3892 & 48 \\
\hline $\mathrm{H}(17)$ & 2040 & 1817 & 3584 & 44 \\
\hline $\mathrm{H}(18)$ & 2833 & 2769 & 3118 & 35 \\
\hline $\mathrm{H}(20)$ & 4856 & 2688 & 3934 & 31 \\
\hline $\mathrm{H}(21)$ & 4494 & 1712 & 4523 & 41 \\
\hline $\mathrm{H}(22)$ & 3326 & 2047 & 4989 & 44 \\
\hline $\mathrm{H}(23)$ & 2570 & 3416 & 4908 & 44 \\
\hline $\mathrm{H}(24)$ & 2945 & 4415 & 4336 & 36 \\
\hline $\mathrm{H}(26)$ & 2166 & 4025 & 3728 & 40 \\
\hline $\mathrm{H}(27)$ & 847 & 4336 & 3304 & 48 \\
\hline $\mathrm{H}(28)$ & 906 & 5034 & 2600 & 43 \\
\hline $\mathrm{H}(29)$ & 2288 & 5397 & 2310 & 43 \\
\hline $\mathrm{H}(30)$ & 3604 & 5097 & 2732 & 36 \\
\hline $\mathrm{H}(32)$ & 3385 & 6224 & 3566 & 35 \\
\hline $\mathrm{H}(33)$ & 3693 & 7510 & 4000 & 44 \\
\hline $\mathrm{H}(34)$ & 4785 & 7481 & 4583 & 43 \\
\hline $\mathrm{H}(35)$ & 5513 & 6134 & 4769 & 44 \\
\hline $\mathrm{H}(36)$ & 5197 & 4840 & 4349 & 37 \\
\hline $\mathrm{H}(38)$ & 5486 & 7734 & 3385 & 47 \\
\hline $\mathrm{H}(39)$ & 4557 & 8509 & 2871 & 63 \\
\hline $\mathrm{H}(40)$ & 3876 & 7775 & 2251 & 63 \\
\hline $\mathrm{H}(41)$ & 4188 & 6261 & 2127 & 55 \\
\hline $\mathrm{H}(42)$ & 5126 & 5471 & 2633 & 42 \\
\hline $\mathrm{H}(44)$ & 6871 & 7053 & 3667 & 39 \\
\hline $\mathrm{H}(46 \mathrm{~A})$ & 8238 & 6444 & 4155 & 66 \\
\hline $\mathrm{H}(46 \mathrm{~B})$ & 8548 & 5476 & 3979 & 66 \\
\hline $\mathrm{H}(46 \mathrm{C})$ & 7963 & 5551 & 4421 & 66 \\
\hline $\mathrm{H}(48)$ & 6341 & 5180 & 2192 & 45 \\
\hline $\mathrm{H}(49)$ & 5804 & 5586 & 1471 & 59 \\
\hline $\mathrm{H}(50)$ & 5691 & 4505 & 897 & 74 \\
\hline $\mathrm{H}(51)$ & 6140 & 3026 & 1046 & 74 \\
\hline $\mathrm{H}(52)$ & 6716 & 2626 & 1762 & 52 \\
\hline $\mathrm{H}(54)$ & 8252 & 3063 & 2354 & 45 \\
\hline $\mathrm{H}(56 \mathrm{~A})$ & 9147 & 3498 & 3436 & 83 \\
\hline $\mathrm{H}(56 \mathrm{~B})$ & 9213 & 2626 & 3120 & 83 \\
\hline $\mathrm{H}(56 \mathrm{C})$ & 8625 & 2595 & 3562 & 83 \\
\hline
\end{tabular}




\begin{tabular}{llrll} 
H (58) & 6846 & 777 & 3562 & 48 \\
H (59) & 6606 & -759 & 3503 & 60 \\
H (60) & 5973 & -1546 & 4095 & 62 \\
H 61$)$ & 5590 & -782 & 4747 & 64 \\
H $(62)$ & 5860 & 762 & 4818 & 56 \\
H 64$)$ & 6503 & 2243 & 4934 & 44 \\
H $(66 \mathrm{~A})$ & 7586 & 4242 & 4789 & 64 \\
H $(66 \mathrm{~B})$ & 6721 & 4030 & 5077 & 64 \\
$\mathrm{H}(66 \mathrm{C})$ & 6627 & 4646 & 4631 & 64 \\
$\mathrm{H}(1)$ & $7923(18)$ & $4055(17)$ & $3870(8)$ & $34(8)$ \\
\hline
\end{tabular}


Table s24. Torsion angles [deg] for complex 5.

\begin{tabular}{|c|c|}
\hline$C(6)-C(1)-C(2)-C(3)$ & $0.4(4)$ \\
\hline$P(1)-C(1)-C(2)-C(3)$ & $175.4(2)$ \\
\hline$C(1)-C(2)-C(3)-C(4)$ & $-0.4(4)$ \\
\hline$C(2)-C(3)-C(4)-C(5)$ & $0.1(5)$ \\
\hline$C(3)-C(4)-C(5)-C(6)$ & $0.2(5)$ \\
\hline$C(4)-C(5)-C(6)-C(1)$ & $-0.3(5)$ \\
\hline$C(2)-C(1)-C(6)-C(5)$ & $0.0(4)$ \\
\hline$P(1)-C(1)-C(6)-C(5)$ & $-174.9(2)$ \\
\hline$C(12)-C(7)-C(8)-C(9)$ & $0.8(4)$ \\
\hline$P(1)-C(7)-C(8)-C(9)$ & $177.7(2)$ \\
\hline$C(7)-C(8)-C(9)-C(10)$ & $1.4(5)$ \\
\hline$C(8)-C(9)-C(10)-C(11)$ & $-2.1(5)$ \\
\hline$C(9)-C(10)-C(11)-C(12)$ & $0.6(5)$ \\
\hline$C(10)-C(11)-C(12)-C(7)$ & $1.5(5)$ \\
\hline$C(8)-C(7)-C(12)-C(11)$ & $-2.2(4)$ \\
\hline $\mathrm{P}(1)-\mathrm{C}(7)-\mathrm{C}(12)-\mathrm{C}(11)$ & $-178.9(2)$ \\
\hline$C(18)-C(13)-C(14)-C(15)$ & $-1.4(4)$ \\
\hline$P(1)-C(13)-C(14)-C(15)$ & $174.1(2)$ \\
\hline$C(13)-C(14)-C(15)-C(16)$ & $0.3(4)$ \\
\hline$C(14)-C(15)-C(16)-C(17)$ & $1.1(4)$ \\
\hline$C(15)-C(16)-C(17)-C(18)$ & $-1.4(4)$ \\
\hline$C(16)-C(17)-C(18)-C(13)$ & $0.3(4)$ \\
\hline$C(14)-C(13)-C(18)-C(17)$ & $1.1(4)$ \\
\hline$P(1)-C(13)-C(18)-C(17)$ & $-174.4(2)$ \\
\hline$C(24)-C(19)-C(20)-C(21)$ & $5.2(4)$ \\
\hline$P(2)-C(19)-C(20)-C(21)$ & $-177.17(19)$ \\
\hline$C(19)-C(20)-C(21)-C(22)$ & $-0.8(4)$ \\
\hline$C(20)-C(21)-C(22)-C(23)$ & $-2.9(4)$ \\
\hline$C(21)-C(22)-C(23)-C(24)$ & $2.0(4)$ \\
\hline$C(22)-C(23)-C(24)-C(19)$ & $2.5(4)$ \\
\hline$C(20)-C(19)-C(24)-C(23)$ & $-6.1(4)$ \\
\hline$P(2)-C(19)-C(24)-C(23)$ & $176.3(2)$ \\
\hline$C(30)-C(25)-C(26)-C(27)$ & $-1.1(4)$ \\
\hline$P(2)-C(25)-C(26)-C(27)$ & $178.9(2)$ \\
\hline$C(25)-C(26)-C(27)-C(28)$ & $0.3(5)$ \\
\hline$C(26)-C(27)-C(28)-C(29)$ & $0.7(5)$ \\
\hline$C(27)-C(28)-C(29)-C(30)$ & $-0.9(5)$ \\
\hline$C(28)-C(29)-C(30)-C(25)$ & $0.0(4)$ \\
\hline$C(26)-C(25)-C(30)-C(29)$ & $1.0(4)$ \\
\hline$P(2)-C(25)-C(30)-C(29)$ & $-179.1(2)$ \\
\hline$C(36)-C(31)-C(32)-C(33)$ & $1.5(4)$ \\
\hline$P(2)-C(31)-C(32)-C(33)$ & $-176.7(2)$ \\
\hline$C(31)-C(32)-C(33)-C(34)$ & $0.7(4)$ \\
\hline$C(32)-C(33)-C(34)-C(35)$ & $-2.3(5)$ \\
\hline$C(33)-C(34)-C(35)-C(36)$ & $1.6(5)$ \\
\hline$C(34)-C(35)-C(36)-C(31)$ & $0.6(4)$ \\
\hline$C(32)-C(31)-C(36)-C(35)$ & $-2.2(4)$ \\
\hline$P(2)-C(31)-C(36)-C(35)$ & $176.2(2)$ \\
\hline$C(42)-C(37)-C(38)-C(39)$ & $-0.1(4)$ \\
\hline$C(43)-C(37)-C(38)-C(39)$ & $176.8(3)$ \\
\hline$C(37)-C(38)-C(39)-C(40)$ & $1.0(5)$ \\
\hline$C(38)-C(39)-C(40)-C(41)$ & $-1.5(5)$ \\
\hline$C(39)-C(40)-C(41)-C(42)$ & $1.2(5)$ \\
\hline
\end{tabular}




\begin{tabular}{|c|c|}
\hline$C(40)-C(41)-C(42)-C(37)$ & $-0.4(5)$ \\
\hline$C(38)-C(37)-C(42)-C(41)$ & $-0.2(4)$ \\
\hline$C(43)-C(37)-C(42)-C(41)$ & $-177.0(3)$ \\
\hline $\mathrm{C}(42)-\mathrm{C}(37)-\mathrm{C}(43)-\mathrm{N}(1)$ & $-23.9(4)$ \\
\hline $\mathrm{C}(38)-\mathrm{C}(37)-\mathrm{C}(43)-\mathrm{N}(1)$ & $159.3(3)$ \\
\hline$C(42)-C(37)-C(43)-C(44)$ & $150.6(3)$ \\
\hline$C(38)-C(37)-C(43)-C(44)$ & $-26.3(4)$ \\
\hline$N(1)-C(43)-C(44)-C(45)$ & $2.7(3)$ \\
\hline$C(37)-C(43)-C(44)-C(45)$ & $-172.5(3)$ \\
\hline $\mathrm{C}(43)-\mathrm{C}(44)-\mathrm{C}(45)-\mathrm{N}(2)$ & $-2.0(3)$ \\
\hline$C(43)-C(44)-C(45)-C(46)$ & $177.1(3)$ \\
\hline$C(52)-C(47)-C(48)-C(49)$ & $0.2(4)$ \\
\hline$C(53)-C(47)-C(48)-C(49)$ & $173.8(3)$ \\
\hline$C(47)-C(48)-C(49)-C(50)$ & $0.7(5)$ \\
\hline$C(48)-C(49)-C(50)-C(51)$ & $-0.6(6)$ \\
\hline$C(49)-C(50)-C(51)-C(52)$ & $-0.4(6)$ \\
\hline$C(50)-C(51)-C(52)-C(47)$ & $1.3(5)$ \\
\hline$C(48)-C(47)-C(52)-C(51)$ & $-1.2(5)$ \\
\hline$C(53)-C(47)-C(52)-C(51)$ & $-174.7(3)$ \\
\hline $\mathrm{C}(48)-\mathrm{C}(47)-\mathrm{C}(53)-\mathrm{N}(4)$ & $52.7(4)$ \\
\hline $\mathrm{C}(52)-\mathrm{C}(47)-\mathrm{C}(53)-\mathrm{N}(4)$ & $-133.8(3)$ \\
\hline$C(48)-C(47)-C(53)-C(54)$ & $-120.4(3)$ \\
\hline$C(52)-C(47)-C(53)-C(54)$ & $53.0(4)$ \\
\hline$N(4)-C(53)-C(54)-C(55)$ & $-1.7(3)$ \\
\hline$C(47)-C(53)-C(54)-C(55)$ & $172.2(3)$ \\
\hline$C(53)-C(54)-C(55)-N(3)$ & $0.0(3)$ \\
\hline$C(53)-C(54)-C(55)-C(56)$ & $179.4(3)$ \\
\hline$C(62)-C(57)-C(58)-C(59)$ & $0.6(5)$ \\
\hline$C(63)-C(57)-C(58)-C(59)$ & $179.4(3)$ \\
\hline$C(57)-C(58)-C(59)-C(60)$ & $0.2(5)$ \\
\hline$C(58)-C(59)-C(60)-C(61)$ & $-0.3(5)$ \\
\hline$C(59)-C(60)-C(61)-C(62)$ & $-0.5(6)$ \\
\hline$C(60)-C(61)-C(62)-C(57)$ & $1.4(5)$ \\
\hline$C(58)-C(57)-C(62)-C(61)$ & $-1.4(5)$ \\
\hline$C(63)-C(57)-C(62)-C(61)$ & $179.8(3)$ \\
\hline $\mathrm{C}(58)-\mathrm{C}(57)-\mathrm{C}(63)-\mathrm{N}(6)$ & $18.6(4)$ \\
\hline$C(62)-C(57)-C(63)-N(6)$ & $-162.7(3)$ \\
\hline$C(58)-C(57)-C(63)-C(64)$ & $-161 \cdot 3(3)$ \\
\hline$C(62)-C(57)-C(63)-C(64)$ & $17.4(5)$ \\
\hline$N(6)-C(63)-C(64)-C(65)$ & $-0.8(3)$ \\
\hline$C(57)-C(63)-C(64)-C(65)$ & $179.1(3)$ \\
\hline $\mathrm{C}(63)-\mathrm{C}(64)-\mathrm{C}(65)-\mathrm{N}(5)$ & $1.5(3)$ \\
\hline$C(63)-C(64)-C(65)-C(66)$ & $-174.9(3)$ \\
\hline $\mathrm{C}(44)-\mathrm{C}(43)-\mathrm{N}(1)-\mathrm{N}(2)$ & $-2.3(3)$ \\
\hline $\mathrm{C}(37)-\mathrm{C}(43)-\mathrm{N}(1)-\mathrm{N}(2)$ & $173.0(2)$ \\
\hline $\mathrm{C}(44)-\mathrm{C}(43)-\mathrm{N}(1)-\mathrm{Rh}(1)$ & $-176.47(19)$ \\
\hline $\mathrm{C}(37)-\mathrm{C}(43)-\mathrm{N}(1)-\mathrm{Rh}(1)$ & $-1.2(4)$ \\
\hline $\mathrm{C}(44)-\mathrm{C}(45)-\mathrm{N}(2)-\mathrm{N}(1)$ & $0.6(3)$ \\
\hline $\mathrm{C}(46)-\mathrm{C}(45)-\mathrm{N}(2)-\mathrm{N}(1)$ & $-178.5(3)$ \\
\hline $\mathrm{C}(44)-\mathrm{C}(45)-\mathrm{N}(2)-\mathrm{B}(1)$ & $159.9(3)$ \\
\hline $\mathrm{C}(46)-\mathrm{C}(45)-\mathrm{N}(2)-\mathrm{B}(1)$ & $-19.2(4)$ \\
\hline $\mathrm{C}(43)-\mathrm{N}(1)-\mathrm{N}(2)-\mathrm{C}(45)$ & $1.1(3)$ \\
\hline $\mathrm{Rh}(1)-\mathrm{N}(1)-\mathrm{N}(2)-\mathrm{C}(45)$ & $176.49(17)$ \\
\hline $\mathrm{C}(43)-\mathrm{N}(1)-\mathrm{N}(2)-\mathrm{B}(1)$ & $-159.6(2)$ \\
\hline $\mathrm{Rh}(1)-\mathrm{N}(1)-\mathrm{N}(2)-\mathrm{B}(1)$ & $15.8(3)$ \\
\hline $\mathrm{N}(3)-\mathrm{B}(1)-\mathrm{N}(2)-\mathrm{C}(45)$ & $-113.6(3)$ \\
\hline $\mathrm{N}(5)-\mathrm{B}(1)-\mathrm{N}(2)-\mathrm{C}(45)$ & $120.6(3)$ \\
\hline
\end{tabular}




\begin{tabular}{|c|c|}
\hline $\mathrm{N}(3)-\mathrm{B}(1)-\mathrm{N}(2)-\mathrm{N}(1)$ & $43.3(3)$ \\
\hline $\mathrm{N}(5)-\mathrm{B}(1)-\mathrm{N}(2)-\mathrm{N}(1)$ & $-82.5(3)$ \\
\hline $\mathrm{C}(54)-\mathrm{C}(55)-\mathrm{N}(3)-\mathrm{N}(4)$ & $1.6(3)$ \\
\hline $\mathrm{C}(56)-\mathrm{C}(55)-\mathrm{N}(3)-\mathrm{N}(4)$ & $-177.9(3)$ \\
\hline $\mathrm{C}(54)-\mathrm{C}(55)-\mathrm{N}(3)-\mathrm{B}(1)$ & $-164.5(3)$ \\
\hline $\mathrm{C}(56)-\mathrm{C}(55)-\mathrm{N}(3)-\mathrm{B}(1)$ & $16.0(5)$ \\
\hline$N(5)-B(1)-N(3)-C(55)$ & $-101 \cdot 3(3)$ \\
\hline$N(2)-B(1)-N(3)-C(55)$ & $129.9(3)$ \\
\hline $\mathrm{N}(5)-\mathrm{B}(1)-\mathrm{N}(3)-\mathrm{N}(4)$ & $94.3(3)$ \\
\hline $\mathrm{N}(2)-\mathrm{B}(1)-\mathrm{N}(3)-\mathrm{N}(4)$ & $-34.5(3)$ \\
\hline $\mathrm{C}(54)-\mathrm{C}(53)-\mathrm{N}(4)-\mathrm{N}(3)$ & $2.6(3)$ \\
\hline $\mathrm{C}(47)-\mathrm{C}(53)-\mathrm{N}(4)-\mathrm{N}(3)$ & $-171.5(2)$ \\
\hline $\mathrm{C}(54)-\mathrm{C}(53)-\mathrm{N}(4)-\mathrm{Rh}(1)$ & $-157.6(2)$ \\
\hline $\mathrm{C}(47)-\mathrm{C}(53)-\mathrm{N}(4)-\mathrm{Rh}(1)$ & $28.2(4)$ \\
\hline$C(55)-N(3)-N(4)-C(53)$ & $-2 \cdot 6(3)$ \\
\hline$B(1)-N(3)-N(4)-C(53)$ & $164.2(2)$ \\
\hline $\mathrm{C}(55)-\mathrm{N}(3)-\mathrm{N}(4)-\mathrm{Rh}(1)$ & $163.53(18)$ \\
\hline $\mathrm{B}(1)-\mathrm{N}(3)-\mathrm{N}(4)-\mathrm{Rh}(1)$ & $-29.7(3)$ \\
\hline$C(64)-C(65)-N(5)-N(6)$ & $-1.7(3)$ \\
\hline $\mathrm{C}(66)-\mathrm{C}(65)-\mathrm{N}(5)-\mathrm{N}(6)$ & $174.9(3)$ \\
\hline $\mathrm{C}(64)-\mathrm{C}(65)-\mathrm{N}(5)-\mathrm{B}(1)$ & $-159.8(3)$ \\
\hline $\mathrm{C}(66)-\mathrm{C}(65)-\mathrm{N}(5)-\mathrm{B}(1)$ & $16.9(4)$ \\
\hline $\mathrm{N}(3)-\mathrm{B}(1)-\mathrm{N}(5)-\mathrm{C}(65)$ & $169.1(2)$ \\
\hline$N(2)-B(1)-N(5)-C(65)$ & $-66.2(4)$ \\
\hline $\mathrm{N}(3)-\mathrm{B}(1)-\mathrm{N}(5)-\mathrm{N}(6)$ & $12.7(3)$ \\
\hline $\mathrm{N}(2)-\mathrm{B}(1)-\mathrm{N}(5)-\mathrm{N}(6)$ & $137.3(2)$ \\
\hline $\mathrm{C}(64)-\mathrm{C}(63)-\mathrm{N}(6)-\mathrm{N}(5)$ & $-0.2(3)$ \\
\hline $\mathrm{C}(57)-\mathrm{C}(63)-\mathrm{N}(6)-\mathrm{N}(5)$ & $179.9(2)$ \\
\hline$C(65)-N(5)-N(6)-C(63)$ & $1.2(3)$ \\
\hline $\mathrm{B}(1)-\mathrm{N}(5)-\mathrm{N}(6)-\mathrm{C}(63)$ & $161.3(2)$ \\
\hline$C(2)-C(1)-P(1)-C(7)$ & $165.4(2)$ \\
\hline$C(6)-C(1)-P(1)-C(7)$ & $-19.7(3)$ \\
\hline$C(2)-C(1)-P(1)-C(13)$ & $-89.5(2)$ \\
\hline$C(6)-C(1)-P(1)-C(13)$ & $85.4(2)$ \\
\hline $\mathrm{C}(2)-\mathrm{C}(1)-\mathrm{P}(1)-\mathrm{Rh}(1)$ & $40.6(2)$ \\
\hline$C(6)-C(1)-P(1)-R h(1)$ & $-144.5(2)$ \\
\hline$C(12)-C(7)-P(1)-C(1)$ & $85.8(2)$ \\
\hline$C(8)-C(7)-P(1)-C(1)$ & $-90.9(2)$ \\
\hline$C(12)-C(7)-P(1)-C(13)$ & $-16.2(3)$ \\
\hline$C(8)-C(7)-P(1)-C(13)$ & $167.1(2)$ \\
\hline $\mathrm{C}(12)-\mathrm{C}(7)-\mathrm{P}(1)-\mathrm{Rh}(1)$ & $-147.7(2)$ \\
\hline $\mathrm{C}(8)-\mathrm{C}(7)-\mathrm{P}(1)-\mathrm{Rh}(1)$ & $35.6(2)$ \\
\hline$C(14)-C(13)-P(1)-C(1)$ & $33.1(2)$ \\
\hline$C(18)-C(13)-P(1)-C(1)$ & $-151 \cdot 6(2)$ \\
\hline$C(14)-C(13)-P(1)-C(7)$ & $137.0(2)$ \\
\hline$C(18)-C(13)-P(1)-C(7)$ & $-47.7(2)$ \\
\hline $\mathrm{C}(14)-\mathrm{C}(13)-\mathrm{P}(1)-\mathrm{Rh}(1)$ & $-95.0(2)$ \\
\hline $\mathrm{C}(18)-\mathrm{C}(13)-\mathrm{P}(1)-\mathrm{Rh}(1)$ & $80.4(2)$ \\
\hline$C(32)-C(31)-P(2)-C(19)$ & $-127.2(2)$ \\
\hline$C(36)-C(31)-P(2)-C(19)$ & $54.5(2)$ \\
\hline$C(32)-C(31)-P(2)-C(25)$ & $-20.6(3)$ \\
\hline$C(36)-C(31)-P(2)-C(25)$ & $161.1(2)$ \\
\hline $\mathrm{C}(32)-\mathrm{C}(31)-\mathrm{P}(2)-\mathrm{Rh}(1)$ & $108.3(2)$ \\
\hline$C(36)-C(31)-P(2)-R h(1)$ & $-70.0(2)$ \\
\hline$C(20)-C(19)-P(2)-C(31)$ & $-127.6(2)$ \\
\hline$C(24)-C(19)-P(2)-C(31)$ & $50.0(2)$ \\
\hline$C(20)-C(19)-P(2)-C(25)$ & $128.0(2)$ \\
\hline
\end{tabular}




$$
\begin{aligned}
& \mathrm{C}(24)-\mathrm{C}(19)-\mathrm{P}(2)-\mathrm{C}(25) \\
& \mathrm{C}(20)-\mathrm{C}(19)-\mathrm{P}(2)-\mathrm{Rh}(1) \\
& \mathrm{C}(24)-\mathrm{C}(19)-\mathrm{P}(2)-\mathrm{Rh}(1) \\
& \mathrm{C}(30)-\mathrm{C}(25)-\mathrm{P}(2)-\mathrm{C}(31) \\
& \mathrm{C}(26)-\mathrm{C}(25)-\mathrm{P}(2)-\mathrm{C}(31) \\
& \mathrm{C}(30)-\mathrm{C}(25)-\mathrm{P}(2)-\mathrm{C}(19) \\
& \mathrm{C}(26)-\mathrm{C}(25)-\mathrm{P}(2)-\mathrm{C}(19) \\
& \mathrm{C}(30)-\mathrm{C}(25)-\mathrm{P}(2)-\mathrm{Rh}(1) \\
& \mathrm{C}(26)-\mathrm{C}(25)-\mathrm{P}(2)-\mathrm{Rh}(1) \\
& \mathrm{C}(43)-\mathrm{N}(1)-\mathrm{Rh}(1)-\mathrm{N}(4) \\
& \mathrm{N}(2)-\mathrm{N}(1)-\mathrm{Rh}(1)-\mathrm{N}(4) \\
& \mathrm{C}(43)-\mathrm{N}(1)-\mathrm{Rh}(1)-\mathrm{P}(2) \\
& \mathrm{N}(2)-\mathrm{N}(1)-\mathrm{Rh}(1)-\mathrm{P}(2) \\
& \mathrm{C}(43)-\mathrm{N}(1)-\mathrm{Rh}(1)-\mathrm{P}(1) \\
& \mathrm{N}(2)-\mathrm{N}(1)-\mathrm{Rh}(1)-\mathrm{P}(1) \\
& \mathrm{C}(53)-\mathrm{N}(4)-\mathrm{Rh}(1)-\mathrm{N}(1) \\
& \mathrm{N}(3)-\mathrm{N}(4)-\mathrm{Rh}(1)-\mathrm{N}(1) \\
& \mathrm{C}(53)-\mathrm{N}(4)-\mathrm{Rh}(1)-\mathrm{P}(2) \\
& \mathrm{N}(3)-\mathrm{N}(4)-\mathrm{Rh}(1)-\mathrm{P}(2) \\
& \mathrm{C}(53)-\mathrm{N}(4)-\mathrm{Rh}(1)-\mathrm{P}(1) \\
& \mathrm{N}(3)-\mathrm{N}(4)-\mathrm{Rh}(1)-\mathrm{P}(1) \\
& \mathrm{C}(31)-\mathrm{P}(2)-\mathrm{Rh}(1)-\mathrm{N}(1) \\
& \mathrm{C}(19)-\mathrm{P}(2)-\mathrm{Rh}(1)-\mathrm{N}(1) \\
& \mathrm{C}(25)-\mathrm{P}(2)-\mathrm{Rh}(1)-\mathrm{N}(1) \\
& \mathrm{C}(31)-\mathrm{P}(2)-\mathrm{Rh}(1)-\mathrm{N}(4) \\
& \mathrm{C}(19)-\mathrm{P}(2)-\mathrm{Rh}(1)-\mathrm{N}(4) \\
& \mathrm{C}(25)-\mathrm{P}(2)-\mathrm{Rh}(1)-\mathrm{N}(4) \\
& \mathrm{C}(31)-\mathrm{P}(2)-\mathrm{Rh}(1)-\mathrm{P}(1) \\
& \mathrm{C}(19)-\mathrm{P}(2)-\mathrm{Rh}(1)-\mathrm{P}(1) \\
& \mathrm{C}(25)-\mathrm{P}(2)-\mathrm{Rh}(1)-\mathrm{P}(1) \\
& \mathrm{C}(1)-\mathrm{P}(1)-\mathrm{Rh}(1)-\mathrm{N}(1) \\
& \mathrm{C}(7)-\mathrm{P}(1)-\mathrm{Rh}(1)-\mathrm{N}(1) \\
& \mathrm{C}(13)-\mathrm{P}(1)-\mathrm{Rh}(1)-\mathrm{N}(1) \\
& \mathrm{C}(1)-\mathrm{P}(1)-\mathrm{Rh}(1)-\mathrm{N}(4) \\
& \mathrm{C}(7)-\mathrm{P}(1)-\mathrm{Rh}(1)-\mathrm{N}(4) \\
& \mathrm{C}(13)-\mathrm{P}(1)-\mathrm{Rh}(1)-\mathrm{N}(4) \\
& \mathrm{C}(1)-\mathrm{P}(1)-\mathrm{Rh}(1)-\mathrm{P}(2) \\
& \mathrm{C}(7)-\mathrm{P}(1)-\mathrm{Rh}(1)-\mathrm{P}(2) \\
& \mathrm{C}(13)-\mathrm{P}(1)-\mathrm{Rh}(1)-\mathrm{P}(2)
\end{aligned}
$$

$$
\begin{gathered}
-54.4(2) \\
-7.0(2) \\
170.58(18) \\
91.2(2) \\
-88.8(3) \\
-168.3(2) \\
11.7(3) \\
-33.8(2) \\
146.2(2) \\
113.3(3) \\
-60.41(17) \\
-73.9(2) \\
112.42(16) \\
92.7(6) \\
-81.0(5) \\
-134.1(3) \\
66.49(16) \\
-169.8(2) \\
30.8(4) \\
43.5(3) \\
-115.90(16) \\
4.18(11) \\
-107.68(10) \\
123.78(11) \\
38.9(3) \\
-72.9(3) \\
158.6(3) \\
-174.25(9) \\
73.90(9) \\
-54.64(10) \\
31.8(5) \\
-86.2(5) \\
151.2(5) \\
11.54(11) \\
-106.41(11) \\
130.91(12) \\
-161.71(10) \\
80.35(10) \\
-42.33(11) \\
\end{gathered}
$$

